LBL-37342

\title{
QUANTUM SYMMETRY AND PHOTOREACTIVITY OF AZABENZENES
}

\author{
JAMES DAVID MARK CHESKO \\ Ph.D. Thesis \\ DEPARTMENT OF CHEMISTRY \\ University of California, Berkeley \\ and \\ CHEMICAL SCIENCES DIVISION \\ Lawrence Berkeley Laboratory \\ University of California \\ Berkeley, CA 94720
}

JUNE 1995

This work was supported by the Director, Office of Energy Research, Office of Basic Energy Sciences,

Materials Sciences Division, of the U.S. Department of Energy under Contract No. DE-AC03-76SF00098. 


\section{DISCLAIMER}

This report was prepared as an account of work sponsored by an agency of the United States Government. Neither the United States Government nor any agency thereof, nor any of their employees, make any warranty, express or implied, or assumes any legal liability or responsibility for the accuracy, completeness, or usefulness of any information, apparatus, product, or process disclosed, or represents that its use would not infringe privately owned rights. Reference herein to any specific commercial product, process, or service by trade name, trademark, manufacturer, or otherwise does not necessarily constitute or imply its endorsement, recommendation, or favoring by the United States Government or any agency thereof. The views and opinions of authors expressed herein do not necessarily state or reflect those of the United States Government or any agency thereof. 


\section{DISCLAIMER}

Portions of this document may be illegible in electronic image products. Images are produced from the best available original document. 
Quantum Symmetry and Photoreactivity of Azabenzenes

by

James David Mark Chesko

B.Sc. (University of British Columbia) 1987

A dissertation submitted in partial satisfaction of the

requirements for the degree of

Doctor of Philosophy

in

Chemistry

in the

GRADUATE DIVISION

of the

UNIVERSITY of CALIFORNIA at BERKELEY

Committee in charge:

Professor Yuan T. Lee, Chair

Professor C. Bradley Moore

Professor Yuen-Ron Shen

1995 


\title{
Quantum Symmetry and Photoreactivity of Azabenzenes
}

\author{
Copyright @ 1995
}

by

J.D.M. Chesko

The U.S. Department of Energy has the right to use this document for any purpose whatsoever including the right to reproduce all or any part thereof 


\author{
Abstract \\ Quantum Symmetry and Photoreactivity of Azabenzenes \\ by \\ James D.M. Chesko \\ Doctor of Philosophy in Chemistry \\ University of California at Berkeley \\ Professor Yuan T. Lee, Chair
}

The fundamental processes associated with a photochemical reaction are described with reference to experimentally determined properties of azabenzenes. Consideration of both excitation and relaxation processes led to the presentation of the symmetry propagator, a unifying principle which maps system fluctuations (perturbations acting on an initial state) with dissipations (transitions to different states), thus directing the energy flow along competing reactive and non-reactive pathways. A coherent picture of relaxation processes including chemical reactions was constructed with the aid of spectroscopic data.

Pyrazine ( 1,4 diazine) possesses vibronically active modes which provide an extremely efficient mechanism for internal conversion to the first excited singlet state, where other promoting modes of the correct symmetry induce both intersystem crossing to the triplet manifold, isomerization through diaza-benzvalene, and chemical reactions through a $\left[\sigma_{s}{ }_{s}+{ }_{\sigma} 2_{s}\right]$ cycloreversion of dewar pyrazine to yield $\mathrm{HCN}$ plus an azete. At higher energies simple $\mathrm{H}$ atom loss and internal conversion become more predominant, 
leading to ring opening followed by elimination of methylene nitrile and thermal (ground state) reaction products.

The efficiency of chemical transformations as dissipation mechanisms versus competing fluorescence, phosphorescence and radiationless relaxation was mapped from the near ultraviolet $\left(S_{1}\right.$ excitation at $\left.308 \mathrm{~nm}\right)$ to the far ultraviolet ( 248 and $193 \mathrm{~nm}$ excitation to $S_{2}$ and $S_{3}$ ) by photodissociation quantum yields into reaction channels characterized by molecular beam photofragment translational spectroscopy. Based upon orbital symmetry correlations and ab initio calculations a reaction path model for azabenzene photochemistry was presented and tested against experimental results. The presence of undiscovered channels in other azabenzene systems was predicted and then experimentally verified. The dominant process, $\mathrm{HCN}$ elimination, was resolved into three distinct channels representing different paths or co-products. Both molecular and atomic hydrogen elimination was observed, the former possessing significant vibrational excitation. Small yields of isomerization products, acetylene and molecular nitrogen, were also observed. 


\section{Table of Contents}

Chapter I. Azabenzene Photochemistry

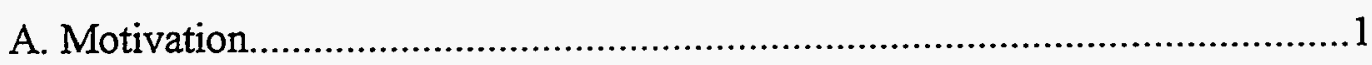

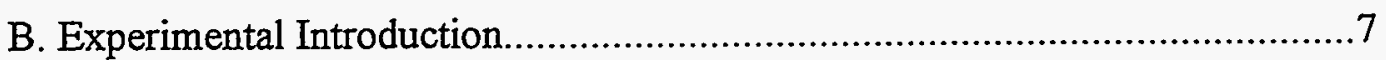

1. Translation Energy Distributions Peaked near zero........................8

2. Measurement of Absolute Quantum Yields.................................13

C. Experimental Data

1. Pyrazine Photodissociation following 308nm excitation................. 17

2. Pyrazine Photodissociation following $248 \mathrm{~nm}$ excitation.................25

3. Pyrazine Photodissociation following $193 \mathrm{~nm}$ excitation...................36

4. 1,3,5 Triazine Photodissociation following $248 \mathrm{~nm}$ excitation...........39

5. Ab Initio studies of Valence isomers and Azetes........................41

6. Map of Azabenzene Photoreactivity........................................47

7. An Application: Reinterpreting the Phenomenon of Supercollisions.............................................................48

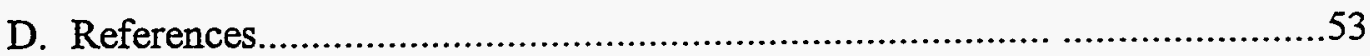

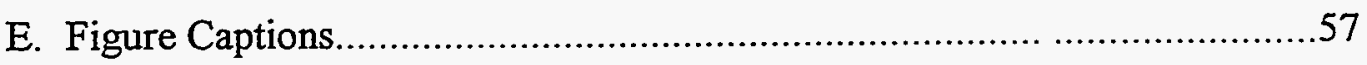

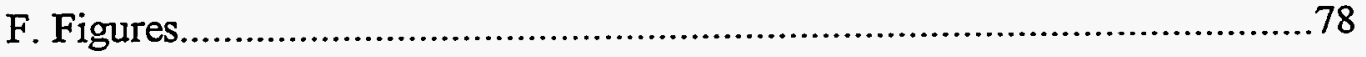


Chapter II. Discussion

A. Symmetry, Spectroscopy and Dynamics..........................................149

B. Orbitals, Symmetry and Photoreactivity.......................................... 158

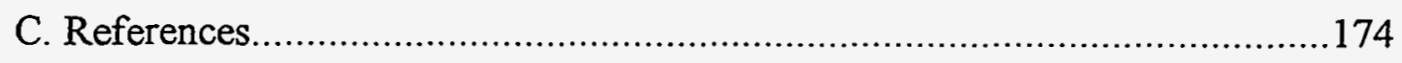

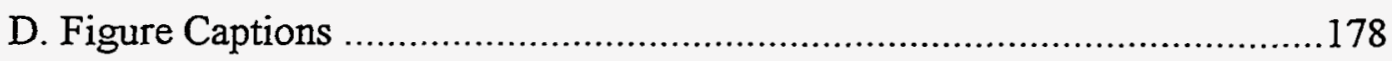

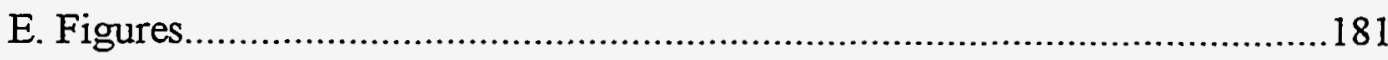

\section{List of Tables}

Chapter I.

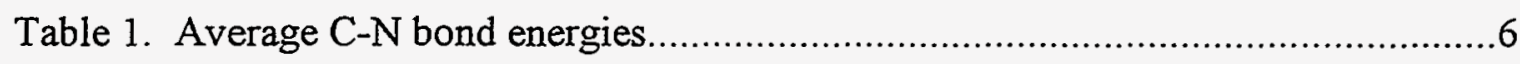

Table 2. Electron Impact spectra of selected azabenzenes ..................................

Table 3. Absolute Quantum Yields of Pyrazine Photodissociation..............................15

Table 4. Photodissociation Products of Pyrazine following 308nm excitation..............25

Table 5. Photodissociation Products of Pyrazine following 248nm excitation...............26

Table 6. Photodissociation Products of Pyrazine following $193 \mathrm{~nm}$ excitation................37

Table 7. Photodissociation Products of s-Triazine following 248nm excitation.............39

Table 8. Summary of $\mathrm{Ab}$ initio results......................................................43

Chapter II.

Table 1. Franck-Condon Factors (vibrational overlap).................................... 154

Table 2. Important Mechanisms of Energy Transfer......................................157 


\section{Acknowledgements}

Chaos and Heisenberg aside, it is with great uncertainty that I look both forward from and backwards at my sojourn in graduate school. The challenging intellectual atmosphere, unrelenting laser difficulties and sympathy of family, friends and co-workers slowly transformed my idealism into a more realistic view of the world around me. The opportunity to work with many great minds science was certainly a privilege and a learning experience.

To the reader who may continue to browse these pages, I first offer an apology. The magnum opus which was intended to read like the poetry of T.S. Eliot resembles more a Henry Fielding novel without the humor. Perhaps some recompense can be found in the conceptual leap which attempted to show how symmetry derived properties can manifest themselves in a variety of important ways, from spectroscopy to chemical reactivity. For those questioning how obscure and irrelevant a thesis thus titled can possibly be, consider for a moment that the primary mechanism of photo-induced DNA damage involves the formation of cyclobutadiene pyrimidine dimers. Experimenters have observed efficient triplet energy transfer in this reaction and the requirement of light in the repair mechanism by DNA photolyase. The role of photoexcited states, triplet hypersurfaces and cycloaddition may be integral processes in the proofreading of the genetic code and therefore your existence.

Through the process of writing it has become clearer to me how much a cooperative effort research has been. From the long, challenging hours (years!) shared on the picosecond project I was shown guidance, resolve and skills that I would need to 
continue in later years. Jim Myers was a mentor that was willing and able to respond to the numerous challenges we faced while keeping a sharp mind open to fascinating areas such as coherent control, chaos and the numerous holy grails we searched for during the picoyears. That the pages of this thesis do not contain the final chapter of the picoproject is a reflection of the inverse theorem-- the many years and books chronicling our technical struggle have left little of scientific import. The perspective that Matt Côté gave to the project-- his appreciation of quantum mechanical paradoxes, his positive attitude in the face of experimental difficulties and fishing stories helped bolster morale on a daily basis.

The latter years proved the value of teamwork and support in the lab. Prof. Lee's story of working by himself when Dr. Mahan went on sabbatical was a prelude to the situation our lab found itself in when Prof. Lee retired and accepted a prestigous post in Taiwan. I greatly value his scientific ability and hope he will share his insights on the contents of this thesis one day. Through the last few months the experiments presented in this thesis were performed because of the determination and support of labmates who understood our common lot. Dr. Miau (T-T) provided invaluable assistance in watching over the machine while I grabbed Z's even despite an impending filing deadline which he satisfied. The trips to Chinatown, lessons in cooking, computers and correlation functions only shadow his many talents. I hope one day we will be able to walk the streets of Taipei, shop for bananas and speak Mandarin without getting too many puzzled looks.

The consideration and support of Laura Smoliar and Cheryl Longfellow has certainly made the last few hectic months easier to bear. Their feedback has brought me back to conditions much closer to a 'normal operating range' rather than a 'self-destruct 
sequence' for I do not distinguish easily between the two. The parties, dinners and conversation have reminded us of a social dimension to our lives, as well as to greater balance and less stress.

The individuals which have provided discussion and differing perspectives have made the Lee lab a truly unique place to work. Dr. Stranges showed me all I needed to know to run the rotating source machine as well as providing great Italian dinners and company to watch the World Cup; Dr. Boo (Doo Wan) has tried to enlighten me with his Buddhist philosophy and we have shared time and meditation on many thoughts; Hongtao has been an expert reference on molecular beams and was a great partner to work with briefly on the lithium photoexcitation project; Vlad answered all the questions I needed to know about the Soviet Union and helped me with my Russian (which came in handy translating some $a b$ initio work cited herein); Ralf has been a staunch supporter of correlation diagrams and a knowledgeable person to discuss interstellar chemistry with.

Without the encouragement and support which my parents and family have provided I certainly would not have made it this far. Their respect for individual choice, value of education and regular visits have given me an opportunity which was not readily available to them or their parents. Despite the enormous difficulties and disappointments that this opportunity has carried with it, it is a step towards the top of a mountain which will take a lifetime to reach. In my time I have been fortunate to find someone who will share in the dreams which have been a large part of my graduate school efforts, and which we now will pursue together. 


\section{Azabenzene Photochemistry}

\section{A. Motivation}

Years of working on an experimental photochemical project led me to consider what qualitative differences in reactivity could arise from details of the initial excitation process. Discussions with postdoctoral colleagues impressed upon me how much specific information could be determined from spectroscopic studies, yet the role of chemical dynamics in such investigations was often disguised in broadened linewidths and (presumed) dissociating states. The case of diazine chemistry stood out as a notable challenge: its spectroscopic parameters were well characterized, yet its photochemistry was still unknown. Additional motivation for exploring this system, to search for bridges and connections between the often uncommunicative disciplines of spectroscopy, synthetic chemistry and gas phase reaction dynamics, came from years of mostly watching and seeing how each field carved out its own channel, vocabulary and method of inquiry.

In broaching the subject of thermal versus photo-reactions with my supervisor I was kindly reminded of the principle which states how internal conversion will rapidly bring the most highly excited state to the ground state surface, where barrier heights and transition state geometry will dictate the chemical reaction paths. In processes such as combustion thermokinetics and the role of radicals as intermediates in driving addition and fragmentation processes are important. However, in processes involving photoexcitation this need not be necessarily so. The 'Woodward and Hoffman' rules of 
reaction symmetry predict opposite behavior for '(photo-)excited states' compared to ground state reactions, and years of study of biochemical systems had instilled an appreciation of specificity and 'purpose' in chemical transformations. The lowest lying excited states, of $\mathrm{n} \pi$ character, had relatively weak oscillator strengths and relatively long lifetimes. The possibility of experimental describing reactive selectivity in the unbiased framework of physical laws and principles was a notable challenge.

Many simple aromatic systems, cyclic compounds, and azabenzenes had been studied before, both in our lab and in other labs. On one extreme was the compound benzene, $\mathrm{C}_{6} \mathrm{H}_{6}$, whose reactive behavior from earlier studies had predicted primarily $\mathrm{H}$ and $\mathrm{H}_{2}$ elimination with little loss of integrity in the conjugated ring system. On the other extreme were triazine and tetrazine, which were thought to undergo rapid, concerted triple dissociation. In the middle were the diazabenzenes, which had been subject to extensive spectroscopic investigation as a model system for electronic relaxation pathways, but whose reactive behavior was scarcely known. A single reference to triple dissociation following excitation at wavelengths less than $160 \mathrm{~nm}$ along with the capability to isomerize were the starting points of my investigation of photodynamics. The role of valence isomer intermediates could possibly be studied due to the two nitrogens which would 'define' positions within the ring in a manner that unsubstituted benzene and pyridine could not. Also, the nitrogen heteroatoms would destabilize the reactant with respect to benzene by about an electron volt, and also stabilize the products about one electron volt per nitrogen, tipping the scale in favor of producing products whose precursors could be investigated. The reduced symmetry of 
the system $\left(D_{2 h}\right.$ vs. $\left.D_{6 h}\right)$ would remove some of the degeneracies and facilitate different transitions which will be discussed in greater detail later.

The spectroscopic properties of 1,4 Diazine were similar to other azabenzenes. The dipole transitions are usually discussed with reference to similar transitions in benzene, with the additional excitations possible from the non-bonding electrons on the nitrogen. The transition energies provide a rough estimate of the $\beta$ value associated with orbital stabilization due to delocalization of the $\pi$-bonded network whose molecular orbitals can be described from linear combination of atomic orbital wavefunctions:

$$
\beta=\int \phi_{1} H \phi_{2} d \tau
$$

where $\beta$ is often referred to as the vertical resonance integral (see ref. 1). By vertical, we mean that the internuclear distances constrained by symmetry ( $\mathrm{D}_{\not h}$ for pyrazine) are held fixed in the nonresonating (hypothetical) molecule and the energy difference calculated by descending vertically into the resonating form. The experimentally measured stabilization energy will thus be a sum of this term $\left(R_{v}\right)$ and the energy associated with stretching and compressing the bonds to achieve the desired structural geometry $\left(E_{c}\right)$ :

$$
R_{\text {exp }}=R_{v}+E_{c}
$$


For benzene estimates of about $37 \mathrm{kcal} / \mathrm{mol}$ for $E_{c}$ lead to a $\beta$ of roughly $-37 \mathrm{kcal}$ since the overall stabilization for the benzene molecular orbital configuration is $2|\beta|$ (see ref. 2). For pyrazine we would expect a similar but smaller value for $E_{c}$ since we have already 'broken' $D_{6 h}$ symmetry and are thus equilibrating four bond lengths rather than six. The vertical resonance energy is expected to be smaller as well, since the electronegativity difference between the subsituted nitrogens relative to carbon will result in greater localization around the nitrogen centers.

If the Huckel approximation of negligible overlap is not used in our treatment in hopes of obtaining a more rigorous understanding of $\beta$ comes from solving the defining secular equation:

$$
\left|\begin{array}{cc}
\left(H_{11}-E\right) & \left(H_{12}-E S_{12}\right) \\
\left(H_{21}-E S_{21}\right) & \left(H_{22}-E\right)
\end{array}\right|=0
$$

where the $S_{i j}$ allow for non-negligible overlap between neighboring $p \pi$ orbitals, $E$ is the true zero of energy of an electron removed from the molecule and taken hypothetically to infinite separation, so that $\beta=\mathrm{H}_{12}-\mathrm{ES}_{12}$ (ref. 3 ). The value of $\mathrm{E}$ is thought to be on the order of $50 \mathrm{eV}$ and $\mathrm{S}$ around 0.2 , so that the $\beta$ is a difference between two large numbers and is about $1-2 \mathrm{eV}$. The value $\beta$ for benzene, diphenyl, napthalene, anthracene and phenanthrene are all empirically found to lie in the -18 to -21 
$\mathrm{kcal} / \mathrm{mol}$ range. To fit spectra of benzene and unsaturated hydrocarbons within the Huckel approximation large $\beta$ values on the order of $55-60 \mathrm{kcal} / \mathrm{mol}$ are needed, and these values are often referred to as the 'spectroscopic value for $\beta$.'

The increase in product stability is most closely related to the energy obtained by localizing an electron on one of the atoms, as we would imagine in the case of an isolated atom, and is referred to as $\alpha$ :

$\alpha=\int \phi_{1} \mathrm{H} \phi_{1} \mathrm{~d} \tau$

As we substitute the more electronegative nitrogen for the carbon, the $\alpha$ value is expected to lower as the heat of formation of $\mathrm{HCN}$ ( $32.3 \mathrm{kcal}$, ref. 4) relative to $\mathrm{C}_{2} \mathrm{H}_{2}$ (54.2 kcal, ref. 4) demonstrates. The fact that the heat of formation of pyrazine is higher than benzene by about $26 \mathrm{kcal}(\mathrm{leV})$ is a consequence of the reduced resonance stabilization $|\beta|$. This is exemplified by the series benzene $(20 \mathrm{kcal}) \rightarrow$ pyridine (34 $\mathrm{kcal}) \rightarrow$ pyrazine $(46 \mathrm{kcal}) \rightarrow$ triazine $(54 \mathrm{kcal})$, with the heats of formation taken from references 4 and 34 . The driving force to aromaticity and thus internal conversion to 'ground state' chemistry is weakened by this substitution, as is markedly observed in the photochemistry of triazine and tetrazine versus benzene. The former compounds are thought to synchronously decompose by triple dissociation into three fragments, while the latter does not yield any analogous products. The electron density of the highest occupied molecular orbital (HOMO) as determined by ab initio methods shows the 
tendency towards localization that is reflected in the chemical shift of the protons as recorded by nuclear magnetic resonance. The 'inductive' effect of the nitrogens draws electron density away from the carbons, thus deshielding the hydrogens bonded to them.

The comparison of the $1, n$ diazines show pyrazine to be somewhat balanced by the symmetric positions of the nitrogens. Shifting the second nitrogen from the para (4) position to the meta (3) position is an isoenergetic process, however continuing to the ortho (2) position is energetically unfavorable by about $20 \mathrm{kcal}(1 \mathrm{eV})$. By comparing average bond strengths between carbon and nitrogen atoms (see table 1) we may gain insight into the plausibility and importance of various valence isomers based upon connectivity alone (what protein chemists would refer to as the primary structure).

Table 1. Average Bond Energies from ref. 5.

\begin{tabular}{c|rrrrrr}
\hline & Single & Bond & Double & Bond & Triple & Bond \\
\hline Atom $1 \downarrow / 2 \rightarrow$ & $\mathrm{C}$ & $\mathrm{N}$ & $\mathrm{C}$ & $\mathrm{N}$ & $\mathrm{C}$ & $\mathrm{N}$ \\
$\mathrm{C}$ & 83 & 73 & 146 & 147 & 200 & 213 \\
$\mathrm{~N}$ & & 39 & & $\mathrm{c} .150$ & & 220 \\
\hline
\end{tabular}

Structural isomerization within a carbon-nitrogen sigma bonded framework is expected to be rather isoenergetic due to the comparable bond strengths, with the notable exception of the formation of nitrogen-nitrogen single bonds, which are energetically disfavorable on the order of $2 \mathrm{eV}$. The transition from a delocalized pi network to a more localized, saturated bonding arrangement would be promoted by the 
electronegativity difference of the heteroatom (nitrogen) which also provide two lone pairs of electrons which can interact both through bond as well as contributing to resonance structures and important expanded valence intermediates. Describing the bonding topology of the conjugated system in transit along reaction coordinates to products will serve as a systematic method of describing the reaction path with reference to orbital correlations and interactions among the excited state surfaces.

\section{B. Experimental Introduction}

Details of the photodissocation process were studied by photofragment translational spectroscopy. The experimental apparatus consists of a rotatable molecular beam source which is crossed orthogonally by a laser beam, with product fragments ionized by electron impact, quadrupole mass filtered and detected by a Daly-type doorknob/photomultiplier and has been described in detail earlier (ref. 6). Briefly, a gas mixture of dilute azabenzene (Aldrich Chemical, $98+\%$ ) is seeded in a noble carrier gas to produce a hyperthermal molecular beam when expanded isentropically into a low pressure chamber (see figure 1-1). The beam was skimmed twice along the centerline axis to facilitate differential pumping to lower background gas pressure (and the interference it may cause) and achieve a degree of spatial resolution and uniformity. The pressure gradient of the expanding gas drives the flow work, which converts the enthalpy into increased mean velocity as the gas cools:

$$
V^{2}=2\left(h_{0}-h\right)=2 \int_{T}^{T_{0}} C_{p} \cdot d T
$$


where $\mathrm{V}$ is the mean velocity, $h_{0}$ is the stagnation enthalpy per unit mass (constant along any streamline), $C_{p}$ is the heat capacity (equal to $\gamma /(\gamma+1)(R / W)$, where $\gamma=C_{p} / C_{v}, R$ the universal gas constant and $\mathrm{W}$ is the mean molecular weight.) As the collisional frequency falls off rapidly with decreasing pressure continuum flow is replaced by free molecular flow as the beam approaches the terminal velocity

$$
V_{\infty}=\sqrt{\frac{2 R(\gamma)\left(T_{0}-T\right)}{W(\gamma-1)}}
$$

where the mean velocity of each species is similar, thus allowing us to 'speed up' a heavy molecule by seeding it in a light carrier gas.

The velocity of the beam was found to be accurately described by equation (6) with the assumption $T \ll$ To. The terminal velocity was typically $1720 \mathrm{~m} / \mathrm{s}$ corresponding to $\mathrm{W}=4.15 \mathrm{amu}$ (for a 0.0017 mole fraction of pyrazine seeded in helium). The beam energy resolution was a more complicated function of source pressure and diameter and was proportional to $T / \mathrm{V}^{2}$ (see ref 7). The resolution was described in terms of the speed ratio $v_{0} / \alpha$ which relates the beam intensity to the distribution

$$
N(v)=v^{2} e^{-\frac{\left(v-v_{o}\right)^{2}}{\alpha^{2}}}
$$

and was measured by chopping the beam as well as spatial hole-burning and fit according to the KELVIN program. Because of the modest vapor pressures attainable for the diazine samples the speed ratios measured fell into the 25-45 range. Although the dilute beam meant more time needed to be spent signal averaging, there were some notable benefits which deserve comment. 


\section{Translational Energy Distributions Peaked near zero}

A weakness of molecular beam experiments is the difficulty in measuring product channels which cannot scatter away from the intense background signal. The process of skimming the beam helps reduce the contribution of perpendicular velocity components (angular spread) and a large speed ratio will narrow the spread of parallel components. Since the measured signal is a convolution of the translational energy release due to the reaction and the inherent beam spread the sensitivity to small changes in translational energy increases like the square of the speed ratio (cf. $T / V^{2}$ formula in ref. 7).

The difficulty in measuring signal 'near the beam' is dominated by the background counts. Due to the method of electron impact ionization, substantial fragmentation of the parent will occur and from consultation of a mass spectral atlas one will often find that such fragments match photofragmentation patterns. Electron impact spectrum from the aromatic systems of benzene, pyridine, pyrazine and s-triazine will be discussed (see table 2 below) as a concrete example.

Table 2. Electron Impact spectrum of Benzene and three Azabenzenes (70eV, ref.8)

\begin{tabular}{l|lccc}
\hline mass/charge & Benzene & Pyridine & Pyrazine & s-Triazine* \\
\hline Parent=M & 1.000 & 1.000 & 1.000 & 1.000 \\
M-1 & 0.137 & 0.122 & 0.008 & $<0.002$ \\
M-2 & 0.062 & 0.005 & 0.001 & $<0.005$ \\
M-26 & 0.410 & 0.090 & 0.013 & 0.109
\end{tabular}




\begin{tabular}{l|llll} 
M-27 & 0.302 & 0.705 & 0.391 & 0.719 \\
M-28 & 0.272 & 0.358 & 0.100 & 0.187 \\
M/2 & 0.208 & $0.120(\mathrm{~m} / \mathrm{e} 39)$ & 0.010 & $0.177(\mathrm{~m} / \mathrm{e} 41)$ \\
$M / 2-1$ & 0.065 & $0.064(\mathrm{~m} / \mathrm{e} 38)$ & 0.018 & $0.120(\mathrm{~m} / \mathrm{e} 40)$ \\
27 & 0.062 & 0.067 & 0.023 & 0.509 \\
26 & 0.089 & 0.210 & 0.322 & $<.005$ \\
\hline
\end{tabular}

The background corrected time-of-flight photodissociation spectrum is :

$\mathrm{S}(\mathrm{m} / \mathrm{e}, \mathrm{t}) \propto($ photoreactive product ionized + all fragments $)$

- (parent fragmentation)

$\propto$ (photoexcitation $\times \Sigma$ branching ratios)

$x$ (ionization efficiencyxsurvivability)

$+(1$-photoexcitation $) \times$ parent ionization $\times$ fragmentation

- (1) $\times$ parent ionization $\times$ fragmentation

The most notable property of equation (8) is that it represents a difference signal which is a sum of contributions from potentially numerous pathways, both reactive and nonreactive. For products appearing with little translational energy (i.e. near the carrier beam velocity) this can cause serious complications. The difficulty in measuring signal at $\mathrm{m} / \mathrm{e}=\mathrm{M}-1$ (atomic hydrogen loss) and $\mathrm{M}-27$ ( $\mathrm{HCN}$ loss for the azabenzenes) is evident from the tables. Since the (M-1) peak is about eighty times more massive than the 
recoiling $\mathrm{H}$ atom it will scatter little out of the beam. Assuming an ionization cross section equal to the parent (calculation from additive atomic polarizabilities gives a mere $5 \%$ difference), for a peak ( $>0$ ) to appear on beam axis, the branching ratio into this channel must exceed 0.137 for benzene. A branching ratio less than this will produce a 'hole' (a dip, $\mathrm{S}<0$ ); one roughly equal to this will produce a null. Dramatic experimental verification of such behavior can be visualized in figure 1-2. The difficulty is exacerbated further by realistic consideration of the background (noise) level. For an excitation probability of $1 \%$ that leaves 100 times greater a concentration of parent molecules whose background contribution (and fluctuations) must be subtracted and averaged out effectively. The total molecular beam pulse is typically an order of magnitude longer than the photoexcited portion, magnifying the reactive to unreactive disparity further. The problems inherent in measuring such a signal-- poor signal to noise, an asymmetric profile, an intensity dependence that can deviate from linearity-- have often led researchers to dismiss peaks appearing near the beam velocity as artifacts. The pitfalls of ignoring these signals can be just as difficult, however. In the case of s-triazine numerous 'unseen' channels were observed that escaped previous photodissociation studies. A detailed description of photoreactivity in this system may have been hampered by such an omission, as will be discussed in section 4 .

Through the work on pyrazine several strategies were implemented to overcome these difficulties. Standard methods such as looking for the faster (light) co-fragment were employed. In addition, study of the (electron impact) energy dependent mass spectrum of parent as well as fragments suggested favorable choices. For example, 
although the parent pyrazine ionized and cracked into $53 / 52$ with a $4 / 1$ ratio, the photoproduct 53 produced $53 / 52$ with a $1 / 4$ ratio, thus boosting signal by four and reducing noise by the same factor.

Understanding the mechanisms of ionization, rearrangement and fragmentation within the azabenzene system was necessary to sort out the numerous channels present. For example, the intensities in $\mathrm{m} / \mathrm{e}=26$ for pyrazine and $\mathrm{m} / \mathrm{e}=27$ for triazine increase with increasing electron impact energy and are due to secondary fragmentation of the $m / e=53$ and 54 moieties (whose intensities decreases with electron energy.) Self-consistency in contributions from $\mathrm{m} / \mathrm{e}=54,53,52,51,50,49$ could thus be checked not only by momentum matching, but also by the intensity patterns of $\mathrm{m} / \mathrm{e}=28,27,26,25$ signals.

Figure 1-3 describes the ionization and fragmentation mechanism according to reference 9 , with experimentally measured pathway yields for pyrazine. The role of hydrogen in these transformations was supported by measuring the corresponding deutero-pyrazine (pyrazine- $\mathrm{d}_{4}$ ) mass spectra as well as high resolution mass spectra to determine elemental composition. Additional evidence to guide several channel assignments came from confirming their presence in other azabenzene systems.

A most difficult challenge to address was whether such slow fragments are the result of dissociative ionization. Having absorbed a quanta of light, would the fragmentation pattern be altered as to give rise to the peaks and holes being measured? The most conclusive evidence was the $\mathrm{m} / \mathrm{e}=40$ channel, which was closed following $308 \mathrm{~nm}$ excitation, opened up at $266 \mathrm{~nm}$ and became two distinct peaks through $193 \mathrm{~nm}$. The dramatic shift in branching ratios of these slow channels throughout the energy 
range studied suggested a reaction channel passing over an energetic threshold. The extremely narrow parent velocity distribution afforded meaningful comparison of product signal contours, resolving the significant differences between each band. The ability to create signals which were the superposition of a hole and a peak by varying the excitation probability and thus the relative contribution of each component supported the necessity of using equation (8) to interpret the data (see figure 1-2).

Additionally, the narrow velocity distribution allowed a more sensitive sampling of signal near this region of the time-of-flight. It also provides a very simple explanation for the phenomenon of having every $\mathrm{P}\left(\mathrm{E}_{\mathrm{rrans}}\right)$ peaked slightly away from zero, with the peaking being an artefact of interference between a hole and a true peak. The effect of this interference is enhanced at low angles due to the narrow distribution of parent beam velocities, and decreases as one moves to higher angles off axis of the beam, consistent with the increased contribution of the higher energy tail relative to the unreactive signal. As the product fragments gain enough translational energy to be scattered either ahead of or out of the beam it crosses into the regime where only the first term of equation (8) contributes significantly and the qualitative properties of the spectra change dramatically. There are no longer background contributions from unreactive or unexcited parent and the possibility of the interference effects (peaks, holes and nulls) described above disappear.

\section{Measurement of Absolute Quantum Yields}


The challenge of measuring an absolute quantum yield was motivated by the desire to account for possible radiative as well as reactive relaxation processes. Methods involving calculation of molecular beam density, ionization efficiency, and other parameters are order of magnitude estimates at best. From a hole burning spectrum it was conjectured that a more accurate determination could be made by a method of ratios. Since the primary absolute quantum yield $\Phi_{\text {photodissociation }}$ is simply

$$
\Phi_{\text {photodissociation }}=(\% \text { reacts }) /(\% \text { absorbs a photon })
$$

one could simply integrate over the hole and compare its area to that of the undepleted signal. This would represent the fraction of molecules which react and could be converted to an absolute photodissociation cross-section $\sigma_{\mathrm{pd}}$ if one knew the photoexcitation source intensity and molecular extinction coefficient. Factors such as beam number density, ionization and detection efficiency should cancel out through the division operation.

The above statements are true in the limit of a beam with a delta-function like velocity distribution. For a realistic beam, the temporal separation of photoexcitation, drift, ionization and detection will permit the spatial evolution of the hole as it diffuses outwards (away from its spatial center) and the surrounding beam migrates inwards to fill the hole. Propagating equation (7) over a time interval demonstrates this effect: the velocity distribution remains constant but the spatial extent of the pulse expands linearly as the product of velocity and time. This spreading or stretching factor is easily calculable from knowledge of the initial excitation geometry and beam parameters (fit 
from the KELVIN program.) In practice, a beam profile was taken both by laser holeburning and by running a chopper wheel (located at a different physical position) to confirm the intensity attenuation resulting from spatial spread as a function of beam propagation distance. A narrow velocity distribution (speed ratio c. 30) resulted in a crisp, sharp hole with relatively minor interference due to 'filling', as the uncorrected values approaching unity suggest.

The spatial burn patterns of the laser defined the interaction region, a 5 by $2 \mathrm{~mm}$ cross section with the long axis pointing towards the detector (see figure 1-4). Since the hole burning spectrum was analyzed directly for velocity and speed ratio these numbers could be used directly to characterize the beam. For example, a $1720 \mathrm{~m} / \mathrm{s}$ beam with speed ratio of twenty-seven would intersect a 2.9 microsecond $(5 \mathrm{~mm})$ beam volume which stretched an additional $14 \mathrm{~mm}$ on transit to the ionizer where it was ionized, mass filtered and ultimately detected as a $22 \mathrm{~mm}$ ( 13 microsecond) pulse. From the count rate of the dark (laser off) pulse, 104k/microsecond, the unreacted component is $1352 \mathrm{k}$ while the hole is $96 \mathrm{k}$, implying 0.071 reacted. From the measured laser intensity of 1.7 $\mathrm{mJ} /$ pulse the excitation probability of 0.106 (with absolute cross sections for $248 \mathrm{~nm}$ taken from reference 10) implies a quantum yield of 0.67 . Increasing and decreasing the power by a factor of four changes the value by less than ten percent, providing a reasonable index of the reproducibility of the result. The influence of multiphoton processes on this particular measurement are negligible, since most of the products absorbing a single photon will photodissociate without need of further excitation. The quantum yields at the wavelengths studied are summarized in table 3. 
Table 3. Absolute Quantum Yields of Photoproducts from Pyrazine Photodissociation.

\begin{tabular}{l|ll}
\hline Excitation Energy & Absolute Quantum Yield $\Phi$ & $\sigma_{\text {photodissociation }}\left(\mathrm{cm}^{2}\right)$ \\
\hline$\lambda=308 \mathrm{~nm}$ & $<0.003$ & $<1 \times 10^{-20}$ \\
$\lambda=266 \mathrm{~nm}$ & $0.50 \pm .11$ & $2.5 \pm .5 \times 10^{-18}$ \\
$\lambda=248 \mathrm{~nm}$ & $0.67 \pm .14$ & $3.4 \pm .7 \times 10^{-18}$ \\
$\lambda=193 \mathrm{~nm}$ & $0.90 \pm .20$ & $6.0 \pm 1.3 \times 10^{-18}$ \\
\hline
\end{tabular}

The uncertainty is estimated based upon random errors that factor into the determination of the ratio:

$$
\left(\frac{\Delta f}{f}\right)^{2}=\sum_{i=1}^{n}\left(\frac{\partial f}{\partial x_{i}}\right)^{2}\left(\Delta x_{i}\right)^{2}
$$

where the variables $\mathrm{x}$ are the interaction geometry $(5 \times 2 \mathrm{~mm}+10 \%)$, the absorption cross section $( \pm 5 \%)$, intensity measurement $(10 \%)$ and the integration statistics $( \pm 15 \%)$. The relative trend is consistent with spectroscopic work that suggests a fast radiationless pathway which opens around $34000 \mathrm{~cm}^{-1}$ and dramatically reduces the fluorescence and phosphorescence yields. The suggestion by Yamazaki et al. (ref. 11) that photoisomerization pathways were involved is challenged by measured quantum yields (c. 0.05 , refs. 12-13) for these processes but sustained by the mechanism of photochemical reaction. The particular method just described will not readily recognize 
isomerization processes as reactions, in as far as the ionization processes of the isomer do not differ from the parent pyrazine.

Systematic sources of error may include incomplete irradiation of the volume scattering into the detector, 'hole filling' (mixing diffusion), non-homogeneous laser intensity profiles and absorption contours. All except the last are not expected to exceed the $10-20 \%$ range, based upon the near unity yield at $193 \mathrm{~nm}$. If the laser intensity profile does vary considerably (the burn patterns indicate hot spots primarily at the beam edges) the detector aperture may be sampling a local region of increased excitation probability and the numbers become more meaningful as a relative index and less accurate as an absolute measurement.

The absorption band contour may change appreciably in the free jet expansion as compared to the room temperature bulb experiment used to determine absolute cross sections ( see ref. 10). At longer wavelengths where the transitions are structured (discrete), the interaction width can collapse down to a very narrow range. The absorption profile becomes a sharp spike with a homogeneous linewidth governed by its natural lifetime. Most of the excimer light will be unable to interact with molecule and the effective cross section, as averaged over the excimer pulse, will be greatly reduced. In this situation the absolute quantum yields can be very inaccurate, though the absolute photodissociation cross sections will be independently accurate because of the mulitiplication/division procedure used in their determination. The detailed significance of the observed trend will be discussed in relation to relaxation pathways and radiationless transition theory. 


\section{Experimental Data}

\section{Pyrazine Photodecomposition following 308nm Excitation}

A Lumonics $\mathrm{XeCl}$ Excimer laser which had been in storage for four years was borrowed from Prof. Neumark's lab and rejuvenated to perform the this experiment. The modest powers obtainable $(34 \mathrm{~mJ} /$ pulse at the output coupler, $16 \mathrm{~mJ}$ in the interaction region) were sufficient due to the strong vibronic coupling associated with the pyrazine ${ }^{1} \mathrm{~B}_{3 \mathrm{u}} \leftarrow^{1} \mathrm{~A}_{1 \mathrm{~g}}\left(\mathrm{n} \pi^{*}\right.$ ) transition, $\sigma=6.6 \times 10^{-19} \mathrm{~cm}^{2}$. This cross section was obtained by convoluting the absorption profile from reference 10 (see figure 1- 5) with the excimer output measured by Lambda Physik (see figure 1-6). The absolute quantum yield $\Phi$ was determined by a method of ratios: since the value was so small a hole burning experiment would be difficult; however, comparing the product flux into a similar channel measured at a different wavelength would give the ratio of the product yields which could be then directly converted. For example, both $248 \mathrm{~nm}$ and $308 \mathrm{~nm}$ excitation give rise to $\mathrm{HCN}+\mathrm{C}_{3} \mathrm{H}_{3} \mathrm{~N}$ with about $10 \mathrm{kcal} / \mathrm{mol}$ of available translational energy. Running under the same conditions (beam seed ratio and backing pressure, angle, excitation probability, number of sweeps) gives a $308 \mathrm{~nm}$ peak with $1 / 42$ the area of the $248 \mathrm{~nm}$ signal (center of mass velocity corrected); hence, the quantum yield into this channel at $308 \mathrm{~nm}$ is $1 / 42$ the size of the $248 \mathrm{~nm}$ channel.

The very low quantum yield following $308 \mathrm{~nm}$ excitation could be a result of an inaccurate determination of the absorption coefficient. The room temperature, "high 
pressure' (10 torr) spectrum taken at 1.25 Angstrom resolution could blur very sharp (discrete) vibronic transitions whose linewidth is closer to $0.05 \mathrm{~cm}^{-1}$ (the inverse of the 100 picosecond fluorescence lifetime, or about $1 / 2000$ of a nanometer near $308 \mathrm{~nm}$ ). The two $308 \mathrm{~nm}$ excimer lines fall chiefly on the $9 a_{0}^{1} 16 b_{0}^{2}$ and $6 a_{0}^{2} 10 a_{0}^{1}$ pyrazine absorptions, which seem superimposed on a continuum absorption (see figure 1-5). One can estimate the degree of line narrowing based upon the inverse of the absolute quantum yield, approximately $1 / 300$ of the excimer pulse width (about $0.12 \mathrm{~nm}$, see figure 1-6). This latter value, about $0.05 \mathrm{~cm}^{-1}$, is similar to the estimate from the fluorescence lifetime.

Another reason for the low yield could be related to the dissociation lifetime on the ground state surface. Pyrazine possesses 10 atoms and $24(3 \mathrm{~N}-6)$ vibrational modes which will share the photon energy once intersystem crossing from $T_{1}$ occurs, approximately 10-100 nanoseconds following photoexcitation (ref. 11). Intramolecular vibrational energy redistribution will statistically repartition this excess energy until a critical configuration or transition state which allows passage into a product channel is accessed. The time scale for this reaction process can be estimated by RRKM (RiceRamsberger-Kassel-Marcus) theory. Using the vibrational frequencies of pyrazine (ref.15) and the RRKM program the variation in reaction lifetime versus barrier height was calculated over a range of energies (see figure 1-7). The results demonstrate how a modest barrier of $60 \mathrm{kcals}$ is sufficient to slow the reaction to the millisecond time scale once the ground state is reached. As seen from an excited surface, the same reaction 
barrier is overcome with an increased efficiency of about six orders of magnitude. As we expect the aromatic $\mathrm{C}-\mathrm{C}, \mathrm{C}-\mathrm{N}$ and $\mathrm{C}-\mathrm{H}$ bonds to lie in the 70-100 kcal range, one can appreciate the strong energy dependence these calculations suggest. The energetic accessibility of the transition state, both in terms of absolute energy and relative (barrier) height above the electronic state surface, dramatically influence the reaction lifetime. The excited surface, whose unique potential energy function will determine the configuration space sampled by the excited molecule, can thus facilitate reactions with order of magnitude variations in the rates.

A single channel was positively identified as a primary photoproduct, peaked away from zero translational energy release, indicative of a concerted process. The intensity dependence was checked for both fragments, over the range of excitation probabilities 0.15 to 0.04 . For the HCN fragment this was done by monitoring the $\mathrm{m} / \mathrm{e}=27$ signal directly, for $\mathrm{C}_{3} \mathrm{H}_{3} \mathrm{~N}$ by monitoring the $\mathrm{m} / \mathrm{e}=26$ fragments. A linear power dependence was observed, supportive of a one photon process. Both the $\mathrm{m} / \mathrm{e}=53$ $\left(\mathrm{C}_{3} \mathrm{H}_{3} \mathrm{~N}\right)$ fragment and the $\mathrm{m} / \mathrm{e}=52\left(\mathrm{C}_{3} \mathrm{H}_{2} \mathrm{~N}^{+}\right)$ion were recorded and momentum matched to the HCN partner. The signal to noise for the $m / e=52$ is approximately a factor of four greater than $\mathrm{m} / \mathrm{e}=53$ due to the ionization and fragmentation behavior.

The peak appearing at the beam velocity presents some difficulty. At first glance it would appear to be a $\mathrm{m} / \mathrm{e}=53$ fragment peaking near zero translational energy. Several inconsistencies, most notably the lack of a momentum matched $\mathrm{HCN}(\mathrm{m} / \mathrm{e}=27)$ partner, require explanation. Further widening our inquiry, we note that the intensity ratios of $\mathrm{m} / \mathrm{e}=53$ to $\mathrm{m} / \mathrm{e}=52$ match that of parent pyrazine rather than a $\mathrm{C}_{3} \mathrm{H}_{3} \mathrm{~N}$ fragment. 
Moreover, there is no discernible signal at other masses such as $\mathrm{m} / \mathrm{e}=40$, which could pick up intensity due to 'hot' pyrazine ionizing and then dissociating. From our knowledge of fragmentation patterns we can rule out a dissociation into $\mathrm{m} / \mathrm{e}=26$ (acetylene) $+\mathrm{C}_{2} \mathrm{H}_{2} \mathrm{~N}_{2}$, due to lack of signal at $\mathrm{m} / \mathrm{e}=27$. The possibility of either $\mathrm{H}$ or $\mathrm{H}$ ${ }_{2}$ elimination yielding a heavy fragment at $\mathrm{m} / \mathrm{e}=79$ or 78 which further cracks is considered based upon the following evidence. Both $\mathrm{m} / \mathrm{e}=1$ and $\mathrm{m} / \mathrm{e}=4\left(\mathrm{D}_{2}\right.$ from pyrazine- $\mathrm{d}_{4}$ ) signals were measured and found to be statistically insignificant at $308 \mathrm{~nm}$. These scans were 1.3 and 1.8 million scans long, respectively, at excitation probability 0.04. Based upon the yields into the $53 / 52 / 26$ channels one can estimate a branching ratio of about 0.3 compared to the fast $\mathrm{HCN}$ channel, or a quantum yield of 0.0008 \pm .0004 . From the observed signals, excitation probabilities and branching ratios for $\mathrm{H}$ and $\mathrm{H}_{2}$ elimination at 248 and $193 \mathrm{~nm}$ the $308 \mathrm{~nm}$ signal will be roughly three orders of magnitude weaker and thus could be easily hidden within the 'noise' fluctuations. The intensity of the $\mathrm{m} / \mathrm{e}=53$ and $\mathrm{m} / \mathrm{e}=52$ peaks present the strongest challenge: the strongest fragment is expected to result from $\mathrm{HCN}$ loss and thus be found at $\mathrm{m} / \mathrm{e}=52$, not $\mathrm{m} / \mathrm{e}=53$. The existence of the $\mathrm{m} / \mathrm{e}=53$ peak alone discounts the plausibility of a $\mathrm{m} / \mathrm{e}=78$ fragmentation pathway and severely reduces support of a $\mathrm{m} / \mathrm{e}=79$ channel.

Another possible explanation for this slow peak is from dimer contributions. Beam conditions were chosen to greatly minimize dimer concentration based upon dimer fragmentation measurements (off axis signals at $\mathrm{m} / \mathrm{e}=80$ (parent),53,27,26) and were ultimately confirmed by noting the similarity of these signals when heating the nozzle to $600 \mathrm{~K}$. Since the yield into this channel is so small perhaps it represents the fraction of 
dimers in the beam ( 1 part in 1250). The strongest evidence against this possibility was a 1.4 million sweep run done under identical molecular beam and detector conditions at $\mathrm{m} / \mathrm{e}=80$ with $248 \mathrm{~nm}$ excitation. No discernible signal above background suggested a dimer concentration at least two to three orders of magnitude less than $1: 10^{3}$.

A likely explanation for this peak is photon sensitized parent ion fragmentation. This could be due to several mechanisms such as isomerization before ionization or dissipation of excess internal energy after ionization. From its inferred quantum yield $(0.0008)$ it is a relatively weak effect, but one important to consider and quantify when discussing products appearing at the beam velocity. Photoisomerization studies done following $S_{1}$ excitation $\left({ }^{1} B_{3 u} \leftarrow^{1} A_{1 g}\right.$ ) (ref. 12-13) find conversion efficiencies on the order of $2-5 \times 10^{-3}$ and the most probable isomer, 1,3 diazine (pyrimidine) shows a mass spectral pattern virtually identical to pyrazine. Other isomers such as 1,2 Diazine (pyridazine) do not yield fragments at 53 readily, and succinonitrile (butanedintrile) will give a prominent $\mathrm{m} / \mathrm{e}=53$ peak (six times more intense than the parent!), a $\mathrm{m} / \mathrm{e}=52$ peak $1 / 3$ as intense, but also a significant peak at $m / e=40(2 / 3$ of the $m / e=53$ intensity) which was simply not observed. More exotic isomers such as the diaza-benzvalene or dewar pyrazine cannot be ruled out immediately but certainly require stronger justification and evidence. The latter explanation, that of dissipating excess energy upon ionization via fragmentation, is cautiously favored.

The third series of peaks observed were associated with elimination of fast acetylene $(\mathrm{m} / \mathrm{e}=26)$. This was confirmed by taking the spectrum at $\mathrm{m} / \mathrm{e}=25$ which 
eliminated the influence of $\mathrm{m} / \mathrm{e}=27(\mathrm{HCN})$ cracking. The most notable feature of these energetic peaks was their intensity dependence, which showed non-linear but nonquadratic behavior. The most straight forward explanation was that the beam intensity was not uniform and the hot spots and diffraction edges were already significantly saturating the excitation process. The beam profile from the Lumonics laser was generally poorer (less uniform) than the Lambda Physik counterpart and showed notable structure at the focal region.

Another possibility worth exploring is that of a strongly enhanced second cross section. The selection rules for two photon absorption (enforcing strong concordance with the symmetry propagator) show

$$
\Gamma_{f} \otimes \Gamma_{\mu} \otimes \Gamma_{m} \otimes \Gamma_{m} \otimes \Gamma_{\mu}^{\prime} \otimes \Gamma_{i}=\Gamma_{f} \otimes \Gamma_{\mu} \otimes \Gamma_{\mu} \otimes \Gamma_{i} \supset A_{1 \varepsilon}
$$

where $\mid \mathrm{m}>$ is dominated by the ${ }^{1} \mathrm{~B}_{3 \mathrm{u}}$ (symmetry allowed) intermediate and $\mu, \mu^{c}$ are the dipole operators $\left(\mu^{c}=\mu_{x} \leftrightarrow b_{3 u}\right)$. This greatly simplifies the analysis as the two photon processes which will transform like the product of coordinates are reduced to ones with at least one $x$ as a factor (e.g. $x^{2}, x y, x z$.) The doubly excited $\left(\mathrm{n} \pi^{*}\right)^{2}, 2{ }^{1} \mathrm{~A}_{\mathrm{lg}}$ state is expected to have two similar (stepwise) oscillator strengths which can not easily account for the order of magnitude change in the second absorption coefficient $\left(\sigma_{2} \approx 20 \sigma_{1}\right.$ based upon the near saturation observed of the total process at a first excitation probability of 
0.09.) Transitions of this intensity $\left(f\left(\sigma_{2}\right)-0.1\right)$ are usually dipole allowed, and will have the symmetry ${ }^{1} \mathrm{~A}_{1 \mathrm{~g}},{ }^{1} \mathrm{~B}_{1 \mathrm{~g}}$ or ${ }^{1} \mathrm{~B}_{2 \mathrm{~g}}$ as dictated by the direct product

$$
b_{3 u} \otimes\left(\begin{array}{l}
b_{3 u} \\
b_{2 u} \\
b_{1 u}
\end{array}\right)=\left(\begin{array}{l}
a_{1 g} \\
b_{1 g} \\
b_{2 g}
\end{array}\right)
$$

Of the ${ }^{1} A_{1 g}$ designation may be states such as the $3 s \leftarrow n$ Rydberg series or high lying $\pi$ states (also of $b_{1 g}$ and $b_{2 g}$ symmetry). As it is known that the ratio of $n \pi: \pi \pi$ transition dipoles are typically 1:10 (ref. 14) there is the possibility of increased transition intensity. The aromatic molecule benzene has excited state cross sections at 248 and $193 \mathrm{~nm}$ estimated to be an order of magnitude higher than the ground state absorption (ref. 35.)

Of considerable interest is also the possibility of a triplet-triplet absorption. It is known that the excited singlet ${ }^{1} \mathrm{~B}_{3 \mathrm{u}}$ has an intersystem crossing rate of $10^{10} \mathrm{~s}^{-1}$ (ref. 10) so the dominant excited species present in a $15 \mathrm{~ns}$ long excimer pulse will be triplet in character. The predominant states involved are thought to be the nearby ${ }^{3} \mathrm{~B}_{2 \mathrm{u}},{ }^{3} \mathrm{~B}_{1 \mathrm{u}}$ and lowest ${ }^{3} \mathrm{~B}_{3 u}$ states. These $\pi \pi$ triplets have strong dipole allowed transitions (for example, considering $\left.{ }^{3} \mathrm{~B}_{2 u}\right)$ to ${ }^{3} \mathrm{~B}_{3 \mathrm{~g}},{ }^{3} \mathrm{~A}_{1 \mathrm{~g}}$ and ${ }^{3} \mathrm{~B}_{1 \mathrm{~g}}$ states. For example, the transition from the triplet manifold to ${ }^{3} \mathrm{~B}_{3 \mathrm{~g}}$ carries an oscillator strength $f-0.1$ and occurs at $64000 \mathrm{~cm}^{-1}$ $\left(T_{00}\right)$ (ref. 15), and there are likely numerous other states in the $50000-65000 \mathrm{~cm}^{-1}$ range which could facilitate a second absorption. The natural lifetime of the upper state is likely much shorter the $S_{1}$ state originally reached, so the effective absorption could interact with a larger portion of the excimer pulse, leading to a very large effective cross 
section. Rapid passage onto the long-lived triplet manifold could conceivably have profound influence on radiative absorption as well as chemical dynamics.

The photoproducts consist of two acetylene $\left(\mathrm{C}_{2} \mathrm{H}_{2}\right)$ channels of almost equal probability, with their sum roughly equal to the single $\mathrm{HCN}$ channel. Because the signal levels were so low the uncertainty in the branching ratio as well as detailed thermodynamic parameters were large. Table 4 below summarizes these results (see figures 1-8 through 1-12 for time-of-flight and detailed fits, and figure 1-13 for characterization of intensity dependence.) Absolute photodissociation cross sections into the one photon channels are obtained by multiplying the quantum yield by the absorption cross section, $\sigma(308 \mathrm{~nm})=6.6 \times 10^{-19} \mathrm{~cm}^{2}$, obtained from ref. 10 .

Table 4. Photodissociation Products, Yields and Energetics Following 308nm Excitation

\begin{tabular}{l|llll}
\hline Reaction & Quantum Yield & Number of & $\left\langle E_{\text {trans }}\right\rangle$ & $E_{\text {transmax }}$ \\
Channel & $\Phi(308 \mathrm{~nm})$ & Photons & $k \mathrm{kal} / \mathrm{mol}$ & $k \mathrm{kal} / \mathrm{mol}$ \\
\hline $\mathrm{HCN}+\mathrm{C}_{3} \mathrm{H}_{3} \mathrm{~N}$ & $3.0 \pm 1.0 \times 10^{-3}$ & $1\left({ }^{1} \mathrm{~B}_{3 \mathrm{u}}\right)$ & $12 \pm 2$ & $23 \pm 5$ \\
$\mathrm{C}_{2} \mathrm{H}_{2}+\mathrm{C}_{2} \mathrm{H}_{2} \mathrm{~N}$ & $1.5 \pm 1.0 \times 10^{-3}$ & $2\left({ }^{3} \mathrm{~B}_{3 \mathrm{~g}} ?\right)$ & $34 \pm 5$ & $60 \pm 15$ \\
$\mathrm{C}_{2} \mathrm{H}_{2}+\mathrm{C}_{2} \mathrm{H}_{2} \mathrm{~N}$ & $1.5 \pm 1.0 \times 10^{-3}$ & $2\left({ }^{3} \mathrm{~B}_{3 \mathrm{~g}} ?\right)$ & $13 \pm 3$ & $25 \pm 5$ \\
$\mathrm{C}_{4} \mathrm{H}_{4} \mathrm{~N}_{2}{ }^{*}$ & $1.0 \pm 0.5 \times 10^{-3}$ & $1\left({ }^{\mathrm{l}} \mathrm{B}_{3 \mathrm{u}}\right)$ & $0 \pm 1$ & $0 \pm 2$ \\
\hline
\end{tabular}

\section{Pyrazine Photodecomposition following 248nm Excitation}


A Lambda Physik EMG-103 excimer with a standard $\mathrm{Kr} / \mathrm{F}_{2} / \mathrm{He}$ fill giving light centered around $248.3 \mathrm{~nm}$ was focused into a $5 \times 2 \mathrm{~mm}$ rectangular spot to define the interaction region. The dramatic increase in product formation is reflected in the numerous channels which bloomed following ${ }^{1} B_{2 u} \leftarrow{ }^{1} A_{1 g}$ excitation. The role of a chemical transformation as a relaxation mechanism was presaged by the two order of magnitude increase in isomerization yield, several order of magnitude drop in fluorescence yield, and order of magnitude drop in phosphorescence yield (compared to ${ }^{3} B_{3 u}$ excitation; see ref. 11). The channels observed are summarized in Table 5 below. Absolute photodissociation cross sections are obtained by multiplying the quantum yields by the absorption cross section $\sigma(248 \mathrm{~nm})=5.0 \times 10^{-18} \mathrm{~cm}^{2}$ (ref. 10). The various spectra and distributions used to characterize the product distribution are presented in figures 1-15 through 1-29, and the intensity dependence of the major channels in figure 1-14. The branching ratios were determined by estimation of ionization cross sections based upon additive atomic polarizabilities (ref. 33).

Table 5. Photodissociation Products, Yields and Energetics Following 248nm Excitation

\begin{tabular}{l|lclll}
\hline Products & Channel & $\Phi\left(\Sigma \Phi_{i}=0.7\right)$ & $\left\langle E_{\text {trans }}\right\rangle$ & $\left\langle E_{\text {trans,max }}\right\rangle$ & Assignment \\
& & & $k c a l / m o l$ & $k c a l / m o l$ & \\
\hline $\mathrm{C}_{4} \mathrm{H}_{3} \mathrm{~N}_{2}+\mathrm{H}$ & 1 & $0.110 \pm .010$ & $9.2 \pm .5$ & $32.0 \pm 10.0$ & pyrazyl \\
$\mathrm{C}_{4} \mathrm{H}_{2} \mathrm{~N}_{2}+\mathrm{H}_{2}$ & 2A & $0.012 \pm .002$ & $31.0 \pm 2.0$ & $45.0 \pm 35.0$ & pyrazyne
\end{tabular}




\begin{tabular}{|c|c|c|c|c|c|}
\hline $\mathrm{C}_{4} \mathrm{H}_{2} \mathrm{~N}_{2}+\mathrm{H}_{2}$ & $2 \mathrm{~B} / \mathrm{C} / \mathrm{D}$ & $0.018 \pm .002$ & $15.0 \pm 5.0$ & $28.0 \pm 5.0$ & vibn. prog. \\
\hline $\mathrm{C}_{3} \mathrm{HN}_{2}+\mathrm{CH}_{3}$ & $15 \mathrm{~A}$ & $0.010 \pm 0.005$ & $3.0 \pm 0.5$ & $12 \pm 1$ & $\begin{array}{l}\text { pyrimidine } \\
\text { only }\end{array}$ \\
\hline $\begin{array}{l}\mathrm{C}_{2} \mathrm{H}_{2} \mathrm{~N}_{2} \\
+\mathrm{C}_{2} \mathrm{H}_{2}\end{array}$ & $26 \mathrm{~A}$ & $<0.020 \pm .010$ & $20.5 \pm 1.0$ & $33.0 \pm 10.0$ & $2 h v$ \\
\hline $\begin{array}{l}\mathrm{C}_{2} \mathrm{H}_{2} \mathrm{~N}_{2} \\
+\mathrm{C}_{2} \mathrm{H}_{2}\end{array}$ & $26 \mathrm{~B}$ & $<0.050 \pm .020$ & $10.8 \pm 2.0$ & $20.0 \pm 5.0$ & $2 h v$ \\
\hline $\begin{array}{l}\mathrm{C}_{3} \mathrm{H}_{3} \mathrm{~N}^{+} \\
\mathrm{HCN}\end{array}$ & $27 \mathrm{~A}$ & $0.400 \pm .010$ & $24.3 \pm 2.0$ & $53.0 \pm 10.0$ & $\begin{array}{l}\text { monoazete } \\
\text { (triplet) }\end{array}$ \\
\hline $\begin{array}{l}\mathrm{C}_{3} \mathrm{H}_{3} \mathrm{~N}+ \\
\mathrm{HCN}\end{array}$ & $27 \mathrm{~B}$ & $0.080 \pm .002$ & $7.1 \pm 1.0$ & $16.0 \pm 4.0$ & $\begin{array}{l}\text { monoazete } \\
\text { (singlet) }\end{array}$ \\
\hline $\mathrm{C}_{3} \mathrm{H}_{3} \mathrm{~N}^{+}$ & $27 \mathrm{C}$ & $0.030 \pm .010$ & $0.080 \pm .010$ & $4.0 \pm 2.0$ & ground state \\
\hline $\mathrm{HCN}$ & & & & & fragment \\
\hline $\mathrm{C}_{4} \mathrm{H}_{4}+\mathrm{N}_{2}$ & 28 & $<0.020 \pm .010$ & $15.0 \pm 1.0$ & $32.0 \pm 5.0$ & $2 h v$ \\
\hline $2 \mathrm{C}_{2} \mathrm{H}_{2} \mathrm{~N}$ & $40 \mathrm{~A}$ & $0.042 \pm .008$ & $3.4 \pm 0.4$ & $13.0 \pm 3.0$ & ground state \\
\hline $2 \mathrm{C}_{2} \mathrm{H}_{2} \mathrm{~N}$ & $40 \mathrm{~B}$ & $0.014 \pm .003$ & $1.8 \pm 0.2$ & $3.0 \pm 0.5$ & ground state \\
\hline $\begin{array}{l}2 \mathrm{HCN}+ \\
\mathrm{C}_{2} \mathrm{H}_{2}\end{array}$ & $\mathrm{~T} 1$ & $<0.02 \pm .01$ & 10/frag. & $45.0 \pm 5$ total & triple \\
\hline
\end{tabular}

a). $\mathrm{H}$ atom elimination 
The highest resolution $\mathrm{m} / \mathrm{e}=1$ spectrum was taken with the detector at right angles to the molecular beam velocity with the excimer laser intersecting an unskimmed beam $10 \mathrm{~mm}$ from the expansion hole. Previous studies with this configuration confirmed the ability to resolve the vibrational structure of $\mathrm{H}_{2} \mathrm{~S}$ photodissociation products and resolve sharp peaks from $\mathrm{HI}$ and DI photofragmentation. The difficulty in measuring the $\mathrm{m} / \mathrm{e}=79$ partner was evident for reasons described in the earlier section, namely the unfavorable translational energy partitioning into the heavy fragment and large background from parent ionization and fragmentation. The spectrum could be fit with the three basis functions (channels) from $\mathrm{H}_{2}$ elimination so a long scan at $\mathrm{m} / \mathrm{e}=79$ was performed to confirm that the signal wasn't from cracking of molecular hydrogen. The weak signal at $\mathrm{m} / \mathrm{e}=79$ suggests that the pyrazyl formed may readily fragment further. The higher count levels $\left(\# \mathrm{~m} / \mathrm{e}=1(\mathrm{H})>\# \mathrm{~m} / \mathrm{e}=4\left(\mathrm{D}_{2}\right)\right.$, Jacobian corrected), the faster $\mathrm{H}$ peak at $193 \mathrm{~nm}$, and the similar spectrum observed in s-triazine confirmed the predominance of atomic hydrogen loss. The first notable feature of the spectrum, a fast peak, may have a slower shoulder but cannot be readily resolved due to the signal/noise level and thus is fit with a single channel. The slower, broad envelope is fit well with the distribution of fast $\mathrm{HCN}$ elimination and may effectively cover up a slower $\mathrm{H}$ loss channel or tail. The large yield into this channel is attributed to the expected stability of the pyrazyl radical, whose $\Delta \mathrm{H}_{\mathrm{f}}^{\mathrm{o}}$ can be derived from the maximum translational energy release using

$$
\Delta \mathrm{H}_{\mathrm{f}}^{\circ}(\text { pyrazyl })=\Delta \mathrm{H}_{\mathrm{f}}^{\circ}(\text { pyrazine })-\Delta \mathrm{H}_{\mathrm{f}}^{\mathrm{o}}(\mathrm{H})+\mathrm{h} v-\mathrm{E}_{\text {trans,max }}
$$


and is found to be $78 \pm 10 \mathrm{kcal} / \mathrm{mol}$. A comparison to the pyridyl radical $\left(\Delta \mathrm{H}_{\mathrm{f}}{ }^{\circ}=90.2\right.$ kcal, ref. 16) suggests an increased stability, due in part to the $\mathrm{C}-\mathrm{H}$ bond which is weakened by the inductive effect of the nitrogen substituents. The relatively large uncertainty results from the experimentally determined realization that multiphoton processes will give rise to faster $\mathrm{H}$ atom products. Although conditions are chosen to minimize this contribution (first excitation probabilities of 0.06 ), because it will appear on the fast tail of the $P(E)$ a small contribution can easily provide a few percent of the counts which stretch the tail significantly. Evidence from intensity dependence studies and photographic burn patterns suggest that the beam profiles are slightly non-uniform, which simply calls for caution in interpreting properties of certain fast tails. Possible excited state absorption may be enhanced relative to the ground state transition by lifetime broadening, as may have occurred following $308 \mathrm{~nm}$ excitation. The method that had been used for years to measure light intensity, collecting the light exiting a small output window after it had gone through a focus and then expanded, was found to be systematically low by about a factor of 3 for $248 \mathrm{~nm}$ and up to a factor of 10-20 for $193 \mathrm{~nm}$ light. A more accurate method (aligning the pyrolytic detector inside the vacuum chamber) was used consistently in this study.

\section{b). $\mathrm{H}_{2}$ Elimination}

Considerable time was spent obtaining a well-resolved spectrum at $\mathrm{m} / \mathrm{e}=4$ (D) $)$ approximately 9 hours to take a 1.5 million sweep scan. Because of high $\mathrm{H}$ background levels a small sample of (deuterated) pyrazine-d 4 (Aldrich, 98\% D atom) was used 
without further purification, as part of a 50/50 mixture of pyrazine- $h_{4}$ that would allow meaningful comparison of $\mathrm{H} / \mathrm{D}_{2}$ signal levels. The mixture would also provide a means of optimizing conditions to see the relatively strong $\mathrm{H}$ atom signal and then shifting to look for $\mathrm{D}_{2}$ before the sample ran out. A model 10 quadrupole head $(\mathrm{m} / \mathrm{e}=0-8)$ was used to obtain improved resolution and transmission. The beam was seeded in neon because helium would give a large $\mathrm{m} / \mathrm{e}=4$ signal and beam parameters (velocity, speed ratio) were measured with the regular (skimmed) source at a variety of backing pressures and then applied to the $90^{\circ}$ configuration.

The structure observed in the time-of-flight was found to be peaks evenly spaced by about $8-9 \mathrm{kcal}$ in energy space. The vibrational frequency of $D_{2}\left(2990 \mathrm{~cm}^{-1}\right.$,ref. 17$)$ predicts a spacing of $8.5 \mathrm{kcal}$. Detailed inspection of the signal trace supports assignment of a progression spanning 4-5 quanta, with the fastest peak showing a long tail. In fitting the $\mathrm{m} / \mathrm{e}=78$ cofragment only two fast traces match, consistent with the $\mathrm{H}_{2}$ stretch frequency of $4160 \mathrm{~cm}^{-1}$ (ref. 17) The signal to noise of this latter trace is not good enough to warrant further analysis except that the profile shows a larger contribution peaked near the beam velocity, likely from $\mathrm{m} / \mathrm{e}=79 \mathrm{cracking}$ in the ionizer to give $\mathrm{m} / \mathrm{e}=78$.

If we assign the maximum in the intensity distribution as $v=0$ quanta of $D_{2}$ stretch then the progression $v=2$ (channel 2-B), $v=3(2-C), v=4(2-D)$ follows. The fast tail in 2-A seems to large to be from multiphoton processes by could conceivably be the result of $\mathrm{D}_{2}$ elimination from species such as the monoazete or acrylonitrile converting to cyanoacetylene or the ground state (linear) pyrazine becoming butenedinitrile. 
Energetic considerations show that all of these pathways are accessible thermodynamically, based upon the heat of formation of cyanoacetylene $(84.6 \mathrm{kcal}$, ref. 18) and butenedinitrile ( $81.3 \mathrm{kcal}$, ref. 4). Assuming a second (fast) component to the 2-A peak, the heat of formation of pyrazyne is estimated at $116-35 /+5 \mathrm{kcal} / \mathrm{mol}$, with the lower uncertainly coming from the anomaly in the fast tail. In comparison, benzyne has an experimental estimate for its $\Delta \mathrm{H}_{\mathrm{f}}^{\circ}$ of $118 \pm 5 \mathrm{kcal} / \mathrm{mol}$ (ref. 19). Ab initio work done on benzyne and related systems (ref. 20) notes significant biradical character associated with the triply bonded carbons, whose substituents are not at $180^{\circ}$. If the pyrazyne heat of formation is closer to the $80-90 \mathrm{kcal}$ lower limit extensive configuration interaction such as in-plane conjugation of the $\pi$ system predicted for the dehydropyridazines (ref. 20) is possible. From the large fraction of vibrationally excited species measured in the $v=1,2, . .4$ levels a transition state far from the equilibrium geometry of $\mathrm{H}_{2}\left(\mathrm{D}_{2}\right)$ is suspected. The branching ratios into each of these vibronic channels is estimated by the fitting procedure to be: $v=0,1 \approx 1 ; v=2 \approx 0.58$; $v=3 \approx 0.46$; $v=4 \approx 0.39$, with uncertainty in the $20 \%$ range due to overlapping peaks. The hydrogens will arise from a strained geometry imposed by the relatively rigid carbonnitrogen framework, and the in-phase out-of-plane hydrogen bend $\left(v_{10 a}\right)$ can serve as an effective promoting mode to reach the critical geometry.

c). Methyl Elimination 
This channel is observed for 1,3 Diazine (pyrimidine) and efforts to find its contribution to pyrazine dissociation established a lower limit on the order of $0.1 \%$. This is consistent with the relatively minor extent of isomerization observed for the diazines and discussed earlier. The greater prominence of this channel in benzene (4\%, ref. 21$)$ and especially pyridine (21\%@193nm, ref. 22) can be rationalized by considering the availability of hydrogen and the stability of the heavier co-fragment. For pyridine, for example, a 2-butynenitrile radical analogous to a propargyl with a cyano substituent to further conjugate into will enjoy an expected stabilization on the order of $1 \mathrm{eV}$ compared to its benzene analog. The second nitrogen of diazines forces different structural topologies less favorably disposed to eliminating a methyl radical. The translational energy distribution peaked near zero is consistent with a simple bond rupture with a small amount of rehybridization occurring in the two fragments.

\section{d.) $\mathrm{C}_{2} \mathrm{H}_{2}$ (Acetylene) Loss}

In parallel with our treatment following $308 \mathrm{~nm}$ excitation, intensity dependence tests of products showed a non-linear increase for the fast peak 26-A. Channel 26-B was buried underneath the fragmentation of $\mathrm{m} / \mathrm{e}=53$ from channel $27-\mathrm{A}$ and was inferred from the $\mathrm{m} / \mathrm{e}=54$ scan and from $\mathrm{m} / \mathrm{e}=26$ at the highest angles where the peaks slightly separated. The existence of acetylene was verified directly by a m/e=25 $\left(\mathrm{C}_{2} \mathrm{H}^{+}\right)$scan at high electron impact energies $(200 \mathrm{eV})$ that matched features in the $\mathrm{m} / \mathrm{e}=26$ peak well. Interference from $\mathrm{HCN}$ cracking to $\mathrm{CN}+$ was expected and observed with a 0.067 fractional efficiency, which accounted for the slightly less than quadratic dependence in 
the $\mathrm{m} / \mathrm{e}=26$ signal. Scans at $\mathrm{m} / \mathrm{e}=25$ typically took $8-20$ hours so only selective ones were performed. The low yields and two photon requirement for this fragment is thought to originate from the higher energy dewar intermediate that must be formed following isomerization through the benzvalene. If a $\mathrm{C}_{2} \mathrm{H}_{2}$ loss channel peaked near the beam velocity were present it would be effectively hidden by the other slow products which readily fragment to $m / e=26$, unless this channel comprised more than about $3 \%$ of total reactive signal.

\section{E. HCN Elimination}

Elimination of hydrogen cyanide ( $\mathrm{HCN})$ is the predominant photoreaction irthe mono, di- and tri-azabenzenes. That three distinct distributions (referred to as channels 27-A, 27-B, 27-C) of $\mathrm{HCN}$ would be produced in pyrazine may not be easy to believe. The main justification given in three parts: (1) careful inspection of the time-of-flight spectra at $\mathrm{m} / \mathrm{e}=53,52,27,26,25$, noting the shoulders especially on the $\mathrm{m} / \mathrm{e}=53,52$ peaks

(2) quantitative knowledge of the fragmentation characteristics of this series (3) intensity dependent scans showing the preferential depletion of channels 27-A and 27-C (see figure 1-30). Had a typical speed ratio beam (s 15) been used channels 27-A and 27-B most probably would be unresolvable. From the maximum translational energy release the heat of formation of the monoazete can be estimated at $78 \pm 10 \mathrm{kcal}$. The slowest channel (27-C) is thought to originate following rearrangement of a linear species, which is preceded by ring opening. The relatively small yield into this channel is 
thus an indication of the inefficiency of the system to access the ground state (or a biradical linear form) following $S_{2}$ excitation.

The two faster channels are thought to originate from the excited manifold of singlet and triplet surfaces associated with pyrazine and its valence isomers, most notably benzvalene, dewar-pyrazine and fulvene (or prefulvene). The two channels correlate with the two different spin-states of the monoazete product which are energetically similar ( $\Delta \mathrm{E}$ singlet-triplet $\sim 1 \mathrm{eV}$ from $a b$ initio calculations described later.)

f.) Nitrogen Loss $(\mathrm{m} / \mathrm{e}=28$, channel $28-\mathrm{A})$

Photoisomerization of 1,2 diazine yields both 1,3 and 1,4 diazine. By the principle of microscopic reversibility, the production of 1,2 diazine from isomerization of pyrazine ( 1,4 form) is realizable. In a cycle similar to the process leading to $\mathrm{C}_{2} \mathrm{H}_{2}$ elimination, two nitrogens can also come together via the benzvalene pathway and then be eliminated. As expected, the yield into this pathway is small and likely requires multiple photons. The possibility of a rearrangement occuring through a prismane intermediate is much more energetically costly and less likely based upon the apparent reactivity of the dewar intermediate.

The strongest evidence is the scan taken at $\mathrm{m} / \mathrm{e}=49$ (where neither azete can populate via sequential $\mathrm{H}$ loss) and a very long scan taken at $\mathrm{m} / \mathrm{e}=28$ (where the background levels due to $\mathrm{CO}$ make detection difficult). High resolution mass spectral analysis of parent fragmentation revealed that $\mathrm{N}_{2}$ is not eliminated from pyrazine following dissociative ionization; the extent of rearrangement is apparently too great. 
The method of using ${ }^{15} \mathrm{~N}$ substituted pyrazine has been suggested as the most conclusive manner to confirm this channel. Only the fastest peak (28-A) is fit because the two slower ones could conceivably arise from fragmentation of precursors (into $\mathrm{CH}_{2} \mathrm{~N}$, for example). The 28-A nitrogen peak can not be fit by considering the ${ }^{15} \mathrm{~N}$ contribution from $\mathrm{HCN}$ both intensity and energy-wise. It is the most compelling evidence of the extent of isomerization within the carbon-nitrogen ring.

g.) Cyanomethylene, methylene nitrile, or nitrogen substituted propargyl $(\mathrm{m} / \mathrm{e}=40)$ radical

The relatively facile cleavage of the $\mathrm{C}-\mathrm{N}$ bond which converts the ring to a linear form can occur a second time to yield two products at $\mathrm{m} / \mathrm{e}=40$. Either atomic hydrogen elimination or a $[1,2]$ hydrogen shift can induce the production of a stable cyano terminal group and a relatively weak $\mathrm{C}-\mathrm{N}$ single bond, rupture of which will lead to a nitrogen substituted propargyl $\left(\mathrm{CH}_{2} \mathrm{CN}\right)$ and a fragment of the same molecular formula. The high signal/noise attainable in this channel made the resolution of two channels possible, the more translationally energetic one (40-A) thought to originate from identical departing structures that are slightly repulsive along the reaction coordinate (e.g. rehybridization of the electrons in the freshly broken bond). Channel 40-B, which is less favored, results from simple bond rupture of a less repulsive state or from secondary decomposition of the pyrazyl radical. In the former case the two $\mathrm{m} / \mathrm{e}=40$ fragments could come from either singlet or triplet precursors, while in the latter case it would 
require production of $\mathrm{m} / \mathrm{e}=39+40$ fragments that would be formed following dissociative ionization following electron impact because of energetic considerations.

h.) Additional Experiments

Because of the anticipated involvement of the valence isomers, two color experiments were attempted to see if different behavior could be induced by different excitation paths. It is known, for example, that the dewar isomer can only be reached following $S_{2}$ excitation in benzene while the benzvalene and fulvene forms are accessible following $S_{1}$ excitation (ref. 23). If such isomers were long lived then a second photon could potentially induce photodissociation which differed from one photon excitation processes. The method attempted was to reverse the order of light pulse sequence and see if any asymmetry was induced in the products, i.e. the subsequent reactions showed a path dependence. The three wavelengths, 308, 248 and $193 \mathrm{~nm}$ were combined pairwise in different combinations and delays ranging from $20 \mathrm{~ns}$ to $500 \mathrm{~ns}$. The resulting signals at $\mathrm{m} / \mathrm{e}=26,27,40,52$ showed no behavior that was not consistent with additive contributions, although the tests had to be performed in a short amount of time due to the intensive equipment requirements which were could only be borrowed for a short time from other experiments. A similar experiment to measure the time dependence of product formation via second photon depletion through ionization (the azetes have ionization potentials in the $6.5-7 \mathrm{eV}$ range) did not produce a signal that varied with time delay between the pulses as well. Plans to look for the transient ${ }^{1} \mathrm{H} N \mathrm{R}$ traces related to the strong $T_{1}$ triplet state interactions (unpaired electrons with the ring 
protons) were hampered by the technical difficulties of optically pumping a sample spinning in the departmental spectrometers.

Polarization dependence of the fast $\mathrm{m} / \mathrm{e}=1$ and 27 chamels showed differences no greater the $8 \%$ (i.e. $\beta<0.04 \pm 0.04$ ), implying the excited complex lives for several rotational periods before products come off. The calculated rotational period in the $\mathrm{J}=1$ state $\left(B=0.2 \mathrm{~cm}^{-1}\right.$, ref. 15$)$ is 95 picoseconds, suggesting a unimolecular reaction lifetime in the hundreds of picoseconds to nanoseconds range. Values of $\beta$ this small are generally considered at the lower end of what can be considered significant to comment upon. Similar dependence was seen at $193 \mathrm{~nm}$, implying a rather long lived excited state pathway is followed before chemical products emerge.

\section{Pyrazine Photodissociation Products following 193nm Excitation}

The same experimental procedure followed at $248 \mathrm{~nm}$ was used to measure the photoproducts following ${ }^{1} \mathrm{~B}_{1 \mathrm{u}} \leftarrow^{1} \mathrm{~A}_{1 \mathrm{~g}}\left(\pi \pi^{*}\right)$ excitation. The sensitivity of the product distributions to laser intensities above $0.3 \mathrm{MW} / \mathrm{cm}^{2}$ was notable, as the plot of the $\mathrm{m} / \mathrm{e}=26$ trace demonstrates (fig. 1-31). The experimental traces were typically taken with laser power of $300 \mathrm{uJ} / \mathrm{pulse}$ focused into a $4 \times 2 \mathrm{~mm}$ spot, corresponding to a first excitation probability of about $5 \%$ for a uniform beam. This allowed individual scans to be completed within a 3-20 hour time frame, but made potential contributions from two photon processes a concern for certain channels. Since the high intensity behavior was known for these channels, however, the effect was limited to an increased uncertainty in 
the energetic extent of the one photon channel once the linearity of the power dependence was established. The results are summarized in Table 6 below, and the supporting spectra are presented in figures 1-14 and 1-32 through 1-48.

Table 6. Photodissociation Products, Yields and Energetics Following 193nm Excitation

\begin{tabular}{|c|c|c|c|c|c|}
\hline Products & Channel & $\Phi\left(\Sigma \Phi_{i}=0.9\right)$ & $\begin{array}{l}<E_{\text {trans }}> \\
k \mathrm{kal} / \mathrm{mol}\end{array}$ & $\begin{array}{l}<E_{\text {trans,max }}> \\
\text { kcal/mol }\end{array}$ & Assignment \\
\hline $\mathrm{C}_{4} \mathrm{H}_{3} \mathrm{~N}_{2}+\mathrm{H}$ & $1-A$ & $0.240 \pm .020$ & $37.5 \pm 4.0$ & $75 \pm 10$ & pyrazyl+H \\
\hline $\mathrm{C}_{4} \mathrm{H}_{3} \mathrm{~N}_{2}+\mathrm{H}$ & $1-B$ & $0.040 \pm .010$ & $1.0 \pm 1.0$ & $5 \pm 5$ & thermal $\mathrm{H}$ \\
\hline $\mathrm{C}_{4} \mathrm{H}_{2} \mathrm{~N}_{2}+\mathrm{H}_{2}$ & $2-A$ & $0.010 \pm .003$ & $32 \pm 5.0$ & $80 \pm 10$ & pyrazyne \\
\hline $\mathrm{C}_{4} \mathrm{H}_{3} \mathrm{~N}_{2}+\mathrm{H}_{2}$ & $2-B / C$ & $0.005 \pm .003$ & $12 \pm 4.0$ & $25 \pm 10$ & weak vib. \\
\hline $\mathrm{C}_{2} \mathrm{H}_{2} \mathrm{~N}_{2}$ & $26-A$ & $0.030 \pm .020$ & $27.0 \pm 6.0$ & $42 \pm 10$ & diazete + \\
\hline$+\mathrm{C}_{2} \mathrm{H}_{2}$ & & & & & acetylene \\
\hline $\mathrm{C}_{2} \mathrm{H}_{2} \mathrm{~N}_{2}$ & 26-B & $0.010 \pm .005$ & $9.7 \pm 2.0$ & $15 \pm 5$ & diazete + \\
\hline$+\mathrm{C}_{2} \mathrm{H}_{2}$ & & & & & acetylene \\
\hline $\mathrm{C}_{3} \mathrm{H}_{3} \mathrm{~N}$ & $27-A$ & $0.220 \pm .020$ & $23.2 \pm 3.0$ & $53 \pm 10$ & monoazete \\
\hline$+\mathrm{HCN}$ & & & & & $+\mathrm{HCN}$ \\
\hline $\mathrm{C}_{3} \mathrm{H}_{3} \mathrm{~N}$ & $27-B$ & $0.070 \pm .007$ & $7.8 \pm 1.0$ & $16 \pm 3$ & monoazete \\
\hline$+\mathrm{HCN}$ & & & & & $+\mathrm{HCN}$ \\
\hline $\mathrm{C}_{3} \mathrm{H}_{3} \mathrm{~N}$ & $27-C$ & $0.090 \pm .010$ & $0.4 \pm 0.1$ & $1 \pm 0.5$ & acrylonitrile \\
\hline$+\mathrm{HCN}$ & & & & & $+\mathrm{HCN}$ \\
\hline
\end{tabular}




\begin{tabular}{l|lllll}
$\mathrm{C}_{3} \mathrm{H}_{3} \mathrm{~N}$ & $27-\mathrm{D}$ & $0.010 \pm .001$ & $2.7 \pm 0.5$ & $6 \pm 1$ & acrylonitrile \\
$+\mathrm{HCN}$ & & & & & $+\mathrm{HCN}$ \\
$2\left(\mathrm{C}_{2} \mathrm{H}_{2} \mathrm{~N}\right)$ & $40-\mathrm{A}$ & $0.080 \pm .008$ & $6.2 \pm 1.0$ & $14 \pm 2$ & N-propargyl \\
$2\left(\mathrm{C}_{2} \mathrm{H}_{2} \mathrm{~N}\right)$ & $40-\mathrm{B} / \mathrm{C}$ & $0.030 \pm .005$ & $1.8 \pm 0.4$ & $3 \pm 2$ & N-propargyl \\
$2(\mathrm{HCN})$ & $27-\mathrm{T}$ & $<0.050 \pm .020$ & $20 \pm 5 /$ frag & $75 \pm 10$ total & triple diss. \\
$+\mathrm{C}_{2} \mathrm{H}_{2}$ & & & & & \\
\hline
\end{tabular}

Absolute photodissociation cross sections can be obtained by multiplying the quantum yield by the absorption cross section, $\sigma(193 \mathrm{~nm})=6.7 \times 10^{-18} \mathrm{~cm}^{2}$ (ref. 10).

The overall behavior is quite similar to the products of $S_{2}$ excitation. The notable differences are the overall increase in translationally slow products, arising from an increased ring opening efficiency, i.e. improved conversion to either the ground state or a linear (biradical) form. Although the simplest explanation for this would be a direct ring opening, the high intensity data which favors very translationally fast products at the expense of the slower channels seems to support an internal conversion mechanism at least at moderate energies. The delocalized nature of the molecular orbitals involved in the excitation process support the latter hypothesis as well. Whereas only $10 \%$ of the products at $248 \mathrm{~nm}$ populate the channels $40-\mathrm{A} / \mathrm{B} / \mathrm{C}$ and $27-\mathrm{C} / \mathrm{D}$, at $193 \mathrm{~nm}$ the yield increases to about $40 \%$. The fast peaks at $\mathrm{m} / \mathrm{e}=27,26$ and $2\left(\mathrm{HCN}, \mathrm{C}_{2} \mathrm{H}_{2}\right.$ and $\mathrm{H}_{2}$ elimination) show identical characteristics to the energy released at $248 \mathrm{~nm}$, implying that an exit barrier dominates the translational energy partitioning into these channels. 
The contribution at $\mathrm{m} / \mathrm{e}=26$ (confirmed from a $\mathrm{m} / \mathrm{e}=25$ trace) shows a small but nonzero component that cannot be accounted for by fragmentation of $\mathrm{HCN}$, as well as an intensity dependence that mirrors the linear $\mathrm{m} / \mathrm{e}=27$ channels. The $\mathrm{m} / \mathrm{e}=1 \mathrm{H}$ loss peak shifts to higher energies and becomes the predominant hydrogen loss mechanism. In general, simple bond rupture is favored over concerted mechanisms due to the greater amount of available energy and larger A factor of the Arrhenius rate expression:

$k(n, E)=A(E) \cdot \exp \left[\frac{-\Delta E^{\ddagger}}{k_{b} T}\right]$

where $\mathrm{k}$ is the rate constant into channel $\mathrm{n}, \mathrm{A}$ is the frequency factor along the reaction coordinate, $\Delta \mathrm{E}^{*}$ the activation energy, and $\mathrm{k}_{\mathrm{b}}$ Boltzmann's constant. Vestiges of the $248 \mathrm{~nm}$ channels are still very pronounced at a higher excitation energy. The energy level diagram for the photoreactive pathways is presented in figure 1-47.

\section{The Photodissociation of $1,3,5$ Triazine}

Although this molecule had been studied before and its photochemistry 'determined' as triple dissociation from the ground state surface (ref. 24), the possibility of triazine displaying similar characteristics to pyrazine warranted further attention. Many 'new' channels were recognized based upon the pyrazine model and are summarized in table 7 below.. Because of time constraints the quantum yields and intensity dependence were not determined; supporting spectra are presented in figures 148 through 1-53. 
Table 7. Selected Photodissociation Products of 1,3,5 Triazine (248nm excitation)

\begin{tabular}{l|llll}
\hline Products & Branching & $\left\langle E_{\text {trans }}>\right.$ & $<E_{\text {trans,max }}>$ & Assignment \\
& Ratio & $k c a l / m o l$ & $k$ callmol & \\
\hline $\mathrm{C}_{3} \mathrm{H}_{2} \mathrm{~N}_{3}+\mathrm{H}$ & $0.12 \pm .02$ & $9.9 \pm 1.0$ & $31 \pm 5.0$ & triazyl \\
$\mathrm{C}_{2} \mathrm{H}_{2} \mathrm{~N}_{2}+\mathrm{HCN}$ & $0.83 \pm 0.08$ & $28 \pm 3.0$ & $65 \pm 10$ & diazete \\
$\mathrm{C}_{2} \mathrm{H}_{2} \mathrm{~N}_{2}+\mathrm{HCN}$ & $<0.02 \pm 0.01$ & $1.0 \pm 0.5$ & $3.0 \pm 1.0$ & gnd. state \\
$\mathrm{C}_{2} \mathrm{H}_{2} \mathrm{~N}+\mathrm{C}_{1} \mathrm{H}_{1} \mathrm{~N}_{2}$ & $0.05 \pm 0.01$ & $4.0 \pm 1.0$ & $9.0 \pm 2.0$ & $\mathrm{~N}$-propargyl \\
$\mathrm{C}_{2} \mathrm{H}_{2} \mathrm{~N}+\mathrm{C}_{1} \mathrm{H}_{1} \mathrm{~N}_{2}$ & $<0.01 \pm 0.005$ & $0.8 \pm 0.2$ & $3.0 \pm 1.0$ & $\mathrm{~N}$-propargyl \\
$\mathrm{C}_{3} \mathrm{H}_{3} \mathrm{~N}+\mathrm{N}_{2}$ & $<0.03 \pm .02$ & $20.0 \pm 2.0$ & $40.0 \pm 0.2$ & $\mathrm{~N}_{2}+$ monoazete \\
$3(\mathrm{HCN})$ & $<0.10 \pm 0.1$ (est.) & $30.0 \pm 5.0$ & $65.0 \pm 0.2$ & estimated lim. \\
\hline
\end{tabular}

The role of $\mathrm{H}$ atom loss as an initiating step leading to several secondary dissociation processes is suspected for the 'slowest' peaks present. The triazyl radical can ring open directly to the relatively stable cyano radical and further rearrangements can then occur, yielding fragments at $\mathrm{m} / \mathrm{e}=54,53,52,41,40$. The scan at $\mathrm{m} / \mathrm{e}=54$ was taken with an electron impact energy of $30 \mathrm{eV}$ to attempt to preserve the $54+$ fragment but signal still remains very feeble. The signal does identically match the fast $\mathrm{m} / \mathrm{e}=27$ peak and must come from either the 54 fragment or $\mathrm{m} / \mathrm{e}=53$ formed through photoisomerization to $1,2,4$ triazine followed by $\mathrm{N}_{2}$ loss (an additional channel not observed in previous work.) The $\mathrm{N}_{2}$ time-of-flight spectrum was hampered by poor signal/noise but still implies an isomerization yield of at most a few percent; this channel 
is confirmed by the presence of the strong $\mathrm{m} / \mathrm{e}=51$ fragment. The 'tail' of the 27 peak can accommodate a contribution from $\mathrm{m} / \mathrm{e}=54$ cracking to $\mathrm{HCN}^{+}$easily and the $\mathrm{m} / \mathrm{e}=26$ spectrum shows an even greater extent of the fragmentation processes coming presumably from the high mass counterfragments. Mass spectral fragmentation theory would predict that fragments such as $\mathrm{C}_{2} \mathrm{H}_{2}$ would carry away most of the charge as compared to $\mathrm{HCN}$ and $\mathrm{N}_{2}$ molecules, as detailed in figure 1-4.

The many similarities to the pyrazine spectra impl that the behavior of s-triazine may be much more similar than previous studies suggest. The dramatic decrease in HCN translational energy release at $193 \mathrm{~nm}$ is consistent with the significant shift to slow fragments observed in pyrazine (@193nm). Processes capable of opening up the ring system ( $\mathrm{H}$ atom loss followed by opening to the cyano radical, direct $\mathrm{C}-\mathrm{N}$ bond cleavage, internal conversion to $\mathrm{S}_{0}$ followed by simple $\mathrm{C}-\mathrm{N}$ bond rupture) dominate the reaction path and thus lead to slow, heavy products while the special geometries and weakly bound intermediates required for the fast HCN channel are bypassed.

\section{Ab Initio Studies of the Relevant Species in Photodissociation}

The energetic accessibility of the various valence isomers and predicted products provide a significant test to the reaction hypothesis. Earlier studies done with primitive empirical Hamiltonians such as AM1, PM3, and MINDO and were found to be unreliable (ref. 25). For example, AM1 energies of dewar pyrazines were predicted to be lower than the benzvalenes. The work of Gluhovchev et al: (ref. 36) discussed the interconversion of cyclobutadiene and azacyclobutadienes based upon intermediates such 
as 1-azabicyclo[1.1.0]butane-2,4-diradical, noting the decrease in antiaromatic character as nitrogens are substituted into the ring. The relative stability of azetes and their valence isomers such as azatetrahedrane were categorized but limited by the $\mathrm{MINDO} / 3$ method in their accuracy. More current studies focused on cyclobutadiene and trends in structure and antiaromaticity (ref. 26). I went to Dr. Martin Head-Gordon and Dr. A. Streitweiser for consultation and they both agreed that more powerful and accurate methods were available at least for ground state geometries and energies. The 'highest' level of ab initio treatment was 'G2 theory' (ref. 27) which would be difficult to implement for systems bigger than 4 atoms (not counting hydrogens) but slightly less rigorous methods could provide results accurate to about $5 \mathrm{kcal}$.

The procedure used was to optimize an initial geometry at the restricted HartreeFock level using a basis of six primitive Gaussian core orbitals with s and $\mathrm{p}$ valence orbitals and one set of $6 \mathrm{~d}$ functions (referred to in short as RHF/6-31G*). This geometry was then further refined at the MP2 level, meaning Moller-Plesset corrections to second order in energy (first order in the wave function) over an expanded basis including triply split valence orbitals including 2 sets of $\mathrm{d}$ functions, added bases of diffuse orbitals and with polarizability (MP2/6-311+G*) to yield a geometric structure and energy when possible. Full implementation of G2 theory would require calculation at the fourth order of perturbation (MP4SDTQ) which would turn 10 hour computer jobs into month long ventures and hence was not attempted.

Energies were related through isodesmic reactions which fell into two categories: isomerizations and fragmentations. For example, the reference energy of ground state 
pyrazine was calculated at experimentally determined geometries (optimization of this structure yielded a negligible energy change and very small geometry) and all other valence isomers were compared to this value as a measure of $\Delta \mathrm{H}_{\mathrm{iso}}$ :

$$
\mathrm{C}_{4} \mathrm{H}_{4} \mathrm{~N}_{2} \text { (Pyrazine, }{ }^{1} \mathrm{~A}_{1 \mathrm{~g}} \text { ) } \rightarrow \mathrm{C}_{4} \mathrm{H}_{4} \mathrm{~N}_{2} \text { (Dewar Pyrazine, }{ }^{1} \mathrm{~A}_{1} \text { ) }
$$

or for the azetes the $\Delta \mathrm{H}_{\mathrm{rxn}}$ :

$$
\mathrm{C}_{3} \mathrm{H}_{3} \mathrm{~N} \text { (Monoazete, triplet state) } \rightarrow \mathrm{HCN}\left(\mathrm{X}^{-1} \Sigma^{+}\right)+\mathrm{C}_{2} \mathrm{H}_{2}\left(\mathrm{X}^{-}{ }^{1} \Sigma_{\mathrm{g}}^{+}\right)
$$

This last reaction specifies an open-shell species (the triplet azete) which is dealt with by the unrestricted Hartree-Fock (UHF) formalism by calculating separate orbitals for $\alpha$ $(s=+1 / 2)$ and $\beta(s=-1 / 2)$ electrons and satisfying constraints on the spin multiplicity $(2 S+1)$ which is compared to the calculated spin density $\left\langle S^{2}\right\rangle=S(S+1)$ to make sure not too much 'spin contamination' occurs (see ref. 28). Excited state energies were determined by CIS (configuration interaction with single excitation) emphasizing low energy triplet states that could be implicated to the excited triplet manifold. Properties of notable species are presented in Table 8 , with energies relative to the isodesmic reactions described above.

Table 8. Ab Initio Results for Pyrazine Valence Isomers and Derivatives

\begin{tabular}{l|lcl}
\hline Species & RUUHF (kcal) & UMP2 (kcal) & CIS excit. (eV)- Experiment \\
& & & triplet states \\
& 0.0 & 0.0 & $3.4,4.0,4.1$ \\
Pyrazine & 13.9 & 13.9 & $1.9,2.7,3.9$
\end{tabular}




\begin{tabular}{l|llll} 
Benzvalene & 62.2 & 52.7 & $-0.3,2.6,5.0$ & $<89$ (isomerize) \\
Benzvalene(to & 97.3 & - & & \\
1,2 diazine) & & & & \\
Dewar $(\mathrm{HCN})$ & 70.0 & - & $1.9,3.0,3.4$ & \\
Dewar $\left(\mathrm{C}_{2} \mathrm{H}_{2}\right)$ & 113 & - & 3.0, & \\
Dewar $\left(\mathrm{N}_{2}\right)$ & 180 & & & \\
$\left.\mathrm{C}_{3} \mathrm{H}_{3} \mathrm{~N}(\mathrm{~S}=1)\right)$ & 27 & 30 & $3.5,4.0$ & $29 \pm 5$ \\
$\mathrm{C}_{3} \mathrm{H}_{3} \mathrm{~N}(\mathrm{~S}=0)$ & -3.0 & +4.7 & $-0.6,2.3,3.1$ & $-8 \pm 10$ \\
$\mathrm{C}_{2} \mathrm{H}_{2} \mathrm{~N}_{2}(\mathrm{~S}=0)$ & 39 & 42 & & $45 \pm 10$ \\
$\mathrm{C}_{2} \mathrm{H}_{2} \mathrm{~N}_{2}(\mathrm{~S}=1)$ & 56 & 60 & & $58 \pm 5$ \\
\hline azatetrahedrane & 26 & 22 & $1.4,4.7$ & \\
\hline dewar triazine & 52 & - & & \\
\hline
\end{tabular}

The valence isomers of pyrazine are energetically well-situated to interact with the excited singlet and triplet states of pyrazine. The more stringent energy requirements of the two dewar pyrazine isomers $\left(\mathrm{C}_{2} \mathrm{H}_{2}\right.$ and $\mathrm{N}_{2}$, where the designation refers to the species on the external edge of the bicycle) are reflected in their yields and wavelength dependence.

The geometry of the $\mathrm{S}_{0}\left({ }^{1} \mathrm{~A}_{1 \mathrm{~g}}\right)$ and $\mathrm{T}_{1}\left({ }^{3} \mathrm{~B}_{3 \mathrm{u}}\right)$ states of pyrazine are plotted to show the noticeable change in the $\mathrm{C}-\mathrm{N}$ and $\mathrm{C}-\mathrm{C}$ bond distances (see figures 1-54 and 155). The geometry is favorable for spin localization on the nitrogen which is supported 
by optical microwave double resonance experiments which measure a large value for $D$ (which measures the distortion of the spin distribution, see ref. 15) and is similar to structures predicted for the lowest triplet state of benzene $\left({ }^{3} B_{1 u}\right.$, see ref. 29$)$ The less energetically favorable resonance structures with the parallel spin electrons on para carbon atoms can provide a pathway to the prefulvene diradical. The first excited singlet state $\left(S_{1}\right)$ is predicted to be closer to the $S_{0}$ structure as in benzene (ref. 30 ).

The dewar pyrazine structure (see figure 1-57) shows notable distortion due to the presence of nitrogen, most importantly an elongation of the single bonds to the central bridging atoms. The decrease in the double bond distance of the $\mathrm{C}=\mathrm{N}$ group results from greater electron localization provides further geometric alteration that moves the species farther down the reaction coordinate to forming $\mathrm{HCN}$ plus the monoazete. This structure is probably the closest approximation to the 'transition state' of the reaction.

The nitrogen substituted cyclobutadienes have been the subject of previous calculations (ref. 26). These studies recognized the potential biradical nature of the azetes and calculated ground state configurations and excitations based upon complete active space self-consistent field calculations. Relatively small basis sets $(4-31 \mathrm{G})$ were used and the ground state geometry was constrained to be planar. The resulting ground state structure for the monoazete was of $C_{s}$ symmetry and a singlet, although $C_{2 v}$ geometry provided structures with energy $7-10 \mathrm{kcals}$ higher, and the nearest triplet state only $16 \mathrm{kcals}$ higher. Since the Hamiltonian and basis sets would give different reference energies, comparing the absolute energies of these configurations was not possible. 
We performed calculations based upon the G2 method and allowed freedom to break planar symmetry in attempts to determine these energies. The monoazete shows geometric properties similar to cyclobutadiene: a rectangular ground state singlet and a closer to square triplet state (see figures 1-59 and 1-62). The triplet geometry is a slightly non-planar trapezoid which matches well with the $\mathrm{x}$-ray diffraction structures found for substituted monoazetes complexed to cobalt (ref. 31 ). It is perhaps too early to conclude whether the singlet or the triplet is the ground state- similar calculations performed on cyclobutadiene predicted a ground state triplet until higher order configuration interaction and electron correlations were included. The fact that there are two very close lying states will lead to significant mixing and splitting so that the ground state will possess significant 'biradical character.' The work of Michl (ref. 26) also predicts significant distortion in the geometries of these azetes and thus facile interconversion and relatively large amplitude motions which could help induce chemical reactions via curve crossings. The rectangular form for the singlet is consistent with orbital overlap arguments concerning four membered ring systems (ref. 32).

Recent synthetic work with azetes has followed closely the methods used for cyclobutadienes. The thermodynamically stable compounds tris(dimethylamino)azacyclobutadiene (ref. 37) and 2-phenylbenzazete (ref. 38) have been prepared. As predicted by the orbital symmetry correlation methods described in the next chapter, these azetes are thermally robust. Tris(dimethylamino)azacyclobutadiene will show no change in its NMR spectrum even after days of heating to $100^{\circ} \mathrm{C}$. It undergoes cycloaddition reactions with conjugated 
dienes (ref. 38,39$)$ as well as a cycloreversion and fragmention following flash pyrolysis and photolysis (ref. 40). By introducing substituents at alternating ring position the azete is stabilized by the 'push-pull' effect (ref. 41) which donates or withdraws electron density in a manner favorable to the orbital energetics. This also can lead to distortion in the azete structure due to pronounced polar resonance forms (ref. 42).

Spectroscopic studies of substituted cyclobutadienes have shown that a dynamic equilibrium exists between the two valence isomers that could not be slowed on the NMR time scale by cooling to $-185^{\circ} \mathrm{C}$ (ref. 43). This facile intraconversion has been described as 'heavy atom tunneling' and has led to a theoretical search for path intermediates (ref. 36).

\section{Map of Azabenzene Photoreactivity}

From our experimental data and previous work we may construct a chart to visualize the photoreactive character of nitrogen substituted aromatic systems. Figures 1-64 and 1-65 show the branching ratio into various product channels at 248 and $193 \mathrm{~nm}$, respectively. The data for benzene is from reference 21 ; that for pyridine is from reference 22 supplemented by brief experimental work done at $248 \mathrm{~nm}$; for triazine reference 44 was supplemented with the data from section 4; finally, for tetrazine the work of Glownia et al. (ref. 45) was used. The reaction products are described by the lighter co-fragment, with the proviso that for the acetylene and propargyl channels a nitrogen may substitute for a $\mathrm{CH}$ moiety. 
Looking at the $248 \mathrm{~nm}$ map first, the most notable features are the large molecular hydrogen peak for benzene and the prominent $\mathrm{HCN}$ ridge appearing for the azabenzenes. The effect of the 'heteroatom', nitrogen, is pronounced very abruptly and with increasing dominance as the number of nitrogens present in the ring grows. Figure 1-66 rationalizes this dramatic shift based solely on energetics. According to our reaction model, the lowest energy benzene must correlate with the relatively high energy products cyclobutadiene plus acetylene. The other energetically closed channel, formation of two propargyl radicals, is likewise unfavorable. The presence of nitrogen destabilizes the reactants by about $1 / 2 \mathrm{eV}$ per nitrogen atom and stabilizes the products about $1 \mathrm{eV}$ per nitrogen (comparing acetylene to hydrogen cyanide). The reaction path involving $\mathrm{HCN}$ elimination becomes energetically favorable as well as the $\mathrm{N}$-substituted propargyl (methylene nitrile) channel and both are photochemically expressed. For benzene, these channels require additional energy such as a second photon to occur. The azabenzenes also show both atomic and molecular hydrogen elimination, although the latter can only occur significantly for mono and diazines.

Moving to $193 \mathrm{~nm}$ (figure 1-65) benzene's photoreactivity shifts strongly to atomic hydrogen elimination. Pyrazine and s-triazine follow this trend which reflects the predominance of simple bond rupture given enough excess energy. The behavior of stetrazine is inferred from $248 \mathrm{~nm}$ excitation. The relatively low pyridine $\mathrm{H}$ yield and high $\mathrm{C}_{3} \mathrm{H}_{3} / \mathrm{C}_{2} \mathrm{H}_{2} \mathrm{~N}$ and $\mathrm{HCN}$ yields do seem rather anomalous, as does the complete absence of molecular hydrogen elimination. The dramatic shift in the HCN translational energy release observed in triazine and somewhat in pyrazine is also not observed in pyridine. 
The diazines and triazines show additional complexity due to isomerization products which occur to a small extent.

\section{An Application: Reinterpreting the Phenomenon of Supercollisions}

A notable consequence of pyrazine's photochemical behavior is reconsideration of the mechanism of 'supercollisions.' Vibrational relaxation studies have measured rotational and translational energy transfer between $\mathrm{S}_{2}$ photoexcited pyrazine and $\mathrm{CO}_{2}$ bath molecules of up to $1 \mathrm{eV}$ per collision (ref. 46). Based upon spectroscopic information, it was inferred that hot ground state pyrazine was colliding with carbon dioxide and giving up this energy through intermolecular vibrational energy transfer. Our study of pyrazine's photoreaction dynamics suggests an alternate explanation: collisional energy transfer between $\mathrm{CO}_{2}$ and the translationally fast $\mathrm{HCN}$ photoproducts.

Describing intermolecular vibrational relaxation rates in relation to competing processes directing energy flow is fundamental to understanding chemical reactivity. Modern studies have progressed in detail far beyond determination of the pressure and temperature dependence of reaction rate coefficients (ref. 47). The amenable spectroscopic properties of pyrazine, namely its propensity to both fluoresce and phosphoresce, have made it a model system to study vibrational energy transfer following photo-excitation. Single vibronic level decay can be measured by monitoring fluorescence from the first excited singlet state (ref. 48) or phosphoresence from the first triplet state (ref. 49). Extending the range of available energy is challenged by the sharp 
drop off in fluorescence yield from the higher excited states and fast radiationless processes which occur (ref. 11). An ingenious method which recaptures the level of quantum state detail is to monitor the relaxation processes via interrogation of the bath molecules which accept energy from the more complex donor molecules (ref. 50). When the electronic relaxation mechanisms are well known (ref. 51), one can describe the energetically activated donor with confidence and focus experimental efforts on characterizing changes in the acceptor, allowing characterization of rotational, translational and vibrational energy transfer to the simpler bath molecules after single collision excitation (ref. 46). The energy transfer characteristics can then be modeled and described within the framework of collision geometries (ref. 48), angular momentum constraints and vibrational degrees of freedom (ref. 52).

As detailed and sensitive as the measurements of the bath molecules may be, they are also blind to the identity of the perturbation they seek to characterize. The influence of a 'reactive component' inducing the bath excitation has been an obvious concern that is handled as carefully as possible in such studies (ref. 53). Based upon the experimental results described earlier in this chapter, one can evaluate the possible effect of the reactive component in light of pyrazine's photodissociation following $248 \mathrm{~nm}$ excitation.

The azabenzenes, or nitrogen substituted benzene compounds, are known to liberate translationally fast $\mathrm{HCN}$ following photoexcitation (ref. 22, 44, 45). Previous photochemistry studies of the diazines have focussed primarily upon isomerization processes (ref. 54). Using photofragment translational spectroscopy we found a rich variety of products. The translational energy distributions and absolute 
photodissociation cross sections of the two dominant fast channels are presented in table 5 and figure 1-28. Concentrating upon the fast tail of the first channel (27-A) we consider the energetic limits of $\mathrm{HCN} / \mathrm{CO}_{2}$ collisions.

The collision between two closed-shell, relatively 'stiff' species with a mass ratio of 5:8 allows for very efficient translational $(\mathrm{T} \rightarrow \mathrm{T})$ energy transfer as well as possible vibrational excitation. Although such collisions will occur over the full range of possible orientations, impact parameters and relative velocities, a simplified view of two rotationally cold molecules $\left(\mathrm{HCN}\right.$ and $\left.\mathrm{CO}_{2}\right)$ colliding can provide an intuitive picture that can be easily understood. For small impact parameters $b$ (see figure 1-67) we expect translational energy transfer to dominate, with a maximum of $(5 / 8) \mathrm{E}_{\text {trans }}$ or a full $1 \mathrm{eV}$ transfer for 'head-on' $(\mathrm{b}=0)$ collisions, even slightly more allowing for the thermal velocity of $\mathrm{CO}_{2}$. Mullin et al. (ref. 46) measure translational energy changes up to 0.7 eV (relative velocity corrected). Broadside collisions can excite the $v_{2}$ bend while endon impacts are expected to cause a slight amount of $v_{3}$ (asymmetric stretch) motion with little concomitant rotational excitation. The $2349 \mathrm{~cm}^{-1}(0.3 \mathrm{eV})$ required for one quanta of $v_{3}$ excitation is energetically possible. Another significant excitation mechanism could involve vibrational to vibrational $(\mathrm{V} \rightarrow \mathrm{V})$ energy transfer from the vibrationally excited HCN liberated in the photodissociation process. The vibrational frequencies of $\mathrm{HCN}$, notably 2096 and $3312 \mathrm{~cm}^{-1}$, are reasonably well matched to the $\mathrm{CO}_{2}$ species.

As the impact parameter increases we can relate the relative velocity changes to angular momentum excitation by the equation 


$$
(h / 2 \pi) \Delta \mathbf{J}=\mu\left(\Delta \mathbf{v}_{\text {rel }}\right) \mathrm{b}
$$

where $\Delta \mathrm{J}$ is the change in angular momentum, $\mu$ is the reduced mass of $\mathrm{HCN} / \mathrm{CO}_{2}$ $=27 * 44 /(27+44) \mathrm{amu}, \Delta \mathrm{v}_{\mathrm{rel}}$ is the change following collision, and $\mathrm{b}$ the impact parameter. Large rotational excitation will follow collisions with large changes in relative velocity. We would expect the amount of angular momentum transfer to grow with increasing $b$ to a maximum product $\left(\Delta \mathbf{v}_{\text {rel }}\right.$.b) and then fall off as the collisions become more glancing and inefficient at transferring energy. We can estimate the amount of rotational excitation possible using a simple impulsive model (ref. 53) as up to $1 / 3$ of the excess (translational energy), or up to $0.5 \mathrm{eV}$ (see figure 1-68). Mullin et al. (ref. 46) measure up to $0.3 \mathrm{eV}$ $(\mathrm{J}=82)$. The 'breathing ellipsoid' model of Flynn (ref. 56) predicts that geometries slightly displaced from a direct end-on collision will contribute most to rotational excitation. The efficiency of energy transfer from $\mathrm{HCN}$ to $\mathrm{CO}_{2}$ by such a mechanism will be significant by virtue of the like masses of each particle.

Time dependent properties of a kinetic model due to collisions and diffusion will be similar for any collision partner (excited pyrazine, $\mathrm{HCN}$ ) formed from photoexcitation. The kinetic rate constants $\mathrm{K}^{\mathrm{J}} / \mathrm{Z}$ which fall into the range $1 / 30$ to $1 / 100$ (ref. 46) per collision match reasonably the quantum yields measured for $\mathrm{HCN}$ photoproduction and the contribution expected from the fast tail of $\mathrm{P}\left(\mathrm{E}_{\text {trans }}\right)$. The $\mathrm{HCN}$ photoproducts were ultimately detected at intensities of $0.2 \mathrm{MW} / \mathrm{cm}^{2}$ at $248 \mathrm{~nm}$ and 3 $\mathrm{MW} / \mathrm{cm}^{2}$ at $308 \mathrm{~nm}$ to show linear power dependence, roughly the field strengths associated with an unfocused excimer beam. 
A more direct method of evaluating the role of $\mathrm{HCN}$ would be $\mathrm{o}$ attempt to measure its translational and rotational energy directly. Based upon the wavelength dependent quantum yields one would also predict large energy transfer following $308 \mathrm{~nm}$ excitation to be drastically lower than $248 \mathrm{~nm}$, while if collisions with hot ground state pyrazine dominate the $20 \%$ reduction in internal energy should have a more modest effect. Photodissociation of 'donors' such as tetrazine and triazine are expected to show similar excitation characteristics to the $\mathrm{HCN}$ produced from pyrazine.

In conclusion, the photoreactive behavior of pyrazine at 248 and $308 \mathrm{~nm}$ exhibits a dramatic change which may have importance in the interpretation of collisional relaxation studies. The quantum yields and translational energy release of the photoproduction of HCN has been shown to be a potential mechanism for the phenomenon of 'supercollisions' involving pyrazine. 


\section{H. References}

1. F. A. Cotton, Chemical Applications of Group Theory, Wiley and Sons, New York, 1974.

2. R. S. Mulliken and R. G. Parr, J. Chem. Phys.,19, 1271 (1951).

3. R. S. Mulliken, C. A. Rieke and W. G. Brown, J. Am. Chem. Soc.,63, 41 (1941).

4. JANAF Thermochemical Tables, 2nd and 3rd eds.; also J. Phys. Chem. Ref. Data, Vol. 14, suppl. (1985).

5. A. Streitweiser and C. Heathcock, Introduction to Organic Chemistry, MacMillan, New York, 1981.

6. A.M. Wodtke and Y.T. Lee, J. Phys. Chem., 1985, 89, 4744.

7. D.R. Miller, "Free Jet Sources," in Atomic and Molecular Beam Techniques, G.

Scoles, ed. Oxford University Press, 1979.

8. Atlas of Mass Spectral Data, Vol 1. E. Stenhagen, S. Abrahamssom and F.W. McLafferty, eds. John Wiley and Sons, 1961.

9. C. Fridh, L. Asbrink, B. Jonsson and E Lindholm, Int. J. Mass Spectrom. Ion Phys., 8 (1972), 101-118; ibid. 8 (1972), 85-99; ibid 8 (1972), 215-236.

10. A. Bolivinos, P. Tsekeris, J. Philis, E. Pantos and G. Andritsopolous, J. Mol. Spectroscopy, 103, 240-256 (1984).

11. I. Yamazaki et al., Faraday Diss. Chem. Soc., 1983,75, 395-405 (1984).

12. W.A. Noyes and K.E. Al-Ani, Chem Rev., 1974,74, 29.

13a. W. Roebke, J. Phys. Chem., 1970, 74, 4198. 
13b. F. Lahmani and N. Ivanoff, Tetrahedron Letters, 1967, 3913 and J. Phys. Chem., $1972,76,2245$.

14. S.K. Lower and M.A. El-Sayed, Chem. Rev. 66, 199 (1966).

15. K.K. Innes, I.G. Ross and W.R. Moomaw, J. Mol. Spectroscopy, 132, 492-544 (1988).

16. H. Leidreiter, H. Wagner, Z. Phys. Chem. N.F., 153, 99 (1987).

17. G. Herzberg, Spectra of Diatomic Molecules, Van Nostrand Reinhold, New York, 1950.

18. P.W. Harland, Int. Journal Mass Spec. Ion Processes, 70, 231 (1986).

19. S.K. Pollack and W. J. Hehre, Tetrahedron Letters, 21, 2483 (1980).

20. L. Radon, R. Nobes, D. Underwood and W. Li, Pure and Appl. Chem., Vol. 58, No. $1, \mathrm{p} 75-86$.

21. A. Yokoyama, X. Zhao, E. Hintsa, R. Continetti and Y.T. Lee, J. Chem. Phys., 92 (7), 4222 .

22. K.A. Prather and Y.T. Lee, Israel Journal of Chemistry, Vol. 34, 43-53 (1994).

23. M. Klessinger and J. Michl, Lichtabsorption und Photochemie Organischer Molekule, VCH, Berlin, 1989.

24. G.S. Ondrey and R. Bersohn, J. Chem. Phys., 81(10), 4517 (1984).

25. B. Atless, L. Schaad, C. Holyoke, Tetrahedron 31, 295 (1975) and J. Org. Chem, 41, $12(1976)$.

26. V. Bonacic-Koutecky, K. Schuffett and J. Michl, J. Am. Chem. Soc.,111, 1988 (1989). 
27. L.A. Curtis, K.R. Raghavachari, G.W. Trucks and J.A. Pople, J. Chem. Phys., 94(11), 7221 (1994).

28. T. Clark, "A Handbook of Computational Chemistry," John Wiley and Sons, New York 1985.

29. A.D. Liehr, A. Naturforsch., 16a, 64 (1961).

30. L. Salem, "The Molecular Orbital Theory of Conjugated Systems," W.A. Benjamin and Co., New York, 1966.

31. M. Ledermann et al., Angew. Chem., 100, 1616 (1988).

32. H.C. Longuet-Higgins, "Theoretical Organic Chemistry," Kekule Symposium, Butterworth, London, 1959.

33. R.E. Center and A. Mand1, J. Chem. Phys., 1972,57, 4104.

34. K. Bystrom, J. Chem. Thermodyn, 14, 865 (1982).

35. E.E. Koch and A. Otto, Int. S. Radiat. Phys. Chem., 8, 113 (1976).

36. M. Gluhovchev, B. Simkin, V. Minkin, Zhurnal Org. Khim., 19(7), 1353 (1983).

37. G. Seybold, U. Jersak, R. Gompper, Angew. Chem. Int. Ed. Engl., 12 (1973), 847.

38. B.M. Adger, C.W. Rees, R.C. Storr, J. Chem. Soc. Perkin Trans., 1 (1975), 45.

39. M. Regitz, Nachr. Chem. Tech. Lab., 39 (1991), 9.

40. U. Vogelbacher, M. Regitz, R. Mynott, Angew. Chem. Int. Ed. Engl., 25 (1986), 842.

41. J.D. Roberts, J. Chem. Soc. Spec. Publ. No. 12, 111 (1958).

42. H. Wagner, Angew. Chem. Int. Ed. Engl., 12 (1973), 848.

43. G. Maier, H. Kalinowski, K. Euler, Angew. Chem. Int. Ed. Engl., 21 (1982), 693. 
44. G.S. Ondrey and R. Bersohn, J.Chem. Phys., 81, 4519 (1984).

45. J.H. Glownia and S.J. Riley, Chem. Phys. Lett., 71, 429 (1980).

46. A.S. Mullin et al., Chemical Physics, 175 (1993), 53.

47. I. Oref and D. Tardy, Chem. Rev., 90, 1407 (1990).

48. D.B. MacDonald and S.A. Rice, J. Chem. Phys., 74(9), 4907.

49. T.A. Bevilacqua and R.B. Weisman, J. Chem. Phys., 98(8), 6316.

50. J.O. Chu et al., J. Chem. Phys., 81(12), 5533.

51. A.E.W. Knight and C.S. Parmenter, Chemical Physics, 15(1976), 85;

A. Frad, F. Lahmani, A. Tramer and C. Tric, J. Chem. Phys., 60(1974), 4419;

D.B. MacDonald and S.A. Rice, Chemical Physics, 60(1981), 335;

T.G. Dietz et al., J. Phys. Chem., 86(1982), 4026;

P.A.M. Uijt de Haag et al, Chemical Physics, 135 (1989), 139.

J. Knee and P. Johnson, J. Phys. Chem., 89(1985), 948.

52. J.Z. Chou and G.W. Flynn, J. Chem. Phys., 93(8), 6099.

J.A. O’Neill et al., J. Chem. Phys., 88(10), 6240.

53. A.J. Sedlack et al., J. Chem. Phys., 94(10), 6483;

J.M. Morgulis et al., J. Chem. Phys., 90(2), 923.

54. A. Lablanche-Combier, in "Photochemistry of Hetercyclic Compounds," O.

Butchardt, Ed. Vol. 4, pp. 208-215.

55. G.E. Busch and K.R. Wilson, J. Chem. Phys., 56, 3626 (1972).

56. G.W. Flynn and R.E. Weston, Jr., J. Phys. Chem., 97, 8116 (1990). 


\section{Figure Captions}

Figure 1-1. A schematic representation of the rotating source machine. The experimental sequence proceeds as follows. A gas sample is expanded into the source region 2 from a pulsed valve assembly 1 , where it is skimmed twice as it passes through a differential pumping region 3 , and travels into the main chamber 4 . A laser beam is pulsed to collide with the molecular beam in the interaction region 5 and recoiling fragments pass through a defining aperture 6 if their resultant velocity vector, dependent upon the source to detector angle a, so permits. The neutral fragments are ionized through electron impact at 7 , collimated at 8 and quadrupole mass filtered at 9 . Ions which are transmitted then are accelerated to the Daly doorknob 10 which is at $-30 \mathrm{kV}$, inducing a shower of electrons which impinge upon the scintillator and are detected by the photomultiplier tube 11 , and recorded by a multichannel scalar.

\section{Figure 1-2.}

An example of how application of equation (8) can account for both constructive and destructive interference in the signal seen near the beam velocity. The top trace $(\mathrm{m} / \mathrm{e}=52)$ shows both a fast peak near 160 microseconds and a null, the superposition of a signal and a hole, near 240 microseconds. The 'hole' can be seen in the second trace, representing the depletion of the parent $(\mathrm{m} / \mathrm{e}=79)$ following photoexcitation. The lowest trace has background reduced dramatically due to acquisition at a slightly larger 
angle and different $\mathrm{m} / \mathrm{e}(=51)$. The smaller peak near 250 microseconds energetically matches the fraction of a peak only partially seen in the upper trace.

Figure 1-3.

The method of determining absolute photoreactive cross sections involves comparing the area of lower trace ('hole', or depleted parent) with the middle trace (undepleted parent) over the time interval defined by the laser in the interaction region and subsequent spreading of the pulse. From this ratio of areas the fraction of reacting molecules can be determined. If the laser beam intensity and shape is measured and absolute photodissociation cross section can be calculated. Determination of absolute quantum yields requires knowledge of the absorption cross section, which is generally well known for continuum absorptions in the ultraviolet.

Figure 1-4.

The ionization and fragmentation scheme used for interpreting the time of flight spectra for different $\mathrm{m} / \mathrm{e}$ ions. The predominance of both (sequential) $\mathrm{H}$ and $\mathrm{HCN}$ loss from the mechanism described in reflected in the fragmentation patterns described below. The energy dependence of each of these processes was checked in support or proposed mechanism. Of importance as well is the small yield of fragmentation to $\mathrm{m} / \mathrm{e}=27$ (esp. $\mathrm{HCN}+$ ), which is consistent with the mass spectrometric rule concerning which species will bear the charge following cation fragmentation. 
Figure 1-5.

The absorption spectra of pyrazine, taken from reference 10 .

Figure 1-6.

The excitation profiles of excimer pulses, as provided by Lambda Physik.

Figure 1-7.

Calculated RRKM lifetimes for a reaction barrier $90 \mathrm{kcals}$ above the zero point energy of pyrazine's ground state minimum. Both the total energy and the perceived barrier height can dramatically influence the dissociation lifetime. For energies near threshold, the relatively large number of vibrations in pyrazine (24, taken from ref. 15$)$ can slow down 'ground state' reaction to well beyond even the flight time of our experimental measurement.

Figure 1-8.

Neutral fragment time-of-flight spectra of Pyrazine following $308 \mathrm{~nm}$ excitation. Actual count rates are plotted on the ordinate to allow meaningful signal to noise estimates for different regions of the spectra. The $m / e=53$ trace shows two faint peaks, the faster one which is shown more clearly in the $\mathrm{m} / \mathrm{e}=52$ trace and corresponding to channel $27-\mathrm{A}$, elimination of $\mathrm{HCN}$ to probably leave the monoazete. Whether the monoazete persists or rearranges to a stable form such as acrylonitrile is unclear. The differing intensity ratio observed in the $\mathrm{m} / \mathrm{e}=53$ and $\mathrm{m} / \mathrm{e}=52$ signals, coupled with the small $\mathrm{m} / \mathrm{e}=27$ signal 
peaked near zero energy indicate that the slower peak (channel 53-A) may be simply from photosensitized ion fragmentation.

Figure 1-9.

Neutral time of flight spectra of the lighter fragments $\mathrm{m} / \mathrm{e}=27(\mathrm{HCN}), \mathrm{m} / \mathrm{e}=26$ ( mostly acetylene $\left.\mathrm{C}_{2} \mathrm{H}_{2}\right)$ and $\mathrm{m} / \mathrm{e}=25\left(\mathrm{C}_{2} \mathrm{H}+\right)$ show the presence of two acetylene elimination channels from two photon processes. The slower two $\mathrm{m} / \mathrm{e}=26$ peaks are from fragmentation of the $m / e=53$ channels. A similar (translationally fast) two photon peak for $\mathrm{HCN}$ is observed without the matching $\mathrm{m} / \mathrm{e}=53$ fragment due to the latter's subsequent dissociation. The large, central peak fit at $\mathrm{m} / \mathrm{e}=27$ is from channel $27-\mathrm{A}$ and matches the fast $\mathrm{m} / \mathrm{e}=52$ and 53 fragments. The slow shoulder at $\mathrm{m} / \mathrm{e}=27$ matches the slower of the two acetylene channels. The $\mathrm{m} / \mathrm{e}=25 \mathrm{scan}$ is done to confirm the extent of $\mathrm{m} / \mathrm{e}=27$ interference.

Figure 1-10.

Neutral time-of-flight spectrum of $\mathrm{m} / \mathrm{e}=26$ following $308 \mathrm{~nm}$ excitation. The higher angle scans (20 and 30 degrees) are able to resolve the two contributions to the faster side of the trace.

Figure 1-11.

The probability of translational energy release for the two one-photon channels observed following $308 \mathrm{~nm}$ excitation of pyrazine. The $\mathrm{HCN}$ elimination channel, 27-A, was 
taken from the fit of the $\mathrm{m} / \mathrm{e}=52$ fragment to minimize the interference from multiphoton processes. The second channel, 53-A, is peaked close enough to zero that the energy width could simply be caused by the beam velocity drifting a few percent over the long duration of the scan, and is tentatively assigned to photon enhanced ion fragmentation.

Figure 1-12.

The probability of translational energy release of acetylene following multiphoton $308 \mathrm{~nm}$ excitation. Two channels are described based upon the fit of the $m / e=25$ fragments. Because the yields were so small (and signal to noise correspondingly small) there is considerable uncertainty in the details of both distributions.

Figure 1-13.

Power dependence of the dominant channels following $308 \mathrm{~nm}$ and $193 \mathrm{~nm}$ excitation. For $308 \mathrm{~nm}$, channel 27-A and 53-A show a flat response of counts given a constant level of excitation times scans, indicative of linear power dependence. Channel 26-A/B (summed, and not corrected for HCN fragmentation) shows an increase that is not quite quadratic, but still strongly dependent on laser intensity and thus likely to be multiphoton in origin.

For the $193 \mathrm{~nm}$ graph, the $\mathrm{m} / \mathrm{e}=27$ (HCN elimination channels) were checked at very low fluences by monitoring the $\mathrm{m} / \mathrm{e}=52$ peak and found to be basically flat. The sharper decline seen in channels $27-\mathrm{C}$ and 27-D are from depletion of these channels following stronger excitation, which creates fast fragments whose heavier partners (at $\mathrm{m} / \mathrm{e}=53,54$ ) 
do not survive. The $\mathrm{m} / \mathrm{e}=26$ line was determined from the corrected contribution of $\mathrm{m} / \mathrm{e}=27$ to this signal, as noted from the intensity of the $\mathrm{m} / \mathrm{e}=53$ peak which fragments efficiently to $26+$. The flat behavior and non-zero contribution at vanishly low intensities imply this minor channel is accessible after single photon excitation.

Figure 1.14

The power dependence of major channels seen following $248 \mathrm{~nm}$ excitation. Note that relatively large excitation probabilities were studied to also monitor the changes in energy release, especially into the translationally fast channels. The HCN elimination paths $27-\mathrm{A} / \mathrm{B}$ and $27-\mathrm{C}$ show linear behavior at low intensities but then saturate, while actylene elimination grows rapidly as intensity increases (indicating multiphoton dependence) and then also saturate at a level comparable to the 27 signal. The methylene nitrile channel shows linear dependence over the limited range studied. The power dependence of the proposed nitrogen elimination channel was not studied because of very low signal to noise and the inability to procure the isotopic sample which would allow more accurate determination.

Figure 1-15.

$\mathrm{H}$ atom elimination from pyrazine following $248 \mathrm{~nm}$ excitation. Because the $\mathrm{m} / \mathrm{e}=1$ peak could be fit energetically by considering solely $\mathrm{H}$ fragmentation, a long scan at $\mathrm{m} / \mathrm{e}=79$ was performed. The low signal to noise of this latter scan is strong evidence of subsequent fragmentation of the $\mathrm{m} / \mathrm{e}=79$ pyrazyl following ionization. The energetic 
parameters for the channel were determined from fitting the fast peak of the light $\mathrm{H}$ fragment. The second, broader envelope was fit with the fast HCN elimination channel 27-A. The slow tail of the signal suffered broadening from fragments that would collide before scattering into the detector, since the beam source was unskimmed and only a centimetre from the valve opening.

Figure 1-16.

Hydrogen (molecular deuterium) loss following $248 \mathrm{~nm}$ excitation shows distinctive shoulders that match the energy spacing of deuterium's vibrational frequency. The $\mathrm{m} / \mathrm{e}=78$ trace shows an energy width able to fit roughly two quanta of hydrogen vibration, and an additional contribution at the beam velocity likely due to pyrazyl fragmentation.

Figure 1-17.

Neutral fragment time-of-flight of $\mathrm{m} / \mathrm{e}=53$ ion following $248 \mathrm{~nm}$ excitation. Of importance is the shoulder observed on the fast peak, corresponding to channel 27-B, most readily seen at a detector angle of 15 degrees.

Figure 1-18.

Time-of-flight spectrum of the $\mathrm{m} / \mathrm{e}=52$ fragment which results from $\mathrm{m} / \mathrm{e}=53$ cracking following ionization. Being able to match the tail of the first peak required a second contribution (channel 27-B) of different weighting than channel 27-A, as the fit should 
demonstrate. Resolving the three contributions (channels 27-A, 27-B and 27-C) is achieved by the self-consistent fitting procedure (program CMLAB2) applied over a wide range of detector angles.

Figure 1-19.

The time-of-flight at $\mathrm{m} / \mathrm{e}=40$ following $248 \mathrm{~nm}$ excitation of pyrazine. Due to the low background at this mass, a very high resolution trace was obtained at small angles and demonstrated the existence of two components. The slower peak, channel 40-B could conceivably arise from fragmentation of higher mass fragments such as the pyrazyl radical.

Figure 1-20.

The time-of-flight of HCN following $248 \mathrm{~nm}$ excitation of pyrazine. To corroborate the three signals ascribed to $\mathrm{m} / \mathrm{e}=53$ and 52 fragments, these peaks were momentum matched or fit with the same energy distributions (i.e. 27-A, 27-B, 27-C). There is a contribution to $\mathrm{m} / \mathrm{e}=53$ cracking to $27+$ underneath channel $27-\mathrm{B}$ that is consistent with the fragmentation model and measured intensities of channels 27-A and 27-B.

Figure 1-21.

The time-of-flight spectra of HCN at higher detector angles. Only channels 27-A and 27-B appear in these traces based upon kinematic constraints. 
Figure 1-22

Evidence for the matching fragment following acetylene loss was tenuous due to the poor survivability of $m / e=54$. As suggested by the triazine mass spectrum, this fragment could be seen best at low electron impact energies. This scan was taken with $30 \mathrm{eV}$ bombardment electrons and suggests two peaks which, upon careful inspection, are slightly slower than $\mathrm{m} / \mathrm{e}=53$ fragments. The large background signal coming later than 200 microseconds is from arrival of the pulse moving at the beam velocity; that the peaks come earlier than this point is why they can be resolved. The lower trace shows the $\mathrm{m} / \mathrm{e}=25$ trace fit with the same energy distributions and weightings, with a large contribution from the $\mathrm{m} / \mathrm{e}=53$ fragment cracking to $\mathrm{m} / \mathrm{e}=2 \dot{6}$ and then $25+$.

Figure 1-23.

The evidence for dinitrogen elimination following $248 \mathrm{~nm}$ excitation of pyrazine. The upper trace shows the $m / e=49$ fragment, which can only come from $m / e=52\left(\mathrm{CH}_{4}\right)$ cracking through sequential hydrogen loss. Again, only the first (fast) peak is resolvable due to the beam background which grows in after 200 microseconds. The energy distribution used to fit this peak matches well with the spectrum observed at $m / e=28$, which suffers from high background contributions. The last (slowest) peak appears at the beam velocity and could be a number of precursors cracking, while the middle peak could be a second nitrogen channel. Note that the $m / e=28$ signal is much slower than the dominant HCN fragment. 
Figure 1-24.

The probability of translational energy released used to fit the molecular deuterium timeof-flight. The channels 2-B, 2-C and 2-D differ by about $8.5 \mathrm{kcal}$, and presumably match integral number of vibrational quanta present in the recoiling deuterium.

Figure 1-25.

The two probabilities of translational energy release used to fit the $\mathrm{m} / \mathrm{e}=40$ (methylene nitrile) signal. The second channel, 40-B, could conceivably be due to dissociative ionization of precursors. Because no such signal was present following $308 \mathrm{~nm}$ excitation, the chance that this is simply photon enhanced parent fragmentation is considered small.

Figure 1-26.

Probabilities of translational energy release $P(E)$ used to fit two photon product channels for pyrazine following $248 \mathrm{~nm}$ excitation- acetylene and nitrogen elimination. The second acetylene channel, 26-B, is very uncertain due to overlap with channel $27-$ A's $m / e=53$ fragment. These channels are both relatively minor.

Figure 1-27.

$P(E)$ used to fit the hydrogen loss channels (1-A) for pyrazine following $248 \mathrm{~nm}$ and $193 \mathrm{~nm}$ excitation. The energetic tails can suffer from two photon contributions and thus are relatively uncertain. 
Figure 1-28.

$P(E)$ 's used to fit the dominant chemical reactions of pyrazine following $248 \mathrm{~nm}$ excitation, channels 27-A, 27-B and 27-C. Because the products overlap in energy and thus also in the time-of-flight, the energetic limit of 27-B (about $18 \mathrm{kcals}$ ) is quite uncertain. The fast tail of 27-A may have a small contribution from two photon processes and also shares considerable uncertainty as well.

Figure 1-29.

The time-of-flight and $\mathrm{P}(\mathrm{E})$ for methyl loss from pyrimidine (1,3 diazine) following $248 \mathrm{~nm}$ excitation. This channel was relatively minor in the case of pyrimidine, but not observable in the case of pyrazine. This observation suggests that the ring opening pathway is separate from the isomerization process, and subsequent rearrangement of the carbons and nitrogen backbone is topologically constrained.

Figure 1-30.

Two traces of pyrazine fragmentation into $\mathrm{m} / \mathrm{e}=53$ following $248 \mathrm{~nm}$ excitation at two intensities provide further evidence of the two distinct $\mathrm{HCN}$ pathways 27-A and 27-B. In the high power (top) trace the $\mathrm{m} / \mathrm{e}=53$ co-fragment to $27-\mathrm{A}$ has completely disappeared and only 27-B's co-fragment can be seen. Depletion in channel 27-C is evidenced as well, as was observed following intense 193nm excitation, suggesting that a 
direct ring opening (simple bond rupture) may not be the preferred pathway to a linear intermediate.

Figure 1-31

The dramatic effect of varying the laser power on the product fragment distribution can be seen in these three traces taken at $\mathrm{m} / \mathrm{e}=26$ following $193 \mathrm{~nm}$ excitation. The azete fragments which appear as the large peak around 300 microseconds can be completely eliminated, and faster $\mathrm{m} / \mathrm{e}=26$ (and $\mathrm{m} / \mathrm{e}=27$ ) species generated in the process.

Figure 1-32.

Time-of-flight spectrum of hydrogen $(\mathrm{m} / \mathrm{e}=1)$ following $193 \mathrm{~nm}$ excitation of pyrazine and triazine. By comparison with the $248 \mathrm{~nm}$ spectrum (figure 1-10), we note an elevated 'baseline' that cannot be fit well with the $\mathrm{m} / \mathrm{e}=27$ contribution, and is therefore designated channel 1-B for pyrazine. The triazine spectrum was taken at a slightly higher power level because of time constraints and was meant to test what had been observed at $248 \mathrm{~nm}$, the presence of atomic hydrogen elimination (a channel not previously mentioned in earlier studies, but suggested by the pyrazine 'azabenzene' reactive model.)

Figure 1-33.

Time-of-flight of molecular deuterium elimination from pyrazine following $193 \mathrm{~nm}$ excitation. The signal levels were too weak to resolve more than just a large peak and a 
shoulder, but the basis functions from $248 \mathrm{~nm}$ were used to fit three components. The spectrum at $\mathrm{m} / \mathrm{e}=78$ again shows only an energy width to hold one quanta of hydrogen vibration, with considerable contribution from $\mathrm{m} / \mathrm{e}=79$ cracking.

Figure 1-34.

Time-of-flight spectra of pyrazine following $193 \mathrm{~nm}$ excitation at $\mathrm{m} / \mathrm{e}=53$ and 52 . Note that the two fastest peaks, corresponding to channels $27-\mathrm{A}$ and $27-\mathrm{B}$, have completely disappeared from the spectra, consistent with the energy dependence implied in our fragmentation model for the azetes. The $\mathrm{m} / \mathrm{e}=52$ peak provides the co-fragment to match these channels.

Figure 1-35.

Time-of-flight spectra of pyrazine following $193 \mathrm{~nm}$ excitation at $\mathrm{m} / \mathrm{e}=52$, at low angles. An additional slow channel, 27-D, was required to fit the spectra well at 5 and 10 degrees.

Figure 1-36

Time-of-flight spectra of pyrazine, $\mathrm{m} / \mathrm{e}=52$, following $193 \mathrm{~nm}$ excitation. At 15 and 25 degrees the two fastest channels, 27-A and 27-B, are best resolved.

Figure 1-37. 
Time of flight spectra of pyrazine, $\mathrm{m} / \mathrm{e}=27$, following $193 \mathrm{~nm}$ excitation. The slowest channels, 27-C and 27-D are best resolved at these low angles.

Figure 1-38.

Time-of-flight spectra of pyrazine, $\mathrm{m} / \mathrm{e}=27$, following $193 \mathrm{~nm}$ excitation. The fast channels, 27-A and 27-B are distinct at these detector angles.

Figure 1-39.

Time-of-flight spectra for pyrazine following $193 \mathrm{~nm}$ excitation at $\mathrm{m} / \mathrm{e}=26$. At low angles (10 degrees) the dominant signal is from slow fragments (cf. $\mathrm{m} / \mathrm{e}=53$, figure 1-29, channel 27-C) cracking. At higher angles the signal level drops considerably and the faster components due to $\mathrm{m} / \mathrm{e}=53$ (channels $27-\mathrm{A}, \mathrm{B}, \mathrm{D}$ ) become easily visible. The fastest two components are combined in this trace because of memory requirements of the computer program.

Figure 1-40.

Time-of-flight of pyrazine's $\mathrm{m} / \mathrm{e}=26$ fragment following $193 \mathrm{~nm}$ excitation. The fastest two components are the contributions from $26-\mathrm{A}$ and $26-\mathrm{B}$, while the largest and slowest component is from $\mathrm{m} / \mathrm{e}=53$, channel 27 -A cracking.

Figure 1-41. 
Time-of-flight of pyrazine following $193 \mathrm{~nm}$ excitation, at twenty degrees detector angle. The channels $26-\mathrm{A}$ and $26-\mathrm{B}$ are elucidated by comparison to the lower $\mathrm{m} / \mathrm{e}=25 \mathrm{scan}$, which was taken at medium power and thus shows a greater contribution in the fastest peak (cf. figure 1-26).

Figure 1-42.

Time-of-flight for pyrazine following $193 \mathrm{~nm}$ excitation, $\mathrm{m} / \mathrm{e}=40$ (methylene nitrile). Following the signal over the range 5-20 degrees with respect to the detector helped resolve three distinct channels, 40-A, 40-B and 40-C.

Figure 1-43.

$P(E)$ for molecular hydrogen loss following $193 \mathrm{~nm}$ excitation of pyrazine, channels 2-A, 2-B and 2-C.

Figure 1-44.

$\mathrm{P}(\mathrm{E}) \mathrm{s}$ for elimination of $\mathrm{CH}_{2} \mathrm{CN}$ (methylene nitrile), channels $40-\mathrm{A}, 40-\mathrm{B}$ and $40-\mathrm{C}$.

Figure 1-45.

$\mathrm{P}(\mathrm{E}) \mathrm{s}$ for the dominant $\mathrm{HCN}$ release channels following $193 \mathrm{~nm}$ excitation of pyrazine, 27-A and 27-B.

Figure 1-46. 
$\mathrm{P}(\mathrm{E}) \mathrm{s}$ for the two slow channels of $\mathrm{HCN}$ release following $193 \mathrm{~nm}$ excitation of pyrazine, 27-C and 27-D.

Figure 1-47

An energy level diagram for the species formed following photoexcitation of pyrazine. The heats of formation are quoted in kcals/mol relative to the standard state and uncertainties in the values discussed in the text. The energetics of intermediates and common closed shell species are not described.

Figure 1-48.

Time-of-flight of $1,3,5$ triazine at $\mathrm{m} / \mathrm{e}=1(\mathrm{H}$ loss) following $248 \mathrm{~nm}$ excitation. The fast peak is $\mathrm{H}$ atom loss while the slower envelope is from $\mathrm{HCN}$ fragmenting. The corresponding $\mathrm{P}(\mathrm{E})$ used to fit the data is presented below. The similarities to pyrazine are quite stunning.

Figure 1-49.

Time-of-flight of triazine at $\mathrm{m} / \mathrm{e}=27$ following $248 \mathrm{~nm}$ excitation. The dominant channel is loss of fast HCN but a slow tail to the peak can be discerned. The second minor peak is from a $m / e=54$ fragment matched to the fast 27 peak. The small size of this peak is consistent with mass spectral studies done on triazine fragmentation. The third even smaller peak is from fragmentation of a $\mathrm{m} / \mathrm{e}=53$ species generated from an isomerization process. 
The $\mathrm{m} / \mathrm{e}=26$ spectrum shows an even larger slow tail, consistent with our understanding of the fragmentation of the heavier azete cations.

Figure 1-50.

Time-of-flight spectrum of triazine following $248 \mathrm{~nm}$ excitation at $\mathrm{m} / \mathrm{e}=51$ and 40 . The former product is from isomerization followed by nitrogen elimination while the latter is from both rearrangement and fragmentation to methylene nitrile and secondary dissociation of the triazyl radical.

Figure 1-51.

Time-of-flight spectrum of triazine following $248 \mathrm{~nm}$ excitation at $\mathrm{m} / \mathrm{e}=54$. The ionizer was set at $30 \mathrm{eV}$ electron energy and the fast, slow trace magnified in the lower graph. The position of this peak is too fast for the $\mathrm{m} / \mathrm{e}=53$ peak detected at $\mathrm{m} / \mathrm{e}=51$ and the energy distribution is the one corresponding to fast HCN (channel 27-A).

Figure 1-52.

The probability of translational energy released for triazine's dominant $\mathrm{HCN}$ elimination channel (27-A, 248nm ) and the slow HCN channel 27-B thought to originate following conversion to the ground state and a linear form. According to our azabenzene reaction model, this latter channel (27-B) will become much more pronounced following $193 \mathrm{~nm}$ excitation, as is observed in experiment. 
Figure 1-53.

$P(E)$ 's for the $\mathrm{m} / \mathrm{e}=40$ elimination channels (both $40-A$ and $40-B$ ). The latter channel may result from dissociative ionization of the parent or triazyl radical, and is a relatively minor contribution to the overall reaction yield.

Figure 1-54

A plot of the geometry of pyrazine in the $\mathrm{S}_{0}\left({ }^{1} \mathrm{~A}_{1 \mathrm{~g}}\right)$ state, determined by $a b$ initio optimization at the RHF level with a $6-311+G^{*}$ basis. The molecule is planar and of $\mathrm{D}_{2 \mathrm{~h}}$ symmetry, but the shortened $\mathrm{C}-\mathrm{N}$ bond distances are indicative of the geometric changes resulting from substituting an electronegative heteroatom into the aromatic ring. The bond lengths are angles are very close to the structure determined by $\mathrm{x}$-ray diffraction.

Figure 1-55.

MP2/6-311+G* optimized geometry of the lowest triplet state $T_{1}\left({ }^{3} B_{3 u}\right)$. The shortened C-C bond lengths, elongated $\mathrm{C}-\mathrm{N}$ bonds and increase $\mathrm{N}-\mathrm{C}-\mathrm{N}$ angle is consistent with the spin localization observed on the nitrogen by optical-microwave double resonance experiments. Less energetically favorable resonance forms may be important diradical intermediates to the valence isomers benzvalene and fulvene.

Figure 1-56. 
The diaza-dewar pyrazine structure optimized at $R H F / 6-311+G^{*}$ levels. The notable distortion in the fused four-membered rings may help induce $\mathrm{HCN}$ elimination through cycloreversion.

Figure 1-57.

The diaza-benzvalene involved in pyrazine $\leftrightarrow$ pyrimidine isomerization. The structure was optimized at the $\mathrm{RHF} / 6-311+\mathrm{G}^{*}$ level. At higher energies a possible additional $\mathrm{HCN}$ loss mechanism could involve a $\left[\sigma_{s}+{ }_{\sigma}{ }_{s}\right]$ rearrangement to the tetrahedrane plus $\mathrm{HCN}$.

Figure 1-58.

The ground singlet state structure of pyrazine's fulvene isomer. This molecule is less than an eV higher in energy than pyrazine and possesses low lying triplet states that may provide a facile pathway to an open chain form. The mechanism of rearrangement to the fulvene geometry is thought to involve a 1,4 biradical (pre-fulvene) precursor.

Figure 1-59.

The triplet state monoazete calculated at the MP2/6-311+G* level. The symmetry is $\mathrm{C}_{1}$ as the molecule is slightly non-planar. Low energy triplet product channels will be important if the relaxation path involves triplet intermediates. 
Figure 1-60.

The lowest energy singlet monoazete calculated at the MP2/6-311+G* level, which is about an electron volt lower in energy than the triplet. The rectangular geometry is similar to the (ground) singlet state of cycobutadiene.

Figure 1-61.

The azatetrahedrene structure which could possibly be formed through a cycloreversion of the benzvalene intermediate. The steric constraints of the fused rings would not be favorable to this process, nor do the energetics of the tetrahedrane compensate well for such a mechanism.

Figure 1-62

The ground state structure of cyclobutadiene, which is a singlet at the MP2/6-311+G* level. The rectangular $D_{2 h}$.geometry is thought to rapidly interconvert between its two valence isomer forms through heavy atom tunneling.

Figure 1-63

The triplet (A) state structure of cyclobutadiene, calculated at the MP2/6-311+G* level. The almost square geometry can be compared to noticeable distortion present in the corresponding triplet monoazete.

Figure 1-64. 
A photoreactive profile of azabenzenes following $248 \mathrm{~nm}$ excitation. The reaction channels simply identify the product whose co-fragment must be characterized from experimental details. The molecules described range from benzene to tetrazine, with data taken from this thesis as well as refs. $21,22,24$ and 45 . The contours are projections of reaction probability graded at intervals of 0.10 and based upon experimental branching ratios.

Figure 1-65.

A photoreactive profile for azabenzenes following $193 \mathrm{~nm}$ excitation, with the same references used in 1-63 and data 'extrapolated' in the case of tetrazine. Although the extremes of benzene and tetrazine exhibit very sharply peaked branching ratios, other azabenzenes show intermediate behavior across the full spectrum of reactive channels.

Figure 1-66.

A chart to rationalize the photoreactive preferences of azabenzenes based upon thermodynamic criteria. The inability of benzene to show similar behavior can be linked to the energetic demand of the Dewar cycloreversion product and stronger tendency to funnel down to the ground state via internal conversion. Valence isomers play a key role in ring rearrangment processes occurring in the diazines and triazines.

Figure 1-67. 
One possible approach geometry for a $\mathrm{HCN} / \mathrm{CO} 22$ collision. Of significance is the magnitude of $\mathbf{v}$ and $\mathbf{g}_{\text {rel, }}$ which can be derived from $\mathrm{P}\left(\mathrm{E}_{\text {trans }}\right)$. A large fraction of $\mathrm{HCN}$ 's translational energy could be repartitioned (transferred) following a single collision with $\mathrm{CO}_{2}$. Other collision trajectories (and internal excitations) will favor different ways of partitioning the energy into the system's degrees of freedom.

Figure 1-68.

A simple estimate of the amount of rotational excitation possible, with analogy to an impulsive triatomic dissociation (see ref. 55). For the fastest $\mathrm{HCN}$ carrying $36 \mathrm{kcal} / \mathrm{mol}$, the rotational excitation into $\mathrm{CO}_{2}$ could reach up to $0.5 \mathrm{eV}$. Values up to $0.3 \mathrm{eV}$ have been measured experimentally for the pyrazine/ $\mathrm{CO}_{2}$ system (ref. 46 ). 
Rotating Source Machine

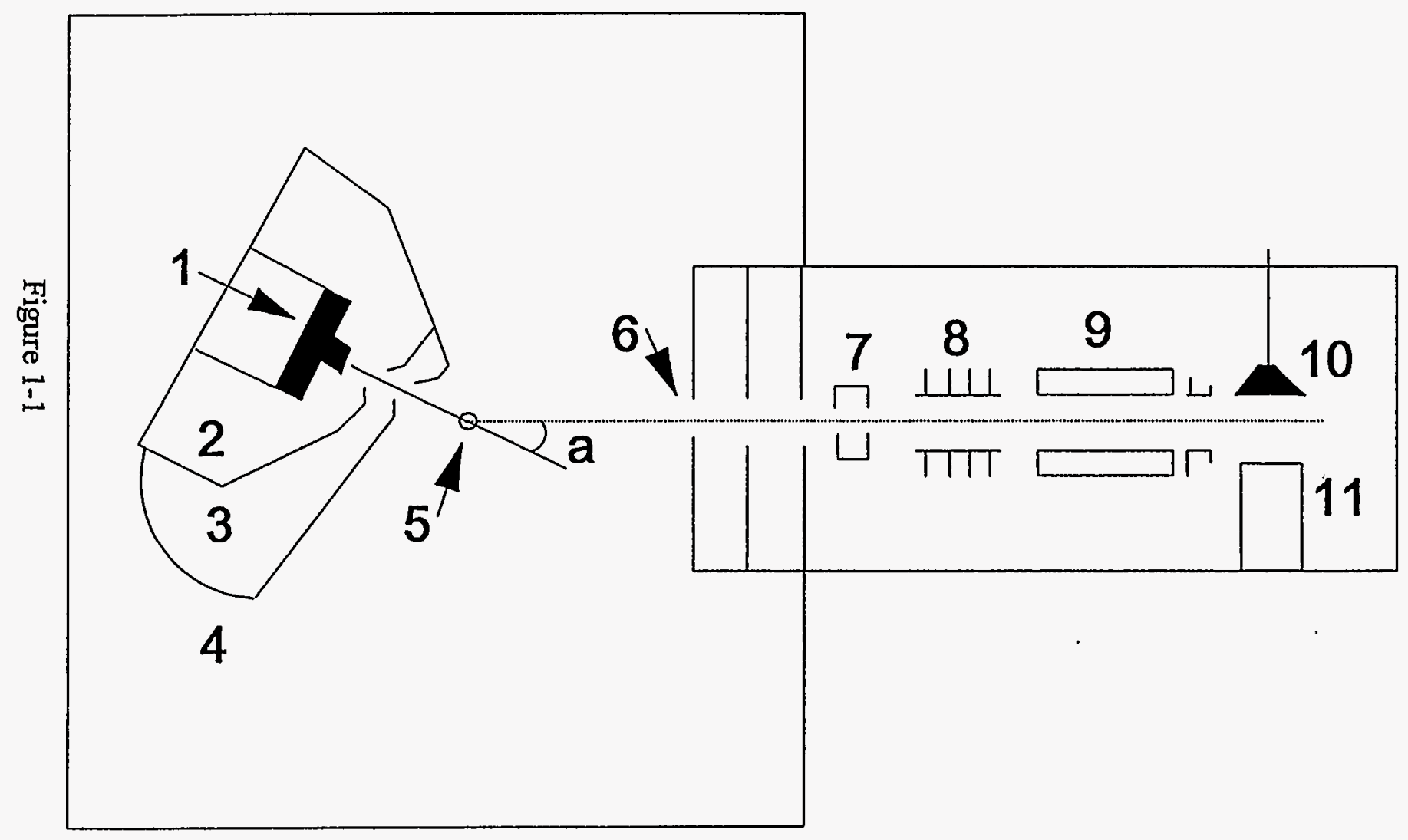




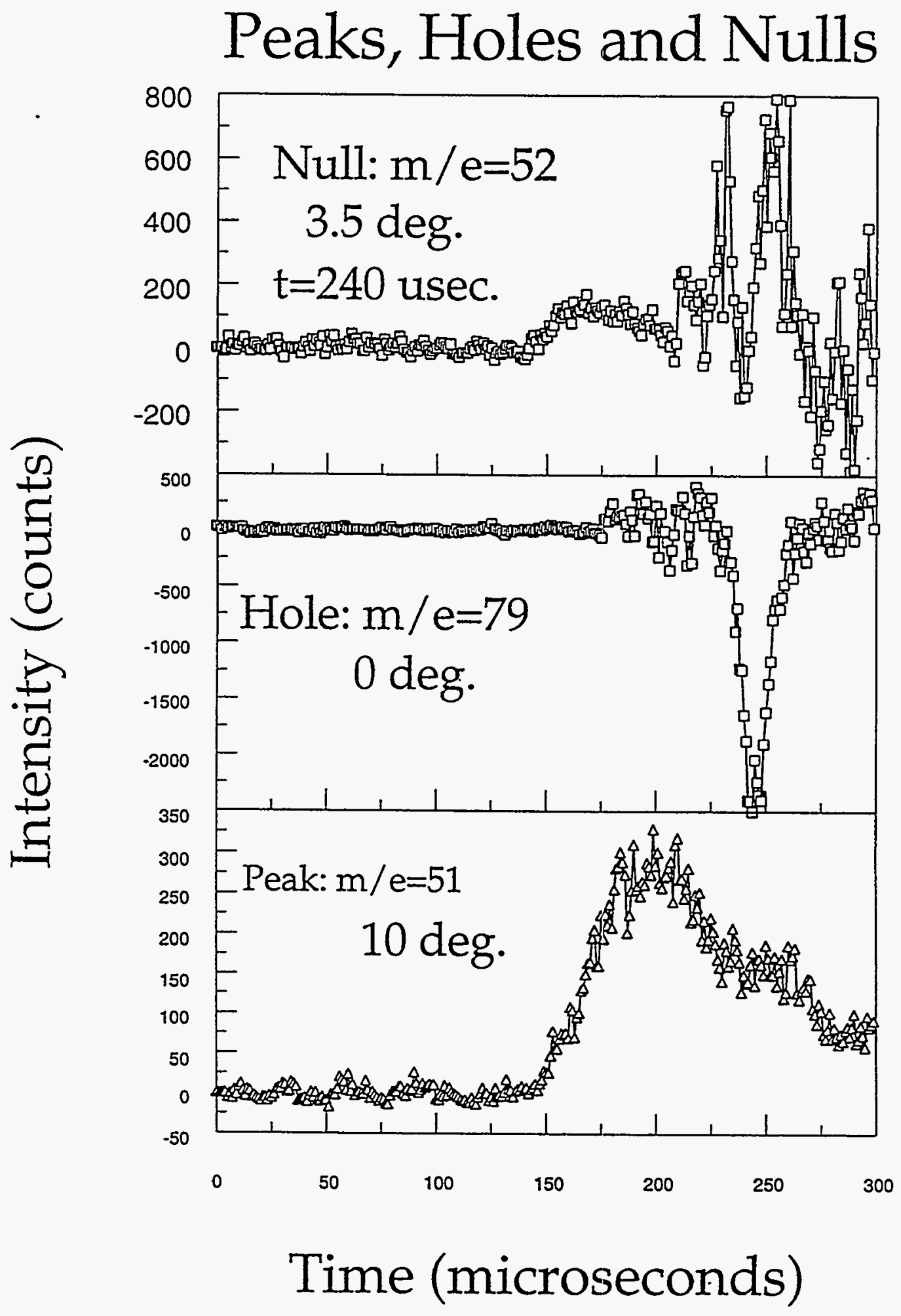

Figure 1-2 


\section{Holeburning Cross Section Measurement}
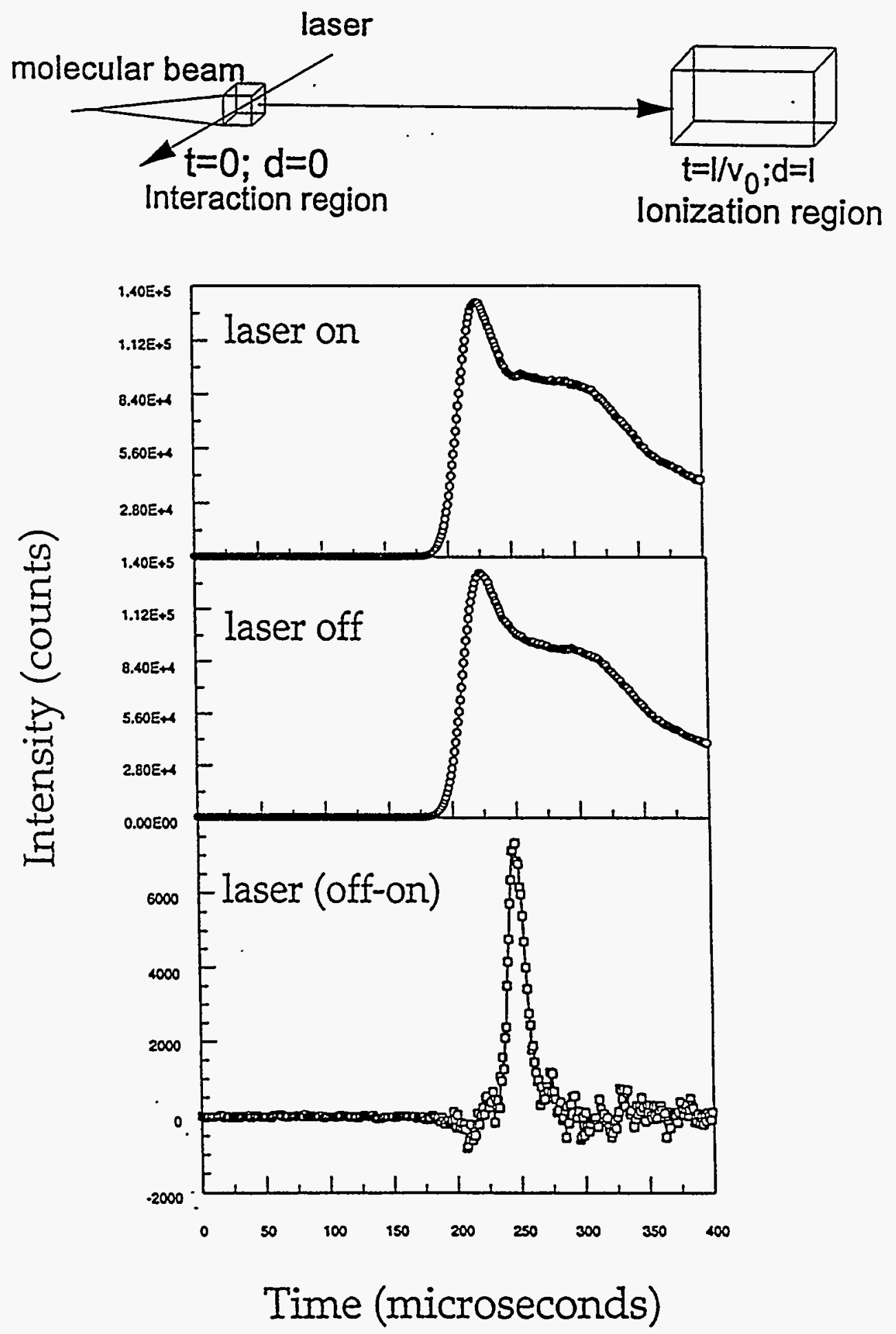

Figure 1-3 


\section{Ionization and Fragmentation. Pathways}

Mechanism:

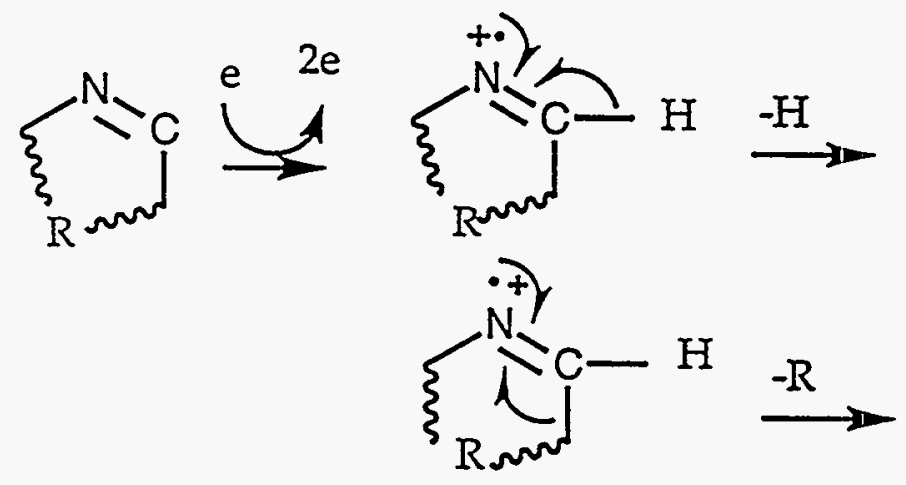

Typical fragmentation patterns:<smiles>[R]1CC#[N+]CC1</smiles>

$$
\begin{aligned}
& \mathrm{m} / \mathrm{e}=80 \stackrel{0.30}{\underset{0.25}{\longrightarrow}} 80+ \\
& 0.0652+ \\
& \underset{0.02}{0.01} 40 t \\
& \underset{0.30}{0.02} 27+ \\
& \mathrm{m} / \mathrm{e}=53 \frac{0.08}{0.29} 53+ \\
& \overrightarrow{0.31} 52+ \\
& \underset{0.03}{0.31} 51+/ 50+ \\
& \longrightarrow 27+ \\
& \frac{0.29}{0.01} 26+ \\
& \rightarrow 14+/ 13+/ 28+\text { etc. }
\end{aligned}
$$

$$
\mathrm{m} / \mathrm{e}=40 \stackrel{0.40}{\stackrel{0.38}{\longrightarrow}} 40+
$$

Figure 1-4 
Pyrazine VUV Absorption Spectra (ref. 10)

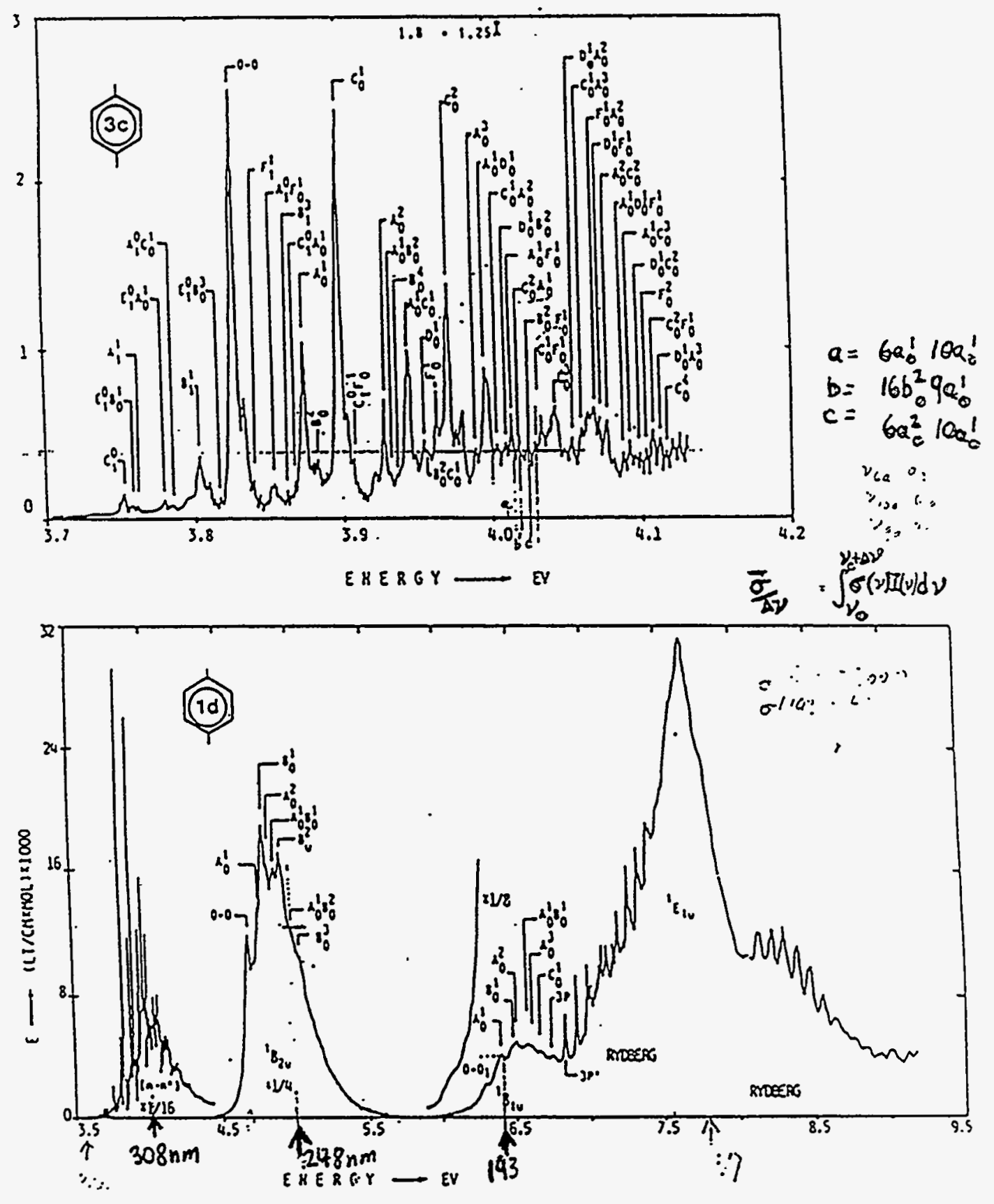

Figure 1-5 
Spectral Profiles of The Four Principal Excimer Laser. Wavelengths

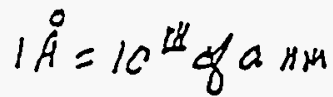

$=0.1 \mathrm{~nm}$

$\simeq 1^{105} \mathrm{NAH}$

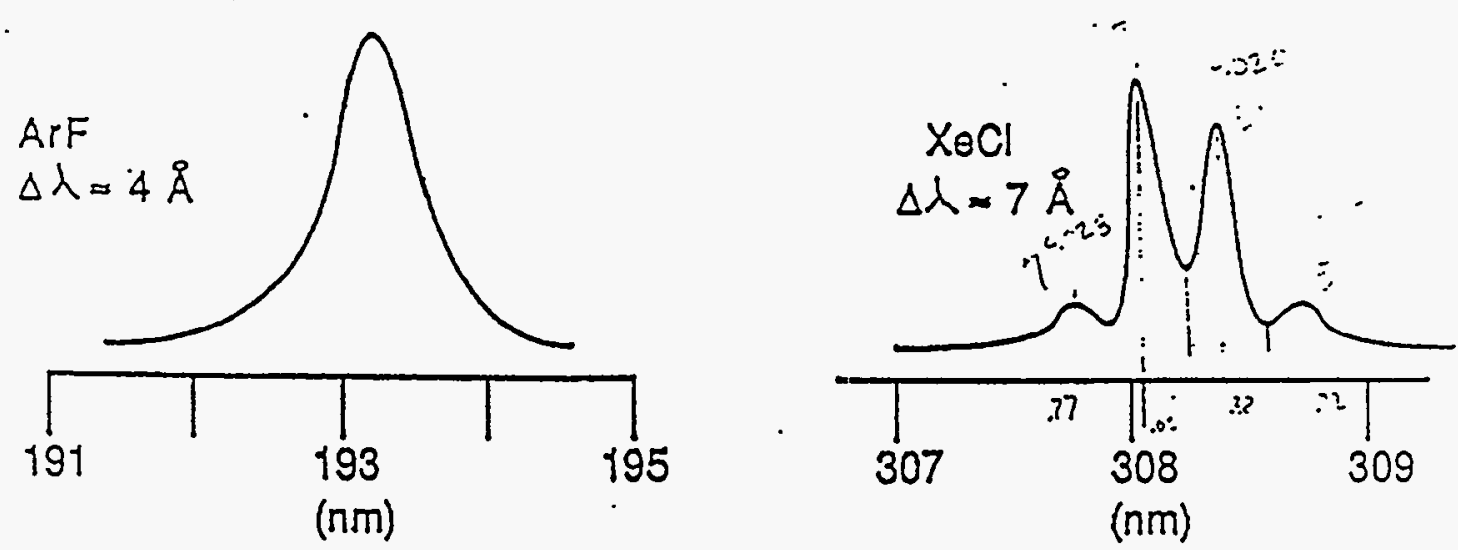

$4.025 \mathrm{e}$

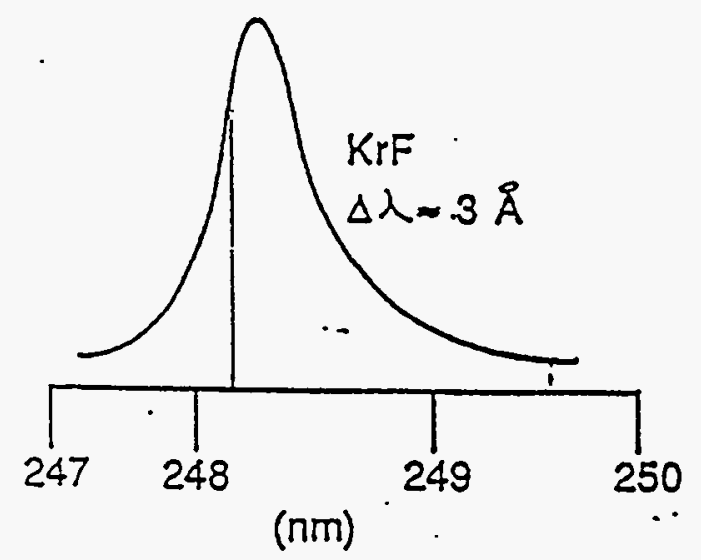

$\vdots$

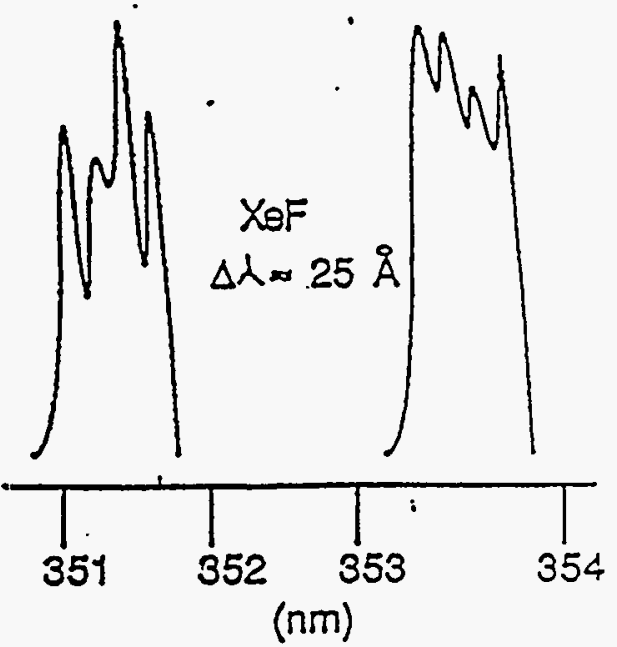

Figure 1-6 


\section{Dissociation Lifetime vs. Barrier Height}

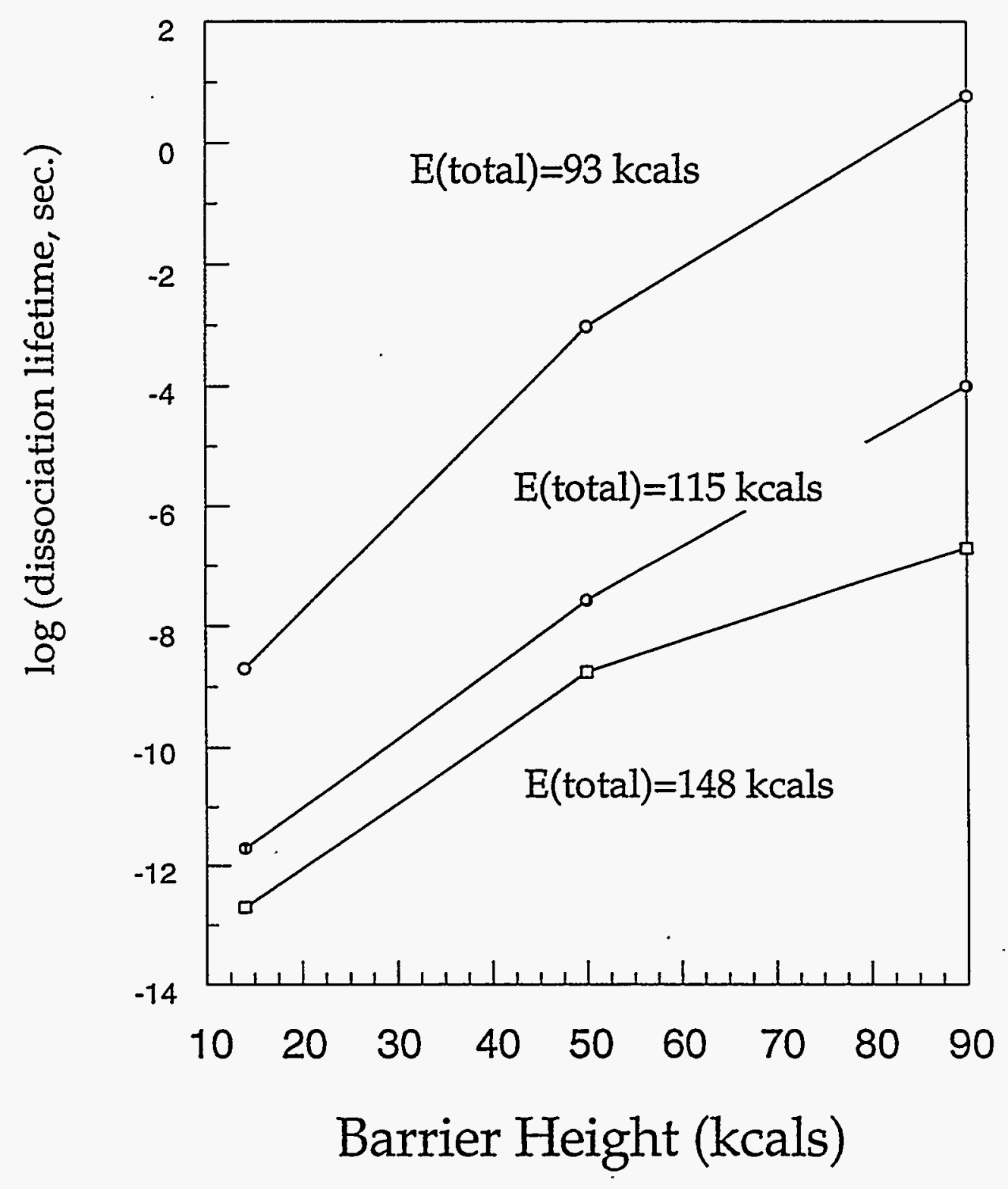

Figure 1-7 


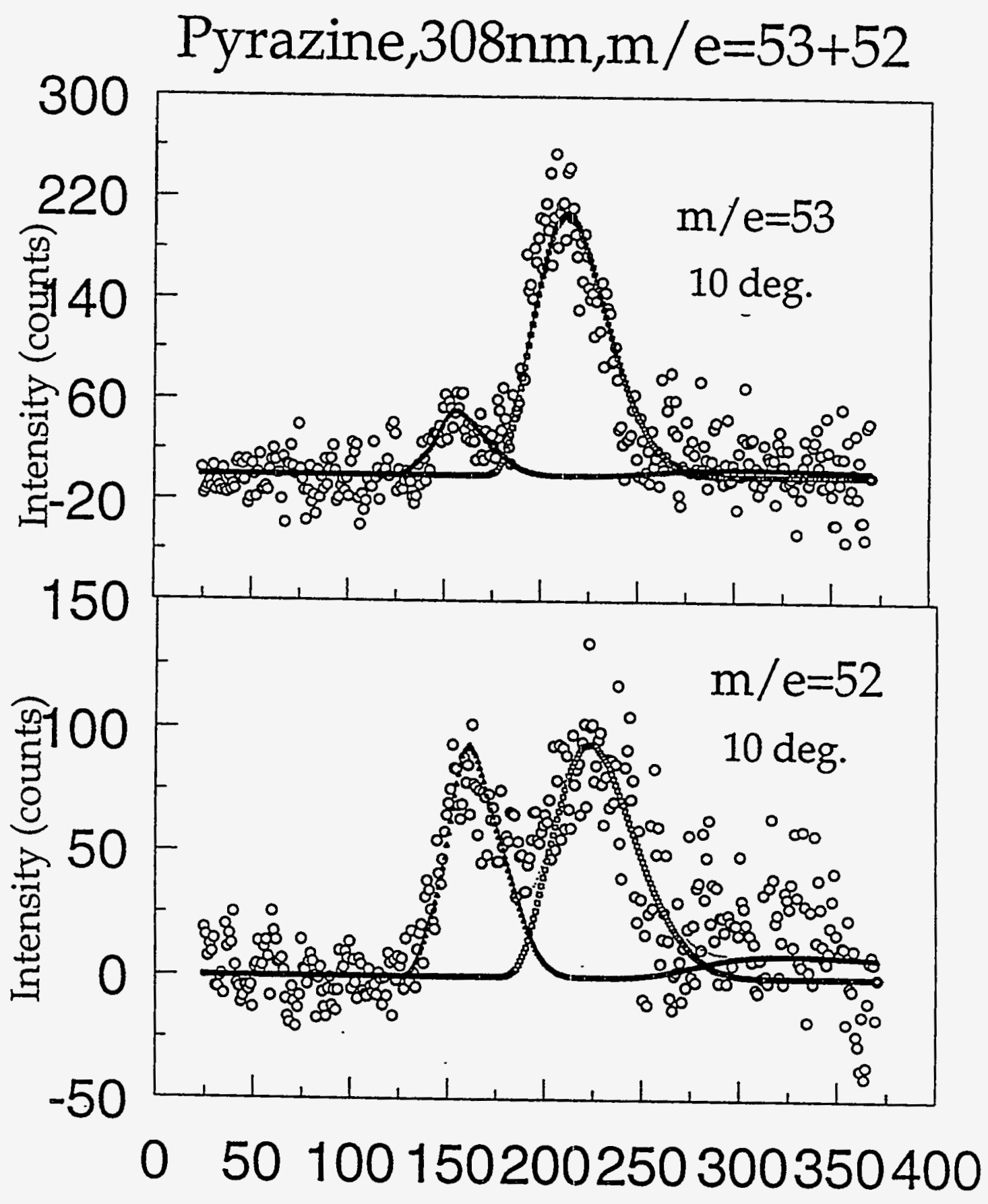

Time (microseconds)

Figure 1-8 


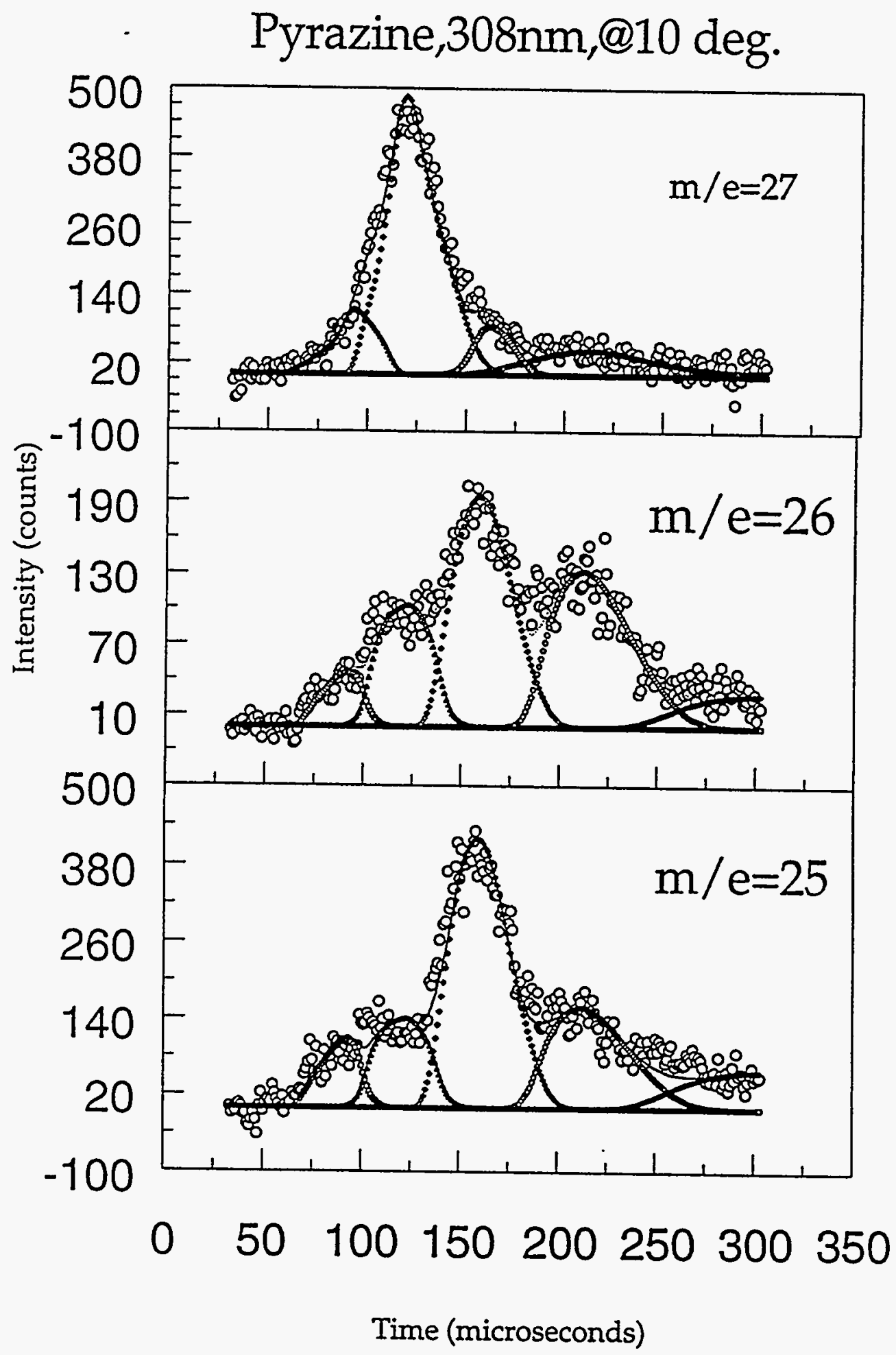

Figure 1-9 
Pyrazine, 308nm, $\mathrm{m} / \mathrm{e}=26$

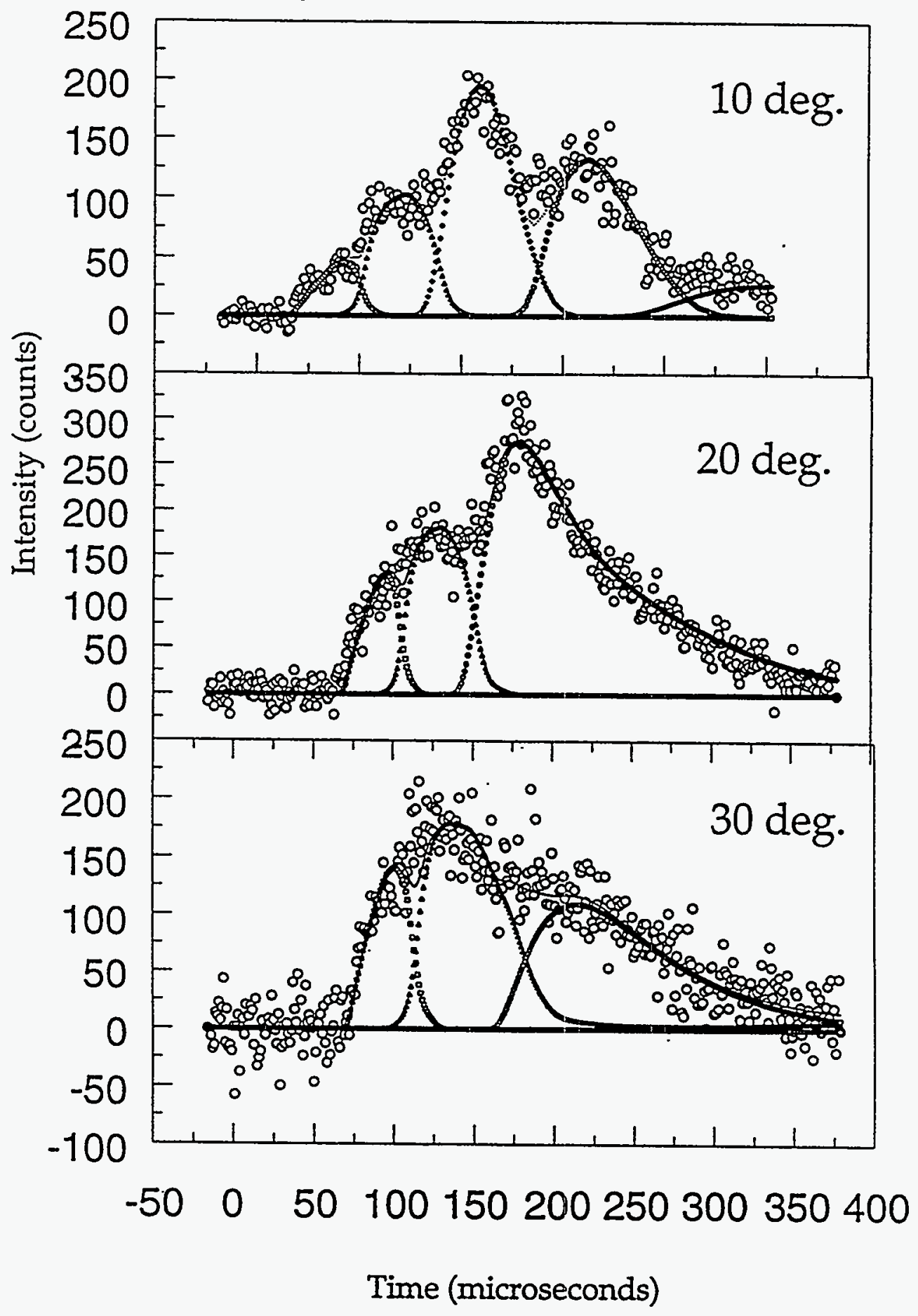

Figure 1-10 

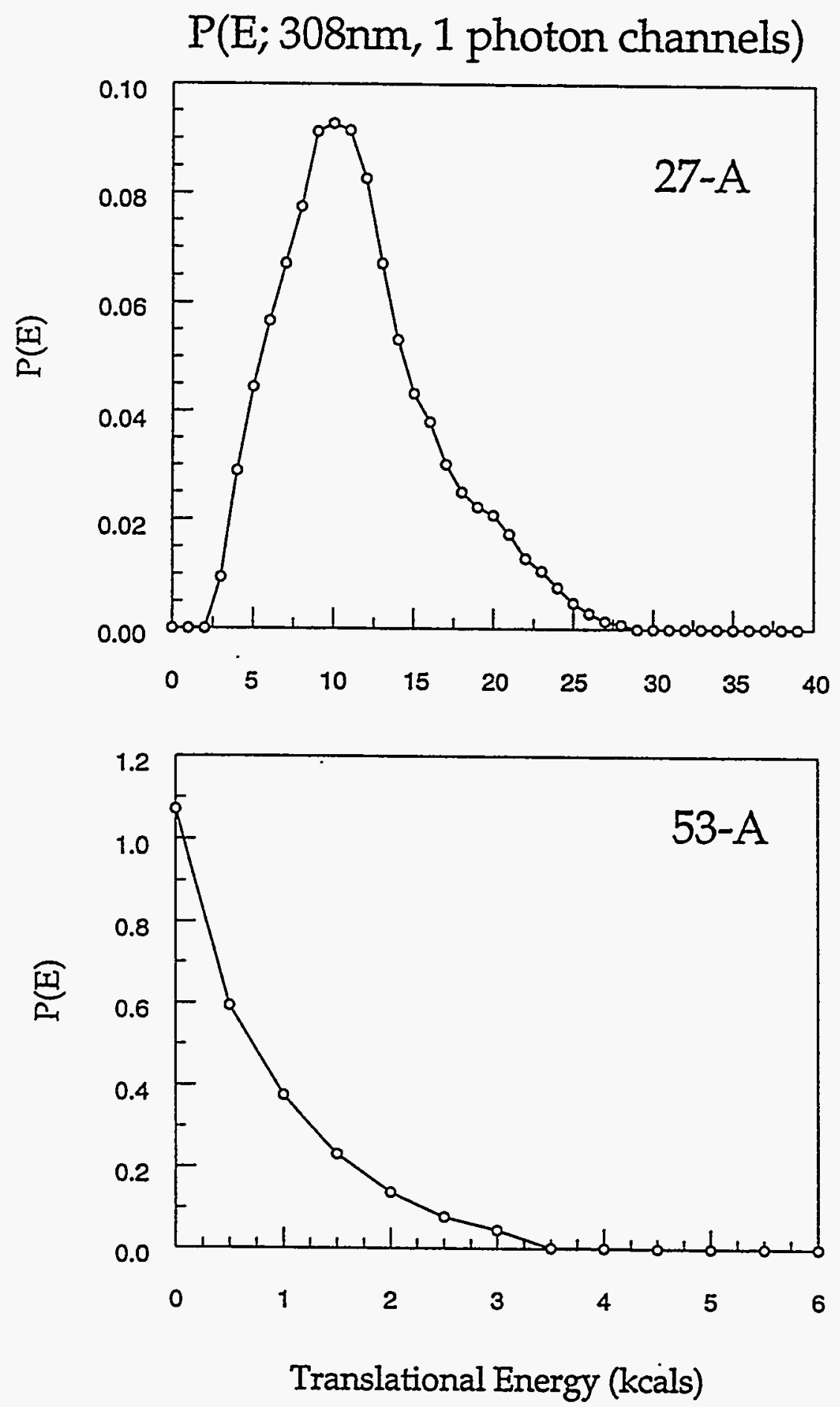

Figure 1-11 

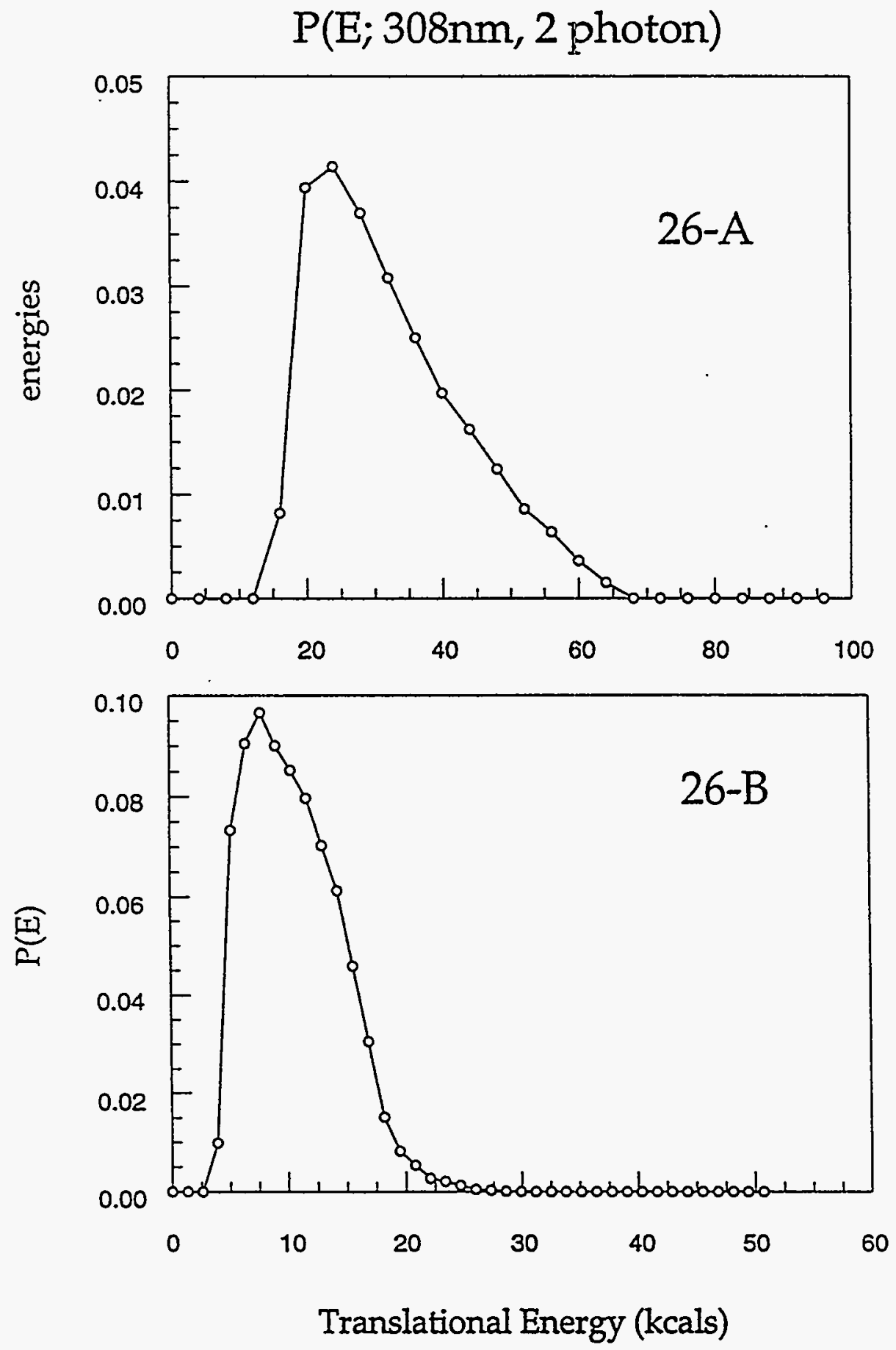

Figure 1-12 
Power Dependence, 308nm Channels

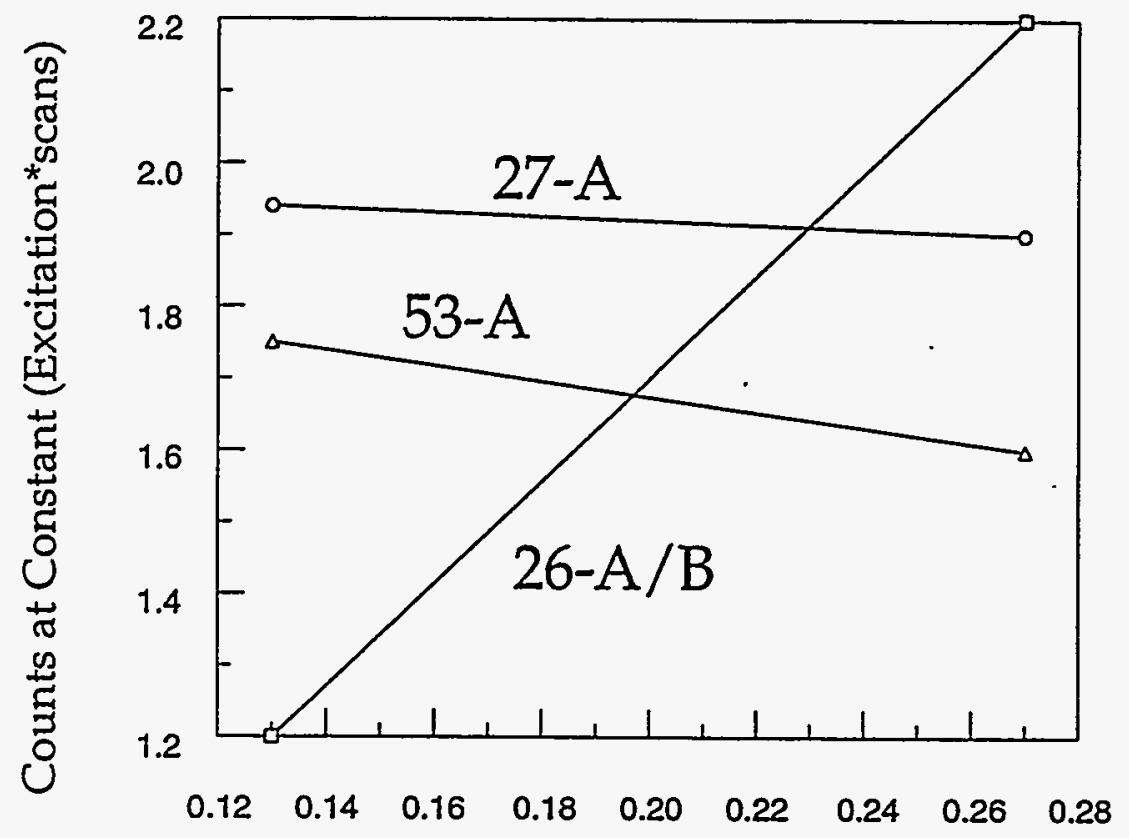

First Excitation Probability

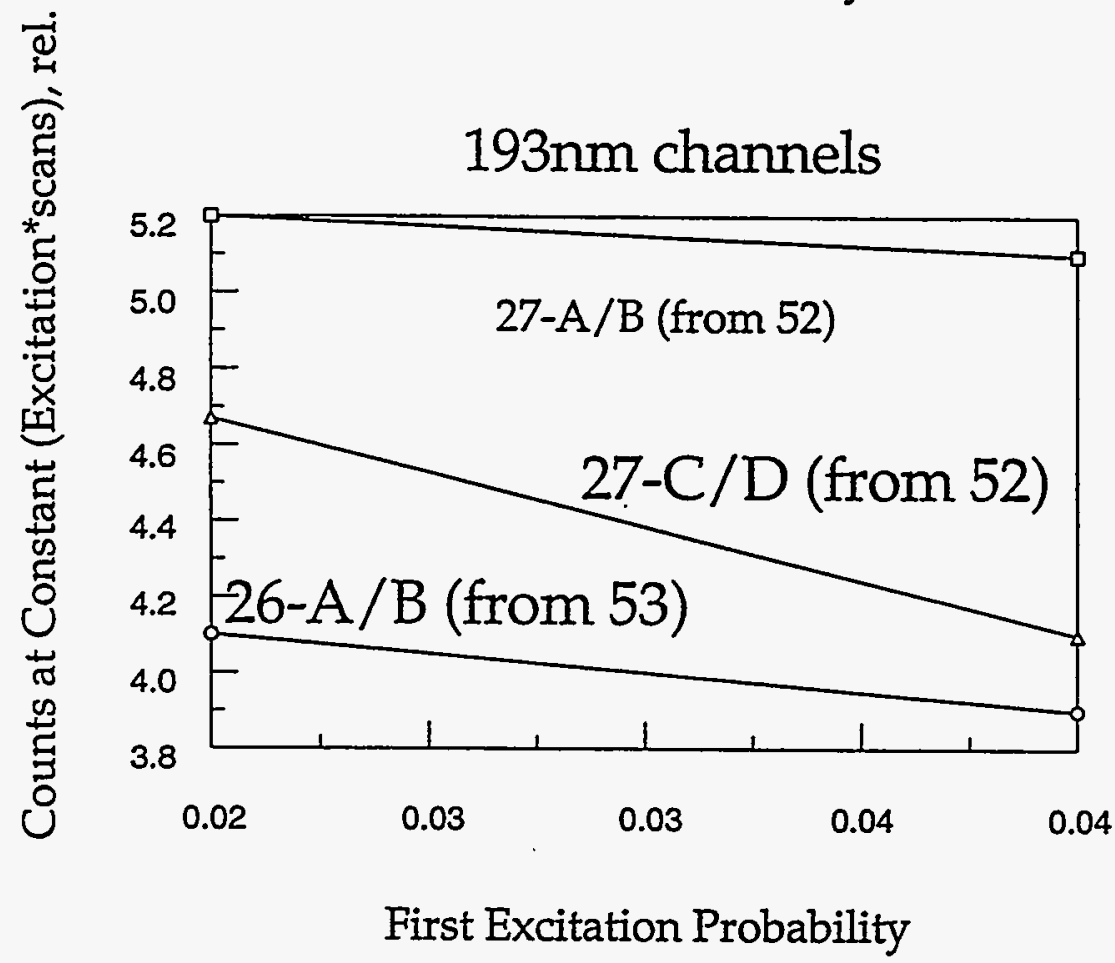

Figure 1-13 


\section{Power Dependence, Pyrazine, 248nm}

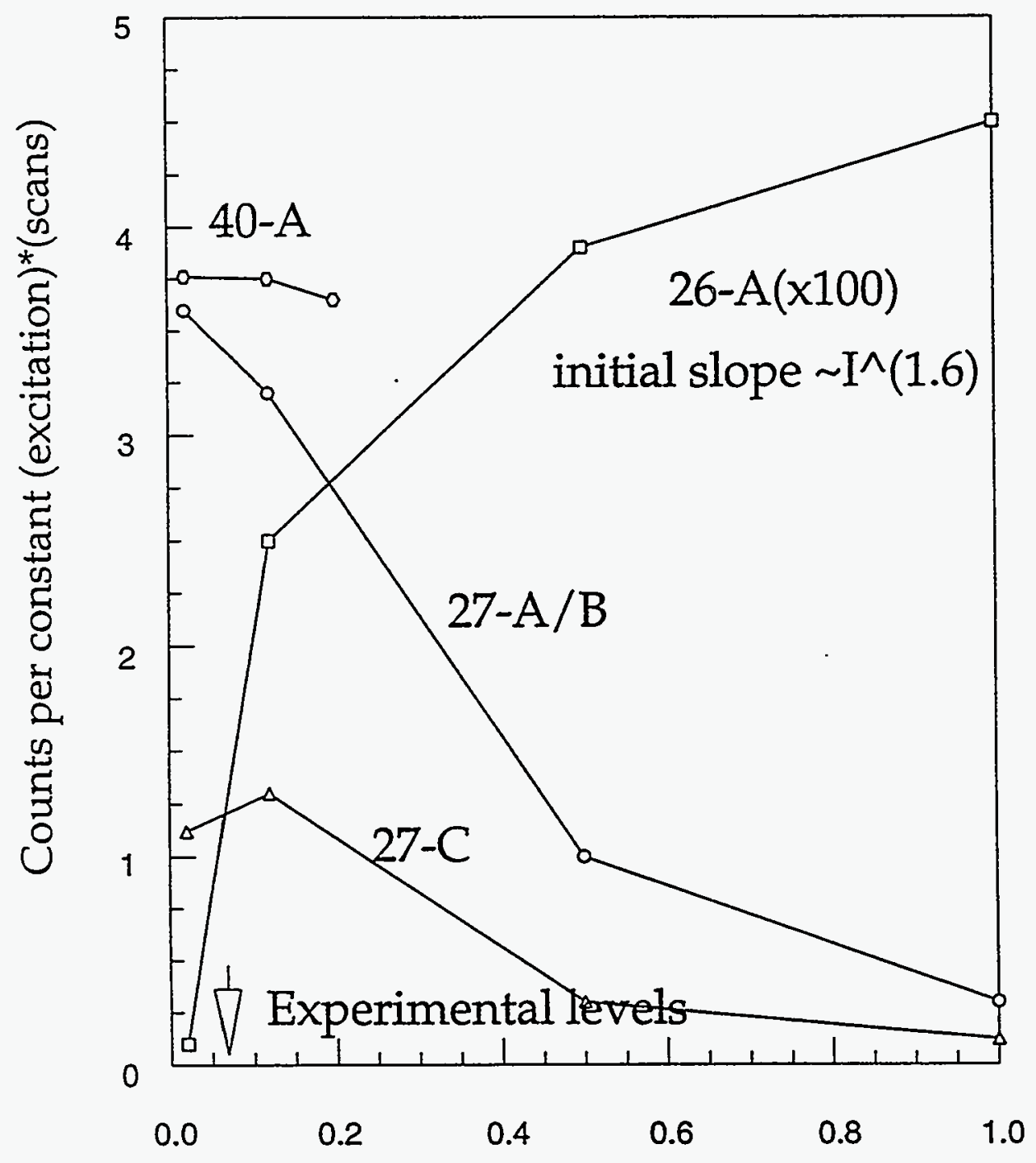

First excitation probability

Figure 1-14 


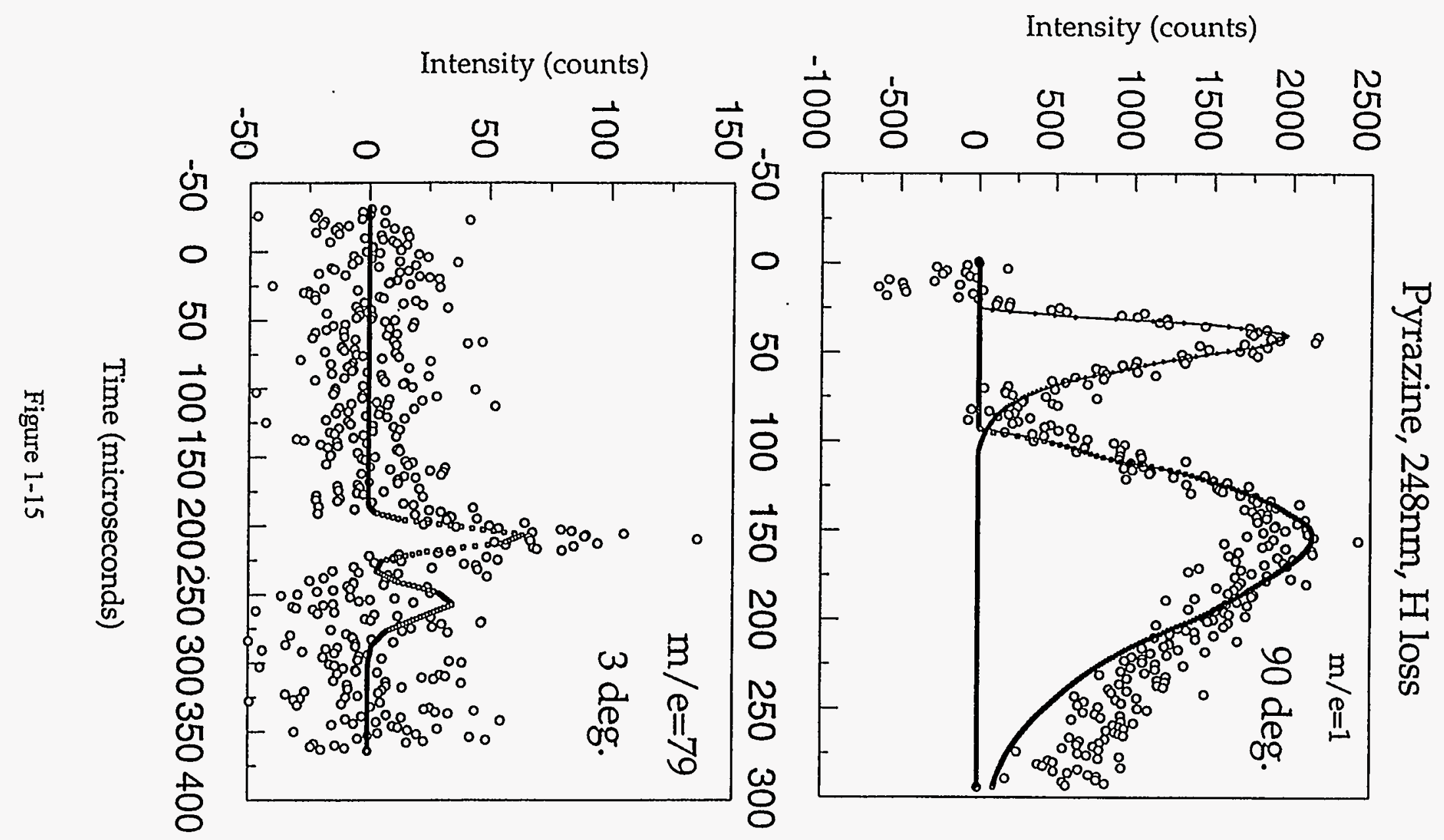


Pyrazine, 248nm, H2 loss

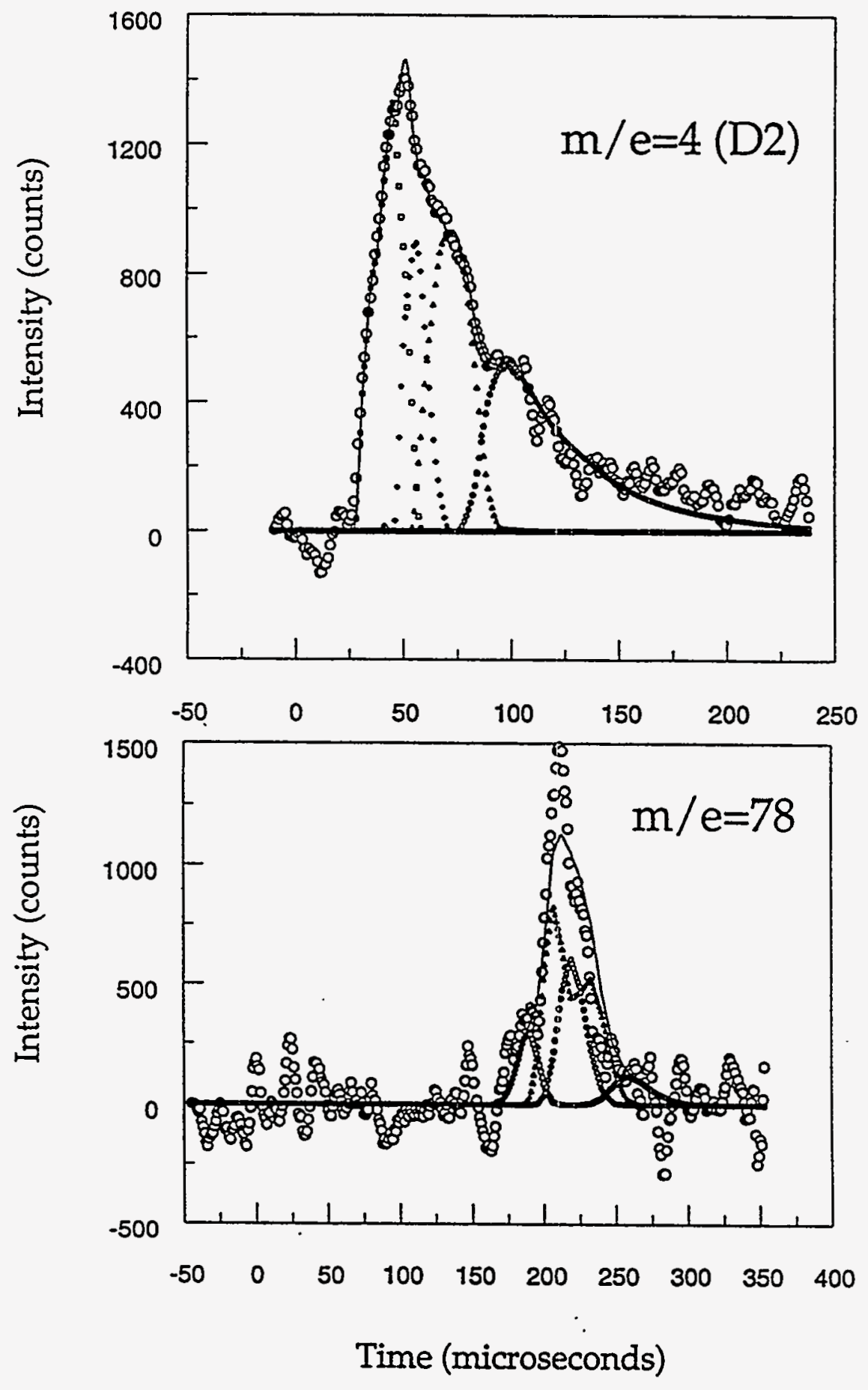

Figure 1-16 


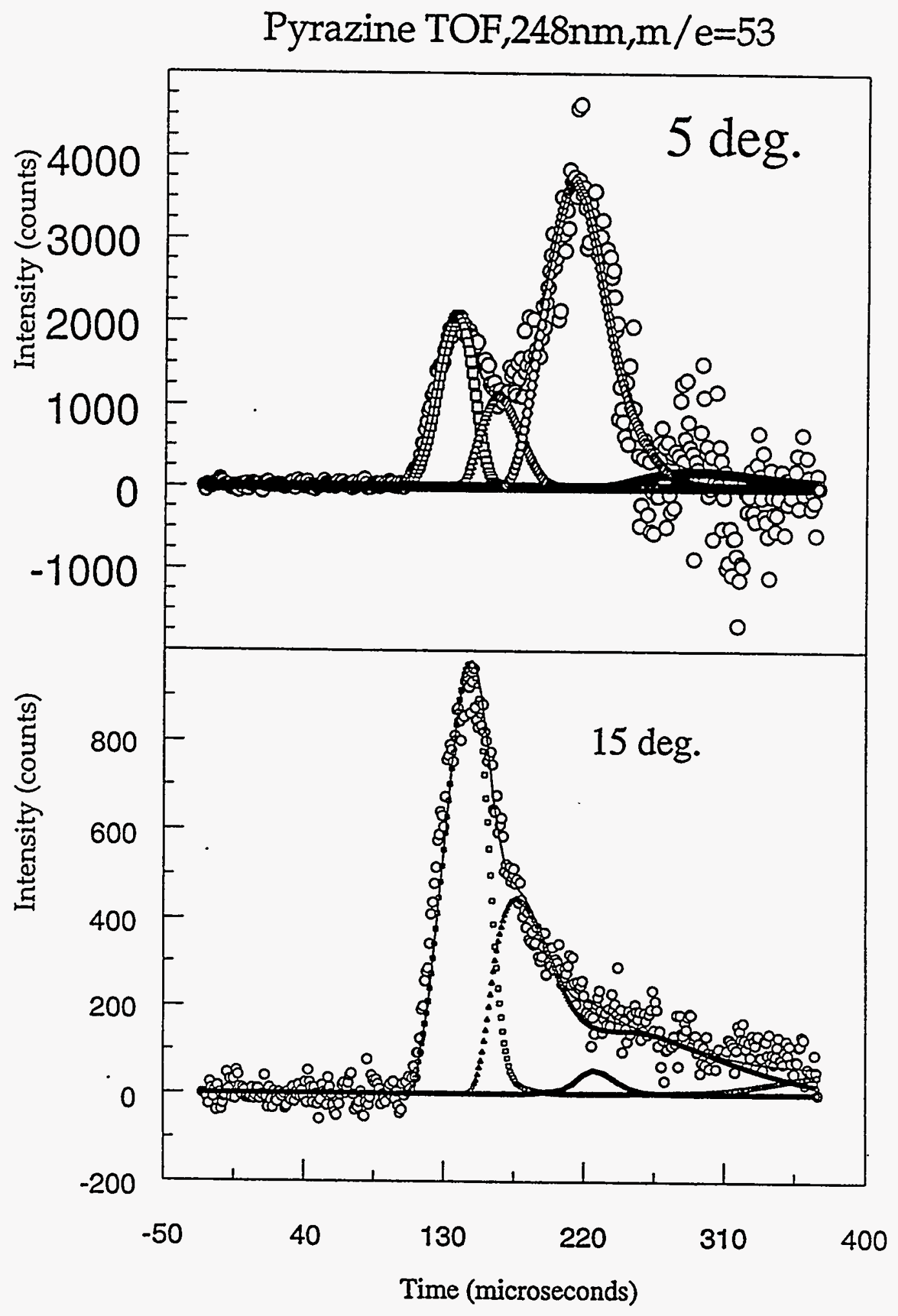

Figure 1-17 
Pyrazine, $248 \mathrm{~nm}, \mathrm{~m} / \mathrm{e}=52$

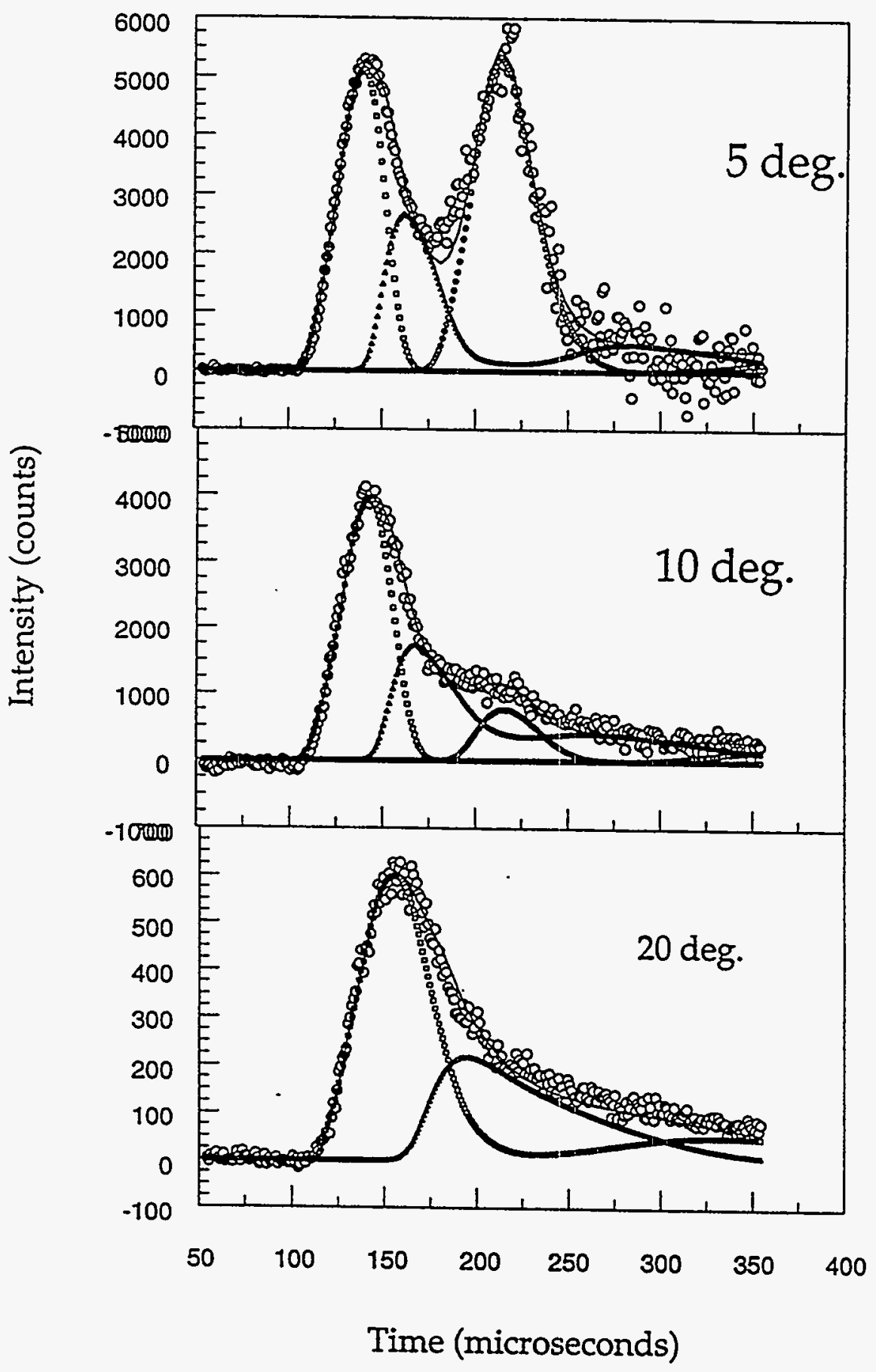

Figure 1-18 


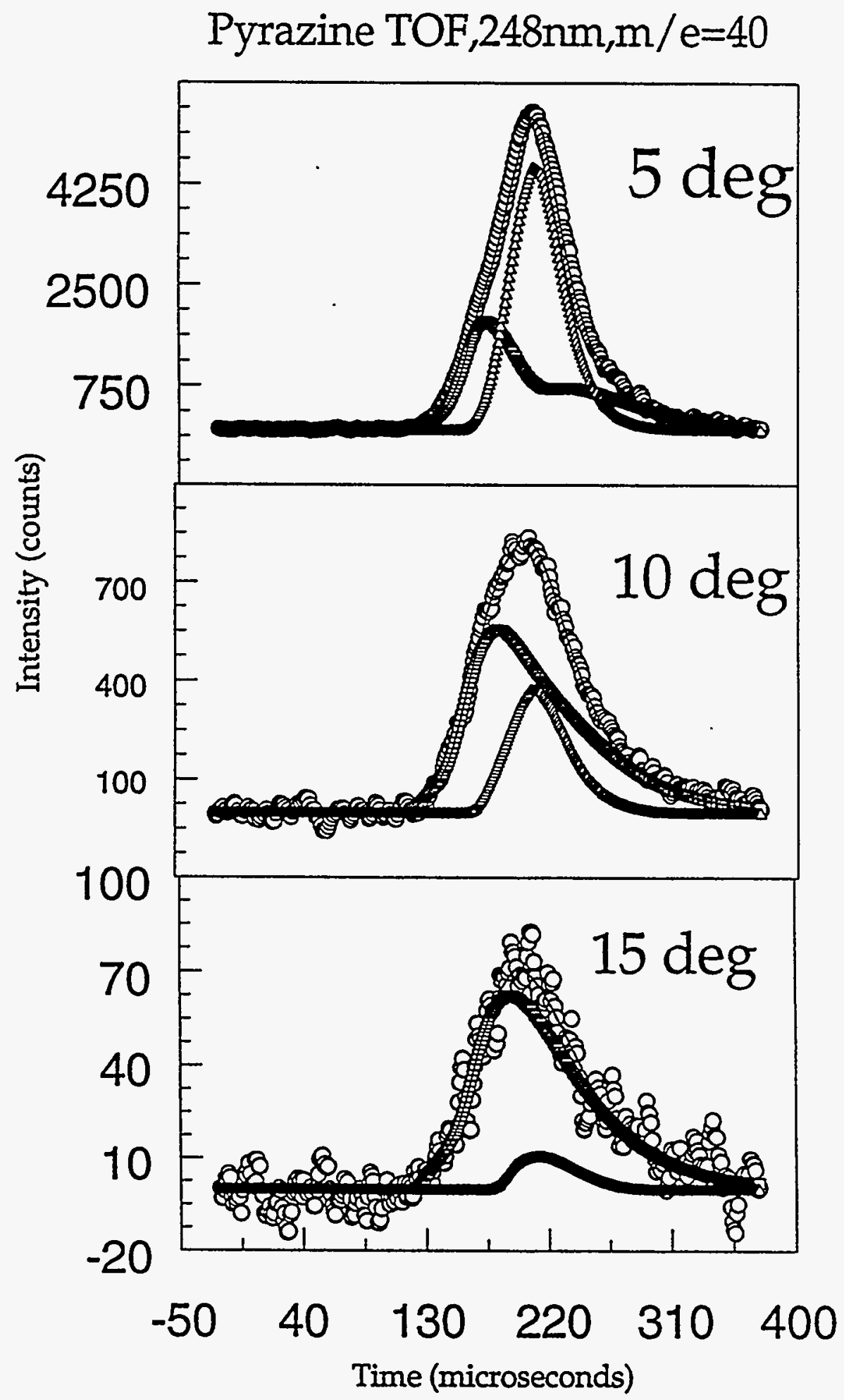

Figure 1-19 
Pyrazine TOF,248nm,m/e=27

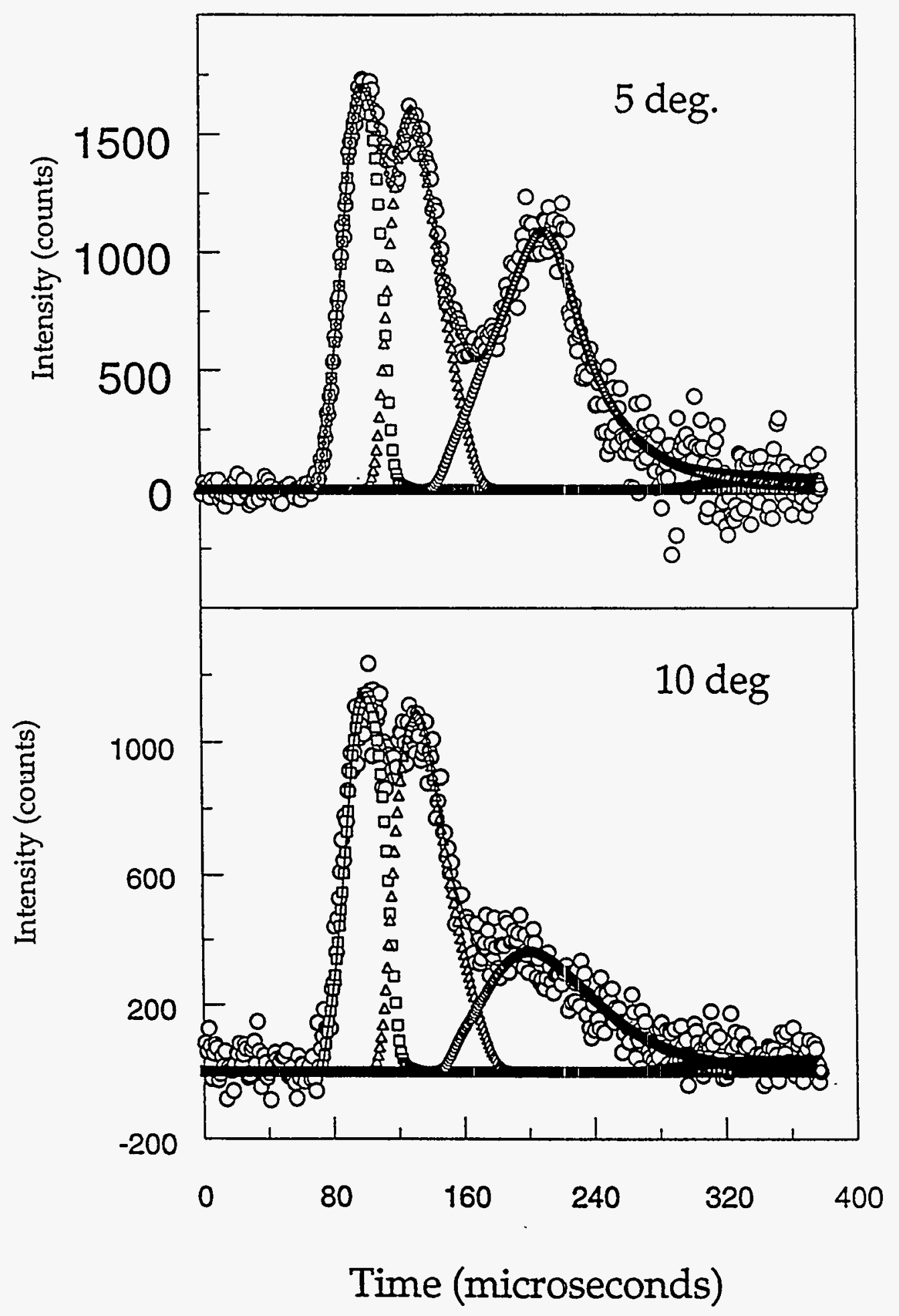

Figure 1-20 
Pyrazine TOF,248nm,m/e $=27$

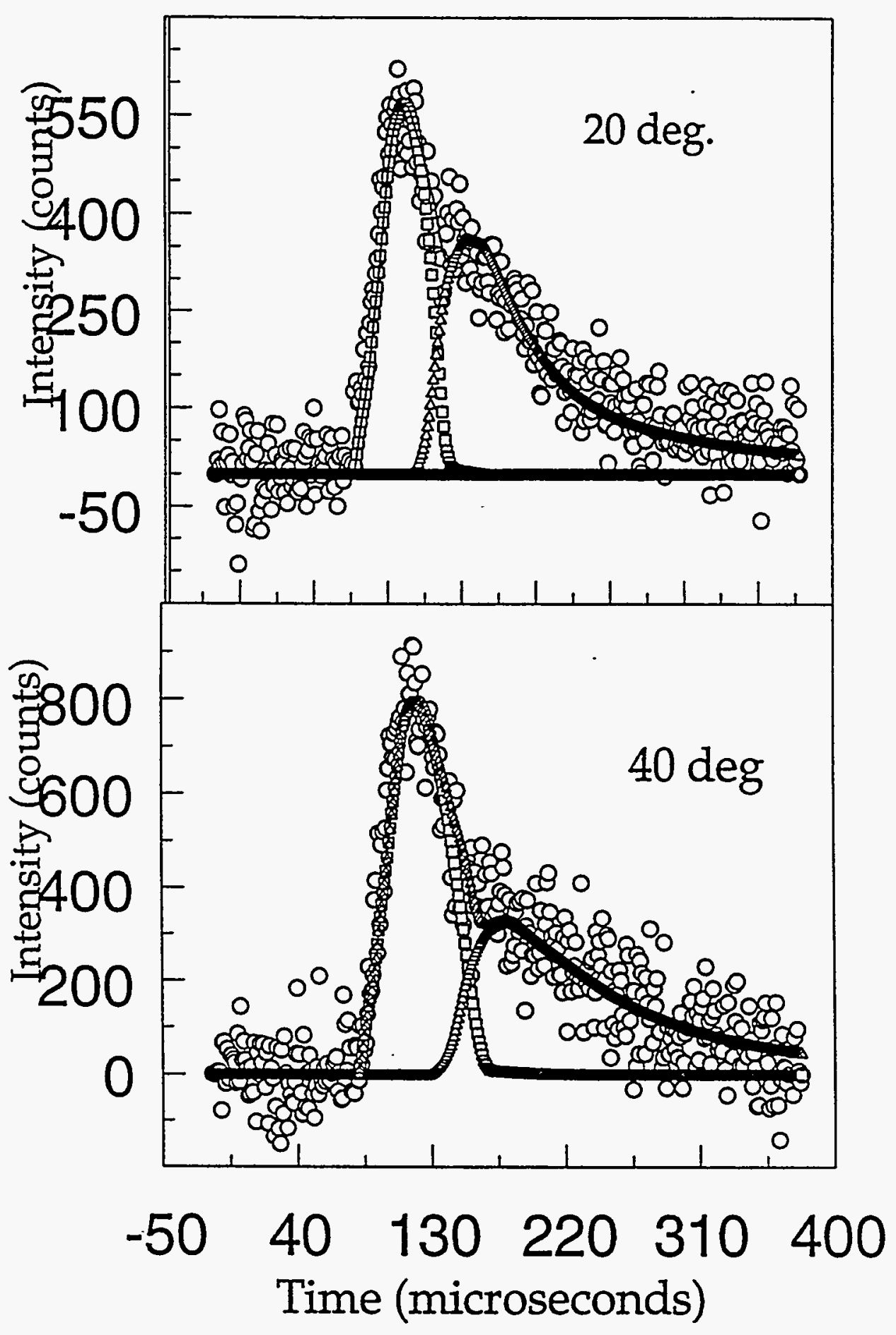

Figure 1-21 


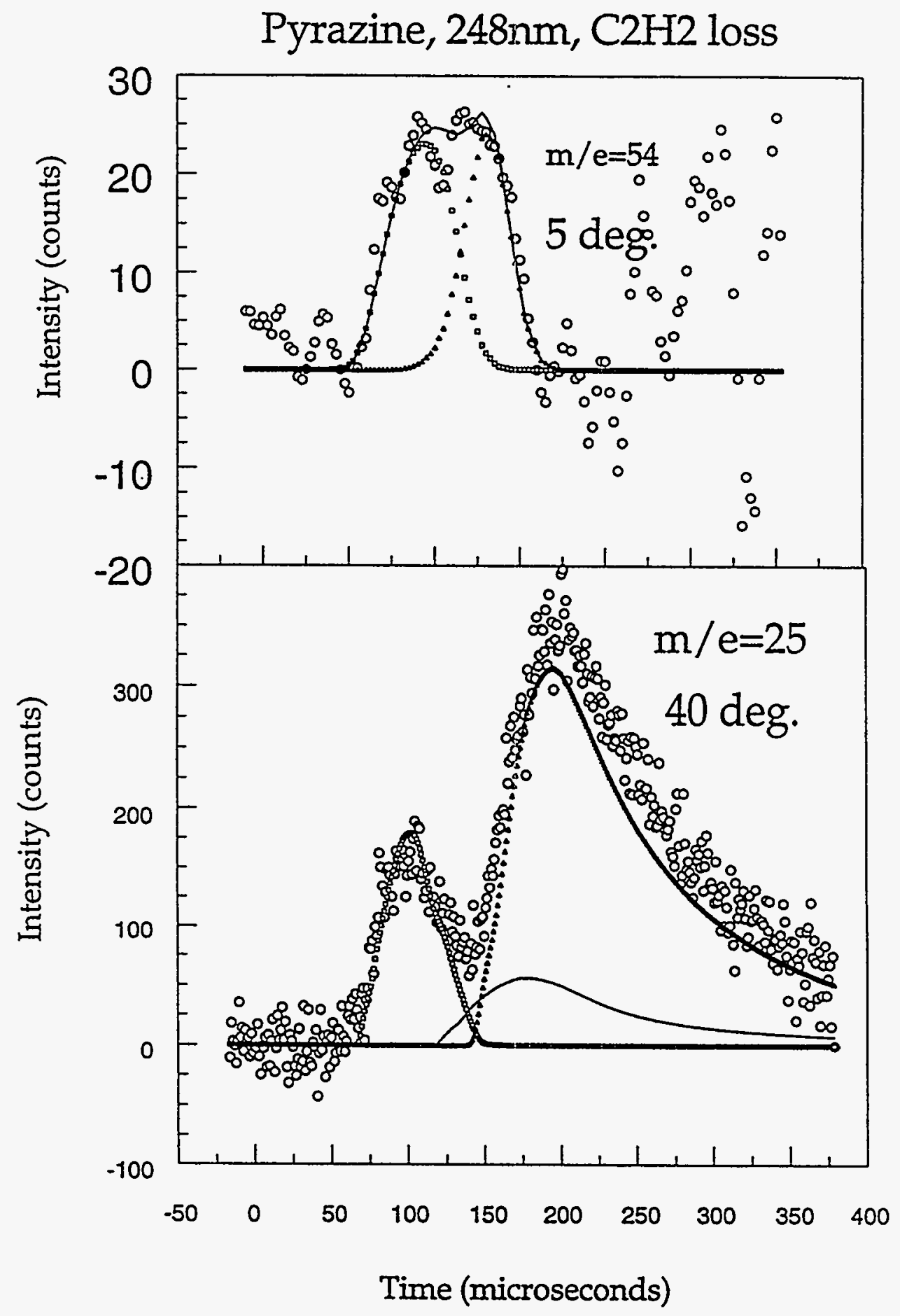

Figure 1-22 
Pyrazine, 248nm, N2 loss

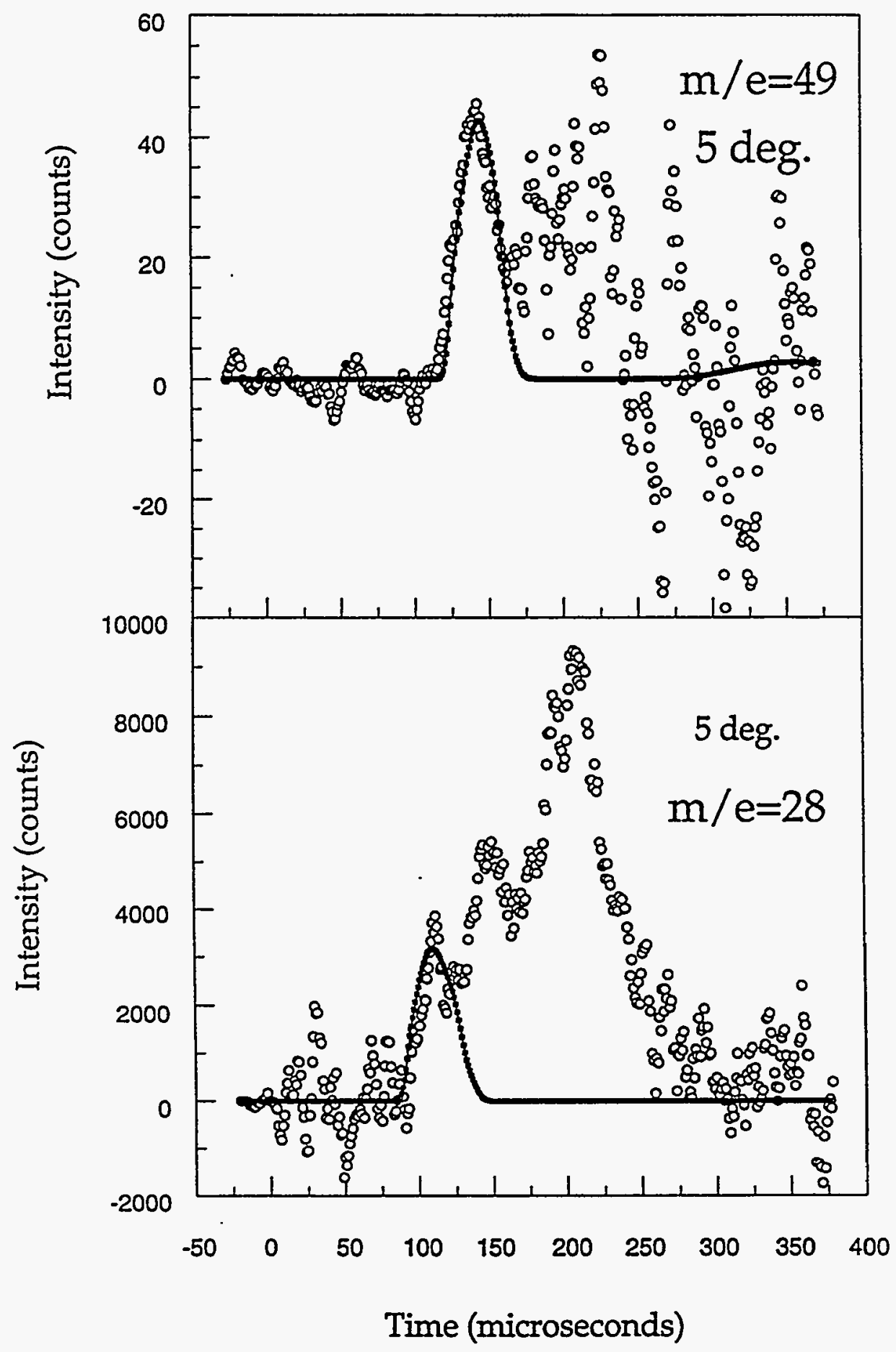

Figure 1-23 


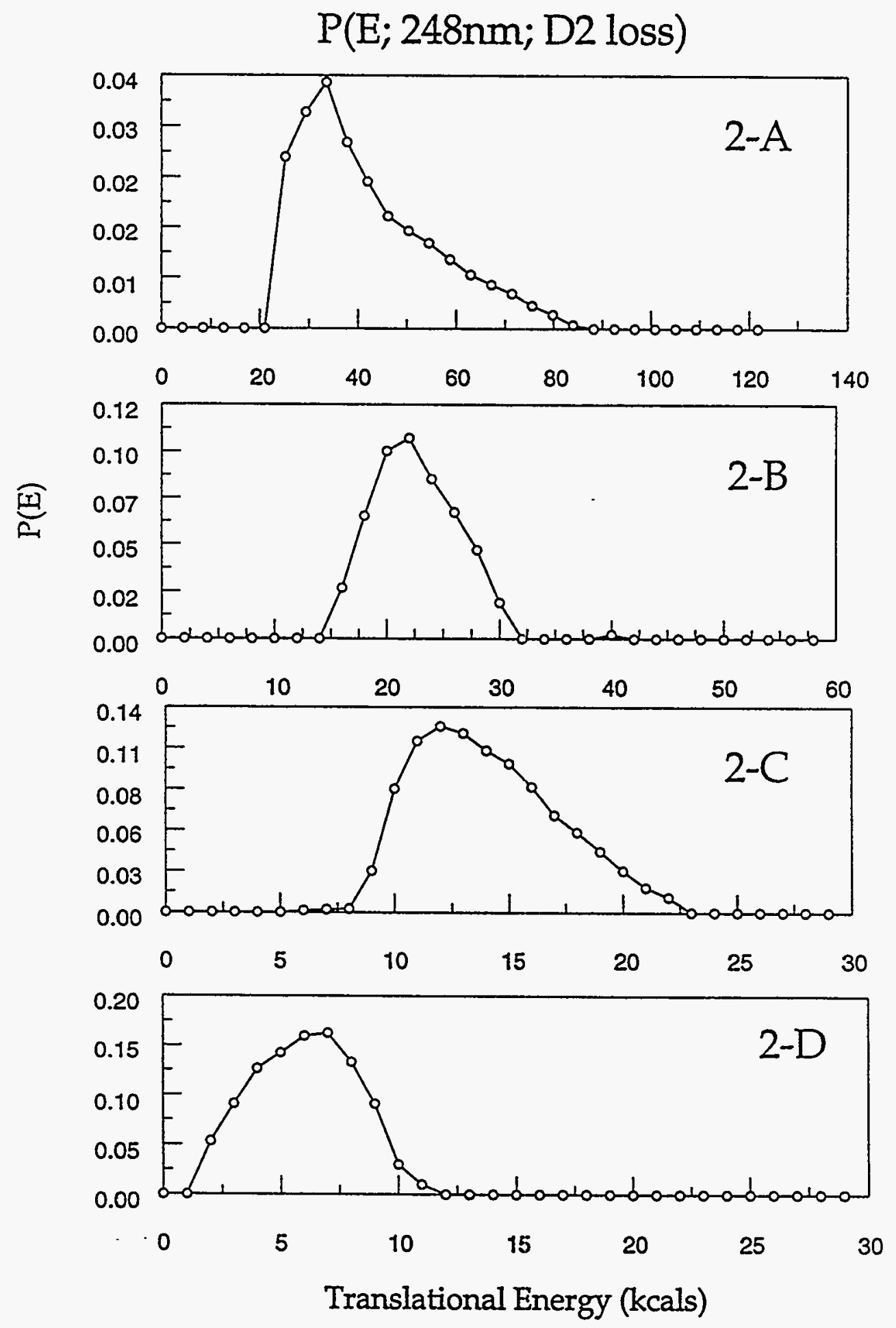

Figure 1-24 

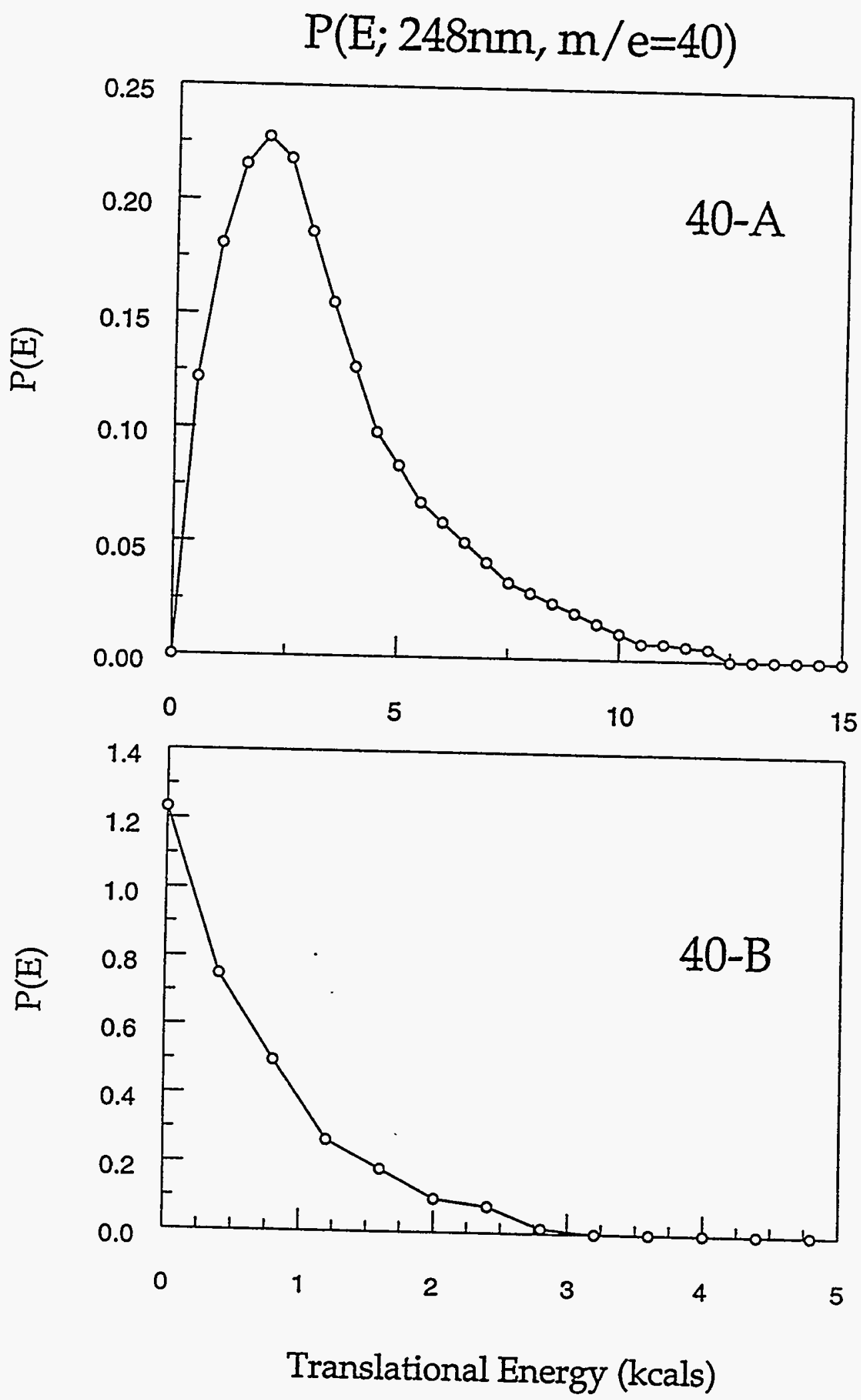

Figure 1-25 
$\mathrm{P}(\mathrm{E}$; 2-photon 248nm)
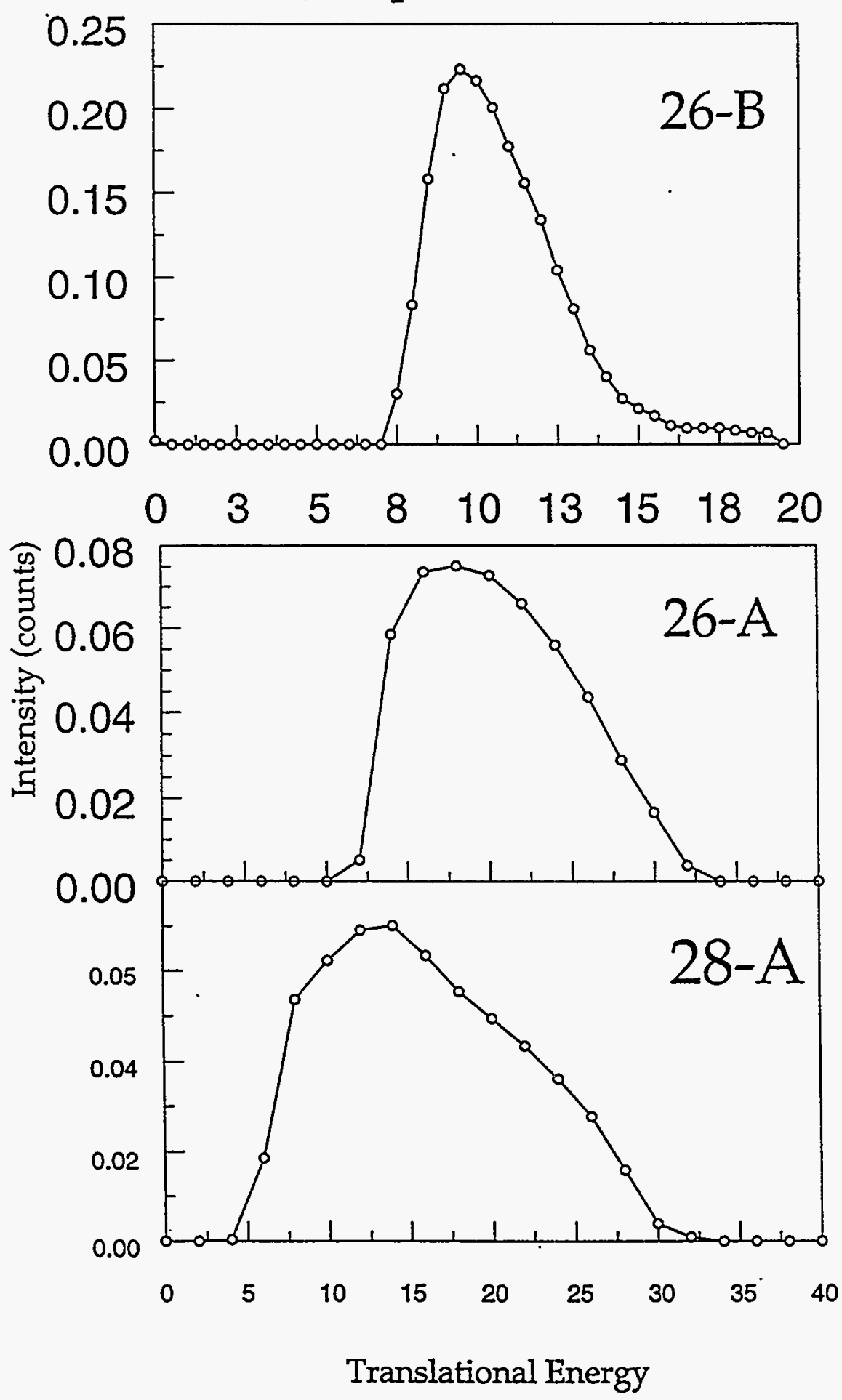

Figure 1-26 

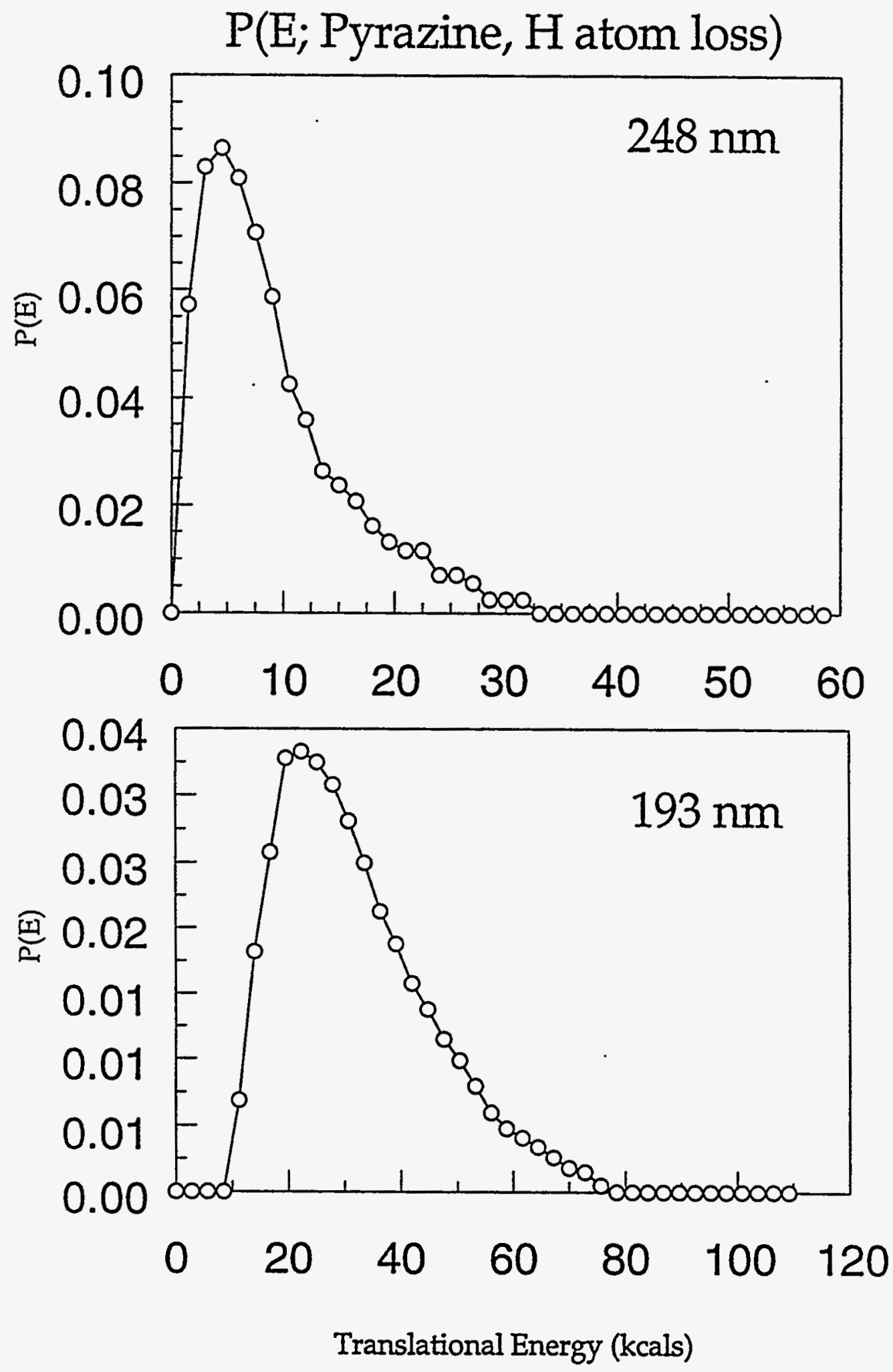

Figure 1-27 

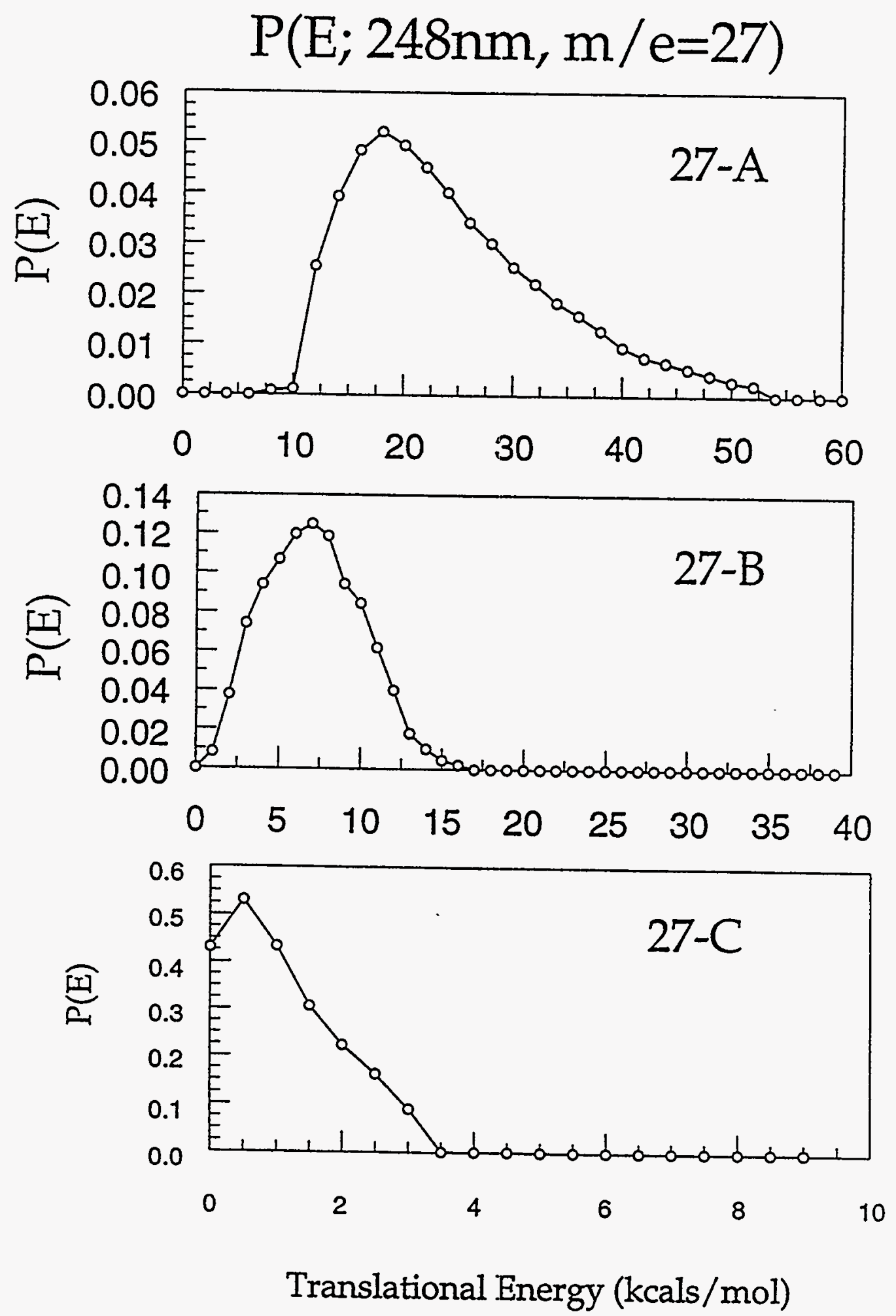

Figure 1-28 

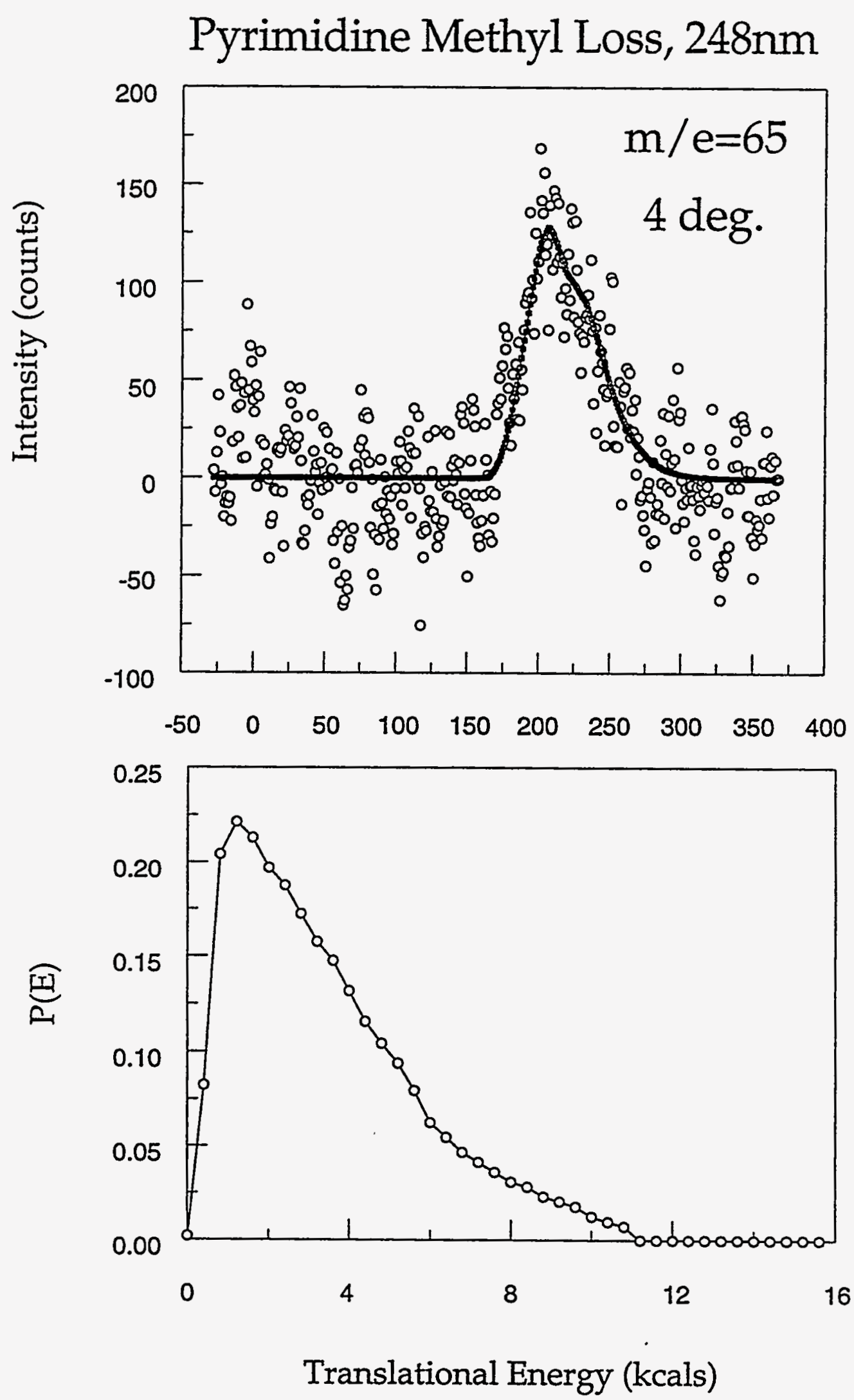

Figure 1-29 


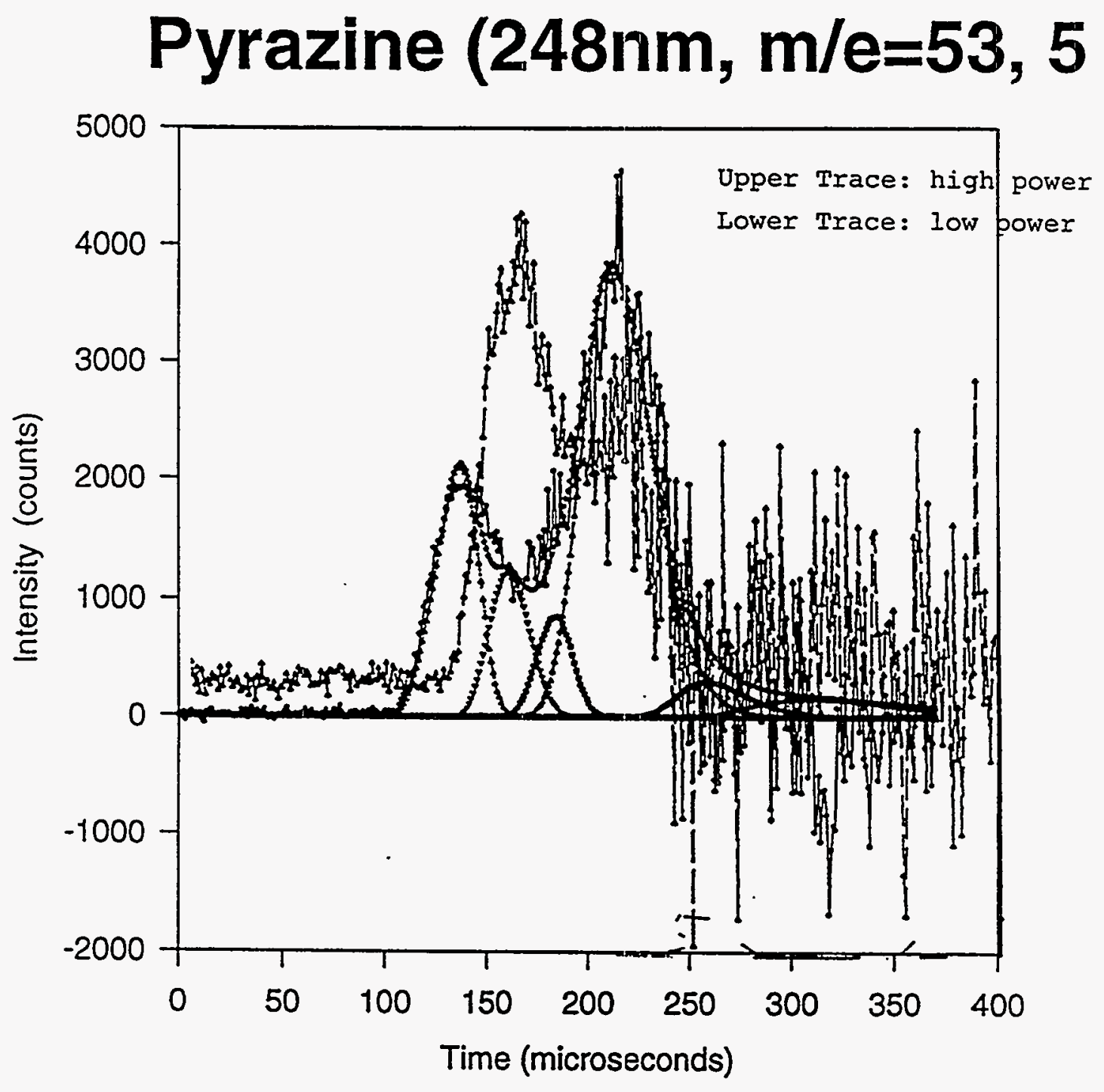

Figure 1-30 


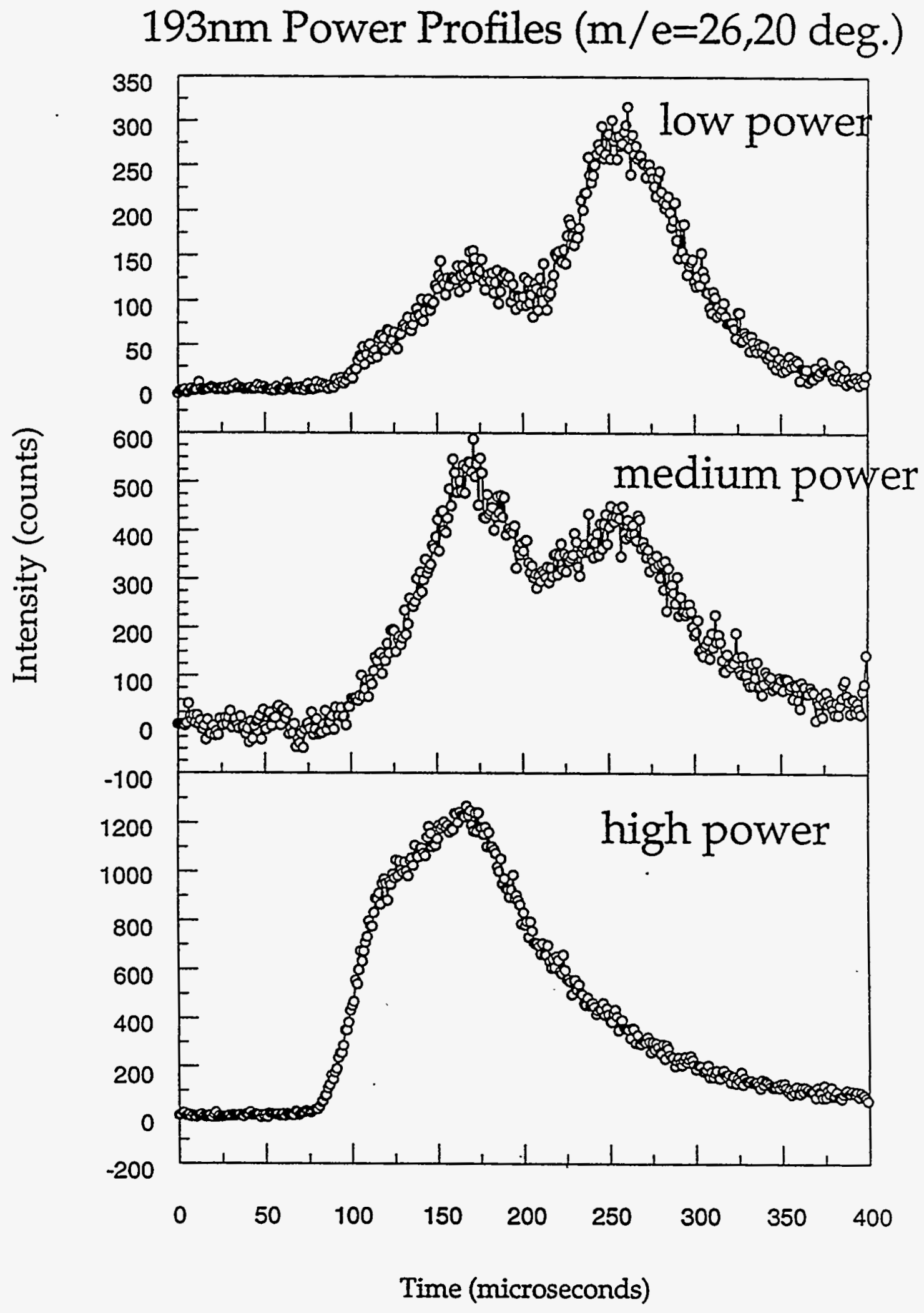

Figure 1-31 
Pyrazine, $193 \mathrm{~nm} \mathrm{H}$ loss
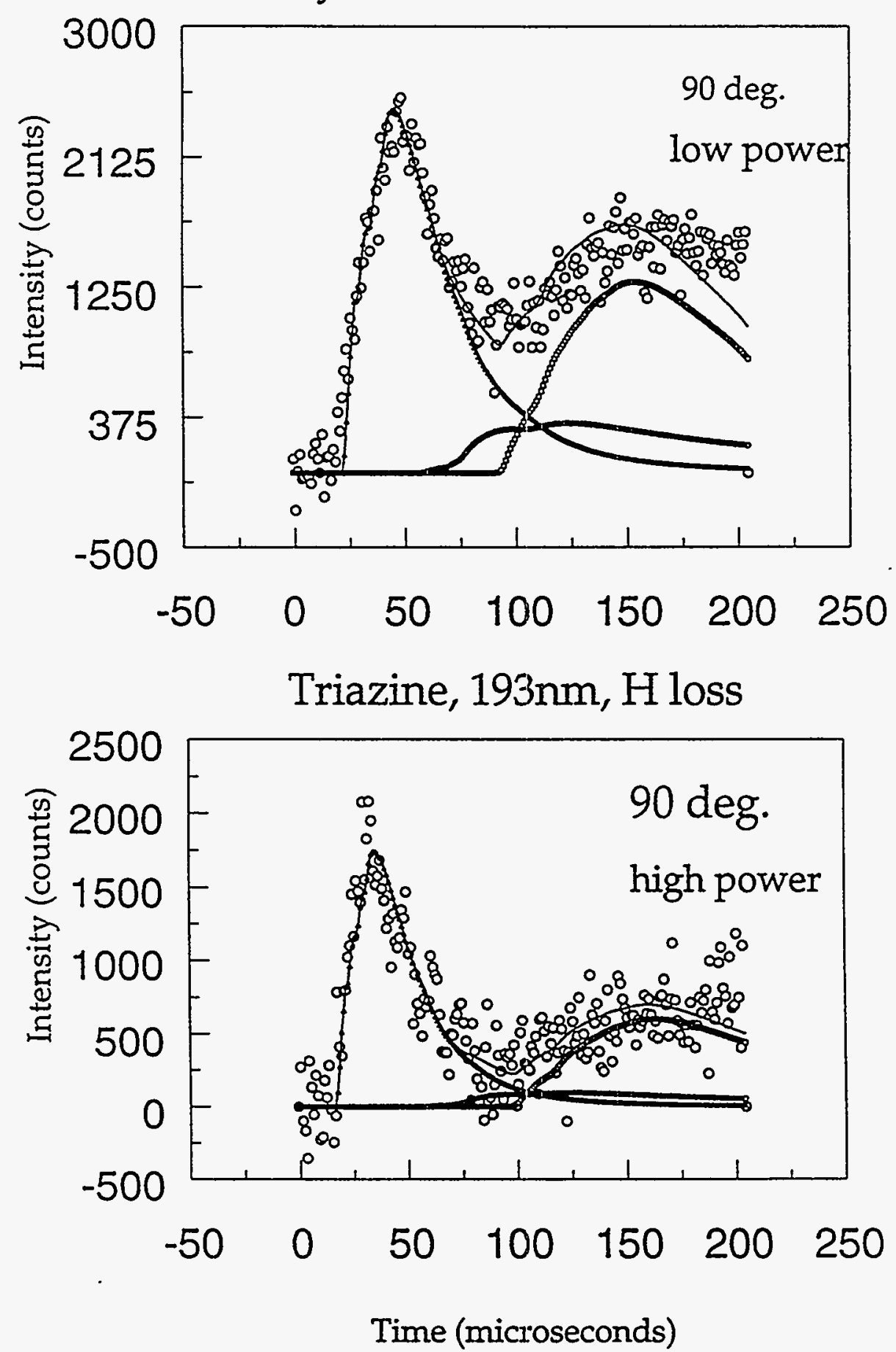

Figure 1-32 

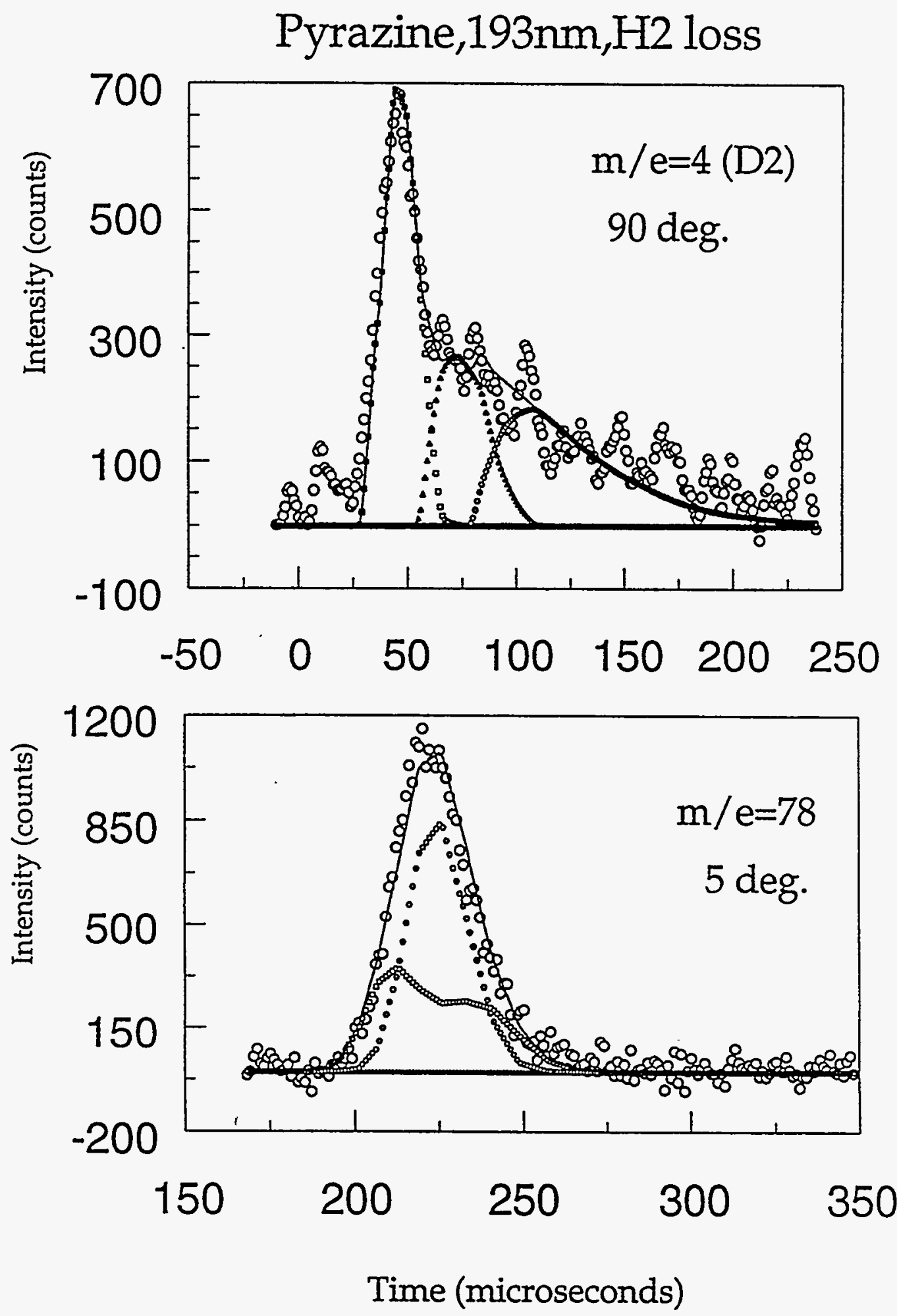

Figure 1-33 


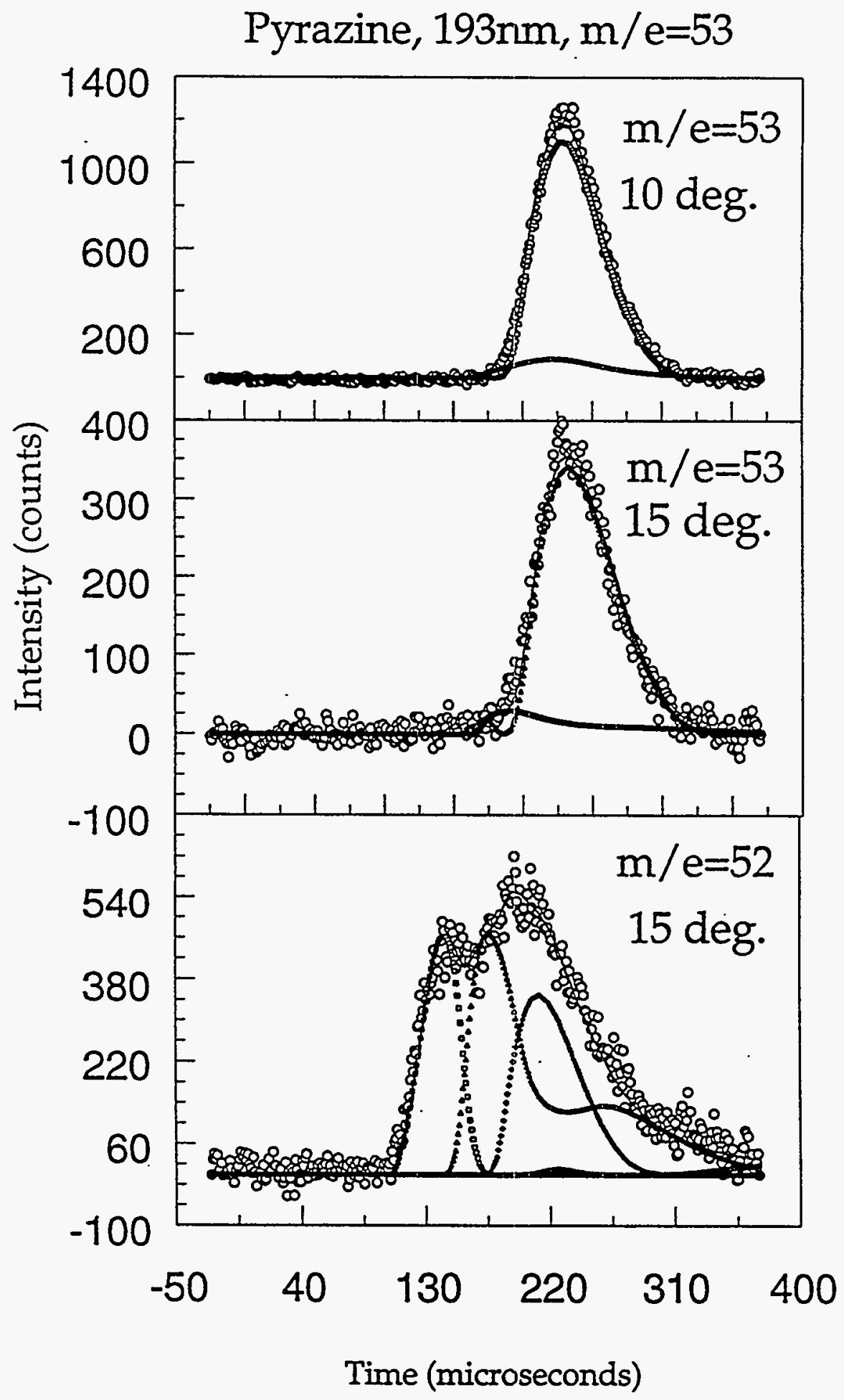

Figure 1-34 


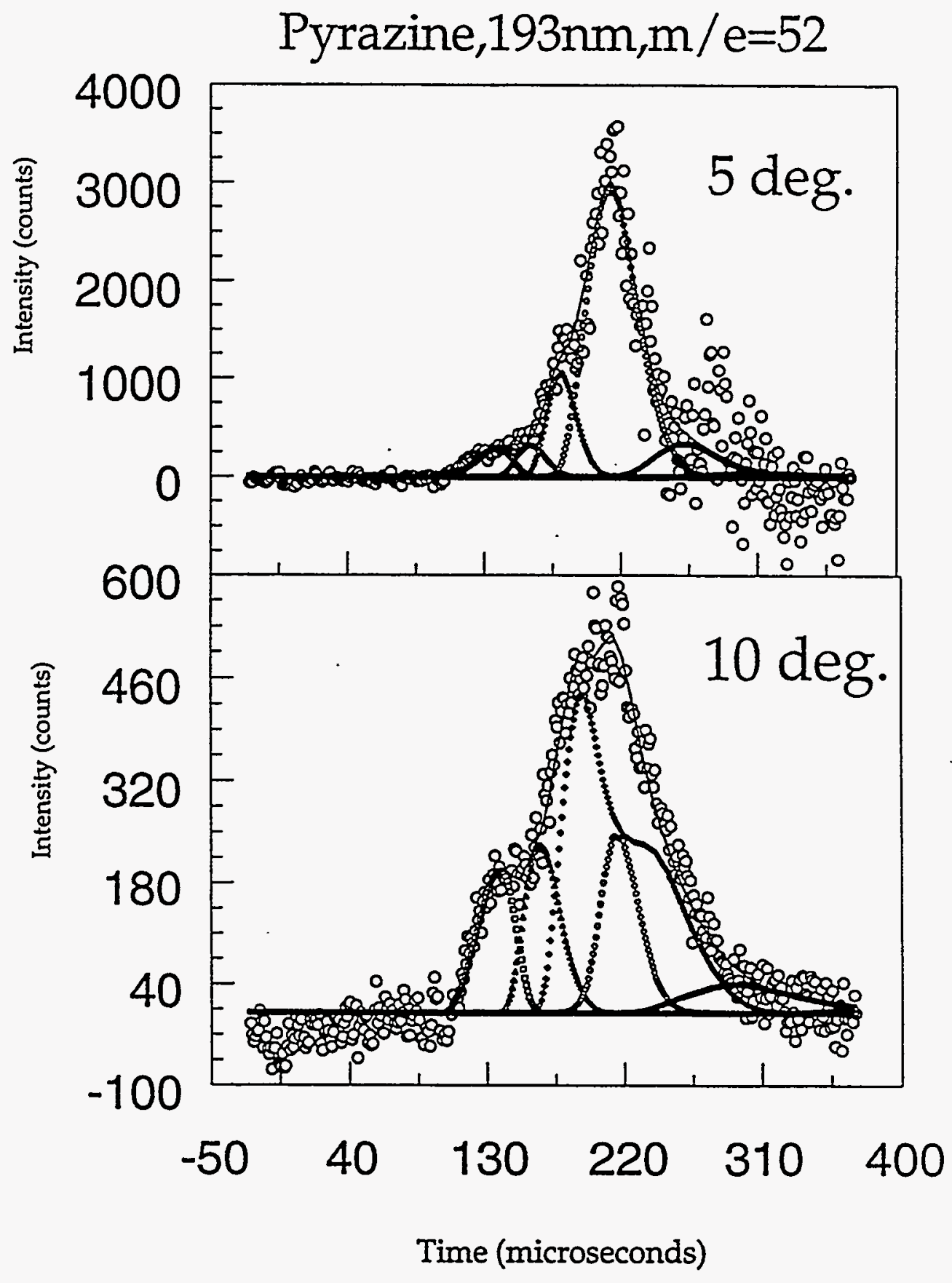

Figure 1-35 


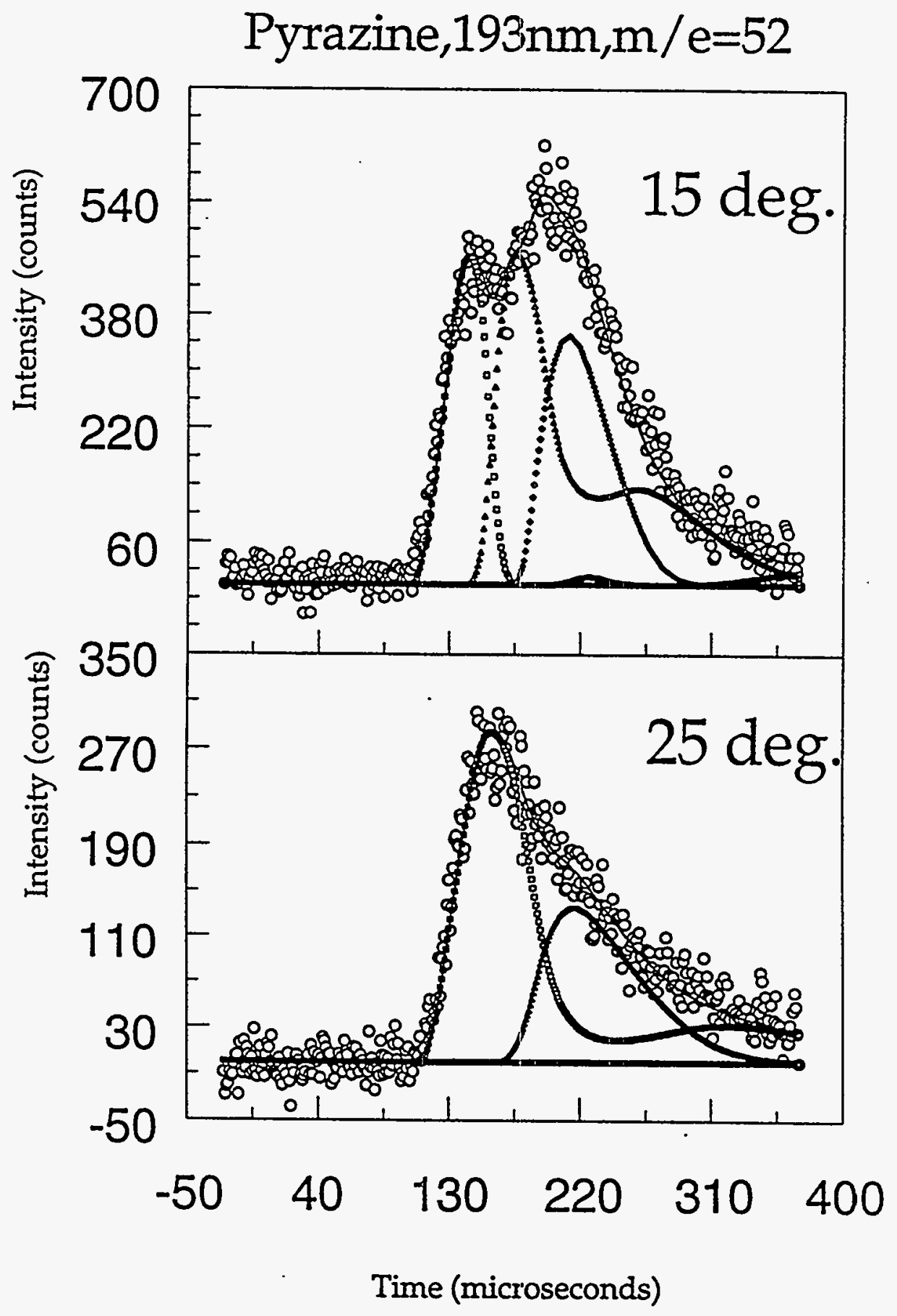

Figure 1-36 


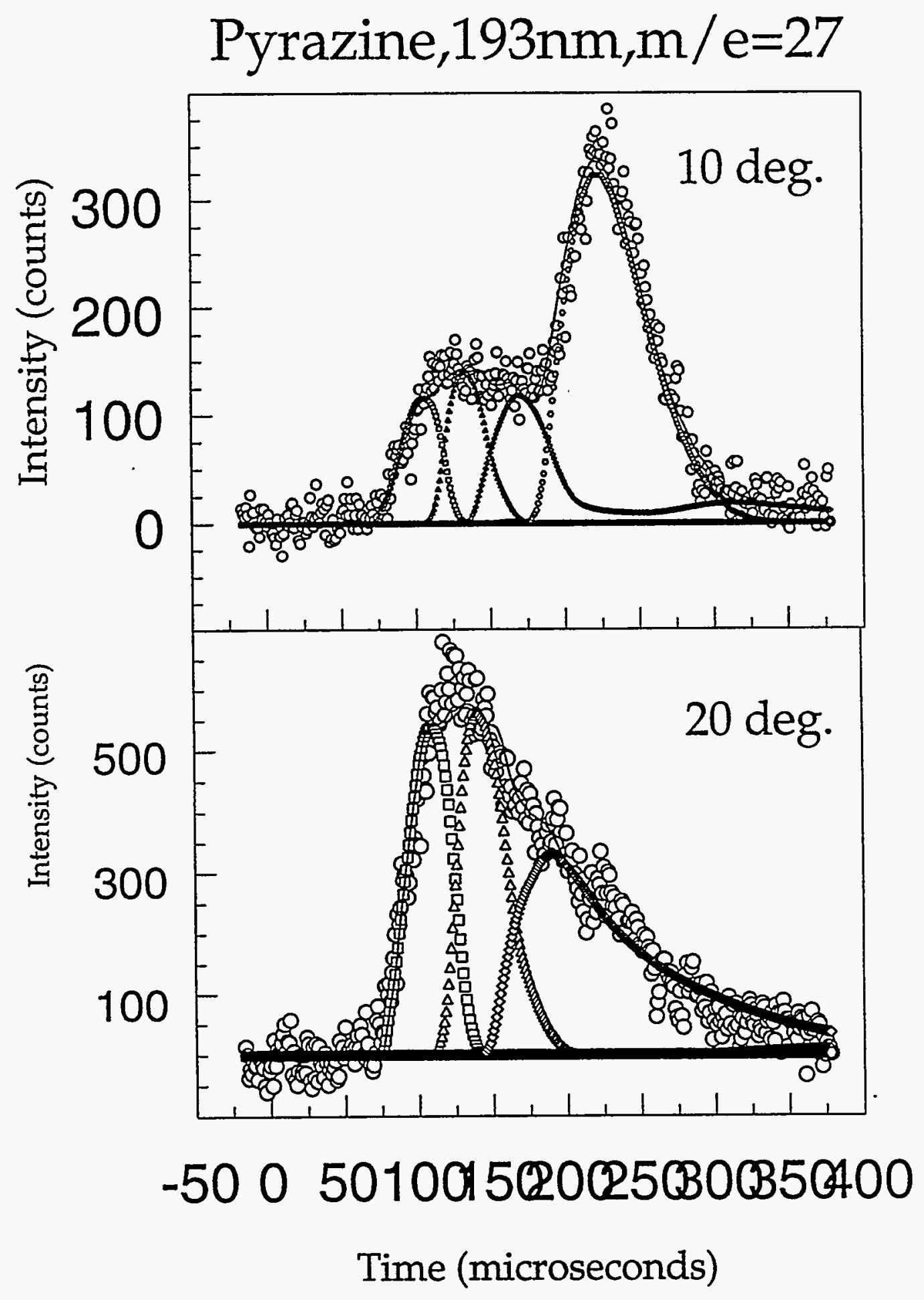

Figure 1-37 


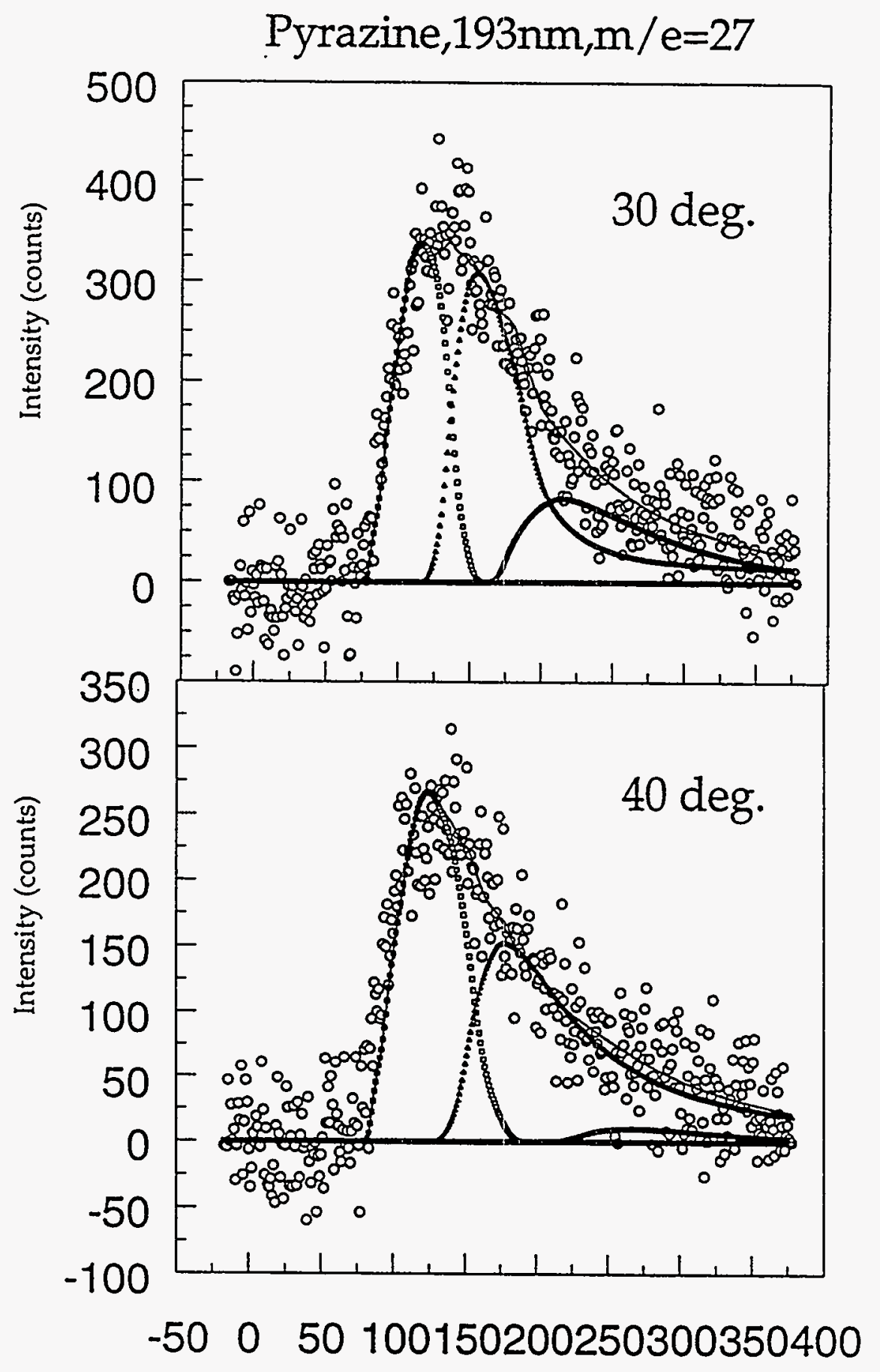

Time (microseconds)

Figure 1-38 
Pyrazine,193nm,m/e=26

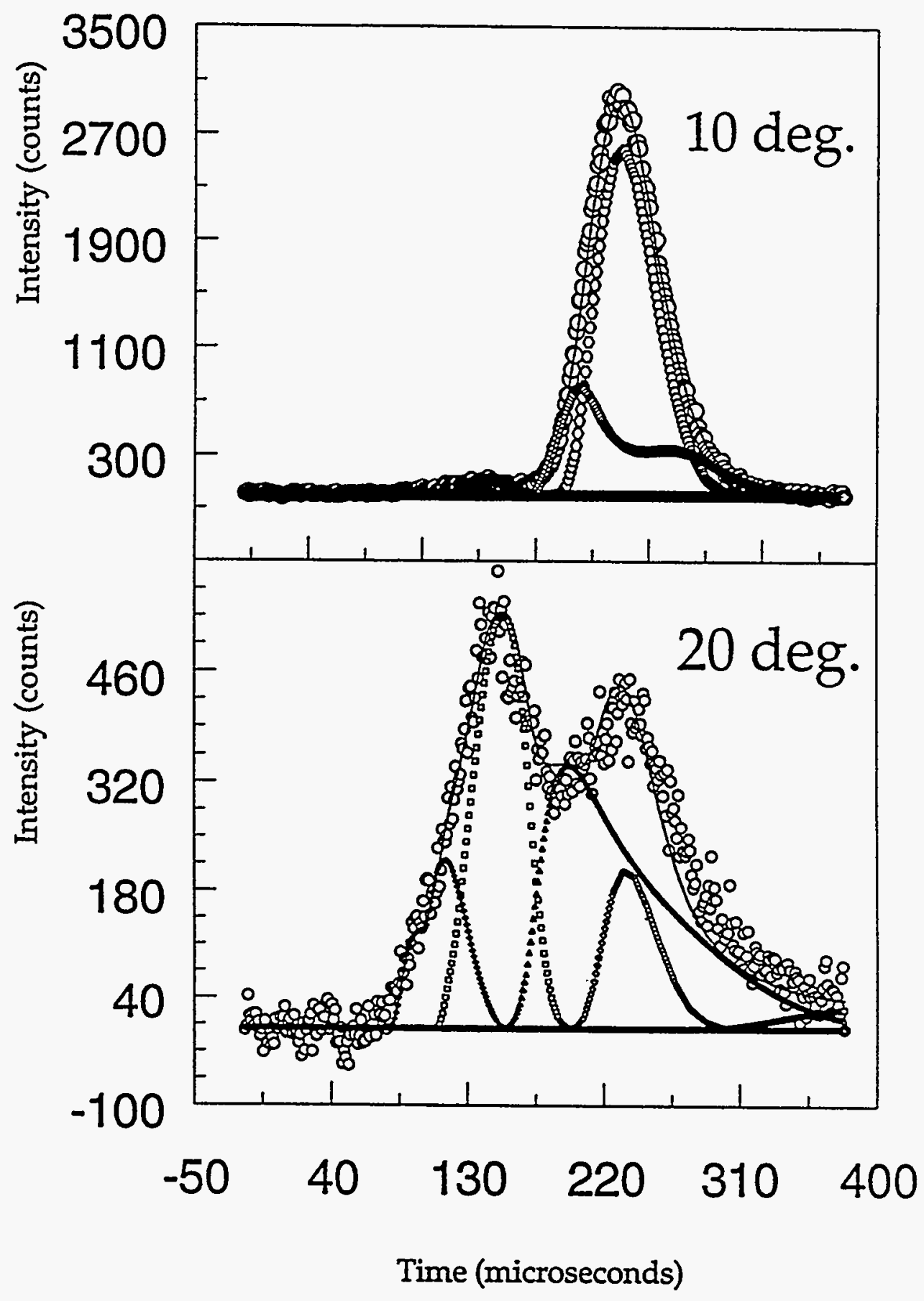

Figure 1-39 


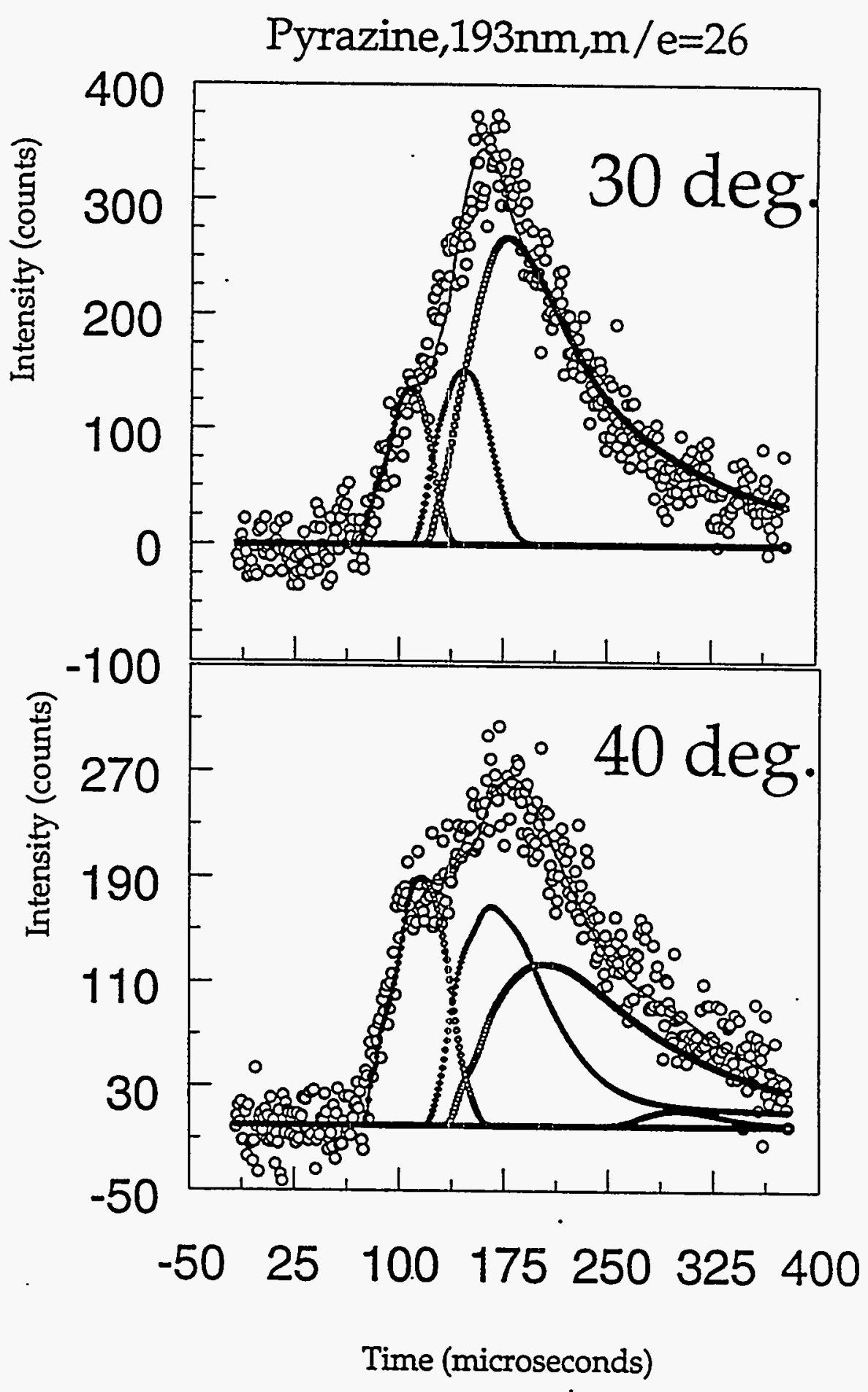

Figure 1-40 
Pyrazine,193nm, C2H2 loss

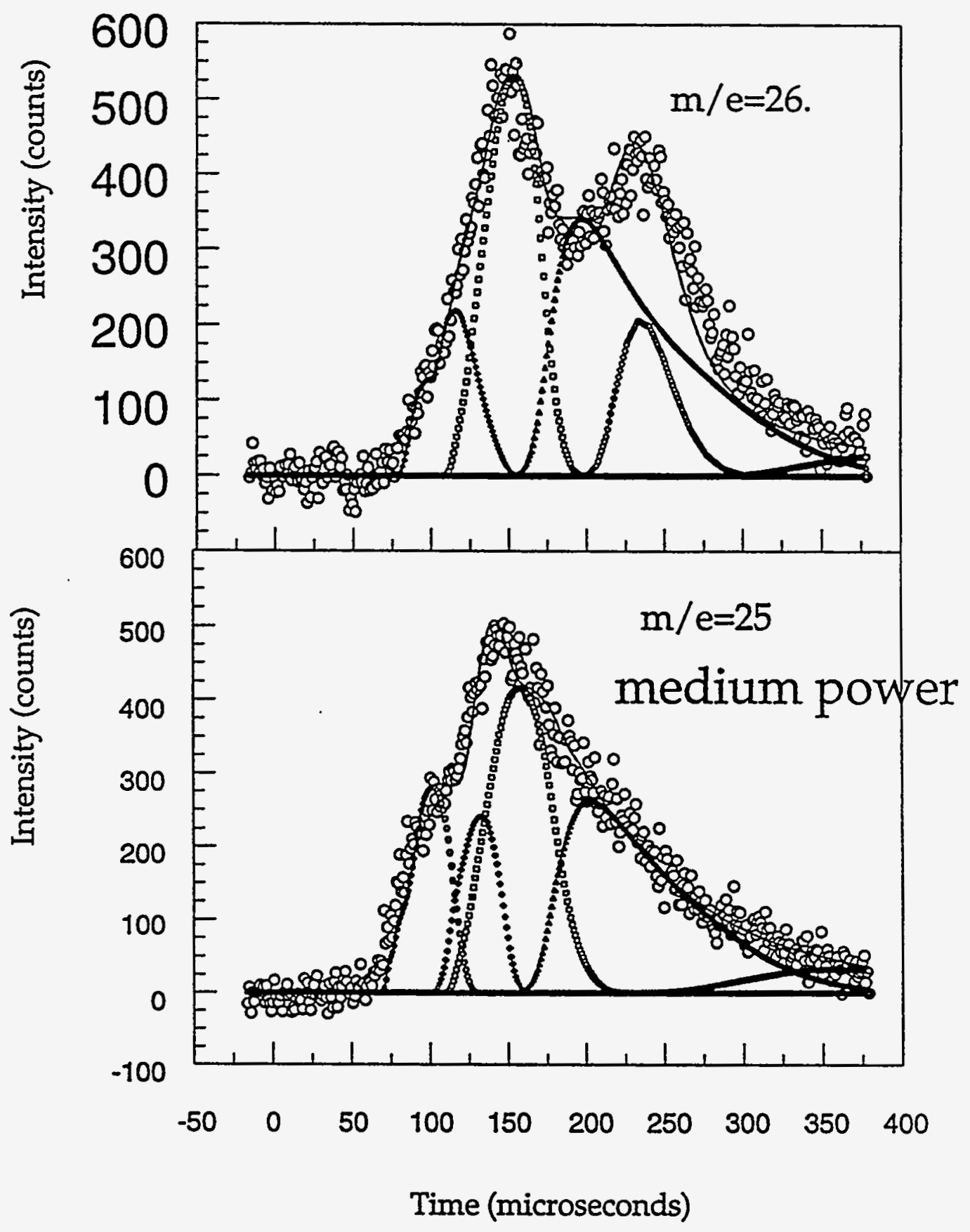

Figure 1-41 


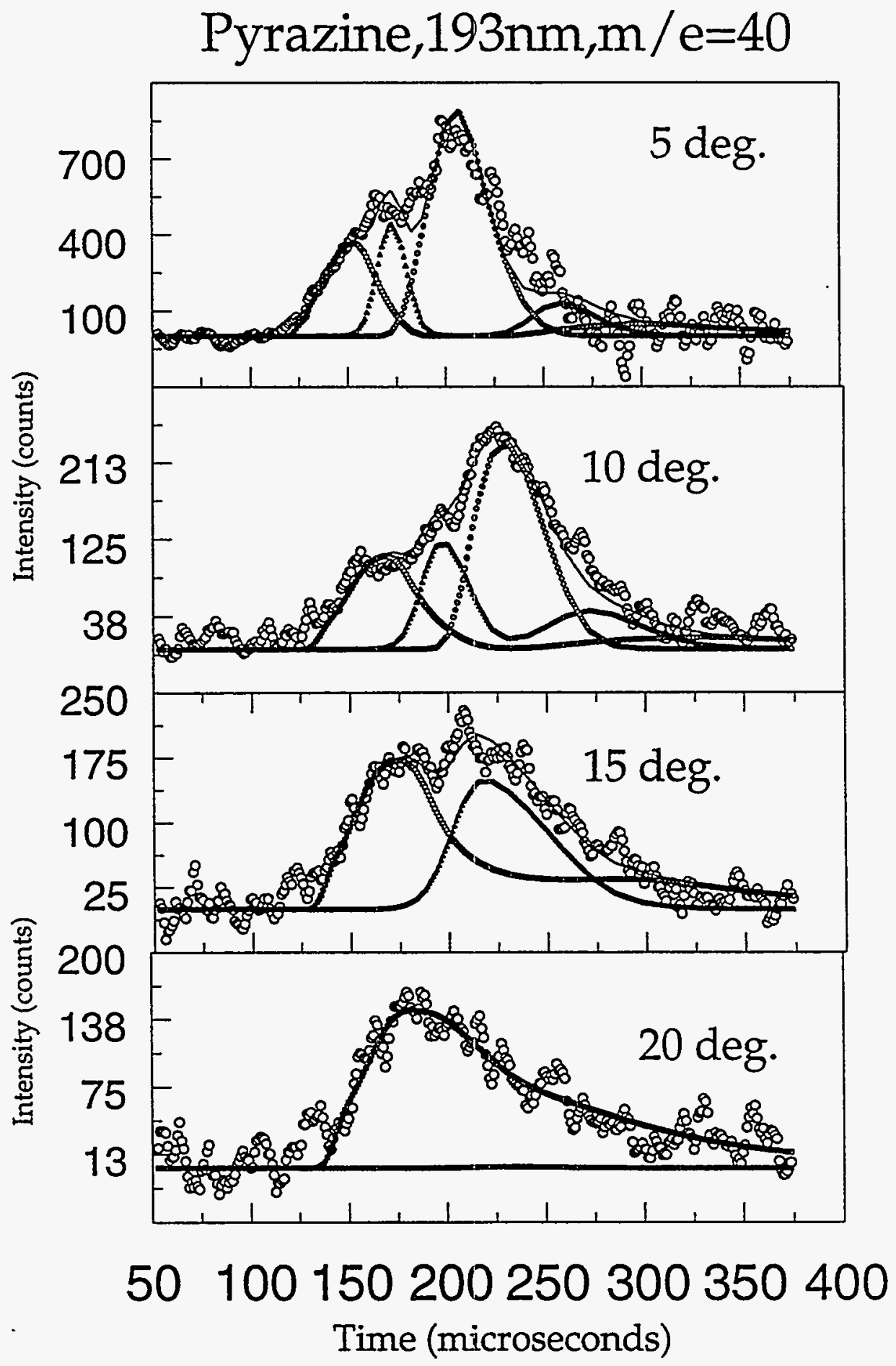

Figure 1-42 

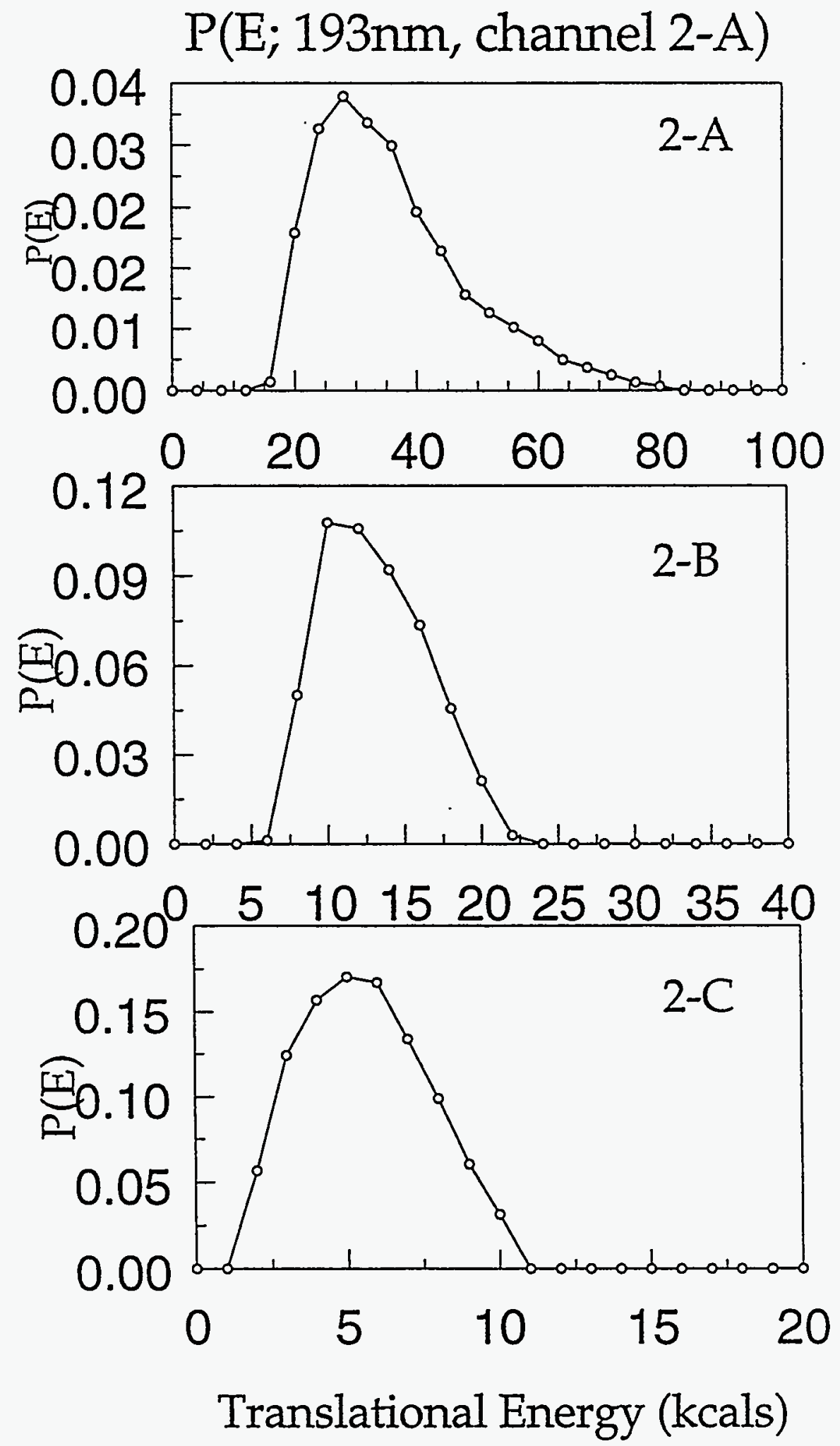

Figure 1-43 

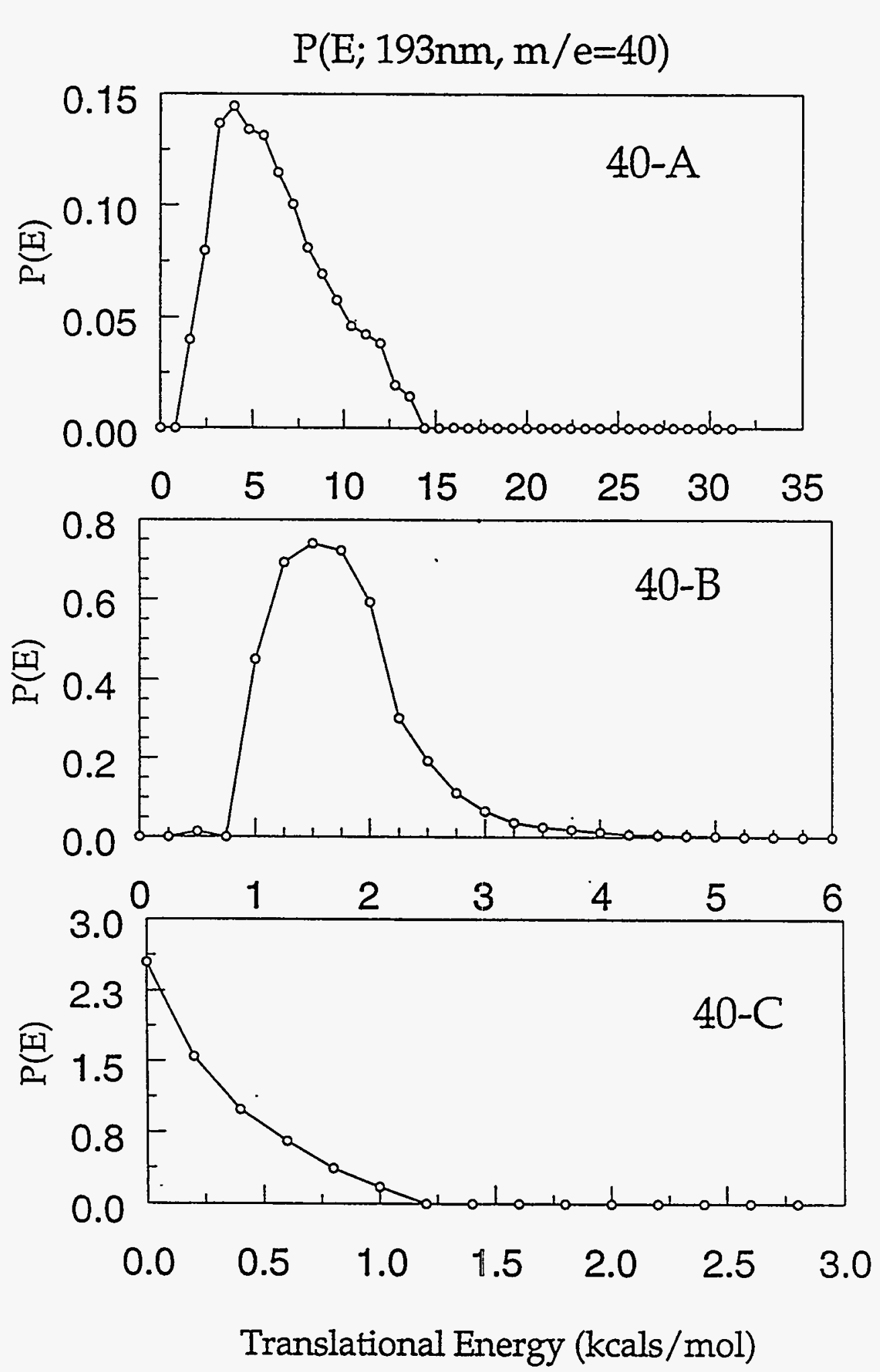

Figure 1-44 

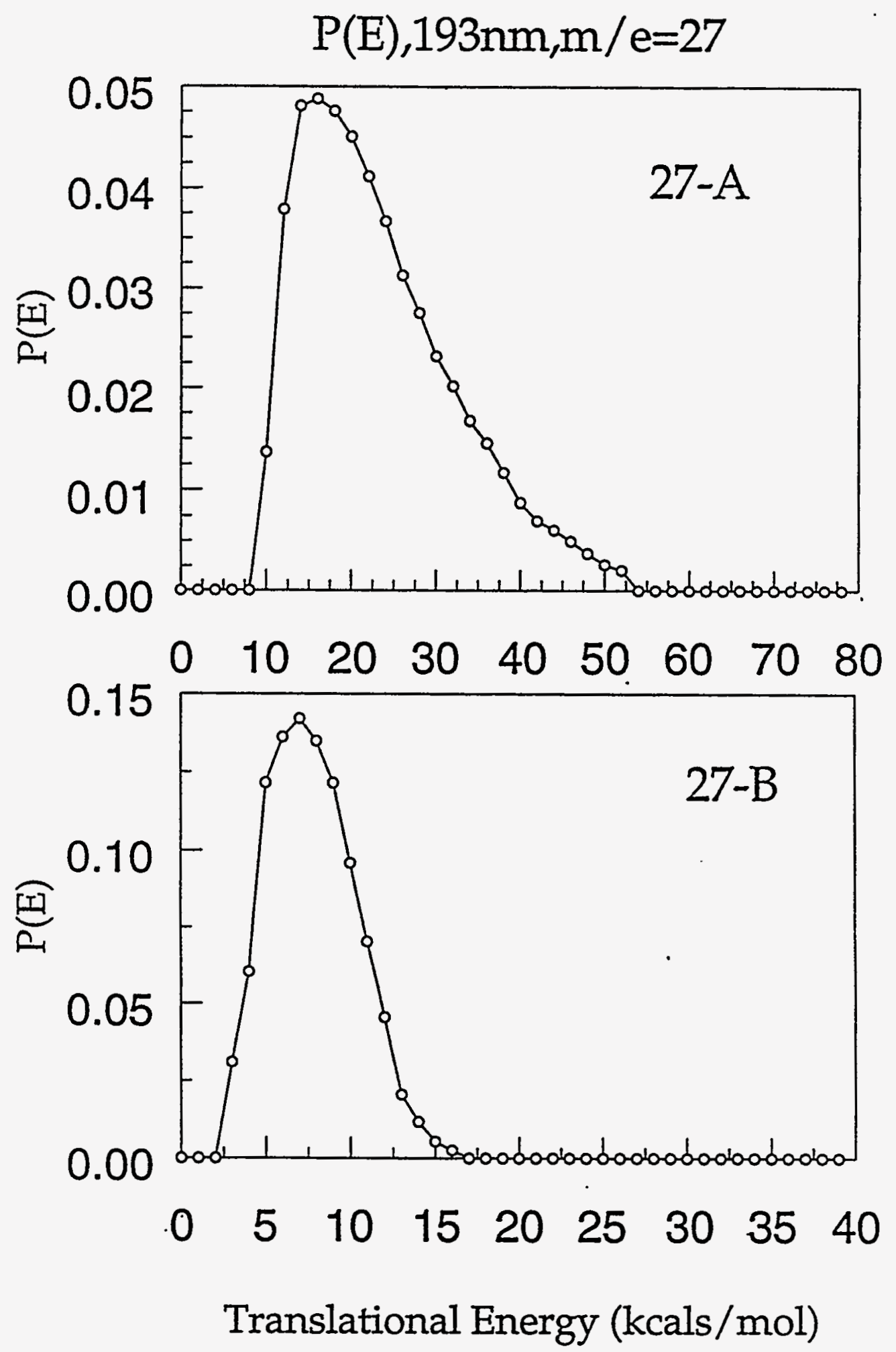

Figure 1-45 

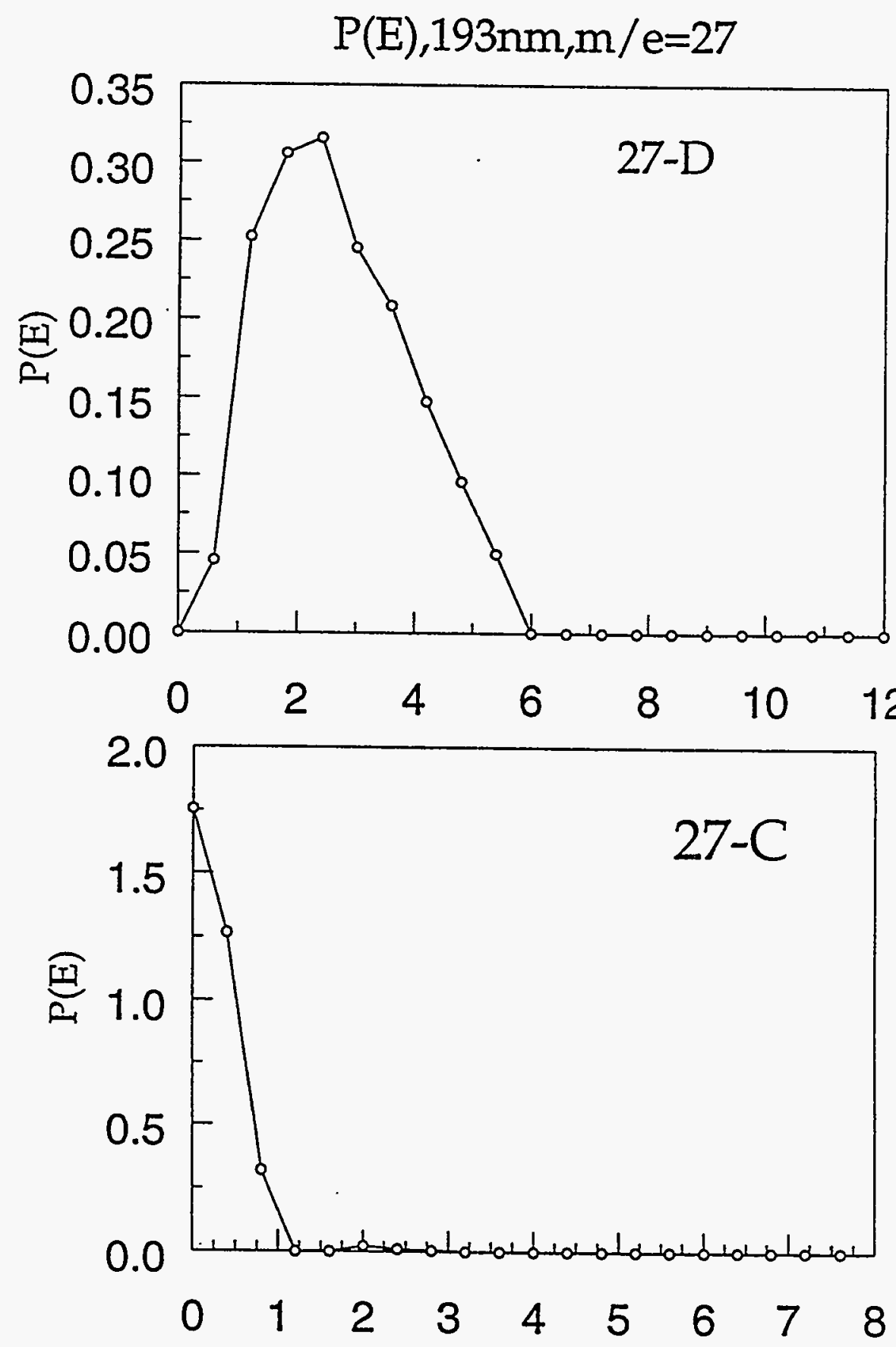

Translational Energy (kcals/mol)

Figure 1-46 


\section{Energy Level Diagram (with Standard Heats of Formation)}

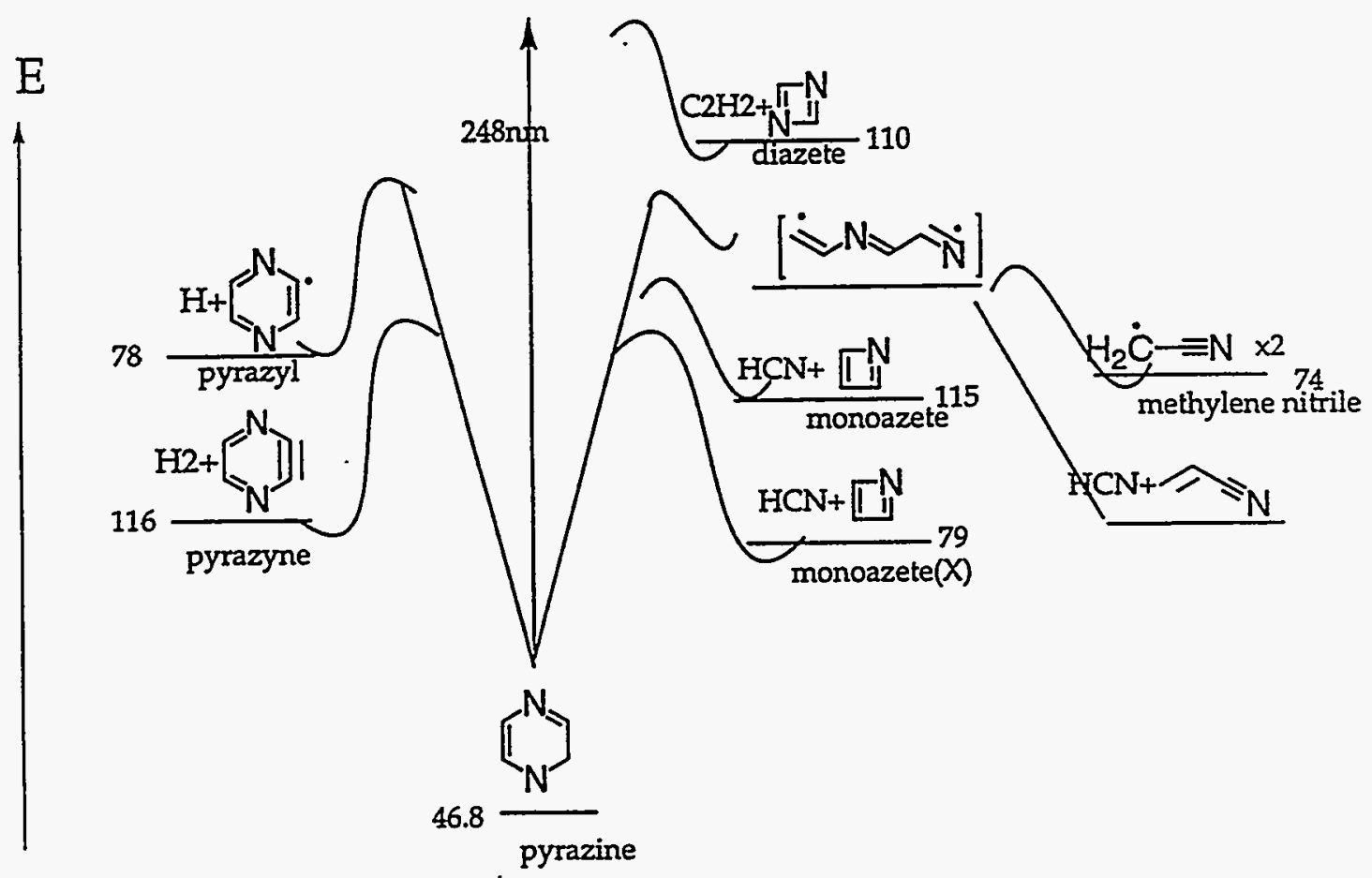

Values in $\mathrm{kcal} / \mathrm{mol}$, referring to the named species

Figure 1-47 
Triazine, $248 \mathrm{~nm}, \mathrm{H}$ loss

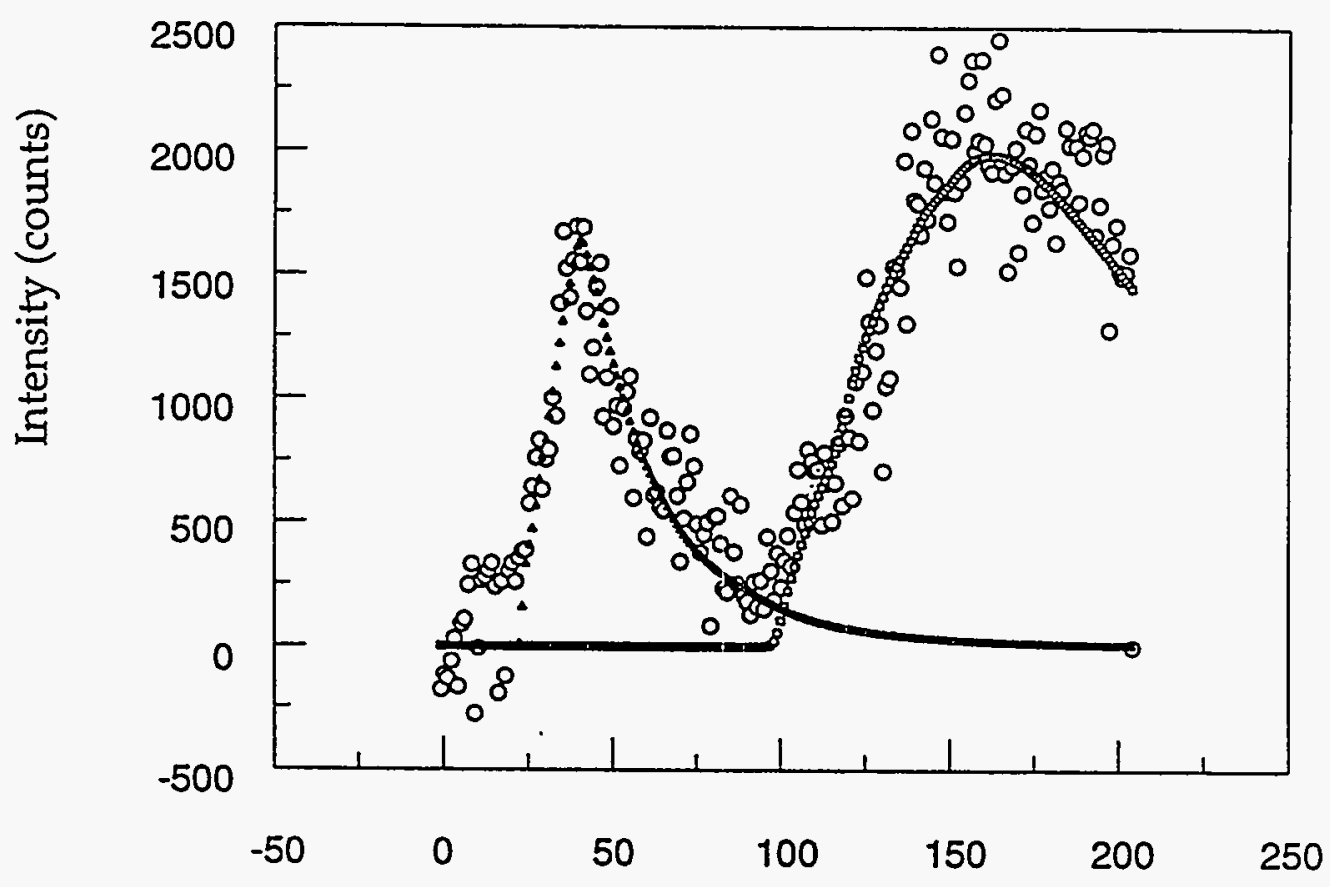

Time (microseconds)

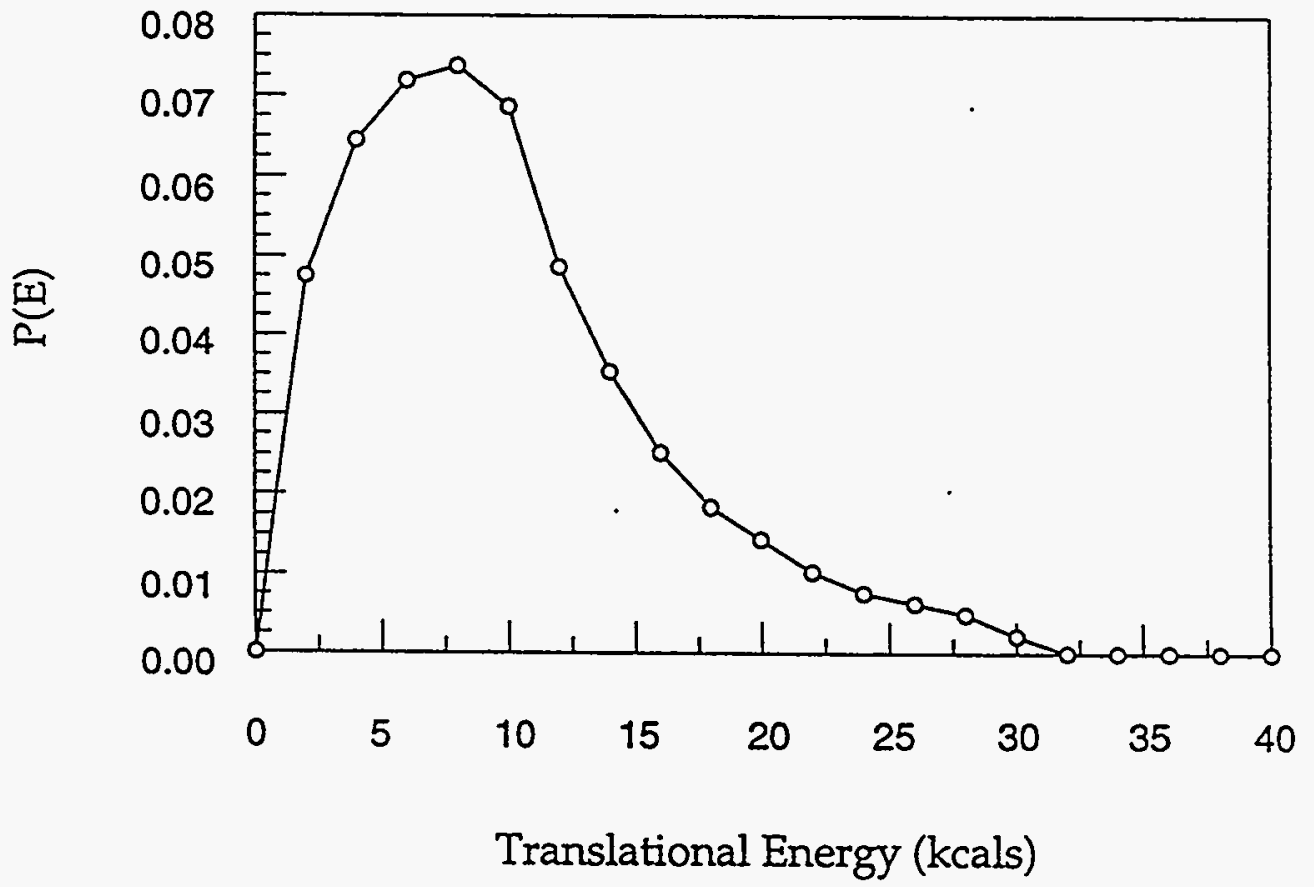

Figure 1-48 
Triazine, $248 \mathrm{~nm}$

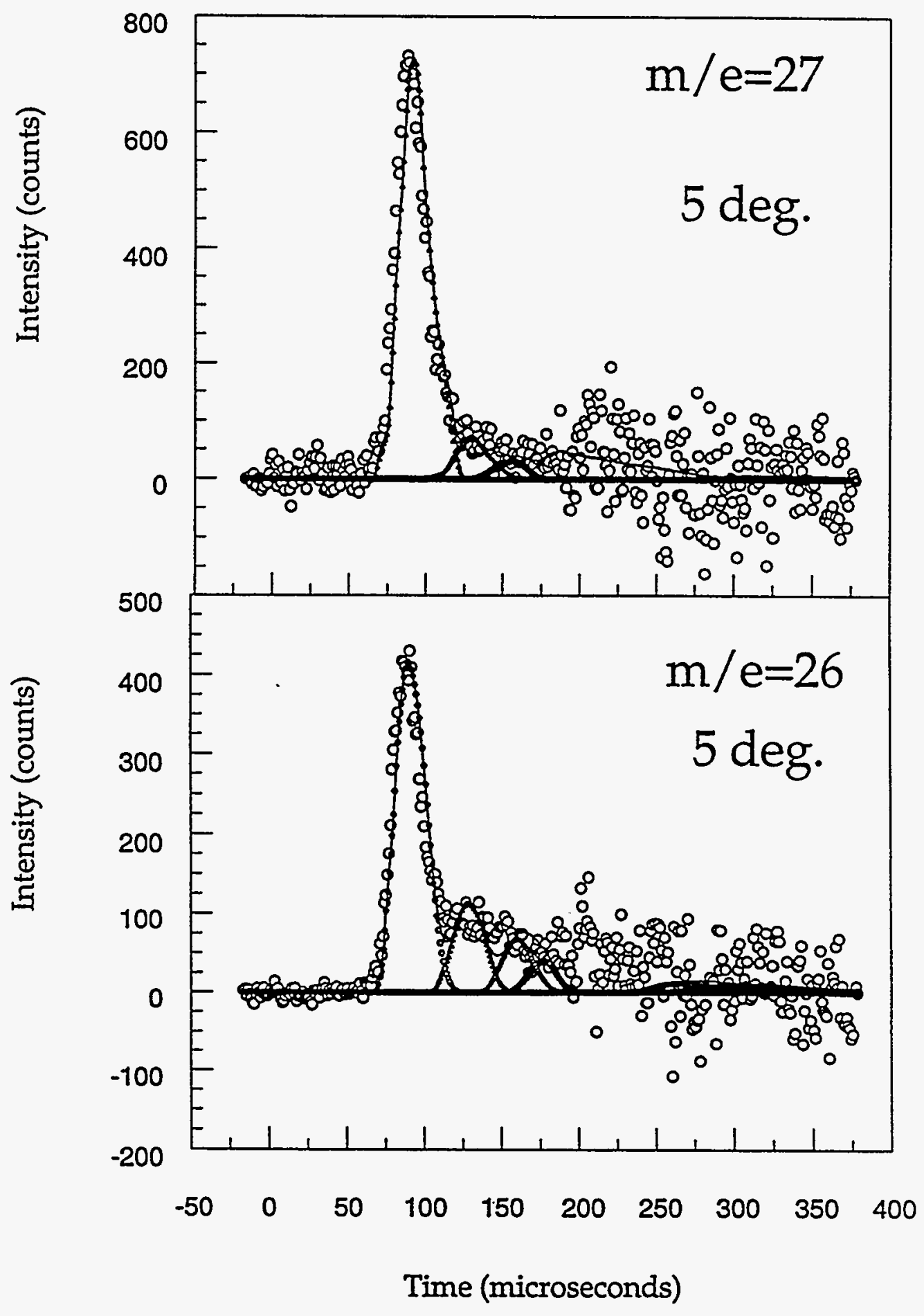

Figure 1-49 
Triazine, $248 \mathrm{~nm}$

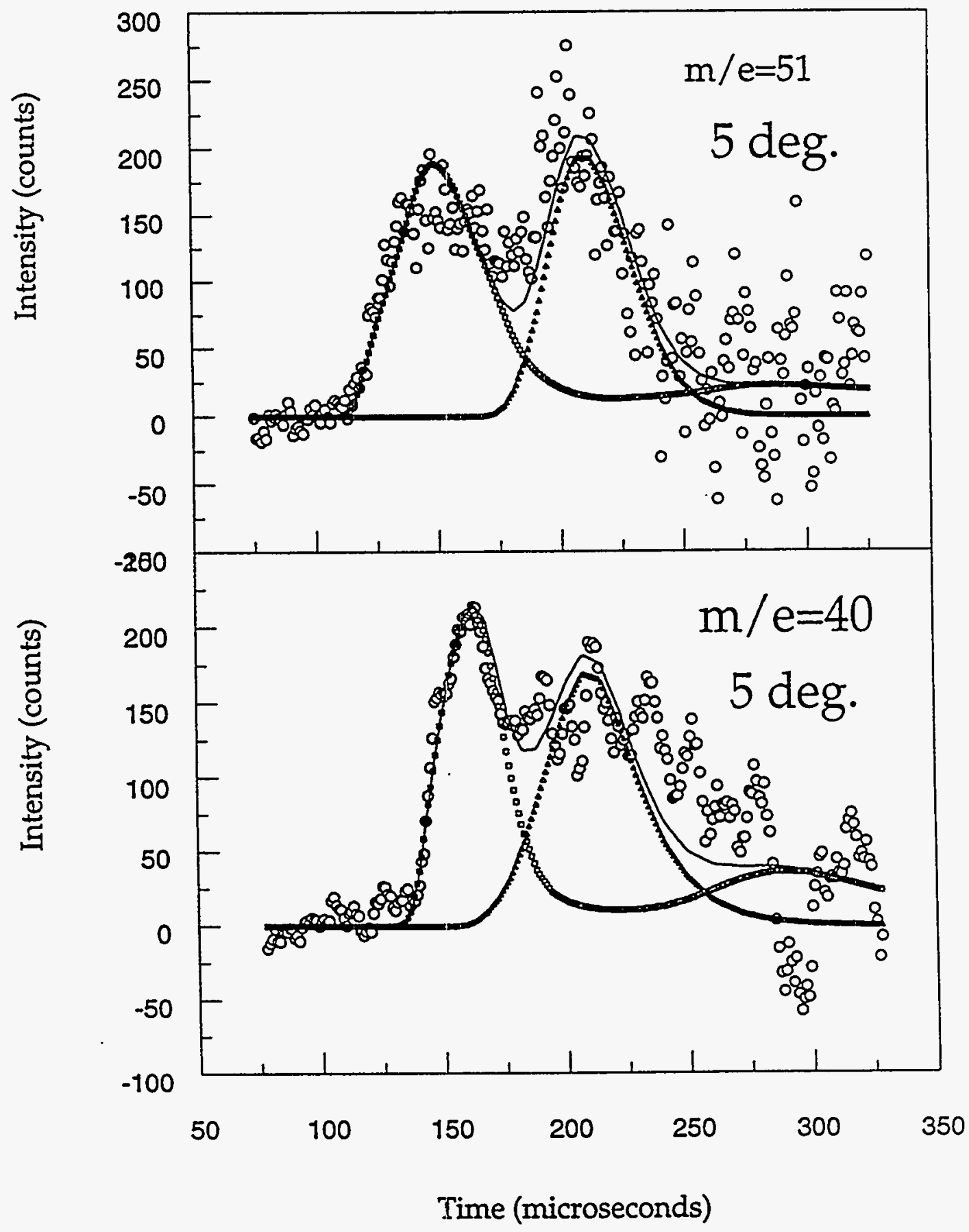

Figure 1-50 

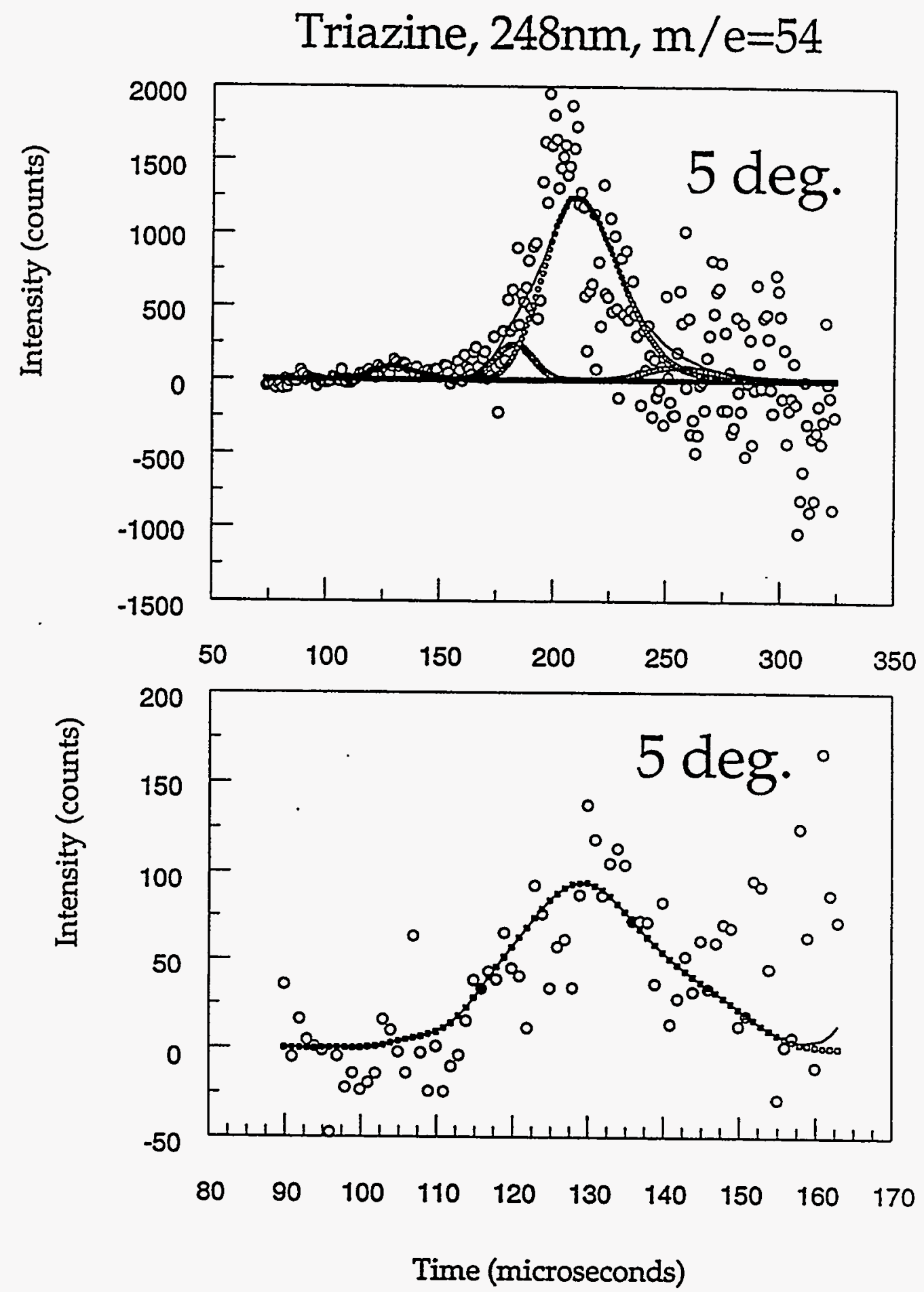

Figure 1-51 


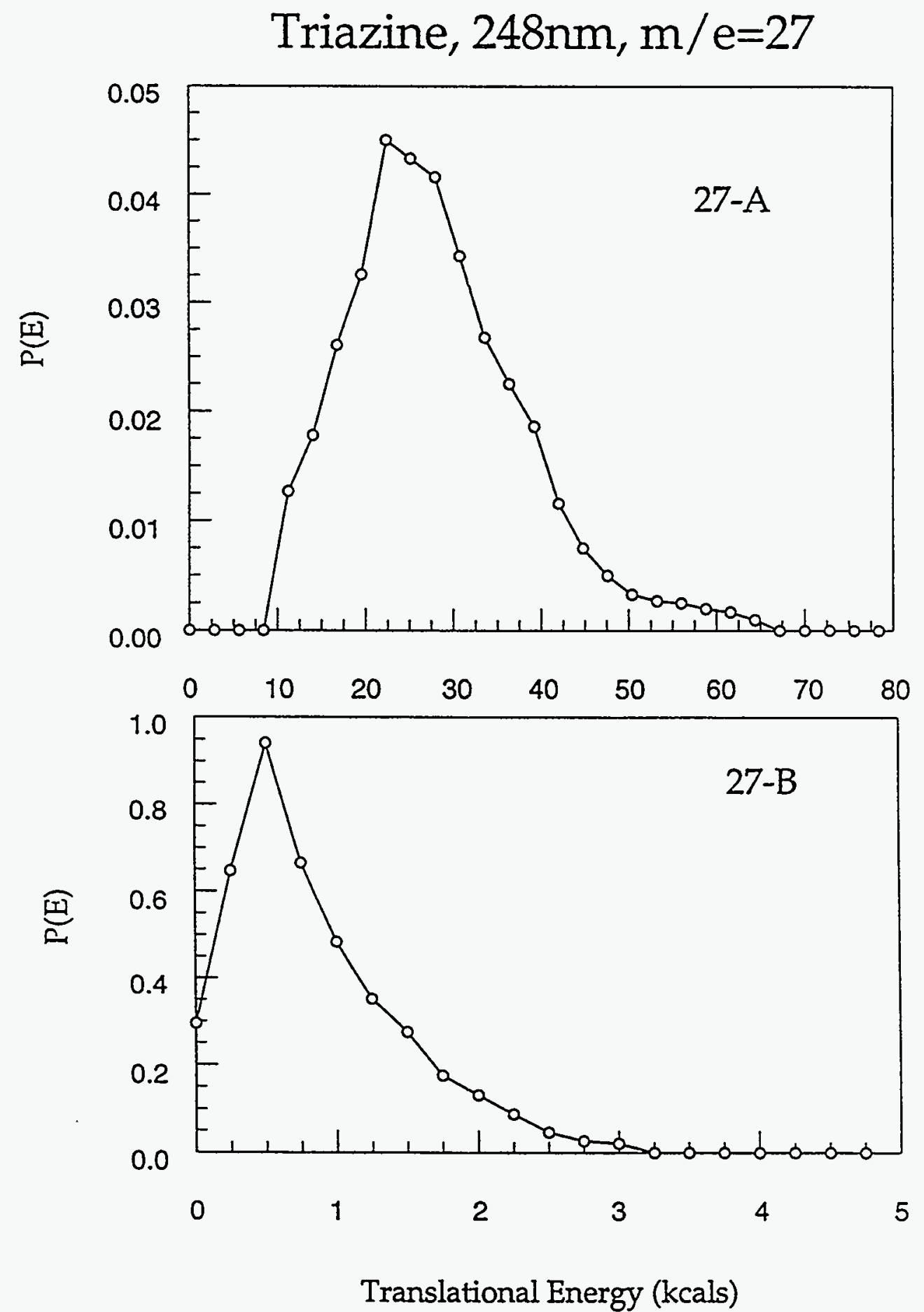

Figure 1-52 

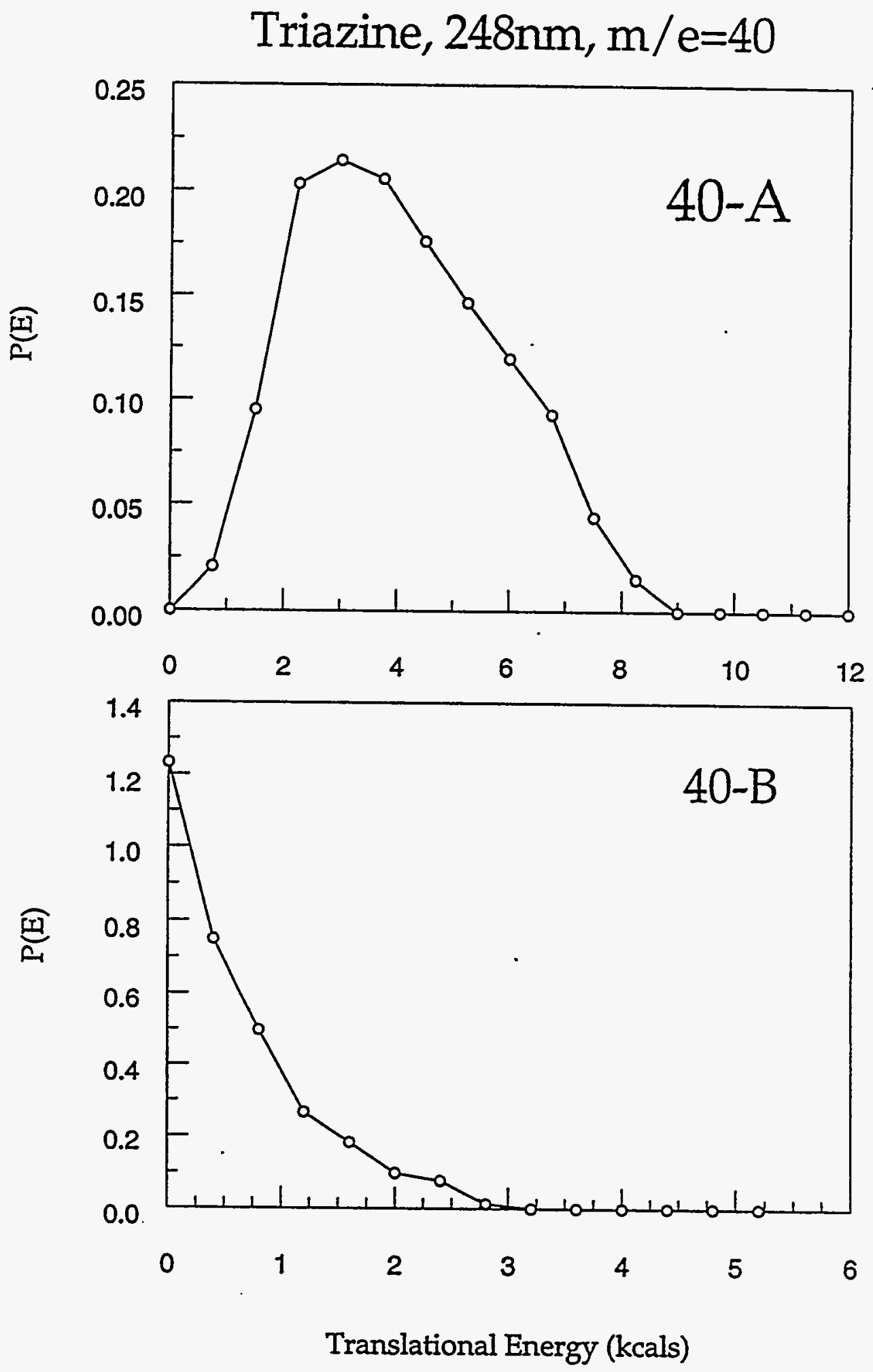

Figure 1-53 


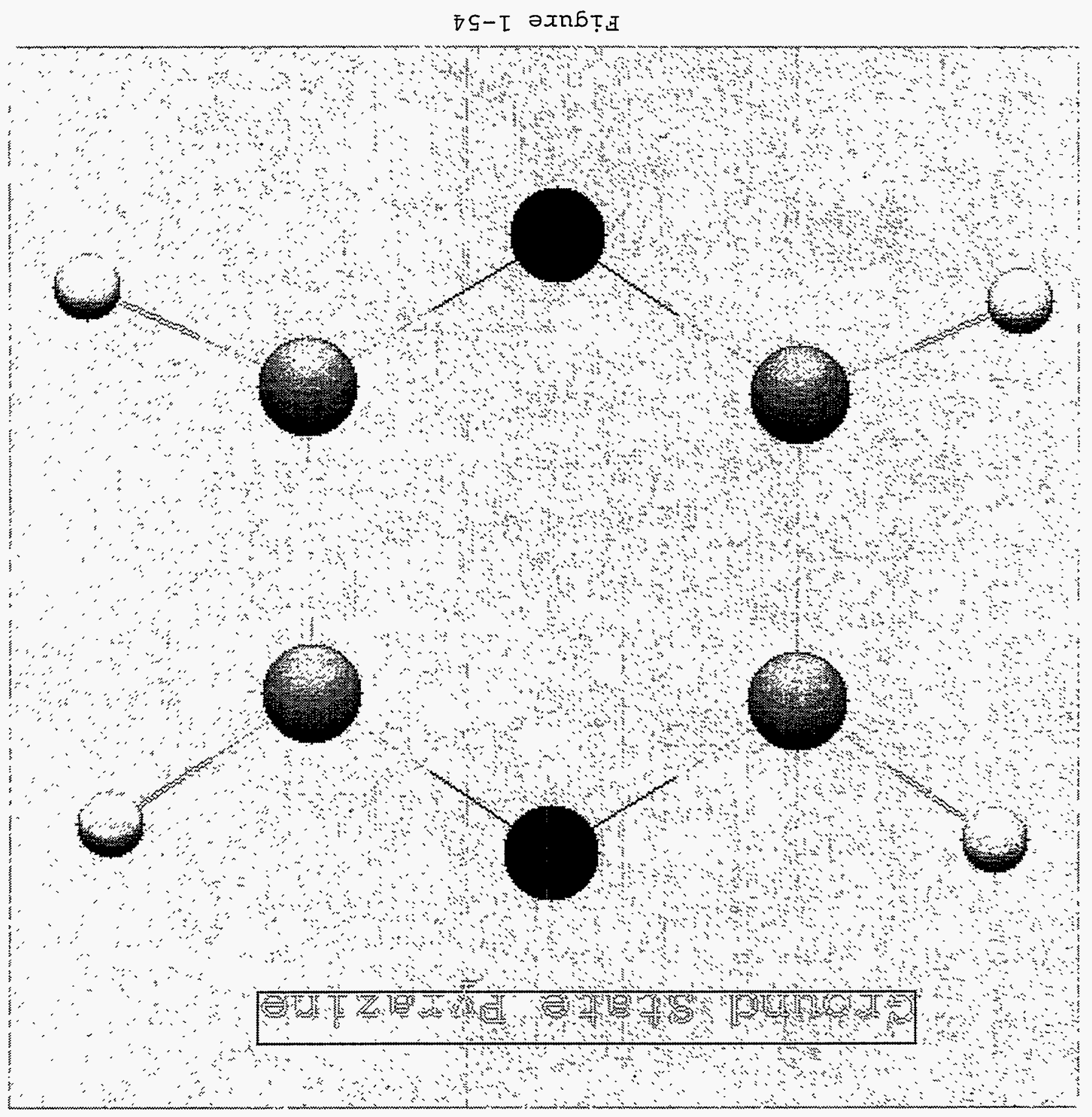




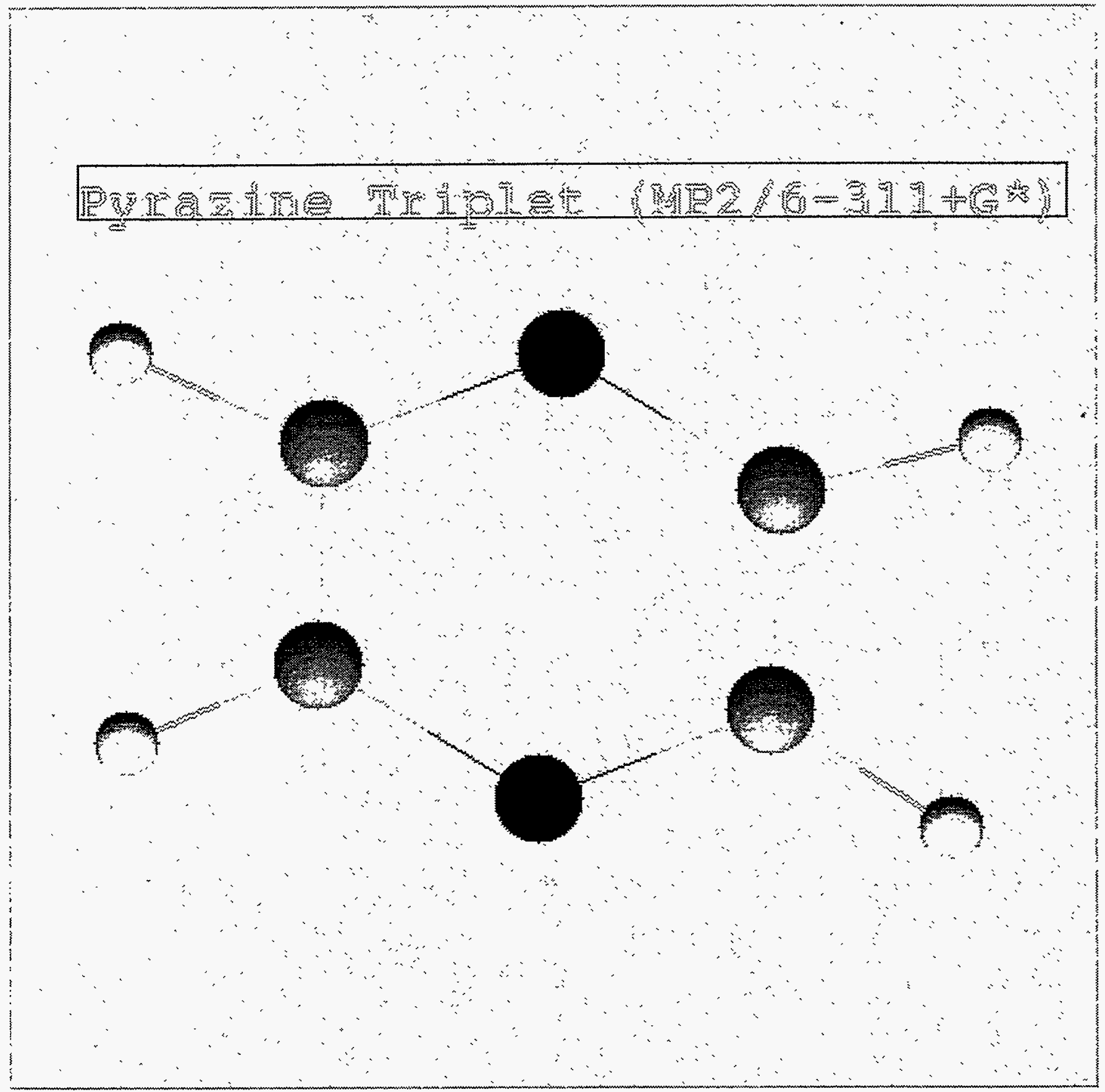

Figure 1-55 


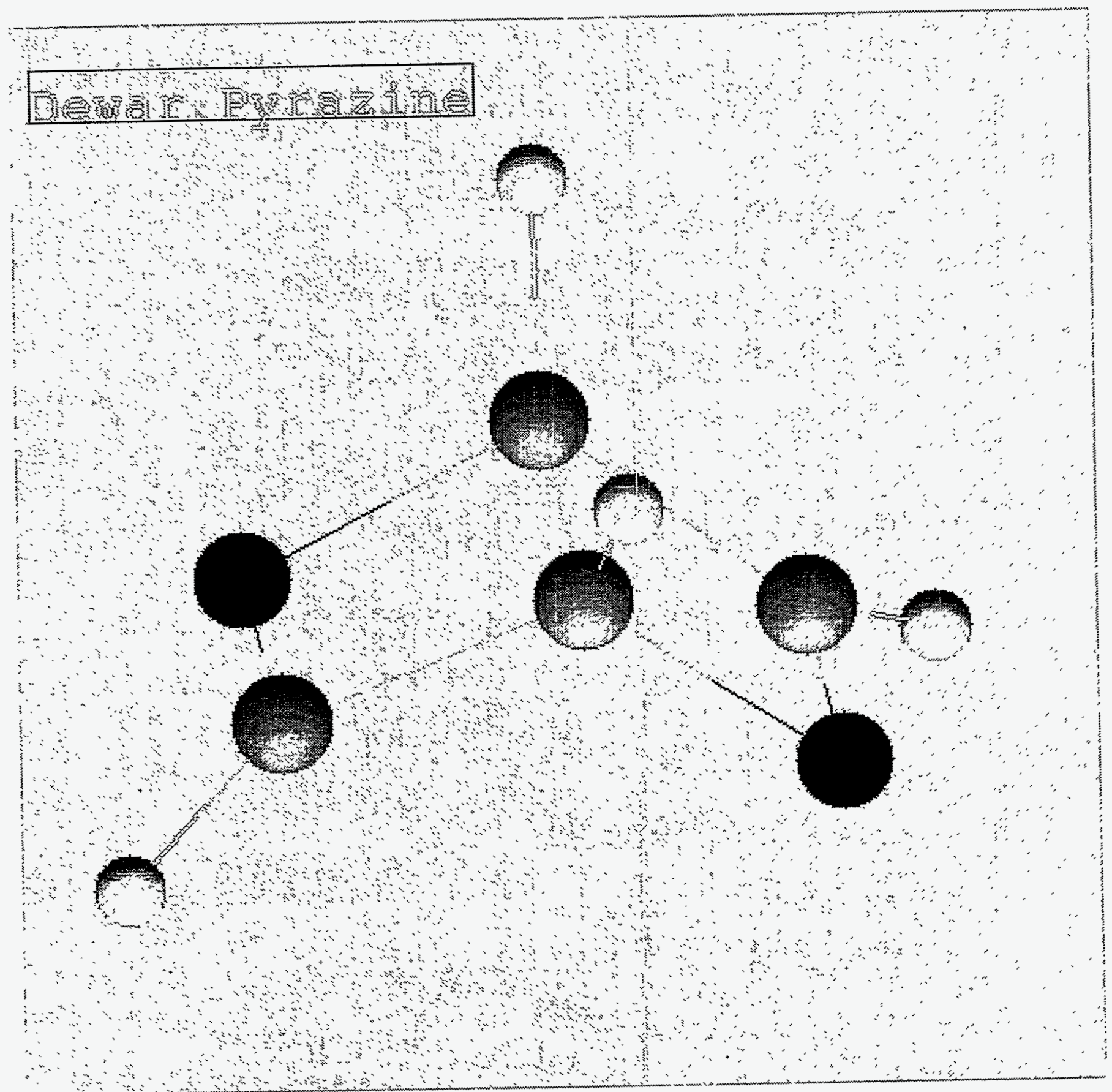

Figure 1-56 


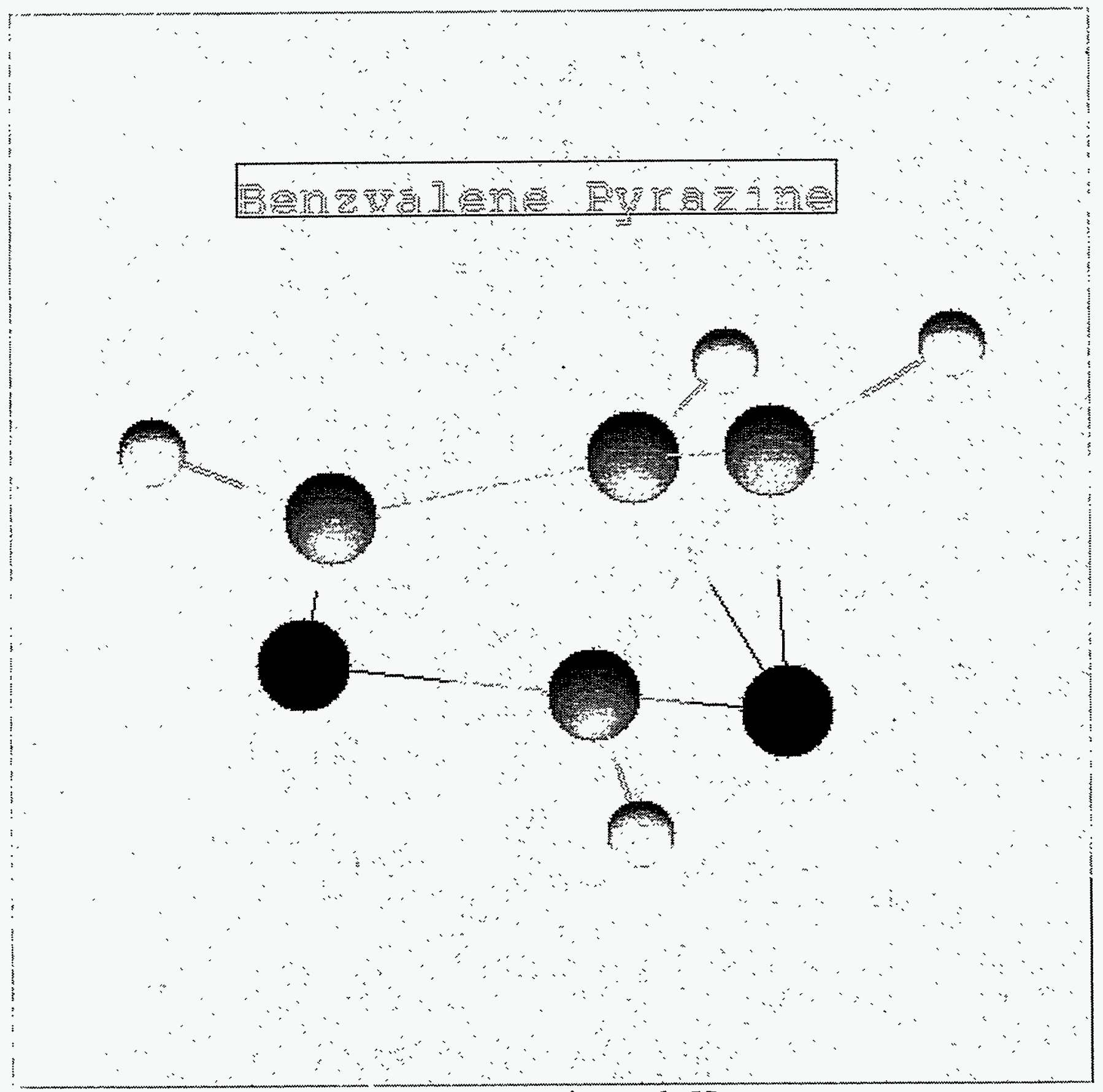

Figure 1-57 


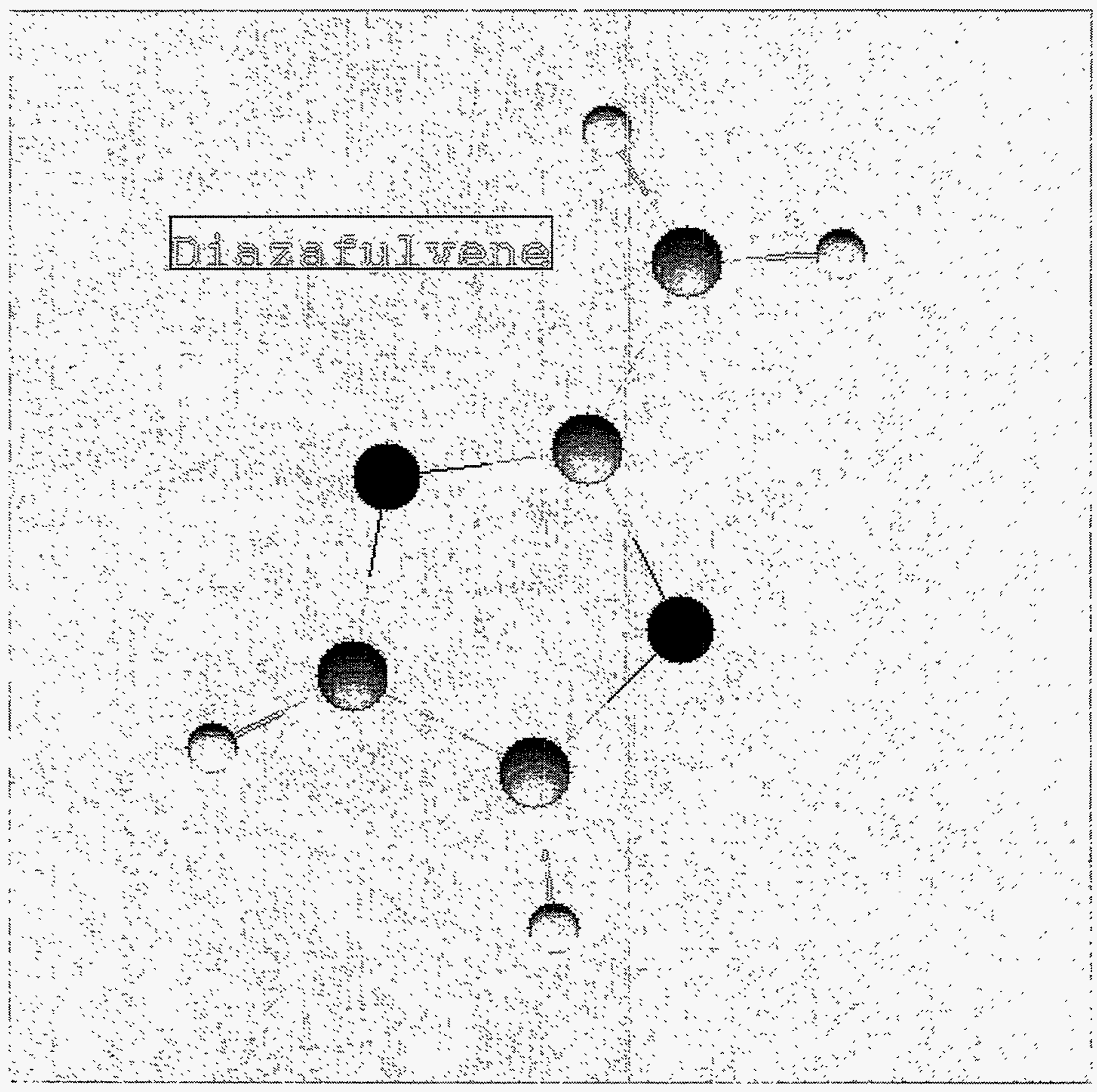

Figure 1-58 


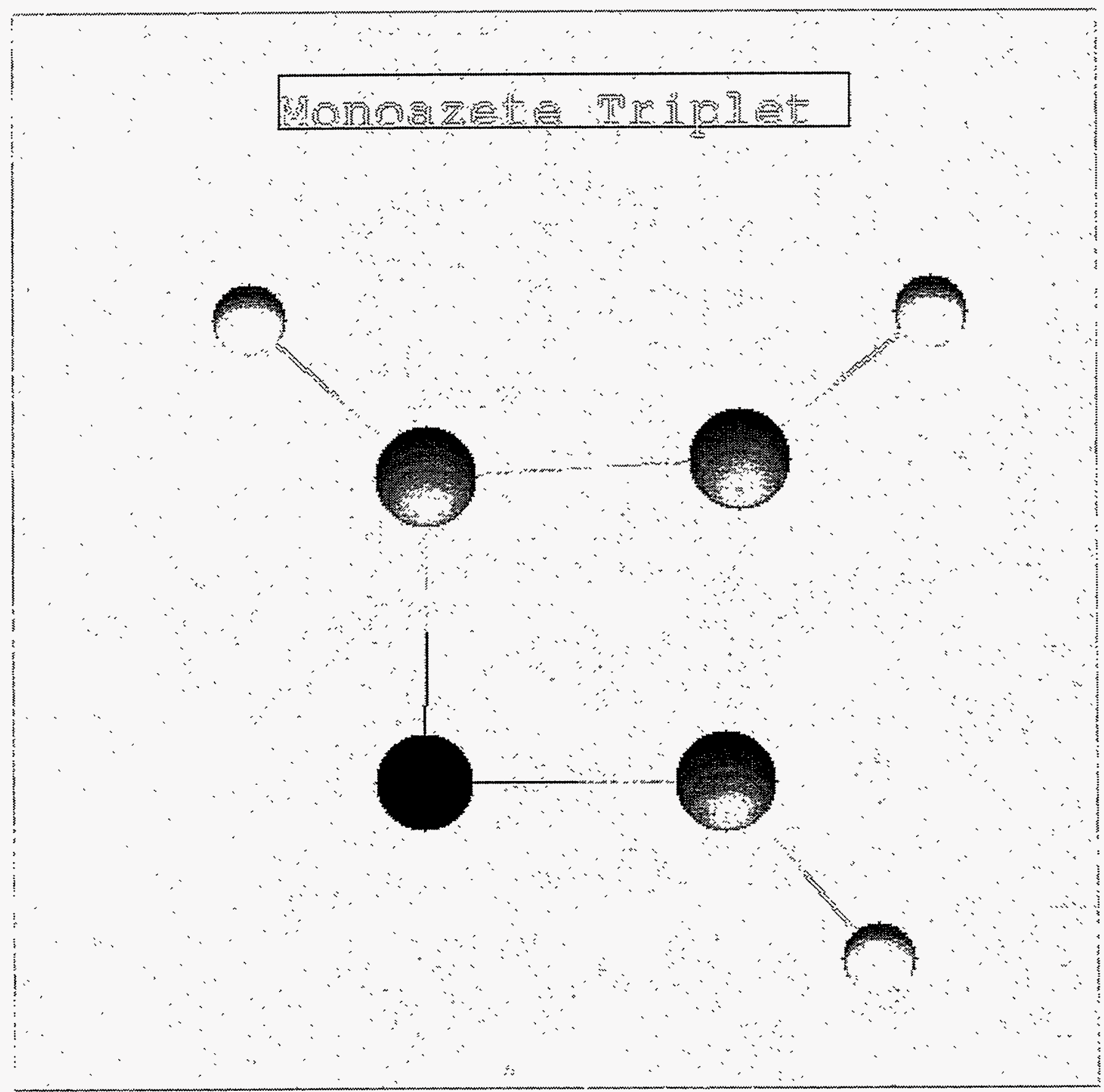

Figure 1-59 


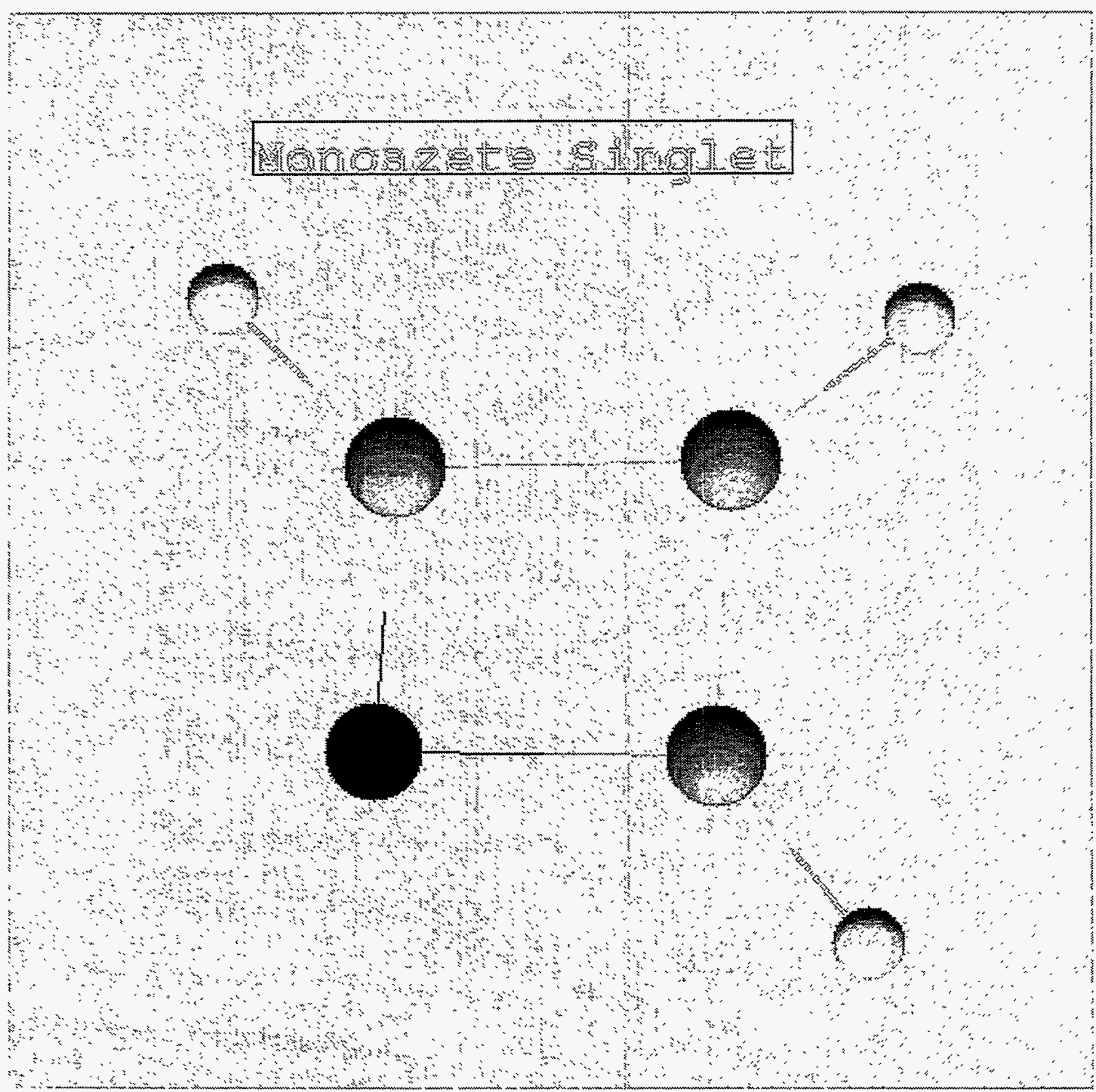

Figure 1-60 


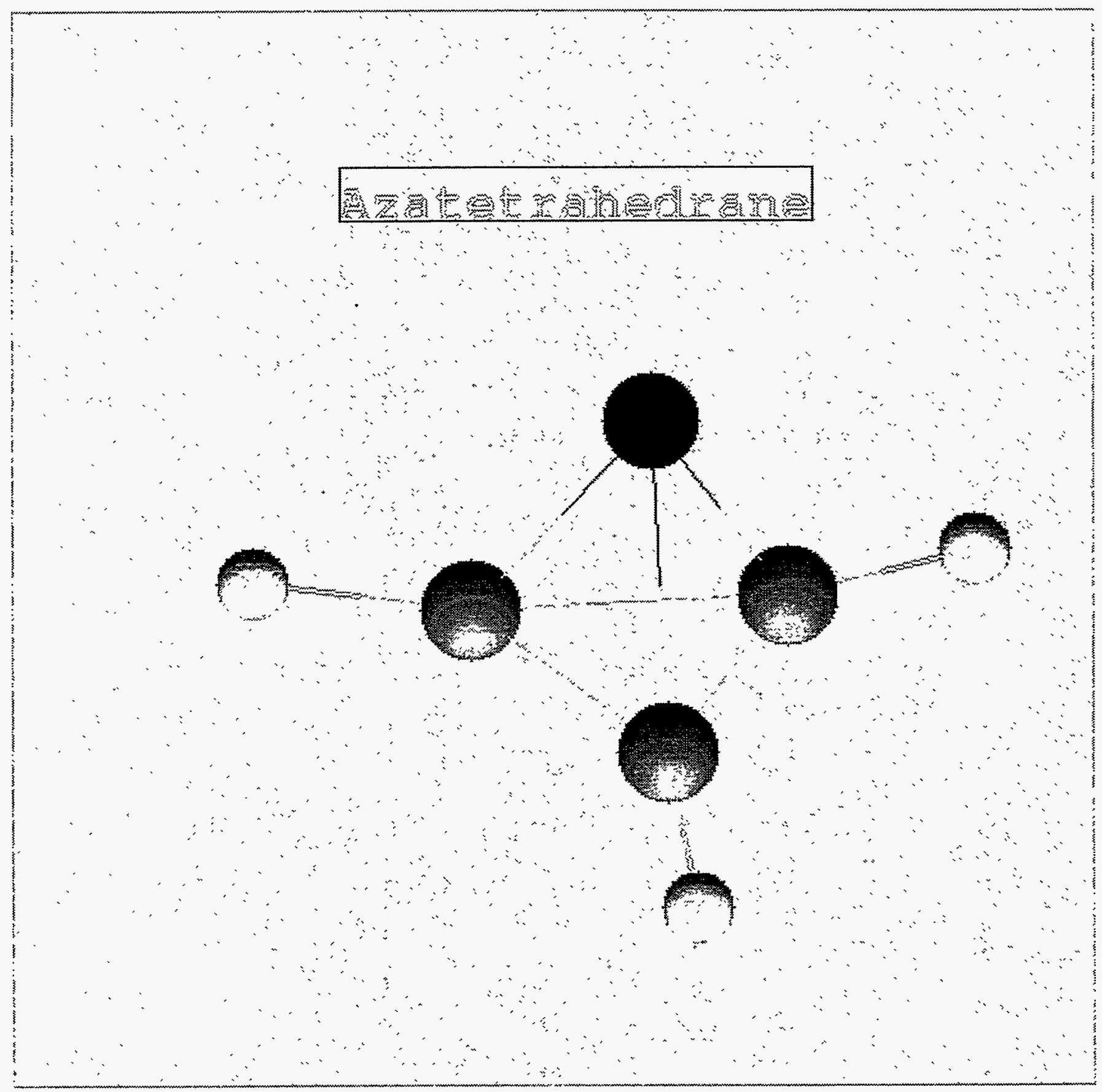

Figure 1-61 


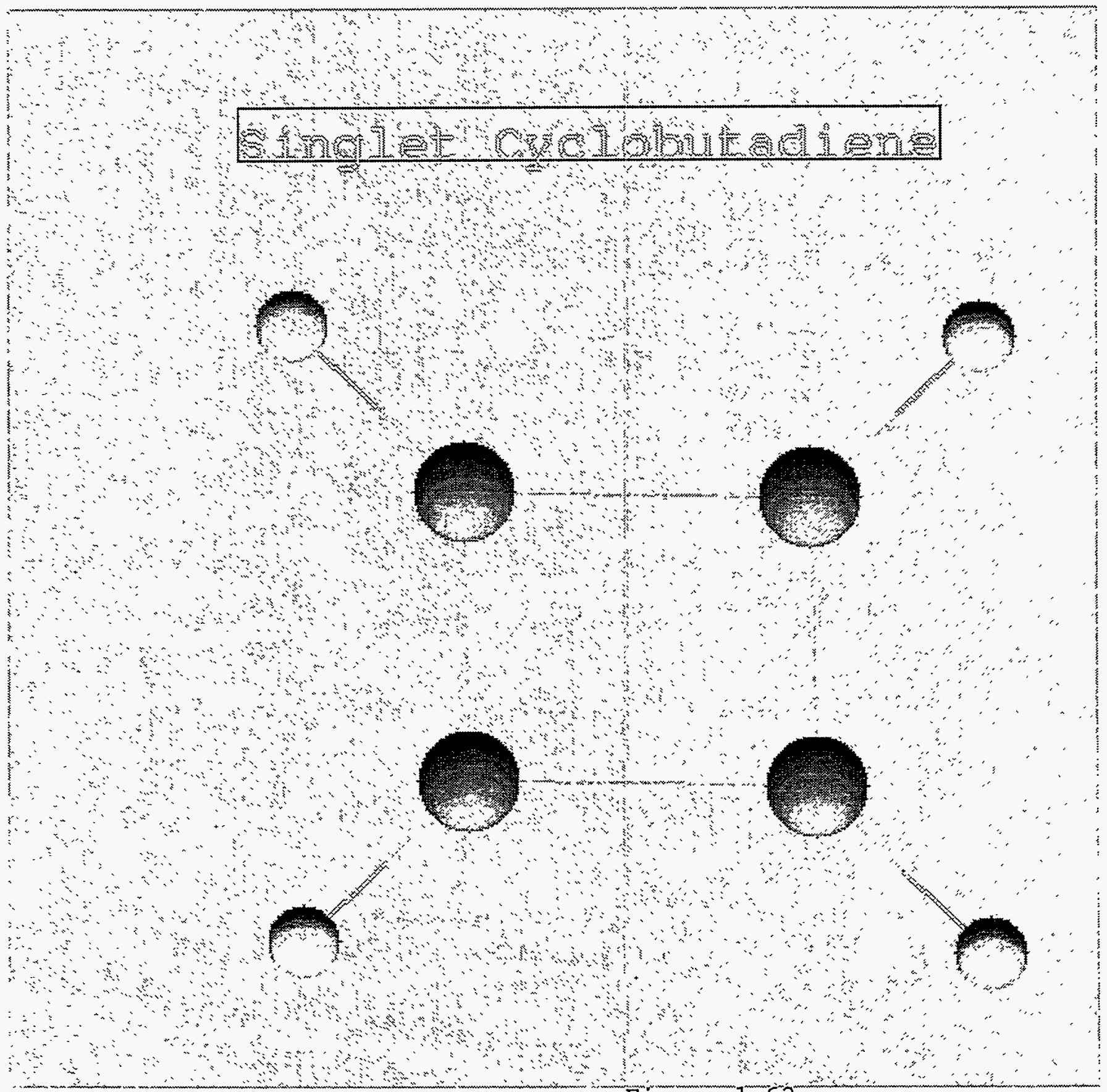




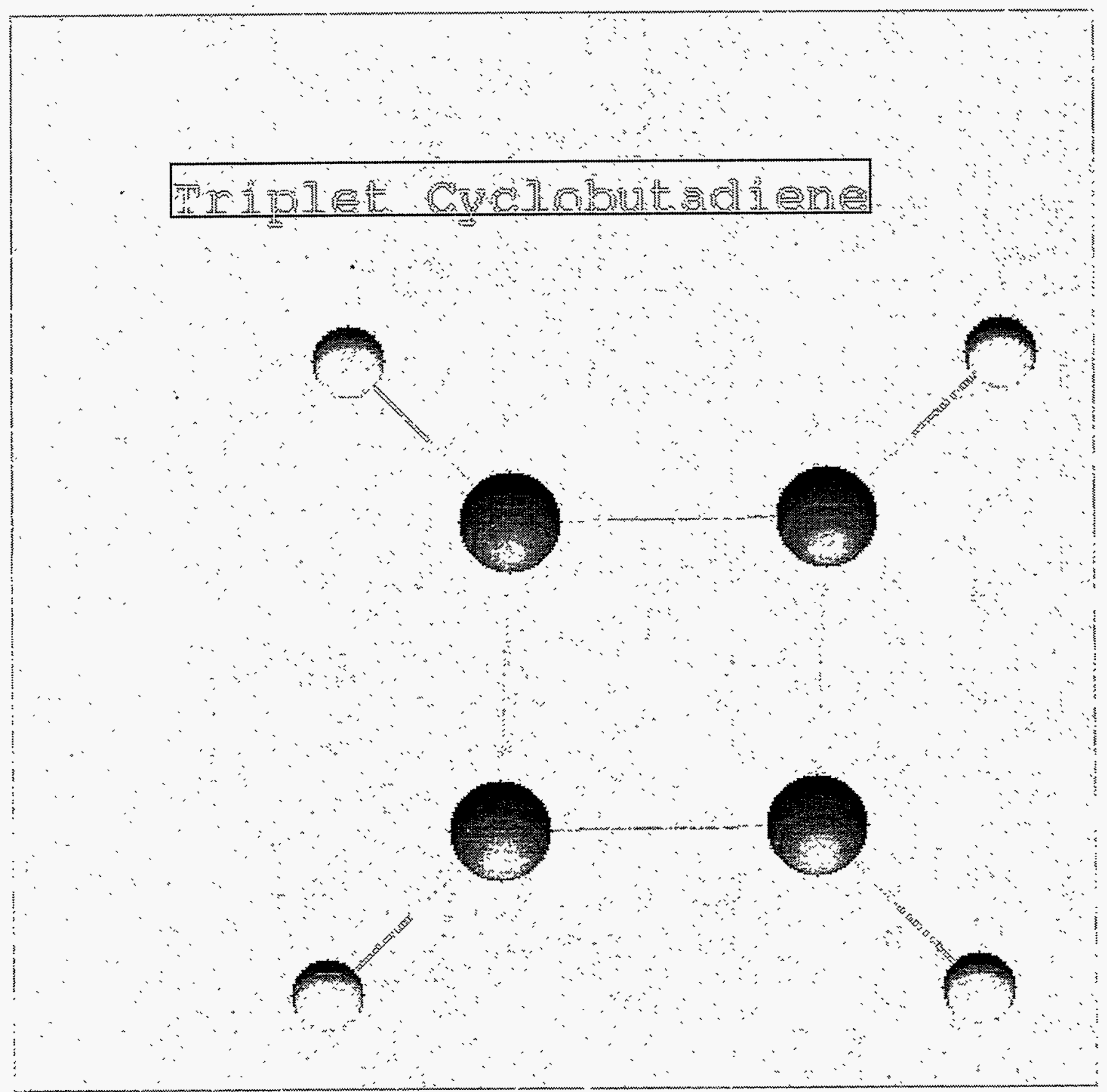

Figure 1-63 


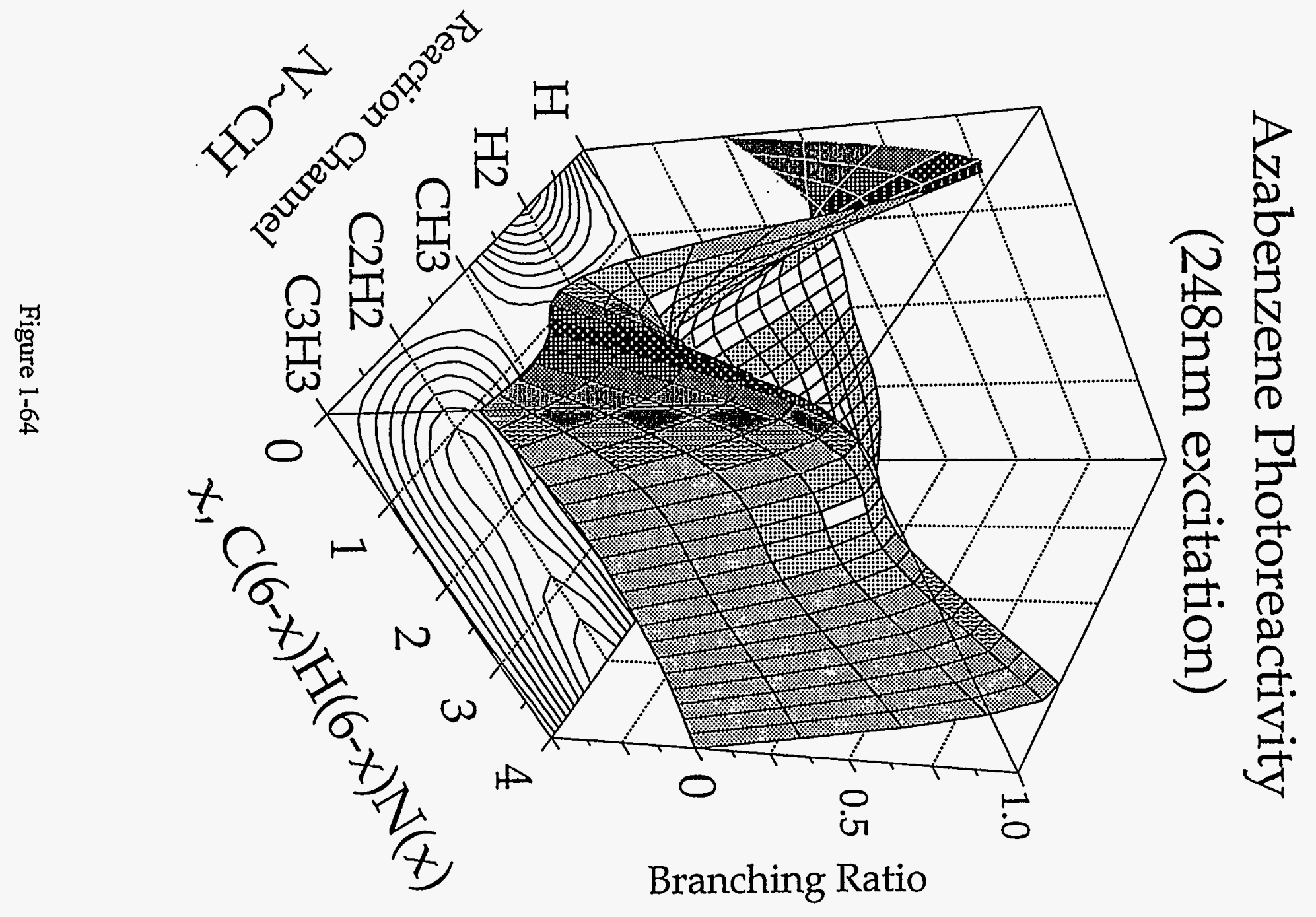




\section{Azabenzene Photoreactivity}

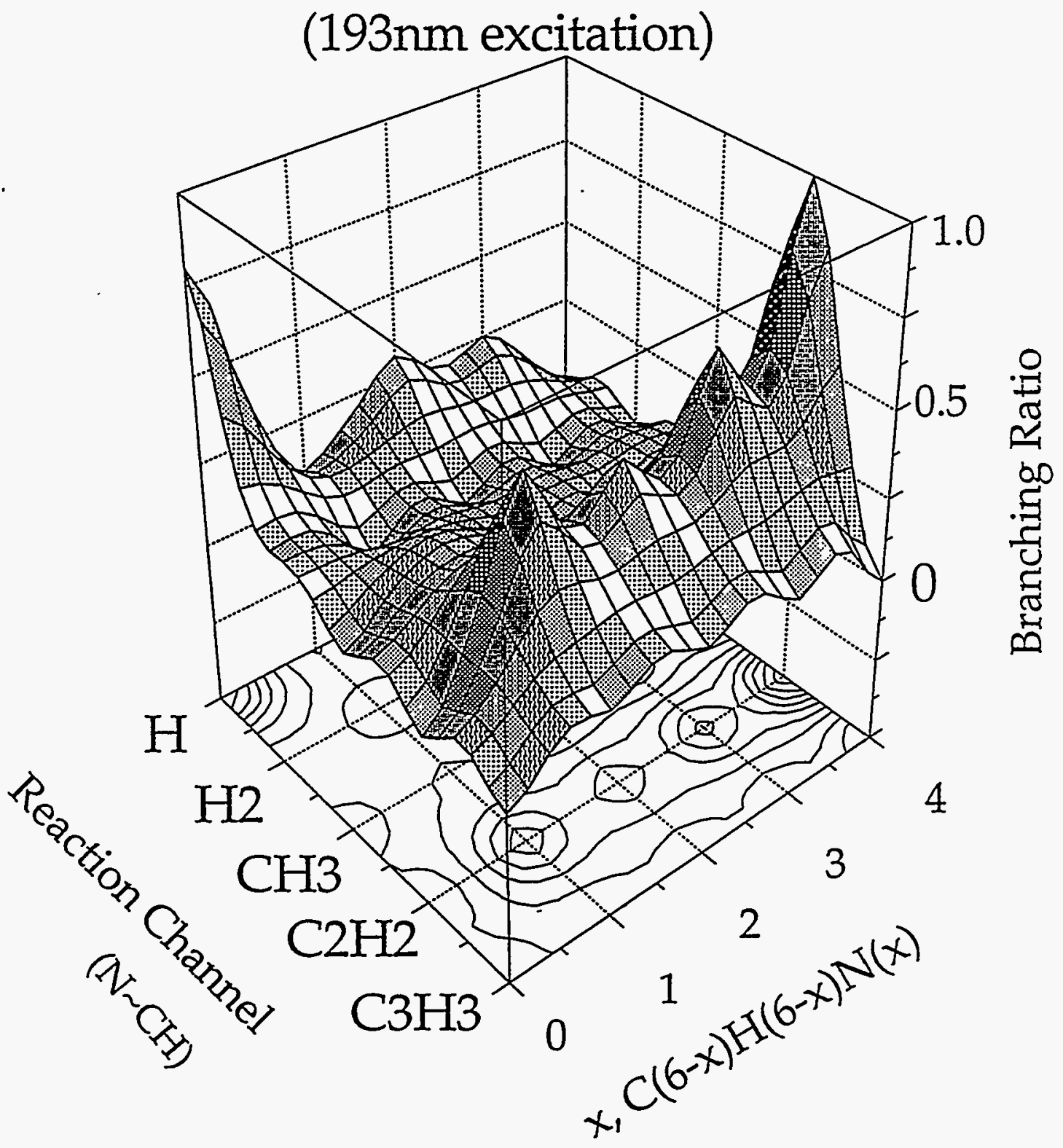

Figure 1-65 


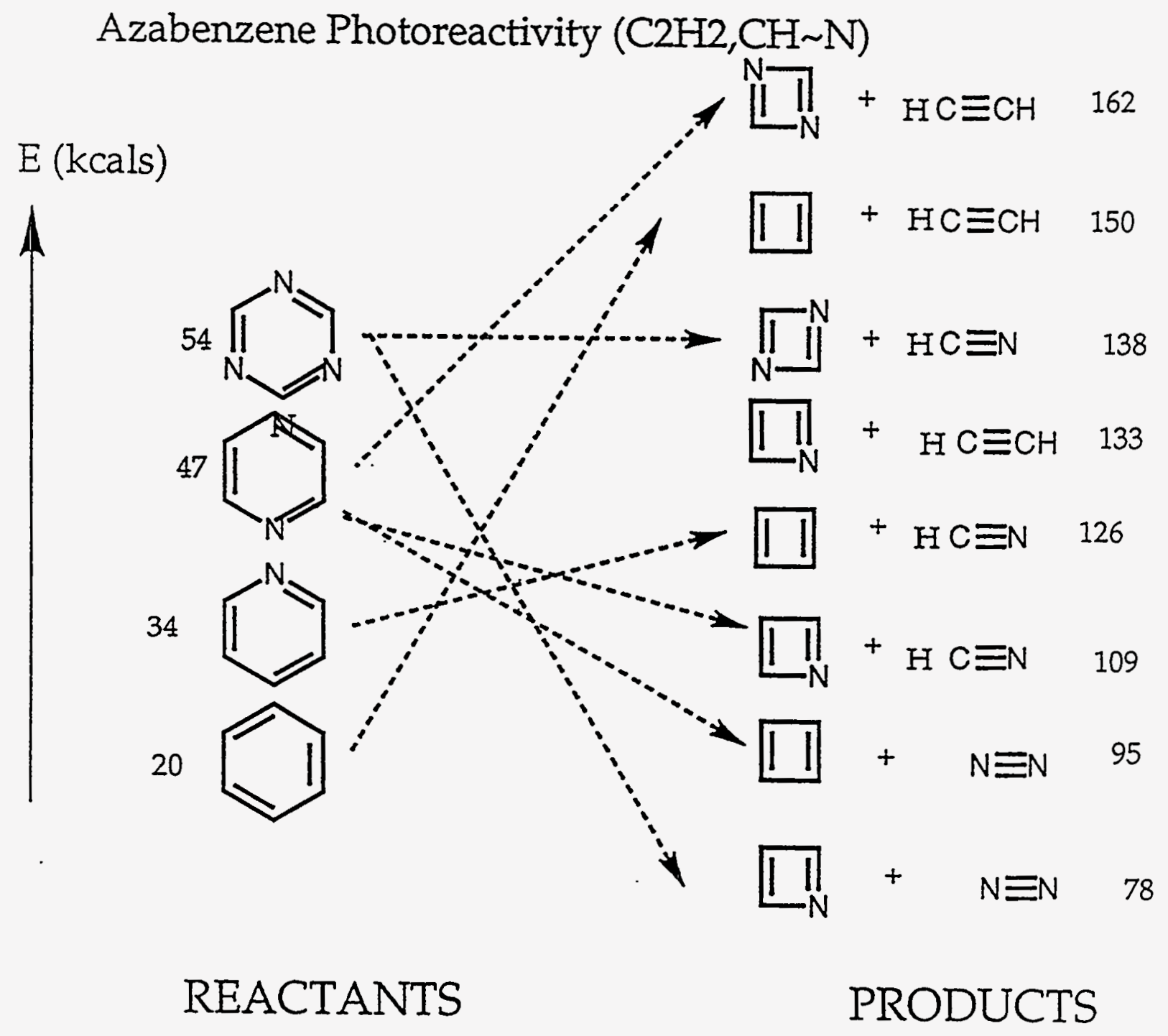

Figure 1-66 
Collisional Excitation of Translation, Rotation and Vibration

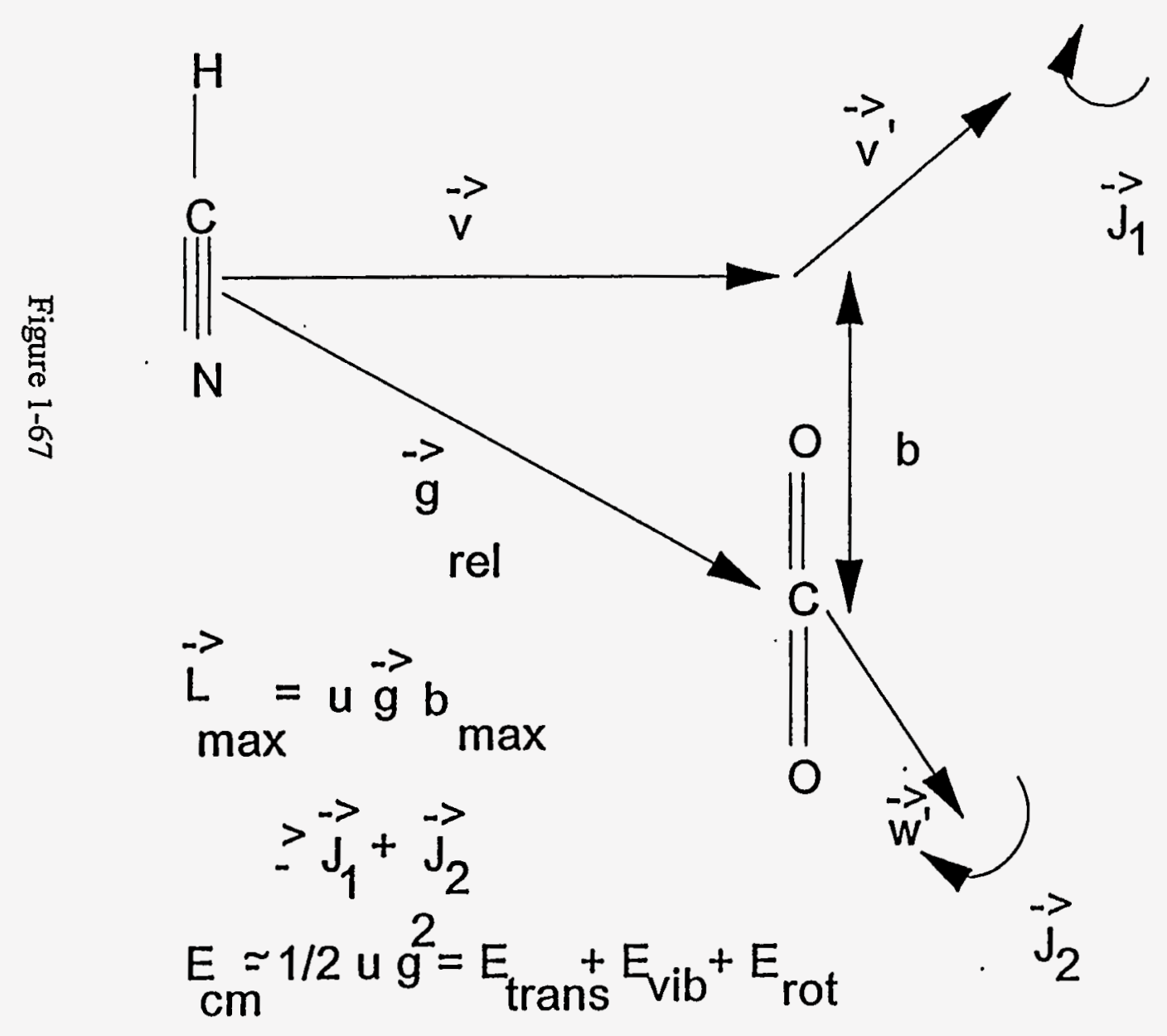



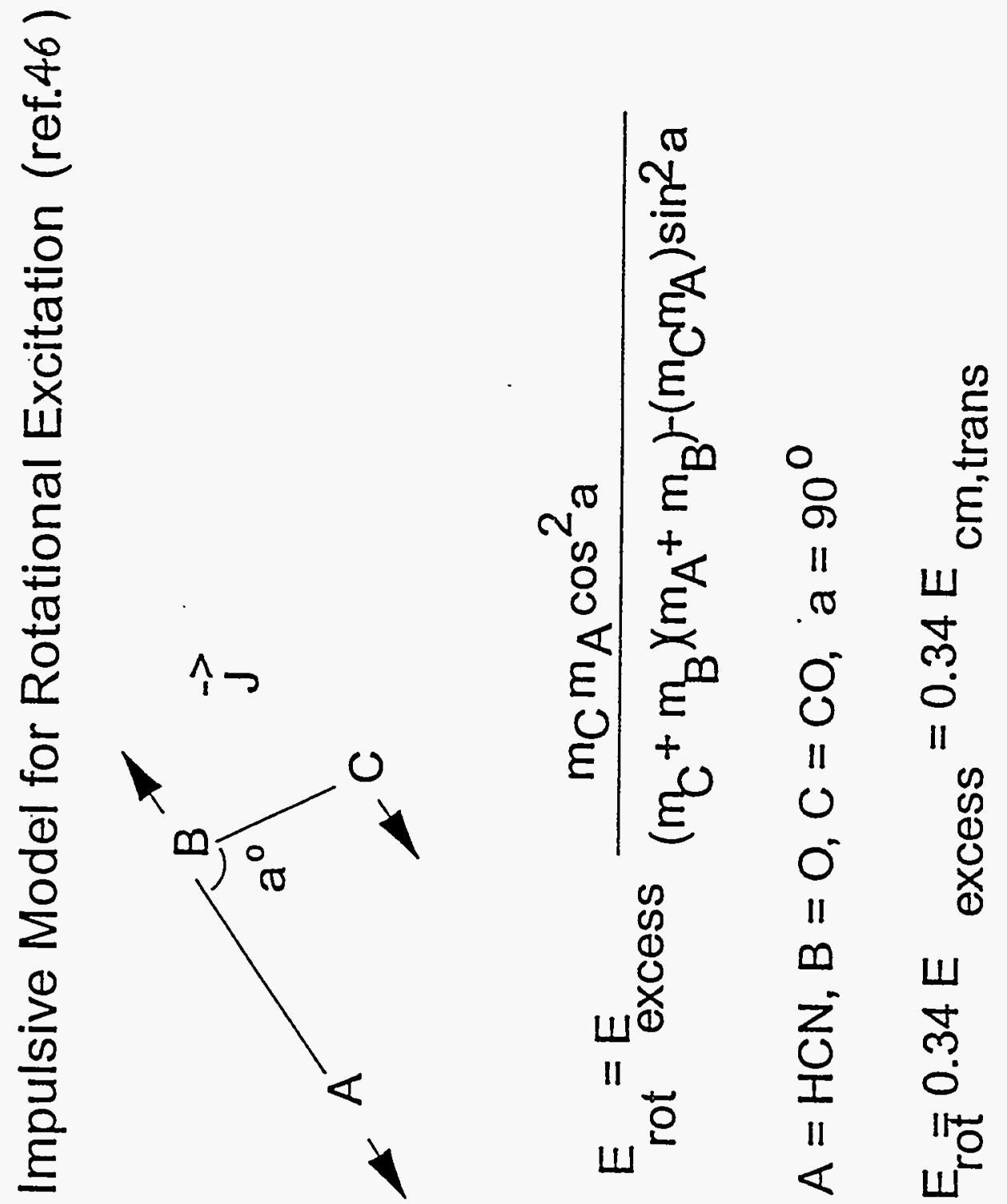

Figure 1-68 


\section{Symmetry, Spectroscopy and Dynamics}

A. Introduction

Solutions $\Psi$ to the Schrödinger equation

$$
\mathrm{H} \Psi=\mathrm{E} \Psi
$$

will possess symmetry properties of the Hamiltonian $\mathrm{H}$ as equation (1) will be invariant to the set of symmetry operations (and their inverses.) The simplifying role that application of this principle has had upon spectroscopic studies cannot be understated. Similarly, one can look at the time-dependent Schr ödinger equation

$$
i \hbar \partial \Psi / \partial t=H \Psi
$$

and its solution (the propagator equation)

$$
\Psi(t)=\Psi(0) \exp \{-i \mathrm{H} t / \hbar\}
$$

and recognize the same implications. The wave function, $\Psi(\mathrm{t})$, must evolve following a path which preserves the symmetry of the Hamiltonian in its exponential expansion (2). Knowledge of the Hamiltonian (and the system) is generally not detailed enough to allow rigorous application of this constraint throughout a physical transformation, and as a result one often speaks of the importance of 'symmetry breaking' in the course of a chemical reaction, in which the reactants separates into products, conserving only fundamental quantities such as (mass)-energy, linear and angular momentum. The relaxation process leading to a reaction stand on middle ground joining the spectroscopically well-characterized excitation to the asymptotic formation of products, 
and it is this path that we shall seek guidance across. In fairness we shall use the same rules and arguments that define how we arrived at our excited state of departure.

The probability of going from state $\mid i>$ to $\mid \mathrm{f}>$ mediated by an interaction $\mathrm{H}$ op is the square of the transition moment

$$
\mathrm{M}(\mathrm{f} \leftarrow \mathrm{i})=\int_{-\infty}^{\infty} f(x) \hat{H}_{o p} i(x) d \tau
$$

from which we can invoke the concept of a symmetry propagator

$$
\Gamma_{f} \otimes \Gamma_{o p} \otimes \Gamma_{i} \supset \Omega
$$

where $\Gamma_{\mathrm{j}}$ are the irreducible representations for which the (wave)functions $\mathrm{j}(\mathrm{x})$ and interaction $\mathrm{H}_{\mathrm{op}}$ form a basis for, and $\Omega$ is the totally symmetric irreducible representation of the point group of the system. The treatment is motivated strongly by spectroscopic transition theory, involving the interaction of matter with a radiation field via explicit mechanisms such as dipolar couplings (electronic transitions), polarizability (Raman transitions), infra-red active bands, vibronic origins. It finds a natural extension to relaxation processes in general, be they fluorescence and phosphorescence, radiationless transitions including internal conversion, inter-system crossing, intramolecular vibrational energy redistribution, or chemical reactions. The initial and final states are positions in phase space which connect the fluctuation (perturbation acting on the initial state) with the dissipation (final state or configuration), as table 1 details. In terms of statistical mechanics, these transitions will serve to decrease the value of the generalized Boltzmann $\mathrm{H}$ (ref. 42): 


$$
\overline{\bar{H}}=\sum_{k} P_{k} \log \frac{P_{k}}{G_{k}} .
$$

where $\mathrm{P}_{\mathrm{k}}$ is the probability of finding the system in a specified condition $\mathrm{k}$ corresponding to a group of $G_{k}$ unperturbed states. The time rate of change of this quantity will tend to a minimum (equilibrium condition) due to transitions into configurations (groups of states) which maximize the system's time dependent entropy, as the second law of thermodynamics and a definition of (the configuration dependent) $\mathrm{H}$

$$
H_{k}=-\log G_{k}
$$

require. One may imagine a spectroscopically well-defined transition as creating a perfected defined single quantum state (i.e. a zero entropy configuration) which will tend to redistribute its energy into various degrees of freedom through transit along the path of $\left\{G_{k}\right\}$ intermediate states which will re-establish a minima in $\mathrm{H}$.

Consideration of the details of vibronic excitation and relaxation suggests propensity rules concerning which electronic states and vibrational modes will be important in guiding the path of irreversible descent. For the case of absorption the transition moment can usually be separated into an electronic factor and a vibrational factor (neglecting rotations for the time being):

$$
R_{\text {everv }}=R_{\text {eer }} R_{v_{v *}}
$$

where

$$
\mathrm{R}_{\mathrm{v}, v^{*}}=\int \psi^{\prime}{ }_{\mathrm{v}}{ }^{*} \Psi^{\prime \prime}{ }_{v} \mathrm{~d} \tau_{\mathrm{v}}
$$

is referred to as the vibrational overlap, or Franck-Condon factor. Since the electronic transition is so rapid the nuclei can be thought of as being 'frozen' in a configuration 
(near equilibrium) and the excitation will vertically create an upper (excited) state at the geometry of the lower state. If the minima of the upper state is displaced along any coordinate the excited state will start oscillating about its minima, with the oscillatory motion created by a superposition of normal modes (vibrations) which are excited and form progressions (bands) in the spectrum. Since the ground vibrational state is symmetric the symmetry propagator (or selection rule) enforces that only symmetric excited vibrational states can be reached. For relatively similar excited and ground state geometries, the Franck-Condon factors will largely determine the intensity distribution of absorption lines. The $\Delta v_{k}=0$ (i.e. no change in any vibrational quantum number) will be very intense for any allowed transition (referred to as the $0-0$ band) since the ratio of intensity of the $0-0$ band to the sum of all intensities $v_{i-0}$ is (ref. 1 )

$$
\frac{I 0-0}{\sum_{u} I v_{i-0}}=\frac{\sqrt{\omega_{i} \omega_{i}^{\prime \prime}}}{1 / 2\left(\omega_{i}^{\prime}+\omega_{i}^{\prime \prime}\right)}
$$

For an excited state frequency half the ground state value $\left(\omega^{\prime}=1 / 2 \omega^{\prime \prime}\right)$ this ratio is still 0.943, emphasizing the importance of the Franck-Condon factor. The prominence of $\Delta v_{k} \neq 0$ transitions in absorption and emission spectrum is expected to be small although exceptional cases can arise, for example, when a formally forbidden transition becomes allowed by vibronic coupling. Such is the case for benzene, where the lowest electronic band $\left({ }^{1} B_{2 u} \leftarrow{ }^{1} A_{1 g}\right)$ is orbitally forbidden but allowed through the $e_{2 g}$ ring vibration $v_{18}$. In the case of pyrazine, the large transition strength of ${ }^{1} B_{3 u} \leftarrow{ }^{1} A_{1 g}$ is attributed to vibronic coupling via the $\mathrm{b}_{1 \mathrm{~g}}$ out-of-plane hydrogen bend $v_{10 \mathrm{a}}$ which can borrow 
intensity from the ${ }^{1} B_{2 u}$ state and thus appear in the fluorescence and absorption spectra. A second strongly coupled vibronic origin is the symmetric ring stretch $v_{6 a}$ which can account for an Raman intensity enhancement of $10^{6}$ (ref. 2) for pyrazine.

The critical property of these modes is their propensity to build sequences with $\Delta v_{k} \neq 0$ (i.e. changes in vibrational quanta) that have appreciable intensity to effect radiationless vibronic transitions. Energy conservation dictates that a radiationless transition from electronic state $\mid i>$ to $\mid f>$ satisfy:

$$
E_{f}-E_{i}-\sum_{i} n_{i, j} h v_{j}=-\sum_{f} n_{j, f} h v_{j}
$$

where $E_{k}$ is the electronic origin of state $k$ and $n_{1}$ the number of quanta in the mode with frequency $v_{1}$. For a given transition the modes(i) with $\Delta v_{\mathrm{i}}=0$ will have a Franck-

Condon factor independent of the number of vibrational quanta $v$, whereas the $\Delta v_{\mathrm{k}} \neq 0$ contributions will show strong dependence on $v$. For example, for an antisymmetric vibration with $\left|v_{k}^{\prime}-v_{k}{ }^{\prime \prime}\right|$ not large the $\Delta v_{k}=2$ vibrational overlap increases as $\left(v_{k}+1\right)$ meaning an intensity increase of $\left(v_{k}{ }^{\prime \prime}+1\right)^{2}$ in the +2 sequence (ref. 3). Heller et al. have determined a single vibrational mode overlap integral (ref. 44) between $\mathrm{m}$ and $\mathrm{n}$ quanta in the $\mathrm{a}^{\text {th }}$ mode to be

$$
1<m_{a}\left|n_{a}>\right|^{2}=\exp \left(-X_{a}\right)\left(m_{a} ! / n_{a} !\right) X^{n_{a}-m_{a}}\left[L_{m_{a}}^{n_{a}-m_{a}}\left(X_{a}\right)\right]^{2}
$$

where $L_{m_{a}}^{n_{a}-m_{a}}$ are generalized Laguerre polynomials. The rapid decrease in overlap with increasing vibrational change is summarized in table 1. 
Table 1. Calculated Franck-Condon Intensities for the triplet state of benzene (from reference 45).

\begin{tabular}{l|lll}
\hline Ground state & Out-of-plane bend & Carbon stretch & Hydrogen stretch \\
vibrational quanta & & & \\
\hline 0 & $7.63 \times 10^{-1}$ & 1.00 & 1.00 \\
1 & $1.91 \times 10^{-1}$ & $9.99 \times 10^{-4}$ & $8.91 \times 10^{-3}$ \\
2 & $3.78 \times 10^{-2}$ & $9.98 \times 10^{-7}$ & $7.96 \times 10^{-5}$ \\
3 & $7.48 \times 10^{-3}$ & $9.97 \times 10^{-10}$ & $7.09 \times 10^{-7}$ \\
4 & $1.49 \times 10^{-3}$ & $9.96 \times 10^{-13}$ & $6.33 \times 10^{-9}$ \\
5 & $2.95 \times 10^{-4}$ & $9.95 \times 10^{-16}$ & $5.03 \times 10^{-11}$ \\
6 & $5.84 \times 10^{-5}$ & $9.94 \times 10^{-19}$ & $4.49 \times 10^{-13}$ \\
7 & $1.16 \times 10^{-6}$ & $9.93 \times 10^{-22}$ & $4.01 \times 10^{-15}$ \\
8 & $2.30 \times 10^{-7}$ & $9.92 \times 10^{-25}$ & $3.57 \times 10^{-17}$ \\
9 & $4.56 \times 10^{-8}$ & $9.91 \times 10^{-28}$ & $3.19 \times 10^{-19}$ \\
\hline
\end{tabular}

To accurately model radiationless transition rates for benzene Burland et al. (ref. 45) must include a vibronic coupling mechanism which gives rise to anharmonicities and enhanced Franck-Condon overlaps for large changes in vibrational quanta. Coming from an equilibrium vibrational distribution the Boltzman weighting $\left(\exp \left[-\mathrm{G}\left(v_{\mathrm{k}}{ }^{\prime \prime}\right) / \mathrm{kT}\right]\right)$ will limit the observed intensity of such transitions; however, mechanisms which redistribute vibrational quanta into such 'promoting modes' may profoundly influence the 
chain of relaxation processes. An example of such a path is the near unit efficiency of singlet-triplet conversion in pyrazine, where a vibronically active mode $\left(v_{10 a}\right)$ which provides oscillator strength to the excited singlet state (i.e. perturbs the state) also acts as a promoting mode to intersystem crossing. Heller et al. have theoretically reproduced the strong energy dependence on radiationless rates by considering contributions from a small number of vibronically active modes in benzene (ref. 46). The experimentally observed predominance of intersystem crossing over internal conversion in aromatic systems (ref. 43 ) is rationalized by the energy gap dependence of the Franck-Condon factors.

The ability of vibrational energy to remain localized in certain modes can also influence relaxation pathways. The 'local mode' picture of $\mathrm{C}-\mathrm{H}$ stretch overtones has been used to explain the anomalous increase in fluorescence lifetime observed in deuterated aromatics such as benzene and napthalene (the 'deuteron effect'). By modelling the effect on excess energy on the fluorescence yields Lim et al. have shown that certain accepting modes may retain a larger portion of vibrational energy than their statistical partition (ref. 35) and thus influence the relaxation dynamics of the system. Although Franck-Condon factors will weaken such transitions in the excitation spectrum, the non-radiative relaxation path will be guided by their strength and character, as Kasha's rule suggests (ref. 46). One may imagine how such modes could also effect isomerizations and even chemical reactions in certain circumstances.

In summary, the perturbations one may observe in fluorescence and phosphorescence decay (vibronic origins, progressions, Fermi resonances) may be indicative of the prominent sequences present in the radiationless path. Such modes owe 
their presence to strong interactions between nearby surfaces that may cross, vibrations that may mix, displacements that induce geometric changes and even chemical reactions. Manifestations of the symmetry propagator (eq. 5) are implicit in reaction path treatments conserving orbital symmetry (Woodward and Hoffman rules) and will be described in a similar manner. The quantum mechanical propagator $\exp \{-i \mathrm{H} t / \hbar\}$ is often treated parametrically as a reaction coordinate. It characterizes the mapping of evolving (time dependent) states to their quasi-stationary destinations, or the flow of energy through the system with time. Through the lens of symmetry, a general description of radiationless transitions, the phenomena of resonant energy transfer, the Energy Gap Law, non-Born-Oppenheimer dynamics and unimolecular reaction rate theory can be enriched, as the photodissociation of 1,4 , Diazine may demonstrate. 
Table 2. Some important mechanisms for energetic relaxation in chemical systems

\begin{tabular}{|c|c|c|c|}
\hline Process & Final State & Initial State & Interaction \\
\hline Photon absorbtion & Excited singlet & (Ground) singlet & $\begin{array}{l}\text { Dipole-Field }(\mu . \mathrm{E}) \\
\mu \leftrightarrow \mathrm{x}, \mathrm{y}, \mathrm{z}\end{array}$ \\
\hline $\begin{array}{l}\text { Stimulated emission } \\
\text { Fluorescence }\end{array}$ & (Ground) singlet & Excited singlet & $\begin{array}{l}\text { Dipole-Field ( } \mu . E) \\
\mu \leftrightarrow \mathrm{x}, \mathrm{y}, \mathrm{z}\end{array}$ \\
\hline Raman scattering & $\begin{array}{l}\text { Vibrationally } \\
\text { excited }\end{array}$ & Vibrationally cold & $\begin{array}{l}\text { Polarizability }(\alpha) \\
\alpha \leftrightarrow \mathrm{x}^{2}, \mathrm{y}^{2}, \mathrm{z}^{2}, \mathrm{xy}, \mathrm{xz}, \mathrm{yz}\end{array}$ \\
\hline $\begin{array}{l}\text { Intersystem crossing } \\
\text { Phosphorescence }\end{array}$ & Triplet & Singlet & $\begin{array}{l}\text { Spin-Orbit Coupling } \\
\mathrm{H}_{\mathrm{so}} \leftrightarrow \mathrm{R}_{\mathrm{x}}, \mathrm{R}_{\mathrm{y}}, \mathrm{R}_{\mathrm{z}}\end{array}$ \\
\hline Internal conversion & (Lower) singlet & Upper singlet & $\begin{array}{l}\text { Vibronic Coupling, } \\
\text { Curve crossings }\end{array}$ \\
\hline Chemical reaction & Transition state & Ground state & $\begin{array}{l}\text { IVR (ergodic?), } \\
\text { curve crossings }\end{array}$ \\
\hline Chemical Structure & Conformers,Isomers & $\begin{array}{l}\text { Equilibrium } \\
\text { structure }\end{array}$ & zero point energy \\
\hline
\end{tabular}




\section{B. Orbitals, Symmetry and Photoreactivity}

The Schrodinger equation

$$
\left(\frac{-\hbar^{2}}{2 m} \nabla^{2}+V\right) \psi=E \psi
$$

is only soluble for a two particle system; these solutions are often referred to as hydrogenic wave functions and may be designated by a series of variables: the principal quantum number $n$, the azimuthal quantum number $l, m_{l}$ which describes the oriental of the orbital angular momentum vector $l$, and the spin $s$. To construct molecular orbitals which will ultimately be responsible for bonding and reactive properties a procedure which involves taking linear combinations of atomic orbitals (hydrogenic wave functions) is employed. This procedure implicitly generates symmetry properties in the resulting molecular orbitals (MOs) and provides a systematic framework for description and reference.

If we consider the effect of bringing two ground state hydrogen atoms (designated $\mathrm{A}$ and $\mathrm{B}$, with each electron in $\{n=1, l=0\} \leftrightarrow 1$ s orbitals) towards each other, the . anisotropy in the potential can give rise to symmetric and antisymmetric combinations

$$
\begin{aligned}
& \sigma_{\mathrm{g}}(1 \mathrm{~s})=1 / \sqrt{2}\left(\sigma 1 \mathrm{~s}_{\mathrm{A}}+\sigma 1 \mathrm{~s}_{\mathrm{B}}\right) \\
& \sigma_{\mathrm{u}}(1 \mathrm{~s})=1 / \sqrt{2}\left(\sigma 1 \mathrm{~s}_{\mathrm{A}}-\sigma 1 \mathrm{~s}_{\mathrm{B}}\right)
\end{aligned}
$$

where $\sigma$ is shorthand for $\left|\mathrm{m}_{\mathrm{l}}\right|=0$ and the subscripts $g$ (gerade, even) and $u$ (ungerade, odd) index properties of these new wavefunctions with respect to the molecule's center of symmetry. Through such a procedure we can rationalize the formation of molecular 
hydrogen $\left(\mathrm{H}_{2}\right)$, visualize the electron distribution in its the bonding $\left(\sigma_{\mathrm{g}}\right)$ and antibonding $\left(\sigma_{u}\right)$ orbitals and infer physical properties such as geometry and energetics. By bonding we note that the total energy of the system $E$ decreases as we move the atoms closer in from far away (an attractive interaction); by antibonding just the opposite (a repulsive interaction). Other atomic orbitals may be combined in a similar manner to form $\pi \quad\left(\left|\mathrm{m}_{\mathrm{l}}\right|=1\right), \delta\left(\left|\mathrm{m}_{\mathrm{l}}\right|=2\right)$ molecular orbitals which will have designations such as $2 \mathrm{~s} \sigma_{\mathrm{g}}, 3 \mathrm{p} \pi, 5 \mathrm{~d} \delta$. Once the electrons are distributed in a manner consistent with rules such as the Pauli exclusion principle, properties such as the total angular momentum

$$
\Lambda=\sum_{i} \lambda_{i}
$$

where the vector sum is taken over all $i$ electrons, each with angular momentum $\lambda_{i}$ and the total spin

$$
S=\sum_{i} s i
$$

which defines the multiplicity of the state $(2 S+1)$ can be derived.

The process of combining atomic orbitals usually is done in a pairwise manner, with the higher energy (valence) electrons forming the $\sigma$ bonding framework of the molecule. To satisfy the valence demands and achieve an electron configuration of a noble gas (a filled octet for group I and II atoms excluding hydrogen) additional electrons may need to be promoted or hybridized to form bonding orbitals. This step may lead to energetically similar structures which are called resonance forms. Many atomic orbitals may need to be combined to form a single molecular orbital to reflect an extended 
through space interaction or delocalization in the system. A prime example of such a system is the benzene molecule, and the phenomenon of aromaticity which arises from taking orthogonal combinations of six carbon p orbitals to form six $\pi$ orbitals (three bonding and three antibonding) and consequently filling them to achieve an energetic stabilization on the order of $37 \mathrm{kcal} / \mathrm{mole}$.

The chemical properties of a molecule are related to the symmetry properties of the corresponding orbitals involved in reactive transformations. This principle was enunciated by Woodward and Hoffman in "The Conservation of Orbital Symmetry" (ref. 1): "processes will occur readily when there is congruence between orbital symmetry of reactants and products." From orbital symmetry considerations we will be able to decide if certain transformations are allowed or forbidden in a manner similar to how a spectroscopist would categorize electronic transitions. To take a simple example first, we will consider the elimination of HCN from Dewar-Pyrazine.

To first approximation we will consider the process to have the same essential symmetry as the elimination of acetylene from Dewar-benzene. Referring to figure 2-1 (taken from ref. 8), the important detail to observe is that the ground state orbitals of reactants correlate with the excited state orbitals of the products. The implies that the reaction is formally forbidden in the ground state and conversely allowed in the excited state. In traversing from the reactant configuration to the products a symmetry imposed barrier is a consequence of the intended correlations between the orbitals. The reaction is described as a $\left[{ }_{0}{ }_{s}+{ }_{0}\right.$ s $]$ cycloreversion and shares the same symmetry considerations as the reaction run in the other direction, a $\left[{ }_{\pi}{ }_{s}+{ }_{\pi} 2_{s}\right]$ cycloaddition. The designation refers 
to the type of bond involved (first subscript), the number of electrons, and finally the orientation of the reactants (second subscript, $s=$ suprafacial and $a=$ antarafacial). As Lionel Salem points out, (ref. 3), the inducing symmetry coordinate which will facilitate a crossing between the two highest occupied molecular orbitals (HOMOs) of the reactant ( $\pi$ bonding $b_{1 u}$ electrons) and product ( $\sigma$ bonding $b_{3 u}$ electrons) is

$$
b_{1 u} \otimes b_{3 u}=b_{2 g}
$$

which for C-C stretching motion involves a stretching of one diagonal of the rectangle with a compression of the other diagonal, implying a transoid configuration with significant biradical character which is supported by theoretical calculations (ref. 4). In the photolytic pathway the lowest unoccupied molecular orbitals (LUMO) must be considered, and it correlates with an $\mathrm{a}_{\mathrm{u}}$ displacement as is noted by Raghavachari et al. (ref. 5) and an allowed process (ref. 1). Experimental results supports this analysis: when cyclobutadiene in an argon matrix is irradiated a pair of acetylene molecules are liberated upon warming up the matrix; if the matrix is just heated up in the dark no acetylene is formed, but rather syn-tricyclo[4.2.0.0]octa-3,7-diene is produced following a thermally allowed dimerization pathway (ref. 6) .

In the case of pyrazine the monoazete (single nitrogen substituted cyclobutadiene) is expected to follow similar behavior, although several mechanisms should contribute to enhanced reactivity. $A b$ initio calculations performed by myself on dewar pyrazine show each half to be an elongated rectangle with a shortened $\mathrm{C}$ double bond $\mathrm{N}$ distance due to increased electron localization towards the electronegative atom. The somewhat weakened and elongated single bonds are more prone to distort and pucker, inducing the 
$a_{u}$ twisting motion that will liberate $\mathrm{HCN}$ and a second azete fragment. As predicted from the correlation diagram, an exit barrier exists that will impart significant translational energy into the recoiling fragments.

The prediction that a ground state forbidden reaction (i.e. a thermal process) is excited state allowed (i.e. a photochemical process) can be used to rationalize the formation and stability of the important valence isomers of benzene and pyrazine. The correlation diagram for the rearrangement to benzvalene and dewar benzene is shown in figure 2-2 (taken from ref. 7). Disagreement still exists as to whether the benzvalene ground state isomerization should involve consideration of the local symmetry (ref. .9) or more global symmetry $\left(\mathrm{C}_{2 \mathrm{v}}\right.$, ref.7). Inspection of the relevant orbitals reveals that excited state (photo)-isomerization is symmetry allowed in both cases and that ground state isomerization is symmetry forbidden for Dewar benzene. This prediction is borne out in experiment: these molecules are synthesized through UV irridiation and possess some thermally stability (see refs. 10,11). Even the less stable dewar pyridine has been photoproduced and found to have a half-life of 36 minutes at zero centigrade (ref. 12). A similar effort to produce dewar pyrazine could be attempted: irradiation of a low temperature solution followed immediately by ${ }^{1} \mathrm{H}$ or ${ }^{13} \mathrm{C}$ nuclear magnetic resonance. The more stable pyrazine and its isomers (diazafulvene, and other diazines) are the expected products. The ${ }^{1} \mathrm{H}$ NMR spectrum we have taken so far has confirmed the purity of the diazine and triazine samples and their inability thermally isomerize.

A notable alternative to the concerted pathway to benzvalene involves a biradical intermediate. Bryce-Smith and Gilbert find that fulvene is invariably produced along 
with benzvalene and suggest a common precursor, prefulvene, which consists of fused three membered and five membered rings (ref. 13). Oikawa et al. have calculated the lowest energy triplet path for the process and find a biradical of such geometry (ref. 14). Halevi (ref. 7) notes that this triplet biradical could be formed out of $S_{1}$ through a surface crossing to the $T_{z}\left(b_{1}\right)$ manifold, which shares the same space-symmetry $\left(B{ }_{1}\right)$. with the $T_{1}$ states of both benzene and benzvalene. Weddel et al. see adiabatic conversion of benvzvalene to benzene only on the triplet surface. (ref. 14b)

The details of such a process in the case of pyrazine can be described as follows. Internal conversion to $S_{1}\left({ }^{1} B_{3 u}\right)$ is followed by intersystem crossing primarily to the triplet manifold with great efficiency ( $\Phi_{\text {isc }}=1.0$ from $S_{1}$ excitation and $\Phi_{\text {isc }}=0.13$ from $S_{2}$ excitation, see ref. 15). Because the significant chemical reactivity observed out of the $\mathrm{S}_{2}$ state, this second value could be low because it is based upon phosphorescence yields compared to an expected relaxation rate to $S_{0}$. Although the lowest triplet $T_{1}$ is ${ }^{3} B_{3 u}$, consideration of the coupling strength of spin-orbit interactions shows that states of different character (n $\pi^{*}-\pi \pi^{*}$ versus $\pi \pi^{*}-\pi \pi^{*}$ ) to be about two orders of magnitude larger (ref. 16). Thus from the donor state ${ }^{1} B_{3 u}\left(n \pi^{*}\right)$ the dominant accepting states are expected to be ${ }^{3} \mathrm{~B}_{2 u}\left(\pi \pi^{*}\right)$ and ${ }^{3} \mathrm{~B}_{1 \mathrm{u}}\left(\pi \pi^{*}\right)$ if energetically accessible. The spin flip process is accomplished by coupling spin angular momentum of an electron with orbital angular momentum, i.e. a change of one unit of angular momentum due to an electron changing spin along one axis must be compensated for exactly by the appearance of orbital angular momentum of the same magnitude but in the opposite direction along this axis. Such a 
process is generally rare for light atoms but is accomplished with effectiveness in pyrazine due to promoting modes of symmetry which transform as the rotations $\left(R_{x}, R_{y}, R_{z}\right)$ which can be thought of as re-orienting the $p$ orbitals $(l=1)$ and thus access the final state of triplet spin multiplicity according to the symmetry propagator:

$$
b_{3 u} \otimes\left(\begin{array}{l}
b_{3 g} \\
b_{2 g} \\
b_{1 g}
\end{array}\right) \otimes \gamma_{f i n a l}=a_{g}
$$

For the ${ }^{3} B_{2 u}$ state a $b_{1 g}$ mode satisfies this equation and the hydrogen out-of-plane bend $v_{10 \mathrm{a}}$ is strongly implicated in this process due to its strong vibronic activity and reduction in frequency from the ground state $\left(\mathrm{S}_{0}, 918 \mathrm{~cm}^{-1}\right)$ to the first excited singlet $\left(383 \mathrm{~cm}^{-1}\right)$ (see refs. 17 and 18 ). For the lower ${ }^{3} \mathrm{~B}_{1 u}$ state $v_{5 \mathrm{a}}$ of $b_{2 g}$ symmetry shows a smaller reduction in frequency from $757 \mathrm{~cm}^{-1}\left(\mathrm{~S}_{0}\right)$ to $518 \mathrm{~cm}^{-1}\left(\mathrm{~S}_{1}\right)$ and forms a weaker progression. For the lowest triplet state, ${ }^{3} \mathrm{~B}_{3 \mathrm{u}}$, modes of $\mathrm{a}_{\mathrm{u}}$ symmetry such as $v_{16 \mathrm{a}}$ and $v_{17 a}$ appear with intensity in the $T_{1} \leftarrow S_{0}$ absorption (see ref. 19). The rate of intersystem crossing is inferred to be about $5 \times 10^{9} \mathrm{~s}^{-1}$ (ref.15) based upon the fluorescence decay, although bi-exponential behavior is observed. The fast component arises from resonance fluorescence decay while the slower component is thought to come from weak coupling of sparse triplet levels with the strongly radiative singlet. From the singlet-triplet mixed state the system a slow decay into the ground state (on the order of $1 \mu \mathrm{s}$, see ref. 20) follows mostly non-radiatively by intersystem crossing as the phosphorescence radiative lifetime is about 0.010 s (ref. 21). 
The wavelength dependence of the isomerization yield suggests that an energy barrier must be overcome for the process to occur. This is consistent with the diradical hypothesis, whose most energetically demanding step will be the formation of the diradical bicycle, be it a singlet or triplet. At energies above $34000 \mathrm{~cm}^{-1}$ the isomerization quantum yields of about 0.05 (ref. 22) imply that the process is competitive with other relaxation mechanisms, but not dominant. Due to rapid internal conversion processes which can occur to funnel the higher triplets and singlets down to $S_{0}, S_{1}$ and $T_{1}$, Kasha's rules (ref. 23) suggest that the relevant electronic states we need consider are the ground state, first excited singlet as well as triplet. Early $a b$ initio work by Sobolewski and Domcke (ref. 24) suggest an internal conversion funnel is accessed on transit to the prefulvenic form which rapidly converts $S_{2}$ to $S_{0}$ when energy in $S_{2}$ rises above the threshold marked by the onset of 'channel 3' behavior in benzene.

Plotting the reactive and radiative pathways followed in pyrazine versus excitation energy (see figure 2-3) would suggest that channel 3 in pyrazine is inextricably related to the chemical reaction processes. The inverse correlation between fluorescence and phosophorescence on one hand and the expression of chemical products on the other shows how the balance is tipped near the fulcrum of $34000 \mathrm{~cm}^{-1}$ excitation energy. Going to a deeper mechanistic level, our experimental results suggest that extensive competition and interconversion between the valence isomers benzvalene, prefulvene/fulvene and dewar benzene guide the relaxation pathway towards qualitatively different sets of reaction products. Figure 2-4 depicts the passage from excited pyrazine to configurations leading to the various groups of reaction products we observed. The 
dominant photochemical channel liberates translationally fast $\mathrm{HCN}$ that is consistent with a $\left[{ }_{0}{ }_{s}+{ }_{0} s\right]$ cycloreversion from Dewar pyrazine. The release of $\mathrm{C}_{2} \mathrm{H}_{2}$ requires a second photon (for certain at $248 \mathrm{~nm}$, maybe also at $193 \mathrm{~nm}$ ) that more likely reflects the energetic instability of the required Dewar pyrazine isomer (both nitrogens separated in one ring), which lies about $43 \mathrm{kcal}$ higher (at the RHF/6-311+G* level) than the form with the nitrogens separated on the edge of each four membered ring (about $70 \mathrm{kcals}$ above ground state pyrazine). The most notable (and contraversial) isomerization product is $\mathrm{N}_{2}$ which would require two successive benzvalene isomerizations, has a dewar precursor much higher in energy (about $115 \mathrm{kcals}$ at $\mathrm{RHF} / 6-311+\mathrm{G}^{*}$ ), and shows a relatively low yield compared to the former two channels (about an order of magnitude less the acetylene). The characteristic 'double' velocity distributions observed are roughly consistent with the energy splittings calculated between the two spin states (triplet and singlet) of the azete products, which at the MP2/6-311+G* level predicts about a $1 \mathrm{eV}$ difference with the triplet lying slightly higher for the monoazete and diazete. The possibility of two parallel processes contributing from separate singlet $\left(S_{0} / S_{1}\right)$ and triplet $\left(T_{n}\right)$ manifolds is suggested. The assignment of the faster peak to the singlet pathway is assisted by intensity dependent studies which show that this peak will disappear first as the excitation probability is raised in a manner similar to how the signal peaked near zero translational energy can be nulled (see figure 1-25). Greater excitation will mean greater excess energy and lower survivability of the $\mathrm{m} / \mathrm{e}=53$ fragment as suggested by the $193 \mathrm{~nm}$ spectra. There is also a possibility of a multiphoton contribution, given the relative yield into the slower peak. 
The work of Sobelewski (ref. 24) also finds the triplet pathway to be slightly lower in energy. Experimental studies of triplet sensitized isomerization of the diazines (ref. 31) find donors of high triplet energy $\left(\mathrm{Hg} 6{ }^{3} \mathrm{P}_{1} \sim 105 \mathrm{kcal}\right)$ to be effective sensitizers while lower energy donors $\left(\mathrm{Cd}^{3} \mathrm{P}_{1} \sim 88 \mathrm{kcal}\right)$ to be inefficient. The implication of a high triplet state in the isomerization process is thus quite strong.

Properties of the triplet hypersurfaces are discussed by Michl (ref. 36). Greater freedom of motion and looser geometries are more favorable because there is no longer a need for an ordered, cyclic array of orbitals such as in the excited singlet. In the case of cyclobutene, the $T_{1}$ surface leads downhill to a butadiene geometry, and even further downhill to a twisted butadiene. The case of cyclobutene mirrors the local symmetry of half the dewar isomer. From an excited state, the a $u$ twisting motion serves to promote the $\left[2{ }^{2}+2 s\right]$ cycloreversion that will liberate $H C N$ plus an azete. Modes such as $v_{16 a}$ and $v_{17 a}$ are of the correct symmetry and show a presence in fluorescence spectra, coupled via Fermi resonances to the relatively strong $v_{6 a}$ ring distortion that contains displacements which are similar to the geometry change between the $S_{0}$ and $T_{1}$ states.

More recent work by Stock and Domcke (ref.25) attribute ultrafast $\mathrm{S}_{1} \leftarrow \mathrm{S}_{2}$ dynamics to strong vibronic coupling as the dominant relaxation from the $\mathrm{S}_{2}$ state. The largest diabatic coupling element is for the $v_{10 a}$ mode and scales linearly with the normal coordinate $\mathrm{Q}_{10 \mathrm{a}}$; it is the activity of this mode that we attribute the propensity for intersystem crossing $\left(T_{3} \leftarrow S_{1}\right)$ to. 
Competing with the reaction processes on the $S_{1}$ and $T_{n}$ manifolds are transitions which convert the system to the ground state $S_{0}$. From the electronic relaxation studies (ref. 15) the radiative pathways (fluorescence, phosphorescence) are clearly much less efficient than radiationless transitions. The rate of transition between two states decreases very rapidly (often exponentially) as the energy gap between the electronic states grows (ref. 34) and the classic Landau-Zener expression for crossing probability is

$$
p=\exp \left[\frac{-\pi^{2} g^{2}}{h v \delta s}\right]
$$

where $g$ is the energy gap, $v$ the relative velocity at the crossing point, and $\delta$ s the difference in slopes at the avoided crossing. Transitions are thus facilitated at weakly avoided crossing points where the wavefunction can hop from one surface to the next, or at strongly avoided crossings between electronic levels where the wavefunction will pool at the minima of the higher state and undergo a vertical vibronic transition to near the saddle point of the strongly perturbing lower level below. Factors influencing radiationless transitions in nitrogen heterocycles have been investigated by Wassam et al. (ref. 35) and the predominant vibronic transitions associated with both internal conversion and intersystem crossing are described theoretically. For large energy gaps high frequency vibrations associated with hydrogen motion (e.g. stretches) are the dominant 'drains' or accepting modes, but for the case of diazines the out-of-plane bend $v_{10 \mathrm{a}}\left(\mathrm{b}_{1 \mathrm{~g}}\right)$ provides $80 \%$ of the transition moment strength while skeletal modes take less than $20 \%$ of the energy in the $S_{0} \leftarrow T_{1}$ relaxation. Our experimental results suggest that given enough excess energy in the $T_{1} / T_{n}$ state (following $S_{2}$ and even more so for $S_{3}$ 
excitation) conversion to the the $\mathrm{S}_{0}$ state occurs at a competitive rate with chemical reactions out of $S_{1} / T_{n}$. Figure 2-4 maps the atlas of reactive processes available to pyrazine while figure 2-5 delineates the expression of each channel as a function of excitation energy. From the two one can see how products channels associated with $\mathrm{S}_{0}$ reactions require excess energy to be competitive with products from the excited manifold. The internal conversion rate depends upon excess energy in a manner similar to the fluorescence decay rate observed in napthalene (ref. 35). The photochemical reaction product distribution is strongly dependent upon the excess energy in the excited electronic state as a function of the competing radiationless transition rates.

The slowest channels releasing $\mathrm{HCN} / \mathrm{C}_{3} \mathrm{H}_{3} \mathrm{~N}, \mathrm{~m} / \mathrm{e}=40$ (nitrogen substituted propargyl radical or azirine pairs) are thought to originate from the ground state. The internal conversion process could involve prefulvene or perhaps direct ring opening, as the strength of a C-N aromatic bond is expected to be about $87 \mathrm{kcal}$, about $13 \mathrm{kcals}$ lower than the $\mathrm{C}-\mathrm{C}$ aromatic bond according to the resonance energy treatment of Dewar and Trinajistic (ref. 26). For benzene, the ring opening to a linear form is more likely to favor the fulvene pathway and we believe this to be the case for pyrazine as well. The diaza-fulvene species can act as an intermediate either from either the singlet or triplet diradical which have energies comparable to pyrazine's $S_{1}$ and $T_{1}$ states. The thermochemistry of the $\mathrm{CH}_{2} \mathrm{CN}$ species is similar to the $\mathrm{C}_{3} \mathrm{H}_{3}$ (propargyl) radical but again emphasizes the stabilizing influence of the nitrogen substitution. While the propargyl radical has an estimated heat of formation of $81.5 \mathrm{kcal} / \mathrm{mol}$ (ref. 28), the nitrogen species is estimated to be $57.8 \mathrm{kcal} / \mathrm{mol}$, corrected by $10.8 \mathrm{kcal}$ of resonance 
stabilization by Benson (ref. 29). The origin of the recoil energy (about $5 \mathrm{kcals}$ at $248 \mathrm{~nm}$ ) can be attributed to electronic rearrangement necessary to effect this stabilization, while the additional channel at $193 \mathrm{~nm}$ could conceivable arise from production of the cyclic azirine radical, expected to be about $30 \mathrm{kcals}$ less stable by group additivity methods.

Ground state rearrangement have been suggested for pyrazine compounds from pyrolytic studies (ref. 27a) yielding mostly $\mathrm{HCN}$ and $\mathrm{C}_{2} \mathrm{H}_{2}$, but also small amounts of pyrimidine and pyridine. The latter product must result from recombination, although evidence of nitrogen migration comes from pyrolysis of 2,3-dicarboxylic anhydride yielding dicyanoethylene (ref. 27b). As is suggested by the ab initio studies, mass spectral data and synthetic efforts, the azetes are not stable enough to survive a pyrolytic oven. Thermal decomposition products of pyridine perhaps give a better clue as to primary steps which can be expected in pyrazine: at low temperatures cyanoacetylene and acetylene are the principal species liberated, although these products are likely from a chain reaction starting from $\mathrm{H}$ atom loss and the production of ortho-pyridyl radicals, which undergo ring opening to the stable cyano radical. At higher temperatures $\mathrm{HCN}$ elimination and the production of acrylonitrile is observed, which is a very likely candidate for the translationally slow products we observe in pyrazine. Hydrogen atom migration must be relatively facile for such a rearrangement to occur. The strongest evidence of $\mathrm{H}$ migration is provided for from brief studies done on pyrimidine $(1,3$ Diazine) which show $\mathrm{CH}_{3}$ elimination similar to that observed in benzene and pyridine (ref. 32,33). Since no detectable amount of methyl elimination is observed from 
pyrazine, the mechanism seems to require at least three contiguous $\mathrm{CH}$ moieties to allow the hydrogens to load up on the terminal carbon and then be eliminated as a methyl radical. The process involves a number of elementary steps: a minimum of one [1,2] shift and one $[1,3]$ shift, or may proceed by a series of three $[1,2]$ hydrogen migrations. A most plausible pathway would involve the fulvene as an intermediate, which is a low energy pathway to the open chain form and already has a terminal $\mathrm{CH}_{2}$ group. It also demonstrates that if the nitrogens do migrate it is generally towards the outer ends of the chain, as previous pyrolytic studies suggest.

The lost of translationally fast $\mathrm{H}$ atoms is consistent with an exit barrier from an antibonding (repulsive) coordinate. In the well known case of $\mathrm{H}$ loss from toluene (ref. 36) the interaction between the $\mathrm{C}-\mathrm{H}^{3} \pi \pi^{*}$ and ${ }^{3} \sigma \sigma^{*}$ orbitals, the latter of which correlates with $S_{0}$ products asymptotically, can induce a barrier to dissociation which can be thought of coming from rearrangement of the transition state to a separated $\mathrm{H}$ atom and pyrazyl radical, the latter of which is expected to show stability similar to the pyridyl analog; hence the relatively large contribution of this channel. Loss of 'slow' $\mathrm{H}$ (energy peaked near zero) from simple bond rupture in $\mathrm{S}_{0}$ also is seen following $193 \mathrm{~nm}$ excitation and more efficient conversion to the ground state.

Notable 'structure' could be resolved on the molecular deuterium elimination channel. The distinct $\mathrm{D}_{2}$ signals indicate a marked vibrational progression in the deuterium stretch. As mentioned in the previous chapter, a concerted mechanism liberating vibrationally excited molecular hydrogen from a transition state geometry displaced from equilibrium hydrogen-hydrogen separation is suggested. 


\section{Conclusion}

The primary photochemistry of pyrazine shows a remarkable progression and richness following ultraviolet excitation. The observed fall-off in radiative relaxation efficiency is counterbalanced by a dramatic increase in photochemical reaction yields and products. The product distribution into various channels suggests a complex interplay on the excited singlet and triplet surfaces between valences isomers and biradical intermediates which each serve to promote different processes. The dewar pyrazine geometry is conducive to a cycloreversion eliminating $\mathrm{HCN}$ and a monoazete; the benzvalene allows isomerization to both the pyrimidine and pyridazine forms; the fulvene facilitates passage to an open (linear) form which can extensively rearrange and eliminate both radical (methylene nitrile) and closed shell products. A 'pre-fulvene' [1,4] diradical may be a common precursor to the latter two isomers, and may favor a low energy triplet pathway. Triplet intermediates and products may be very pronounced in virtually all the channels, but especially in the case of the dominant azete plus hydrogen cyanide reaction, since low lying singlet and triplet states are predicted by ab initio calculations and two distinct product distributions are also observed.

The detailed passage through spectroscopically determined states was described with as much care as possible. Propensity rules for such transitions were considered on the basis of fundamental quantum mechanical properties such as spin multiplicity, energy and symmetry. The role of vibronic coupling to promote spin orbit interaction, internal conversion, motion along a reactive coordinate and other processes essential to the 
description of non-Born-Oppenheimer dynamics was manifested in the reaction path model. This model strongly implied the presence of excited state dynamics reaction based upon consideration of orbital symmetry and was provided a framework for understanding the energy dependence of the product distributions. The spectroscopically detailed understanding of pyrazine is thus woven into a more comprehensive picture of the elementary processes which act in competition and in parallel to guide the path of relaxation to a chemical reaction event. 


\section{References}

1. R.B. Woodward and R. Hoffman, "The Conservation of Orbital Symmetry," Verlag Chemie, New York, 1970.

2. Gerhard Herzberg, "Atomic Spectra and Atomic Structure," Dover, New York, 1944.

3. Lionel Salem, "Electrons in Chemical Reactions: First Principles," John Wiley and Sons, New York, 1982.

4. R. Hoffman, S. Swaminathan, B. Godell and R. Gleitner, J. Am. Chem. Soc., 92, 7091 (1970).

5. K. Raghavachari, R.C. Haddon and J.S. Binkley, Chem. Phys. Lett., 122, 219(1985).

6. O.L. Chapman, C.L. MacIntosh and J. Pacansky, J. Am. Chem. Soc., 95, 793(1973).

7. E. Halevi, "Orbital Symmetry and Reaction Mechanism," Springer Verlag, Berlin, 1992.

8. E.A. Halevi, Angew. Chem., 88, 664 (1976).

9. N.T. Anh, ed. note in Nouv. J. Chim., 1, 229(1977).

10. E.E. van Tamelen, S.P. Pappas and K.L. Kirk, J. Am. Chem. Soc., 93, 6092 (1971).

11. D. Bryce-Smith and A. Gilbert, "Rearrangements in Ground and Excited States," ed. de Mayo, Academic, 1980; Vol. 3, p. 349.

12. J.M. Coxon and B. Halton, "Organic Photochemistry," 2nd ed., Cambridge University Press, 1987.

13. D. Bryce-Smith and A. Gilbert, Tetrahedron 32, 1309 (1976).

14. S. Oikawa, M. Tsuda, Y. Okamura and T. Urabe, J. Am. Chem. Soc., 106, 6751 (1984). 
15. Iwao Yamazaki, Toshiro Murao, Takaya Yamanaka and Keitaro Yoshihara, Faraday Diss. Chem. Soc., 75, 395 (1983).

16. S. K. Lower and M.A. El-Sayed, Chem. Rev. 66, 199 (1966).

17. S. N. Thakur and K.K. Innes, J. Mol. Spectroscopy, 52, 130 (1974).

18. D. MacDonald and S.A. Rice, J. Chem. Phys., 74, 4893 (1981).

19. J.L. Tomer et al., J. Chem. Phys., 88(3), 1528 (1987).

20. Phaedon Avouris, William Gelbart and M.A. El-Sayed, Chem. Rev., 77(6), 793.

21. Hans Agnen, Olav Vahtras, Soren Knuts and Poul Jorgenson, Chemical Physics 181 (1994), 291-304.

22. W.A. Noyes, Jr. and K.E. Al Ani, Chem. Rev., 74(1974), 29.

23. G.N. Lewis and M. Kasha, J. Am. Chem. Soc., 66(1944), 2100.

24. A.L. Sobelewski and W. Domcke, Chemical Physics Letters, 180(4), 381.

25. Gerhard Stock and W. Domcke, J. Phys. Chem., 97(1993), 12466-12473.

26. M.J.S. Dewar and N. Trinajistic, Theoretica Chim. Acta, 17(1970), 235-238.

27. a) W.D. Crow and C. Wentrup, Tetrahedron Letters, 27, 3115 (1968).

b) R.F.C. Brown, W.D. Crow and R.K. Solly, Chemy Ind., 343(66).

28. C.H. Wu and R.D. Kern, J. Phys. Chem., 91, 6291.

29. H. Edward O'Neal and Sidney W. Benson, "Thermochemistry of Free Radicals," chapter 17 of "Radicals, Part III: Structure and Energetics," Wiley, 1977.

30. J.C. Mackie, M. Colket III and P.F. Nelson, J. Phys. Chem., 94(1990), 4099-4106.

31. F. Lahmani and N. Ivanoff, J. Phys. Chem., 76(16), 2245 (1972). 
32. A. Yokoyama, X. Zhao, E.J. Hintsa, R.E. Continetti and Y.T. Lee, J. Chem. Phys., 92(7), 4222.

33. K.A. Prather and Y.T. Lee, Israel Journal of Chemistry, 34, 43 (1994).

34. B.R. Henry and W. Siebrand, "Radiationless Transitions," chapter 4 in "Organic Photochemistry," ed. Bird, Wiley and Sons, 1976.

35. S.F. Fischer, A.L Stanford and E.C. Lim, J. Chem. Phys., 61(2), 582 (1974).

35. W.A. Wassain and E.C. Lim, J. Chem. Phys., 68(2), 433(1978).

36. J. Michl, Photochemistry, Vol. 46 of Topics in Current Chemistry, Springer Verlag, Berlin, 1974.

37. G.S. Ondrey and R. Bersohn, J. Chem. Phys., 81(10), 4517.

38. J.R. Fraser, L.H. Low, N.A. Weir, Can. J. Chem., 53, 1456-1460.

39. J.H. Glownia and S.J. Riley, Chem. Phys. Lett., 71(3), 429.

40. Zhao, X. Ph.D. Thesis, UC Berkeley, Nov. 1989, Ch. 3.

41 G.K. Vemupalli and T. Cassen, J. Chem. Phys., 56, 271.

42. R.C. Tolman, The Principles of Statistical Mechanics, Oxford University Press, 1950.

43. G.W. Robinson, in "Excited States," Vol. I, E.C $>$ Lim, ed. Academic Press, New York, 1974;

B.R. Henry and W. Siebrand, in "Organic Molecular Photophysics," J.B. Birks, ed., Wiley Interscience, London, 1973;

44. E.C. Lim, "Excess Energy Dependence of Vibrational Relaxation and Photophysical Branching Ratios in Isolated Aromatic Molecules: Relevance to Vacuum Ultraviolet Photochemistry", in Photophysics and Photochemistry in the Vacuum Ultraviolet, S.P. 
McGlynn, G.L. Findley and R.H. Huebner, Eds., D. Reidel Publishing Company, Dordrecht, 1985.

45. D.M. Burland and G.W. Robinson, J. Chem. Phys., 51(10), 4548 (1969).

46. D.F. Heller, K.F. Freed and W.H. Gelbart, J. Chem. Phys., 56(5), 2309 (1971). 


\section{Figure Captions}

Fig. 2-1. An orbital correlation diagram for the elimination of acetylene from cyclubutadiene. The $\left[2_{0}{ }_{s}{ }_{\sigma}\right]$ cycloreversion of $\mathrm{HCN}$ from dewar pyrazine is expected to share the same essential symmetry; the one set of $b 2 \mathrm{u} \pi$ electrons will not be present in the resulting azete fragment, but they correlate directly from products to reactants. Since the highest occupied molecular orbital (HOMO) of the reactant correlates with the lowest unoccupied molecular orbital (LUMO) of the product, the reaction is ground state forbidden and requires a perturbation of b2g symmetry to occur, as depicted by the arrow. The reaction is excited state allowed, formally requiring an au displacement (twist) which is likely an energy lowering distortion or large amplitude motion on the excited triplet and singlet surfaces.

Fig. 2-2. This orbital correlation diagram for the isomerization of benzene shows the important bonding (lower orbitals) and anti-bonding (highest orbital) involved in the transformation. The intended correlation of the highest occupied molecular orbital (HOMO) of benzene the antibonding (LUMO) of the dewar compound shows the reaction is ground state forbidden. Longitudinal excitation of an electron into the S2 state will make the reaction formally allowed. Note that only states of the same symmetry are subject to the non-crossing rule. In the case of benzvalene isomerization, electron promotion to the S1 state is the preferred (and allowed) pathway, although by adopting the global symmetry approach the reaction may be allowed from the ground state, but would certainly require significant activation energy. Not shown in the possible triplet 
pathway, which could involve a diradical intermediate, or in the case of pyrazine, transition orbitals of non-bonding character.

Figure 2-3.

A plot of radiative versus reaction quantum yields as a function of excitation energy for pyrazine. The phosphorescence yield is expressed as a ratio to the maximum intensity observed and thus represents the efficiency of intersystem crossing which then can radiatively relax to the ground state. The striking inverse correlation between chemical reaction probability and radiative relaxation efficiency demonstrates the strong energy demands required to open a reaction channel or other radiationless processes.

Figure 2-4

A plot of the important valence isomers and their role in determining the expression of different chemical reaction pathways. Although many of the forms such as the benzvalene and dewar pyrazine may interconvert through a relatively long lived pyrazine excited state, the fulvene may irreversibly relax to a linear form through ring opening, followed by rearrangements that may include ring closure to the highly vibrationally excited ground state. The benzvalene intermediate may share a common diradical precursor which bestows a strong energy dependence on the isomerization which can be effected through this valence isomer. The dewar benzene form leads to facile HCN elimination leaving a monoazete. The other isomers of the dewar form are higher in energy but can be accessed by two photon processes or one very energetic photon. 
Figure 2-5.

A diagram of the photodissociation pathways expressed by pyrazine. Products out of most channels were experimentally identified and are labelled accordingly. Secondary processes occurring after $\mathrm{H}$ atom loss or rearrangements following ring opening are more speculative. The role of valence isomers and proposed intermediates along different paths is emphasized.

Figure 2-6

A plot of channel yield versus excitation energy for pyrazine. The dramatic increase in chemical products following excitation energies over $34000 \mathrm{~cm}^{-1}$ marks the onset of fast radiationless processes leading to both isomerization and chemical reaction. At moderate energies reaction attributed to the excited state manifold dominate, whereas at higher energies other processes such as simple bond rupture ( $\mathrm{H}$ elimination ) and ring opening (via a high energy intermediate) are more strongly expressed in the product distribution. 
Correspondence Diagram for the Fragmentation of Cyclobutadiene
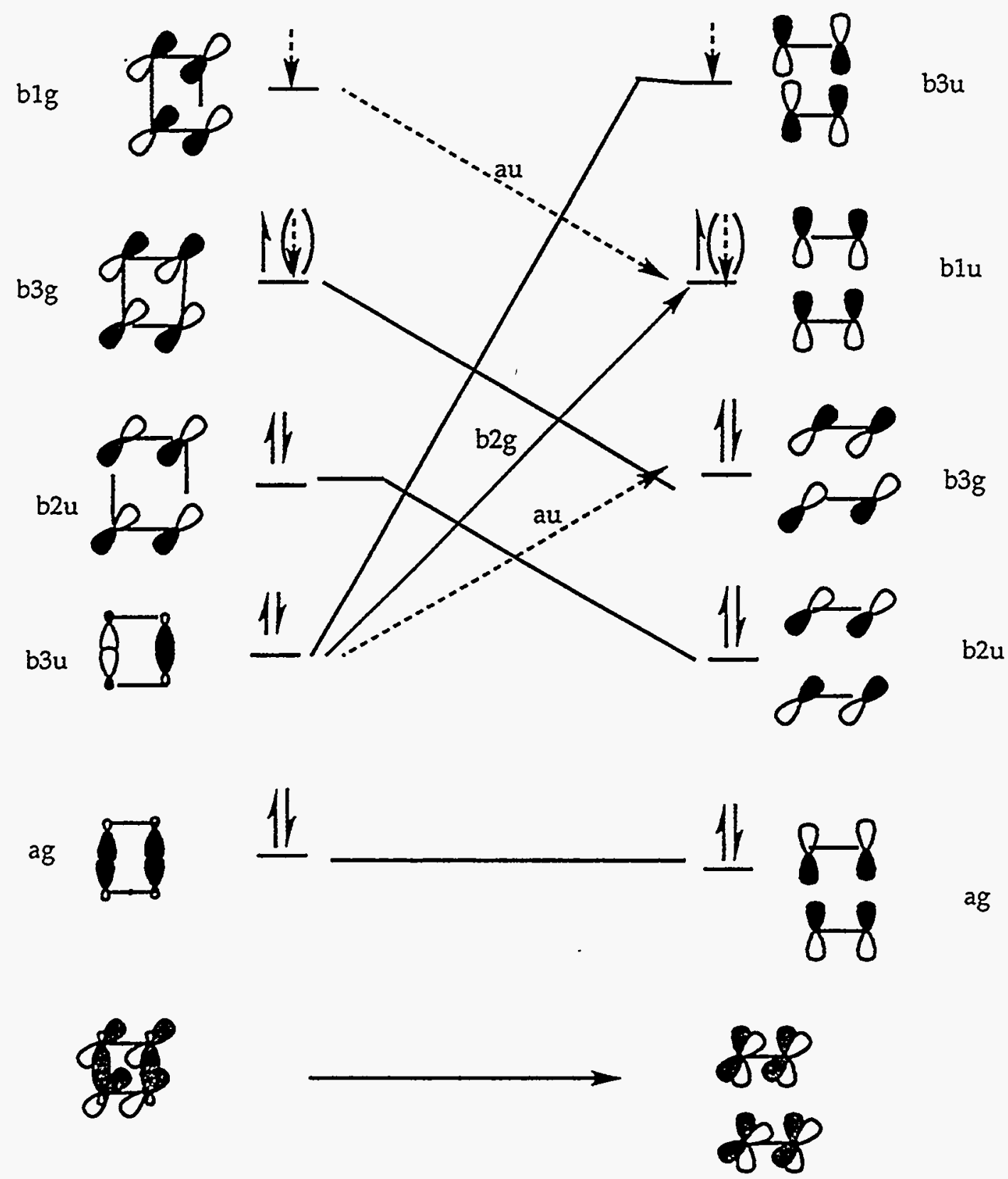

cyclobutadiene

2 acetylenes

The thermolytic pathway involves solid lines and arrows and is forbidden The photolytic pathway involved dashed lines and is allowed

Figure 2-1 
Photoisomerization of Benzene to Benzvalene and Dewar Benzene

Benzvalene (C2v(x))

Benzene (D6h)

Dewar Benzene (C2v(z))

$8 f^{8} 8-7$

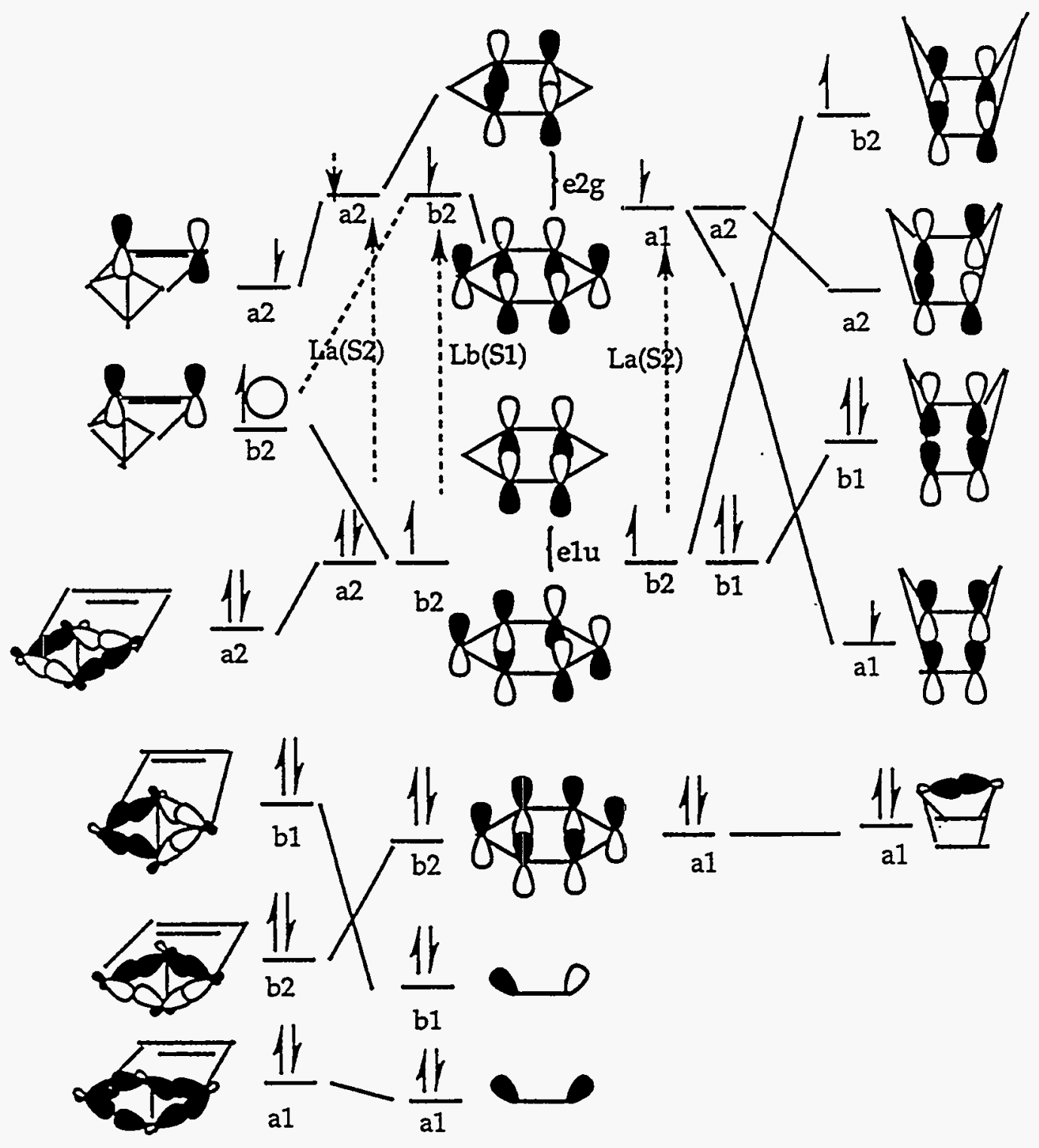

Figure 2-2 


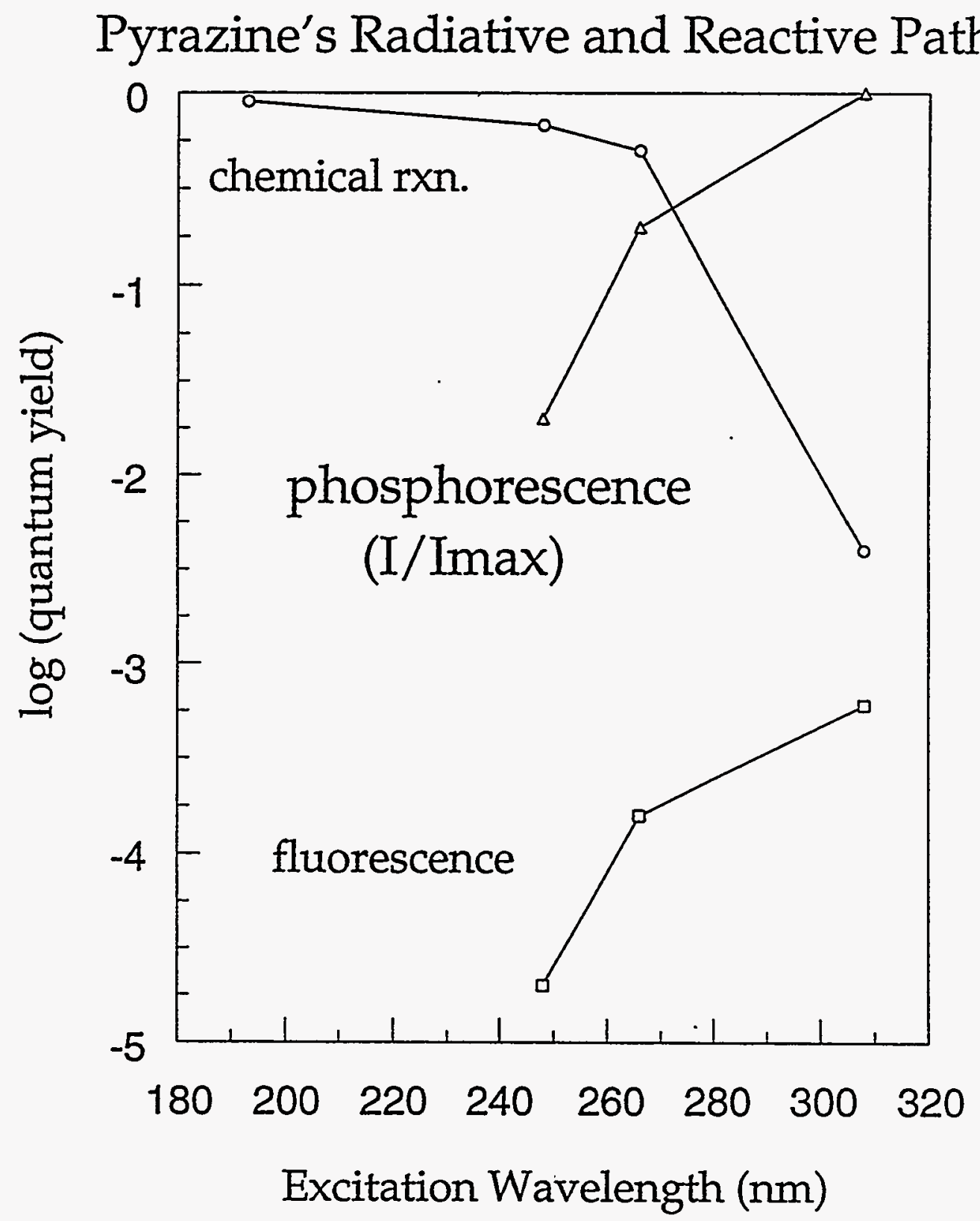

Figure 2-3 


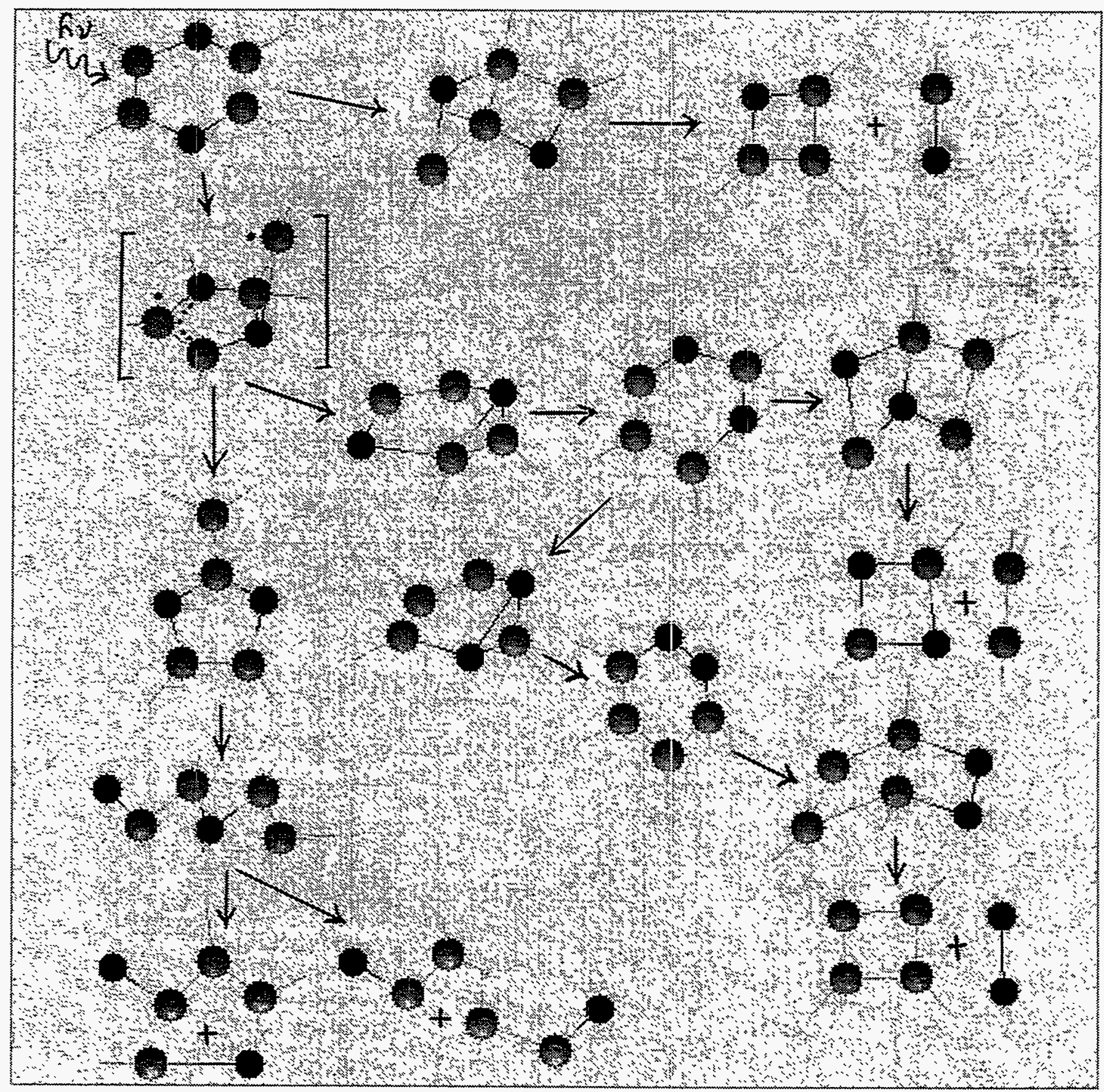

Figure 2-4 


\section{Pyrazine Photodissociation Pathway}

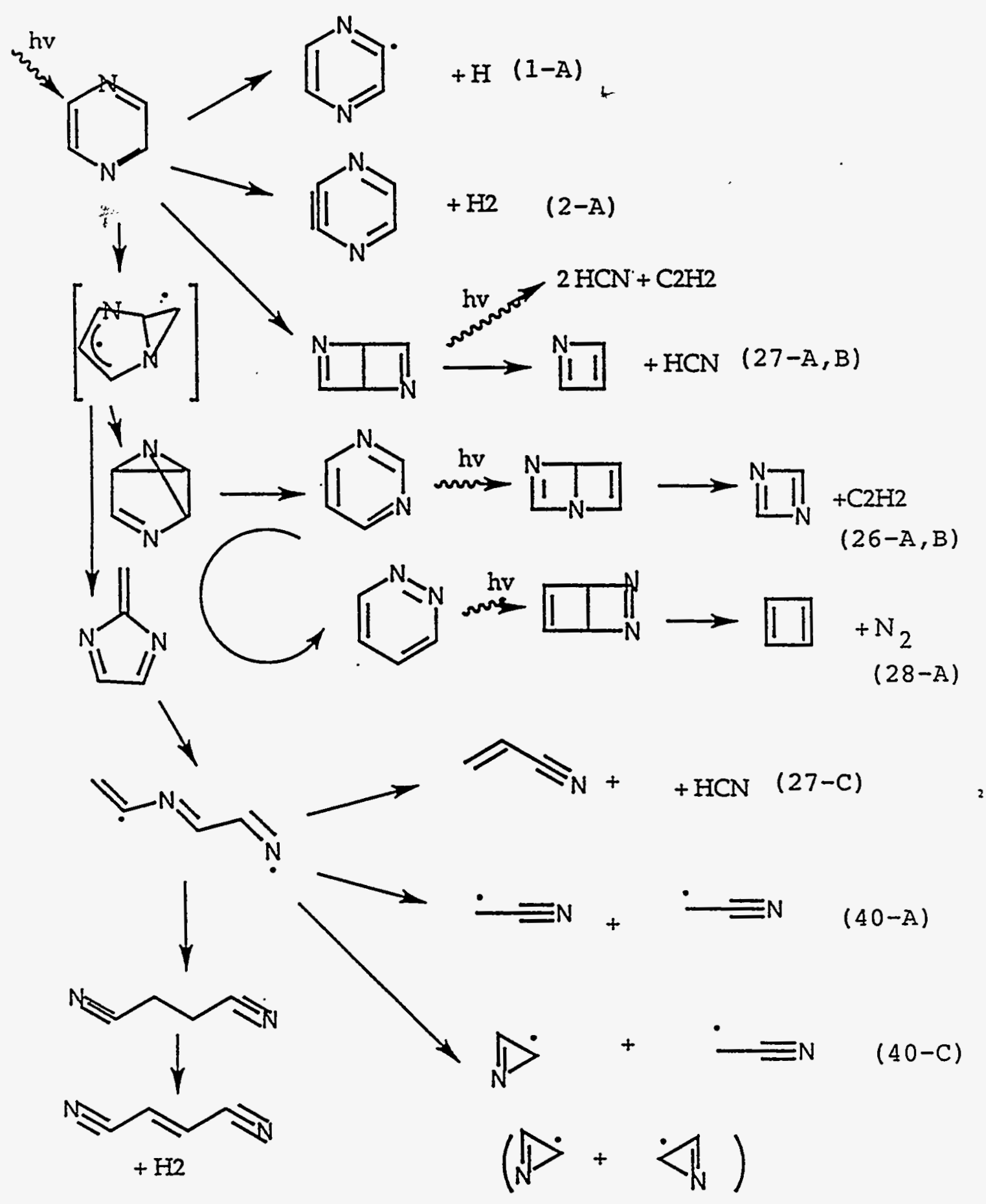

Figure 2-5 


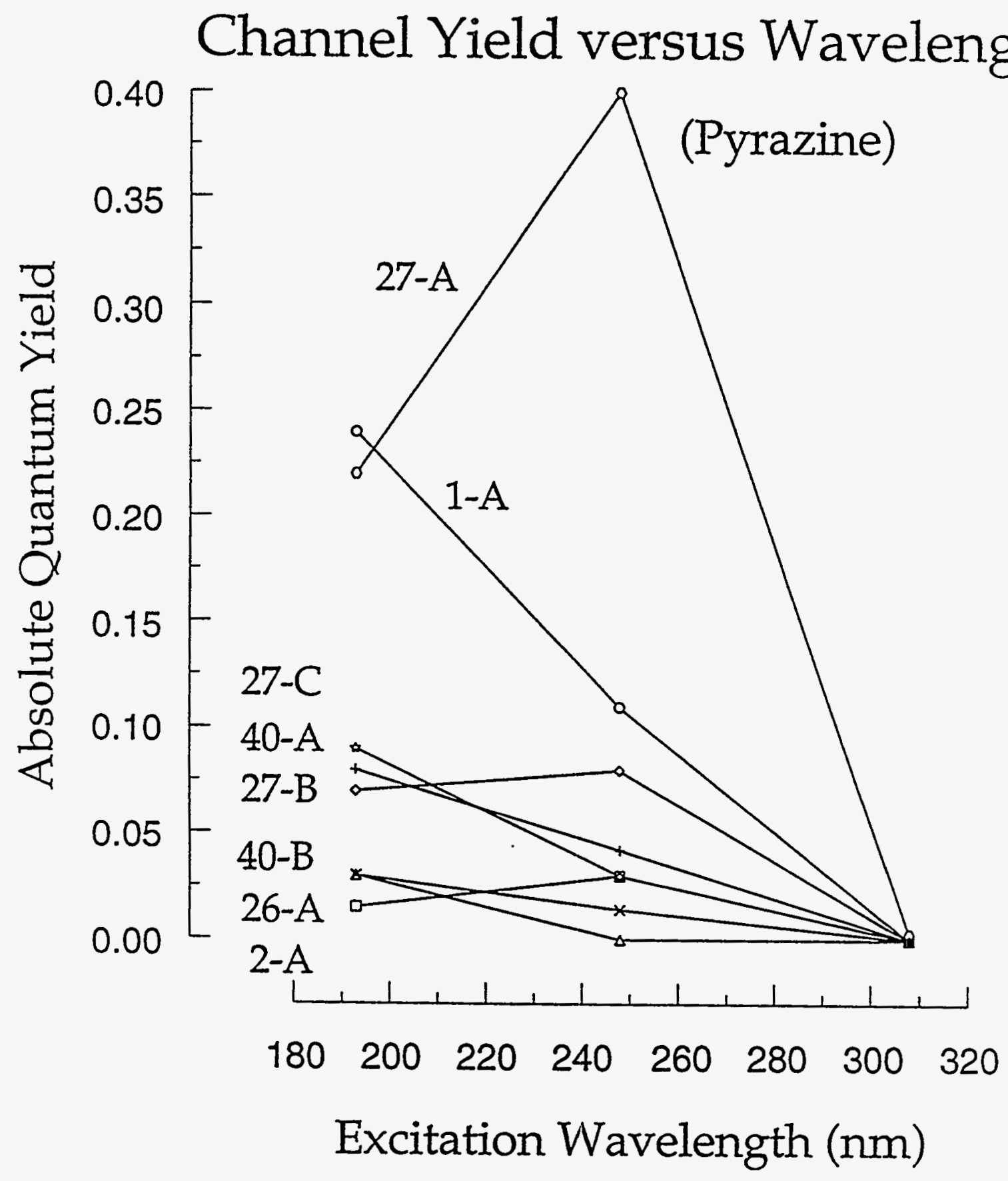

Figure 2-6 